Max-Planck-Institut

für ausländisches und

internationales Strafrecht

\title{
Forschungsbericht 2006/2007
}

Max-Planck-Institut für ausländisches und internationales Strafrecht 


\section{FORSCHUNGSPROGRAMM UND FORSCHUNGSPROJEKTE}
A. Zielsetzung und Forschungsprogramm des Instituts
B. Forschungsprogramm und Projekte der strafrechtlichen Abteilung
C. Forschungsprogramm und Projekte der kriminologischen Abteilung

\section{AKTUELLE FORSCHUNGSARBEITEN}

A. Überblick

B. Transnationale Kriminalität und territoriales Sicherheitsrecht: Nationale Abschottung im globalen Cyberspace?

C. Terrorismusbekämpfung jenseits der funktionalen Grenzen des Strafrechts: Kontrollverfügungen gegen mutmaßliche Terroristen im Vereinigten Königreich

D. Neue informationstechnische Überwachungsmaßnahmen:

Online-Durchsuchung und Online-Überwachung vor dem Bundesverfassungsgericht

E. Aktuelles Zeitgeschehen:

Steuerermittlungen in Liechtenstein

F. Internationale Kriminalpolitik:

Strafrechtspolitik in China - Auf dem Weg zur Abschaffung der Todesstrafe?

G. Risiko und Risikokontrolle:

Forschungsschwerpunkt Sexualstraftäter

H. Informationsgesellschaft:

Die Überwachung der Telekommunikation

I. Verbrechensfurcht:

Schützt soziale Sicherheit vor Kriminalitätsfurcht?

J. Strafrecht und Kriminologie unter einem Dach:

Grenzen des Rechtsgüterschutzes vor dem Bundesverfassungsgericht

\section{NACHWUCHSFÖRDERUNG}

A. Überblick

B. Ausbildungsbeginn in der International Max Planck Research School for Comparative Criminal Law

C. Vorbereitung der International Max Planck Research School on Retaliation, Mediation and Punishment

D. Beteiligung an der MaxNetAging Research School 
IV. WISSENSCHAFTLICHE ZUSAMMENARBEIT

A. Internationale und nationale Kooperation

B. Ausländische Wissenschaftler am Institut

C. Gutachten

D. Veranstaltungen und Vorträge

E. Lehre

V. ORGA NISATION

A. Forschungsabteilungen

B. Bibliothek

C. Herausgabe von Buchreihen und Zeitschriften

D. Öffentlichkeitsarbeit

124

E. EDV-Dienstleistungen

12)

F. Forschungsförderung

129

G. Fachbeirat und Kuratorium

VI. PERSONALIEN

A. Ehrungen

13:

B. Tätigkeiten

C. Selbstverwaltungsaktivitäten

ANHANG

A. Publikationen

B. Vorträge

C. Lehre

D. Veranstaltungen

E. Doktoranden 



\section{Vorwort}

Der Forschungsbericht des Freiburger MaxPlanck-Instituts für ausländisches und internationales Strafrecht für die Jahre 2006/2007 konzentriert sich auf die neu entwickelten Forschungsaktivitäten. Im Mittelpunkt des neuen Berichts stehen für beide Abteilungen des Instituts das jeweilige Forschungsprogramm, die tabellarische Zusammenfassung der bearbeiteten Projekte sowie ausgewählte Berichte zur Veranschaulichung der Forschungsarbeiten. Ausführlichere Darstellungen zu allen Forschungsprojekten finden sich in zwei separaten Forschungsbroschüren der beiden Abteilungen sowie im WWW. Im Übrigen verweisen wir auf den ausführlichen Überblick zu Geschichte, Organisation, Forschung und sonstigen Aktivitäten im Tätigkeitsbericht 2004/2005.

Im Berichtszeitraum wurden Konzept und Struktur der Forschungsprogramme in den beiden Abteilungen verfeinert. Im Jahr 2007 nahm darüber hinaus die erste International Max Planck Research School for Comparative Criminal Law ihr Ausbildungsprogramm auf; im gleichen Jahr wurde eine zweite Research School on Retaliation, Mediation and Punishment bewilligt, die Anfang 2008 mit der Doktorandenausbildung begann. Die Doktorandenausbildung wurde damit am Institut erstmals in einem strukturierten Rahmen und mit einer übergreifenden Zielsetzung der einzelnen Forschungsarbeiten organisiert, professionalisiert und internationalisiert. Dies wird - auch im Hinblick auf die Gewinnung neuer Nachwuchswissenschaftler - mittel- und langfristig zu einer erheblichen Steigerung der Forschungsleistung führen. Weiter wurden die internationalen Forschungskooperationen besonders im Hinblick auf China, Lateinamerika und ein internationales Netzwerk für eine umfassende internationale Strafrechtsvergleichung ausgebaut. Die Zahl der ausländischen Doktoranden, Institutsmitarbeiter und Gäste nahm weiter zu: Am Institut forschten im Jahr 2007 zusätzlich zu den Doktoranden der Research School insgesamt 242 externe Gastwissenschaftler (2006: 211); die Zahl der Gastwissenschaftler hat sich damit in den letzten vier Jahren mehr als verdoppelt. Die damit verbundenen räumlichen Engpässe wurden dadurch gelöst, dass die Verwaltung in ein neu erworbenes Gebäude neben dem Institut zog und auf diese Weise zusätzlicher Platz für die Doktorandenschulen und Gastwissenschaftler gewonnen wurde. In der strafrechtlichen Abteilung hat darüber hinaus auch die Fertigstellung der noch vor dem Direktorenwechsel begonnenen früheren Projekte gute Fortschritte gemacht, sodass sich die Arbeit im kommenden Berichtszeitraum voll auf das neue Forschungsprogramm konzentrieren kann.

Das Institut erlitt im vergangenen Jahr einen schmerzlichen Verlust: Am 3. September 2007 verstarb der emeritierte Direktor Prof. Dr. Dr. h.c. mult. Günther Kaiser. Günther Kaiser arbeitete bis zu seinem Tod hier im Haus. In seinen letzten Tagen hat er noch einen Beitrag über „Brennpunkte der Wirtschaftskriminologie“ zur Festschrift für seinen Freiburger Kollegen Klaus Tiedemann fertiggestellt. Er war mit den amtierenden und emeritierten Direktoren sowie vielen Mitarbeitern eng verbunden und wegen seiner hohen Fachkompetenz, Bescheidenheit und Liebenswürdigkeit allseits geschätzt. Durch seinen Tod hat das Institut einen großen Verlust erlitten. Wir trauern um ihn und werden unsere Arbeit auf der Grundlage seiner Forschungsergebnisse in seinem Sinne fortführen.

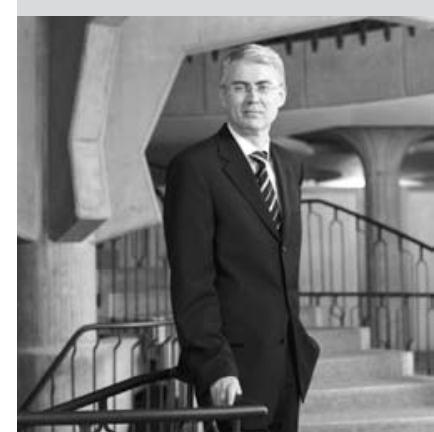

Prof. Dr. Dr. h.c. Ulrich Sieber, Direktor und Leiter der strafrechtlichen Forschungsabteilung am Institut

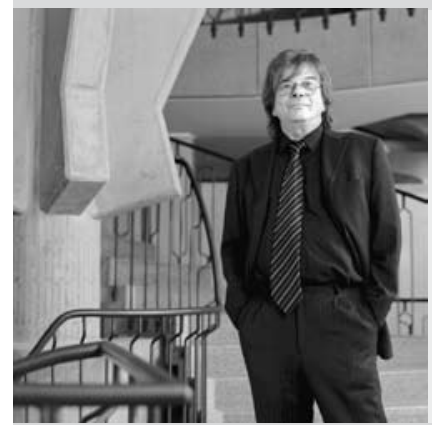

Prof. Dr. Dr. h.c. Hans-Jörg Albrecht, Direktor und Leiter der kriminologischen Forschungsabteilung am Institut

Freiburg, Juni 2008 
In den nachfolgenden Texten werden aus Gründen der Lesefreundlichkeit nicht ständig weibliche und männliche Personenbezeichnungen benutzt. Es sind in allen Fällen Frauen und Männer gleichermaßen gemeint. 


\section{Forschungsprogramm und Forschungsprojekte}




\section{FORSCHUNGSPROGRAMMUND FORSCHUNGSPROJEKTE}

A. Zielsetzung und Forschungsprogramm des Instituts

B. Forschungsprogramm und Projekte der strafrechtlichen Abteilung

C. Forschungsprogramm und Projekte der kriminologischen Abteilung 


\section{A. Zielsetzung und Forschungs- programm des Instituts}

Das Max-Planck-Institut für ausländisches und internationales Strafrecht ist Teil der Max-Planck-Gesellschaft (MPG) und gehört zur Geistes-, Sozial- und Humanwissenschaftlichen Sektion der MPG. Die beiden Direktoren des Instituts sind gleichzeitig wissenschaftliche Mitglieder der Gesellschaft. Die Forschungsausrichtung des Instituts ist grundlagenorientiert und dabei zugleich, als rechts- und sozialwissenschaftliche Forschung, anwendungsbezogen.

Das Institut gliedert sich in die von Prof. Dr. Dr. h.c. Ulrich Sieber geleitete strafrechtliche Abteilung und in die von Prof. Dr. Dr. h.c. Hans-Jörg Albrecht geführte kriminologische Abteilung. Die Fragen des Gesamtinstituts werden von beiden Direktoren gemeinsam entschieden, die sich in der geschäftsführenden Leitung des Instituts alle zwei Jahre abwechseln. Entsprechend den Grundprinzipien der Max-Planck-Gesellschaft bestimmen beide Direktoren ihre Forschungen selbst. Die beiden selbstständigen Forschungsbereiche sind jedoch nicht nur methodisch miteinander verzahnt, sondern auch durch die Wahl ihrer Forschungsgegenstände aufeinander abgestimmt.

In den Forschungsmethoden ergänzen sich die beiden selbstständigen Forschungsabteilungen mit ihren unterschiedlichen Untersuchungsansätzen und theoretischen Perspektiven strafrechtlicher und kriminologischer Forschungen. Der Institutsgründer Prof. Dr. Dr. h.c. mult. Hans-Heinrich Jescheck, der das seit 1947 an der Universität so bezeichnete „Institut für ausländisches und internationales Strafrecht" im Jahr 1966 in die MaxPlanck-Gesellschaft überführt hatte, erläuterte seine Forderung nach „Strafrecht und Kriminologie unter einem Dach" damit, dass Strafrecht ohne Kriminologie „blind“ sei, Kriminologie ohne Strafrecht dagegen ,grenzenlos“.

Im Hinblick auf den Gegenstand der Forschung stehen in beiden Abteilungen seit 2004 im Mittelpunkt der Forschungsprogramme die Herausforderungen für das Strafrecht und die Kriminalpolitik, die sich aus dem gesellschaftlichen Wandel in der „Weltgesellschaft“, der „Informationsgesellschaft" und der „Risikogesellschaft“ ergeben: Die - zunehmend grenzüberschreitend begangene Kriminalität, die Kriminalpolitik und die strafrechtliche Kontrolle verändern sich im Verlauf des
Globalisierungsprozesses. Die Delikte beruhen auf neuen Techniken, Organisationsformen und Risikoszenarien; die neuen Risiken haben eine gesamtgesellschaftliche Bedeutung. Diese Bedeutung entfaltet sich sowohl in empirischer wie in normativer Hinsicht. Das gesamte Spektrum an Veränderungen zeigt sich besonders anschaulich an dem gegenwärtigen globalen Wandel des Strafrechts zu einem präventiven Sicherheitsrecht, das sowohl auf weltweit agierenden Straftätergruppen wie auf weltweit veränderten Wahrnehmungen von Risiken und Kriminalpolitiken jenseits des staatlichen Territoriums beruht. Diese Entwicklung ist deswegen auch nicht mehr allein national zu erklären, sondern nur als Teil eines international zu beobachtenden Prozesses erfassbar.

Das Forschungsprogramm der strafrechtlichen Abteilung zu den Grenzen des Strafrechts beschäftigt sich mit diesen Veränderungen. Vor allem auf der normativen Ebene werden zwei thematische Forschungsschwerpunkte und ein Schwerpunkt zu den einschlägigen Forschungsmethoden gebildet. Der erste Forschungsschwerpunkt zielt auf eine Theorie der internationalen Strafrechtsintegration: Die Herausforderungen, die sich aus dem gesellschaftlichen Wandel der Globalisierung ergeben, führen das klassische nationale Strafrecht an seine territorialen Grenzen. Modelle zur Überwindung dieser territorialen Grenzen des Strafrechts durch ein transnational wirksames Strafrecht sind insbesondere das Europäische Strafrecht und das Völkerstrafrecht. Der zweite Forschungsschwerpunkt der strafrechtlichen Abteilung fokussiert auf eine Theorie zu den funktionalen Grenzen des Strafrechts: Die entsprechenden Herausforderungen für das Strafrecht und die Kriminalpolitik werden besonders an der beschränkten Funktion von Strafrecht deutlich, die mit neuen komplexen Formen der 
Kriminalität, veränderten Risikowahrnehmungen und gewandelten kriminalpolitischen Sicherheitsdiskursen konfrontiert ist (beispielsweise beim Terrorismus, der organisierten Kriminalität, der Wirtschaftskriminalität und der Internetkriminalität). Beide Grenzsituationen hängen in der entstehenden „Weltrisikogesellschaft“ eng zusammen. Um die einschlägigen Fragestellungen methodisch kontrolliert untersuchen und die zukünftigen Lösungen entwickeln zu können, analysiert der dritte Forschungsschwerpunkt der strafrechtlichen Abteilung die für das strafrechtliche Forschungsprogramm zentrale Methodenfrage nach einer Theorie der Strafrechtsvergleichung und einer entsprechenden internationalen Strafrechtsdogmatik.

Im Rahmen der oben beschriebenen grundlegenden gesellschaftlichen Umbrüche und Transformationen der Gegenwart verändern sich nicht nur die Entstehungsbedingungen und Gelegenheitsstrukturen von Kriminalität - und damit die Kriminalität selbst -, sondern auch die gesellschaftlichen Reaktionen, ihre Instrumentarien und Verfahren der Sozialkontrolle und dabei insbesondere die strafrechtliche Sozialkontrolle. Dieser inhaltliche Fokus bestimmt den Zuschnitt und die Ausgestaltung des kriminologischen Forschungsprogramms. Die kriminologische Abteilung untersucht die oben genannten Fragen des sozialen Wandels in fünf Forschungsschwerpunkten: (1) Strafverfahren und Sanktionen im Wandel, (2) Gefährliche Straftäter, (3) Innere Sicherheit, organisierte Kriminalität, Terrorismus - gesellschaftliche Wahrnehmungen und Reaktionen, (4) Kriminalität, soziale Kontexte und sozialer Wandel, (5) Kriminalpolitik und rechtsstaatliche Entwicklung in außereuropäischen und Übergangsgesellschaften. Die Forschung ist methodisch auf die Erfassung von Veränderungen und hiermit auf Längsschnitt- und Wiederholungsuntersuchungen ausgerichtet sowie auf die Entwicklung der Mehrebenenanalyse, mit der die Handlungsebene und soziale Kontexte verknüpft werden. Inhaltlich zielen die Untersuchungen auf die Fortsetzung der am Institut bereits früh begonnenen Implementations- und Evaluations- forschung sowie auf die Fortentwicklung von Theorien der Kriminalität und der strafrechtlichen Sozialkontrolle. Die Projekte zum Strafverfahren greifen dabei insbesondere Fragestellungen neuer und verdeckter Ermittlungsmethoden auf, die - wie die Telekommunikationsüberwachung, die Rasterfahndung, die Überwachung des Wohnraums oder die Verkehrsdatenabfrage - mit neuen Kriminalitätsformen zusammenhängende Risiken und Ermittlungsprobleme beantworten sollen und das sich entwickelnde präventive Sicherheitsrecht repräsentieren. Neue Ermittlungsmethoden sind darüber hinaus auf Fragestellungen der Informationsgesellschaft ausgerichtet, denn sie setzen an Veränderungen der Informationstechnologie an und beziehen sich auf veränderte gesellschaftliche Kommunikationsmuster. Im Rahmen der Untersuchungen zu gefährlichen Straftätern und zur Inneren Sicherheit werden neben der empirischen Erfassung neuer Kriminalitätsphänomene Fragen der Rückfallkriminalität bei Sexualstraftätern, die Prognose sowie die Auswirkungen von Behandlung im Strafvollzug und von Entwicklungen in der Nachentlassungssituation thematisiert. Im Übrigen gilt die Aufmerksamkeit der Wahrnehmung der Sicherheit und den Unsicherheitsgefühlen in der Bevölkerung sowie ihrer Erklärung. Vor allem in Kooperationsprojekten und in Übergangsgesellschaften werden Prozesse der Ausbildung eines rechtsstaatlichen Strafrechts und sein Potential für die (Wieder-)Herstellung sozialer Ordnung ausgeleuchtet.

Dieses Forschungskonzept des Strafrechts und der Kriminologie „unter einem Dach“ zeigt sich nicht nur in den vielfältigen Beziehungen und Ergänzungen zwischen den Forschungsprogrammen beider Abteilungen (vgl. die Projektübersichten in Kapitel I., B.2. und C.2. sowie die ausgewählten Forschungsarbeiten in Kapitel II.). Das Konzept schlägt sich vor allem auch in der Zusammenarbeit beider Abteilungen bei der Ausbildung des wissenschaftlichen Nachwuchses in den am Institut neu gegründeten International Max Planck Research Schools nieder, in denen Doktoranden oft von beiden Direktoren gemeinsam betreut werden (Kapitel III.). 


\section{B. Forschungsprogramm und Projekte der strafrechtlichen Abteilung}

\section{FORSCHUNGSPROGRAMM}

Der Direktorenwechsel am Freiburger Max-Planck-Institut für ausländisches und internationales Strafrecht war in der strafrechtlichen Abteilung Anlass für die Konzeption eines langfristig angelegten Forschungsprogramms, das bereits bei der Feier zum Amtswechsel im Jahr 2004 vorgestellt wurde. Mit den Fortschritten beim Abschluss der noch vor dem Direktorenwechsel begonnenen Projekte konnten sich die Arbeiten der Abteilung im vergangenen Berichtszeitraum zunehmend auf das neue Forschungsprogramm konzentrieren. Dieses zielt auf eine Theoriebildung zu den Zukunftsproblemen des Strafrechts und ist seit 2007 auch für die Ausbildung der Nachwuchswissenschaftler in der International Max Planck Research School for Comparative Criminal Law forschungsleitend. Es soll insbesondere die Forschungserträge der Einzelprojekte zusammenführen und darüber hinausgehende Synergieeffekte und Mehrwerte erzielen, insbesondere bei der Ausbildung des wissenschaftlichen Nachwuchses, bei der gegenseitigen Befruchtung der laufenden Projekte sowie bei der späteren Gesamtbewertung der Einzelergebnisse mit dem Ziel einer umfassenderen Theoriebildung.

\section{Grenzen des Strafrechts}

Gegenstand des Forschungsprogramms sind die Grenzen des Strafrechts. Die aktuelle Verschiebung dieser Grenzen wird an den gesellschaftlichen, wirtschaftlichen und politischen Veränderungen der Welt-, Informations- und Risikogesellschaft deutlich, in der Kriminalität, Kriminalpolitik und Strafrecht einem schnellen Wandel unterliegen. Das Ausmaß der gegenwärtigen Veränderungen im Strafrecht zeigt sich beispielsweise im Europäischen Strafrecht und im Völkerstrafrecht an neuen Formen der internationalen Zusammenarbeit und der Entstehung von Elementen eines echten supranationalen Strafrechts. Ebenso weitreichende Veränderungen manifestieren sich bei der Kontrolle des Terrorismus, die das fundamentale Verhältnis zwischen den Garantien von Sicherheit und Freiheit in Bewegung bringt und klassische politische und rechtliche Kategorien auflöst, wie die Unterscheidung von innerer und äußerer Sicherheit, von Krieg und Verbrechen, von Krieg und Frieden sowie von Strafjustiz, Polizei, Geheimdienst und Militär.

Ziel des Forschungsprogramms ist es, die Grenzen des Strafrechts an den tatsächlichen Veränderungen von Sicherheitsrisiken und Sicherheitsdenken in der sich wandelnden Gesellschaft und den hieraus resultierenden normativen Veränderungen zu untersuchen, um neue Antworten auf die aus die- ser Entwicklung entstehenden kriminalpolitischen Herausforderungen zu entwickeln. Das Programm konzentriert die Forschungen deswegen auf spezifische Forschungsschwerpunkte mit bestimmten Forschungsfragen sowie hierfür besonders ertragversprechenden Forschungsfeldern.

\section{Forschungsschwerpunkte}

Im Mittelpunkt des Forschungsinteresses stehen zwei - miteinander zusammenhängende und für die gegenwärtige Strafrechtsentwicklung fundamentale - Prozesse: (1.) die - mit der Globalisierung - zunehmende Transnationalisierung der Kriminalität sowie (2.) die - mit der Risiko- und Informationsgesellschaft einhergehende - Veränderung der Risiken und der Risikowahrnehmung von komplexen Kriminalitätsformen, insbesondere im Zusammenhang mit Terrorismus, organisierter Kriminalität und Wirtschaftskriminalität. Beide Prozesse führen das klassische Strafrecht sowohl an seine territorialen wie auch an seine funktionalen Grenzen.

Die territorialen Grenzen des Strafrechts und die Möglichkeiten ihrer Überwindung durch ein transnational wirksames Strafrecht bilden den ersten Forschungsschwerpunkt, der auf eine Theorie der internationalen Strafrechtsintegration zielt. Dem liegt die - im Forschungsprogramm näher
Erster Forschungsschwerpunkt: Transnationale Kriminalität, territoriale Grenzen des Strafrechts und internationale Strafrechtsintegration 
Zweiter Forschungsschwerpunkt: Komplexe Kriminalität, funktionale Grenzen des Strafrechts und neue Formen der Sozialkontrolle begründete - Annahme zugrunde, dass die zunehmende transnationale Kriminalität vor allem auf technischen, wirtschaftlichen und politischen Veränderungen der Globalisierung beruht, aus denen sich neue Gelegenheiten zur grenzüberschreitenden Deliktsbegehung, z.B. in internationalen Datennetzen und globalen illegalen Märkten ergeben. Diese neuen Möglichkeiten transnationaler Kriminalität fordern die territorialen Grenzen des nationalstaatlichen Strafrechts heraus, da dieses sich gegen grenzüberschreitende Kriminalität nur schwer durchsetzen lässt, wenn die Geltung seiner Entscheidungen auf anderen Territorien erst langwierige Amts- oder Rechtshilfeverfahren benötigt und die nationalen Strafrechtsordnungen voneinander abweichen. Zur Strafverfolgung der transnationalen Kriminalität ist deswegen nicht nur eine verstärkte Strafrechtsharmonisierung notwendig. Es sind vor allem auch neue Systeme eines transnational wirksamen Strafrechts gefragt, in denen - wie beispielsweise im Europäischen Strafrecht - die klassischen Modelle der zwischenstaatlichen Kooperation und des supranationalen Strafrechts zu hybriden Mischformen und komplexen Mehrebenensystemen der strafrechtlichen Sozialkontrolle weiterentwickelt werden.

Untersuchungen zu diesem ersten Forschungsschwerpunkt betreffen die Wirkungskräfte, Methoden und Modelle der Strafrechtsharmonisierung in dem internationalen Verbundprojekt „Les chemins de l'harmonisation pénale“. Im Europäischen Strafrecht werden in einem größeren Projekt Ziele, Modelle und Systeme von transnational wirksamem Strafrecht mit dem Blick auf die zukünftige Rechtspolitik untersucht; hinzu kommen Untersuchungen zum Prinzip der gegenseitigen Anerkennung von justiziellen Entscheidungen im Europäischen Strafrecht, zur europäischen Strafverteidigung, zu den europäischen Strafprozessordnungen sowie eine systematische Gesamtdarstellung des Europäischen Strafrechts. Auf globaler Ebene werden vor allem das vom Sicherheitsrat der Vereinten Nationen gesetzte und vorgegebene Strafrecht, die Entwicklung von allgemeinen Rechtsgrundsätzen im Völkerstrafrecht und die Kriterien zur Entwicklung völkerstrafrechtlicher Tatbestände analysiert.

Den zweiten Forschungsschwerpunkt bilden die funktionalen Grenzen des Strafrechts und die Möglichkeiten neuer alternativer Maßnahmen der Sozialkontrolle, die in eine Theorie der funktionalen Grenzen des Strafrechts führen. Dem liegt die Annahme zugrunde, dass der technische, wirtschaftliche und politische Wandel der Informations- und der Risikogesellschaft gesteigerte Risiken für die Gesellschaft und eine immer komplexere Kriminalität produziert, die dem Zugriff durch das klassische „Standardprogramm“ des Strafrechts entzogen sind. Dies zeigt sich beispielsweise an dem international arbeitsteiligen Vorgehen weitverzweigter Straftätergruppen, die sich neuer Technologien bedienen, sowie an dem Zerstörungs- und Schadenspotential neuer Formen des Terrorismus, der organisierten Kriminalität, der Wirtschaftskriminalität und der Internetkriminalität. Der damit einhergehende Kontrollverlust des klassischen nationalstaatlichen Strafrechts wird durch neue Netzwerke zwischenstaatlicher Zusammenarbeit, geheime technische Überwachungsmaßnahmen, ein am Präventionsgedanken orientiertes neues "Sicherheitsrecht" unter Einbeziehung polizeirechtlicher, geheimdienstrechtlicher, ausländerrechtlicher und kriegsrechtlicher Maßnahmen (innerhalb und außerhalb des Strafrechts), Mitwirkungspflichten Privater sowie alternative Maßnahmen der Sozialkontrolle (z.B. im Wege der „regulierten Selbstregulierung“ der Wirtschaft) kompensiert und in kriminalpolitischen Sicherheitsdiskursen zur Legitimation von Grenzverschiebungen des Strafrechts benutzt.

Untersuchungen zu diesem zweiten Schwerpunkt erforschen für den Bereich des Terrorismus und der organisierten Kriminalität den präventiven Freiheitsentzug für terroristische „Gefährder“, die einschlägigen neuen Kontrollverfügungen des englischen Strafrechts gegen mutmaßliche Terroristen, das Verbot und die Abgrenzung von Folter, die Terrorismusbekämpfung in China, die internationale strafrechtliche Erfassung von Cyberterrorismus, das neue Sonderstrafrecht gegen organisierte Kriminalität in Frankreich, die Verfolgung von organisierter Kriminalität in Lateinamerika, den Vergleich der Conspiracy (im englischen Strafrecht) mit der Unterstützung von kriminellen Vereinigungen (im deutschen Strafrecht).

Weitere Untersuchungen zur komplexen Kriminalität betreffen im Forschungsfeld der Internetkriminalität die extraterritoriale Strafverfolgung von Cybercrime, Sperrverfügungen im Internet zur nationalen Rechtsdurchsetzung im globalen Cyberspace, die Anonymität im Internet, die strafprozessuale Verfolgung der Datennetzkriminalität, die Online-Durchsuchung, die geheime Lokalisation von Personen, die Verantwortlichkeit für Hyperlinks im Internet sowie eine allgemeine Analyse des Informationsstrafrechts. Hinzu kommen Untersuchungen zum Wirtschaftsstrafrecht über die Inpflichtnahme Privater zum Zwecke der Strafverfolgung und über die kontrollierte Selbstregulierung („Compliance") sowie die Verantwortlichkeit und Sanktionierung von Unternehmen unter besonderer Berücksichtigung von Compliancemaßnahmen. Dies wird im Forschungsfeld Medizinrecht durch eine Untersuchung zur Arzneimittelfälschung und ein Projekt zum Schutz des extrakorporalen Embryos ergänzt. Weitere Arbeiten betreffen übergreifende Fragen wie die Grenzen des Rechtsgüterschutzes oder die Behandlung der massenhaften Kleinkriminalität.

\section{Forschungsmethoden: Insbesondere Strafrechtsvergleichung}

Entsprechend den Zielsetzungen des Forschungsprogramms werden die gesellschaftlichen wie die normativen Bedingungen seines Forschungsgegenstandes untersucht. Demzufolge finden sowohl die empirischen Erhebungsmethoden der 
Sozialwissenschaften als auch die Forschungsmethoden der - insbesondere vergleichenden Strafrechtswissenschaft Anwendung.

Die Analysemethoden der Sozialwissenschaften werden vor allem in der kriminologischen Abteilung des Instituts angewandt. Da die kriminologische Abteilung inhaltlich ähnliche Probleme des sozialen Wandels unter kriminologischen Aspekten untersucht wie die Abteilung Strafrecht unter strafrechtlichen Gesichtspunkten, und da sich die Schwerpunkte beider Abteilungen mit unterschiedlicher Akzentuierung ergänzen, ergeben sich aus den Arbeiten zum Forschungsprogramm der kriminologischen Abteilung Synergieeffekte für das strafrechtliche Programm.

Bei der Lösung der strafrechtlichen Fragen steht die Strafrechtsvergleichung im Vordergrund. Zur Erfassung der gegenwärtigen Rechtsentwicklung ist zunächst eine repräsentative universale Strafrechtsvergleichung erforderlich, da aufgrund der globalen Prozesse in der Kriminal- und Rechtspolitik ein normatives Gesamtbild der aktuellen weltweiten Entwicklungen und ihrer Zusammenhänge notwendig ist, auch um lokale und regionale Entwicklungen zu verstehen. Im Einzelnen ist dafür die Analyse des geltenden Rechts mit einer systematischen und fallbasierten Strafrechtsvergleichung notwendig, die im Wege der wertbasierten Strafrechtsvergleichung auch auf die den Regelungen zugrunde liegenden sozialen und rechtlichen Grundlagen eingeht. Auf dieser Basis sind darüber hinaus allgemeine Rechtsgrundsätze zu entwickeln, die im Wege der wertenden Rechtsvergleichung zur Schließung von Rechtslücken im Europäischen Recht und im Völkerstrafrecht eingesetzt werden können. Unter praktischen Gesichtspunkten unterstützen die so entwickelten Lösungen kriminalpolitischer Probleme eine Politik der „good governance“ und des „benchmarking“ unterschiedlicher kriminalpolitischer Modelle. Unter wissenschaftlichen Gesichtspunkten bietet diese Art der Strafrechtsvergleichung vor allem Lösungen für das grundlegende Wertungsproblem jeder methodisch kontrollierten, d.h. rationalen Kriminalpolitik. Aufgrund dieser Zusammenhänge ist Strafrechtsvergleichung im Forschungsprogramm der strafrechtlichen Abteilung nicht nur eine Forschungsmethode, sondern selbst auch ein zentraler Forschungsgegenstand und Forschungsschwerpunkt.

Um die Voraussetzungen, Methoden und Leistungsfähigkeit der Strafrechtsvergleichung in einen Zusammenhang zu bringen, ist dieser dritte
- methodenorientierte - Forschungsschwerpunkt des Programms auf die Entwicklung einer Theorie der Strafrechtsvergleichung gerichtet. Auf dieser Grundlage soll auch eine universale Strafrechtsdogmatik entwickelt werden. Deren Systembildung, Begriffsbildung und Bestimmung von grundlegenden Werten und Modellen zielen wegen der globalen Herausforderungen und Entwicklungen des Strafrechts auf die Gewinnung von weltweit gültigen Erkenntnissen für international vergleichbare Problemstellungen, die insbesondere in eine „internationale Grammatik des Strafrechts“ und in der Europäischen Union - ein ,gemeineuropäisches Strafrechtssystem" münden.

Diese Methodenfragen der Strafrechtsvergleichung und der Konzeption einer universalen Strafrechtsdogmatik werden im Forschungsprogramm vor allem an einem langfristig angelegten Großprojekt des Instituts entwickelt: dem auch als „virtuelles Institut“ bezeichneten - Max-PlanckInformationssystem für Strafrechtsvergleichung, das auf der Grundlage einer „Max-Planck-Systematik zum Strafrechtsvergleich“ entwickelt wird.

\section{Forschungskonzentration und \\ Forschungskoordination}

Entscheidende Bedeutung für die Umsetzung des Forschungsprogramms haben neben der Bestimmung der Forschungsthemen vor allem auch die Konzentration und die Abstimmung der durchzuführenden Projekte. Die Projekte werden so ausgewählt, dass ihre Erträge in der Addition die Summe der Einzelergebnisse der Untersuchungen übersteigen, um auf diese Weise einen Mehrwert für eine übergreifende Theoriebildung zu den territorialen und funktionalen Grenzen des Strafrechts und zur Strafrechtsvergleichung zu leisten.

Für die Auswahl des Forschungsgegenstandes in einem Projekt ist vor allem der normative Regelungsgegenstand maßgeblich. Die Regelungsgegenstände von Untersuchungen zu den Forschungsschwerpunkten „territoriale“ und „funktionale" Grenzen des Strafrechts werden dabei als „Forschungsfelder“ bezeichnet:

- Für die Theoriebildung zu den territorialen Grenzen des Strafrechts und der Strafrechtsintegration sind Systeme strafrechtlicher Integration notwendig. Diese werden vor allem mit den Forschungsfeldern „Europäisches Strafrecht“ und „Internationales Strafrecht (insbesondere Völkerstrafrecht)“ erfasst. Im Einzelfall einbezogen werden dabei aber auch strafrechtlich relevante Regelungen von speziellen internationalen Organisation, wie z.B. der OECD.
Dritter Forschungsschwerpunkt: Methodenfragen der Strafrechtsvergleichung und intenationale Strafrechtsdogmatik 
n Für die Theoriebildung zu den funktionalen Grenzen des Strafrechts sind Regelungsmaterien von Interesse, deren Komplexität die Tragweite des klassischen Strafrechts übersteigt. Diese werden insbesondere von den Forschungsfeldern „Terrorismus“, „organisierte Kriminalität“, „Wirtschaftskriminalität“, „Computerkriminalität“ und „strafrechtliche Kontrolle der life sciences" erfasst. Die Auswahl des Delinquenzbereichs erfolgt dabei funktional unter dem Aspekt der übergeordneten Forschungsfrage, sodass z.B. bei der organisierten Kriminalität im Hinblick auf entsprechende Fragestellungen auch Völkerstraftaten und Staatskriminalität sowie andere Formen der komplexen Kriminalität einbezogen werden können.

Für die Theoriebildung zur Strafrechtsvergleichung ist demgegenüber alleine die Auswahl des gleichen Regelungsgegenstands in räumlich, zeitlich, politisch bzw. kulturell besonders disparaten oder aber in ähnlichen Rechtsordnungen maßgeblich. Die Projekte betreffen häufig - gewollt - mehrere Forschungsfelder und profitieren dadurch in vielfältiger Weise von den Ergebnissen anderer Arbeiten.

\section{Forschungsprogramm und wissenschaftlicher Nachwuchs}

Neben den Untersuchungen der strafrechtlichen Abteilung ist vor allem auch die Ausbildung des wissenschaftlichen Nachwuchses im Strafrecht in die Forschungsschwerpunkte und Forschungsfelder des Programms eingebettet, insbesondere die Ausbildung in der „International Max Planck Research School for Comparative Criminal Law" (vgl. Kapitel III.B.). Gegenstand der Ausbildung ist hier die Förderung von Promotionen mit strafrechtsvergleichenden oder auf Strafrechtsintegration angelegten Zielsetzungen. Von dieser Einbindung in den Forschungsbetrieb profitieren sowohl die geförderten Nachwuchswissenschaftler wie auch das strafrechtliche Forschungsprogramm, da die Doktoranden mit ihren Untersuchungen einen Beitrag für die Theoriebildung zu den Grenzen des Strafrechts und zur Strafrechtsvergleichung leisten.

\section{Zusammenfassung}

Forschungsgegenstand der strafrechtlichen Abteilung sind die Grenzen des Strafrechts. Diese werden an dem gesellschaftlichen, wirtschaftlichen und politischen Wandel in der Welt-, Informations- und Risikogesellschaft deutlich, die grund- legende Veränderungen der Kriminalität, des Strafrechts und der Kriminalpolitik mit sich bringen.

Forschungsziel der strafrechtlichen Abteilung ist, die Grenzen des Strafrechts an den tatsächlichen Veränderungen von Sicherheitsrisiken und Sicherheitsdenken in einer sich wandelnden Gesellschaft und den entsprechenden normativen Veränderungen zu untersuchen, um neue Antworten auf die aus dieser Entwicklung entstehenden kriminalpolitischen Herausforderungen zu entwickeln. Zur Umsetzung dieses Ziels erfasst das Programm der strafrechtlichen Abteilung die beiden zentralen Herausforderungen der gegenwärtigen Entwicklung für das Strafrecht in zwei Forschungsschwerpunkten und einem Schwerpunkt zur Methodik der Strafrechtswissenschaft:

- Ziel des ersten Forschungsschwerpunkts ist die Theoriebildung zur internationalen Strafrechtsintegration. Zu diesem Zweck werden die in der „Weltgesellschaft“ zunehmende transnationale Kriminalität und ihre Folgen untersucht. Transnationale Kriminalität stellt das klassische nationalstaatliche Strafrecht vor seine territorialen Grenzen und macht die Entwicklung neuer Modelle des transnationalen Strafrechts und der Strafrechtsintegration erforderlich, wie sie gegenwärtig vor allem auf der Ebene der Europäischen Union und der Vereinten Nationen sichtbar sind.

- Ziel des zweiten Forschungsschwerpunkts ist die Theoriebildung zu den funktionalen Grenzen des Strafrechts. Zu diesem Zweck werden die in der „Risiko-“ und „Informationsgesellschaft“ zunehmende komplexe Kriminalität und deren Folgen untersucht. Diese Kriminalität ist vor allem durch neue Risiken und Aufklärungsschwierigkeiten gekennzeichnet, die das klassische liberale Strafrecht an seine funktionalen Grenzen bringt und zu neuen Formen der Sozialkontrolle führt. Damit einher gehen eine gesteigerte Wahrnehmung des Bedrohungspotentials von Kriminalität und kriminalpolitische Sicherheitsdiskurse zur Legitimation von Grenzverschiebungen des Strafrechts.

- Um die zentralen - nur unter einem weltweiten Blickwinkel zu erfassenden - Veränderungen methodisch kontrolliert untersuchen zu können, zielt der dritte Forschungsschwerpunkt auf eine Theoriebildung zur Strafrechtsvergleichung und die Entwicklung einer internationalen Strafrechtsdogmatik.

Zur Umsetzung seiner einzelnen Forschungsziele bündelt das Forschungsprogramm die Un- 
FORSCHUNGSPROGRAMM DER STRAFRECHTLICHEN ABTEILUNG

Auswahl und Konzentration der Forschungsprojekte
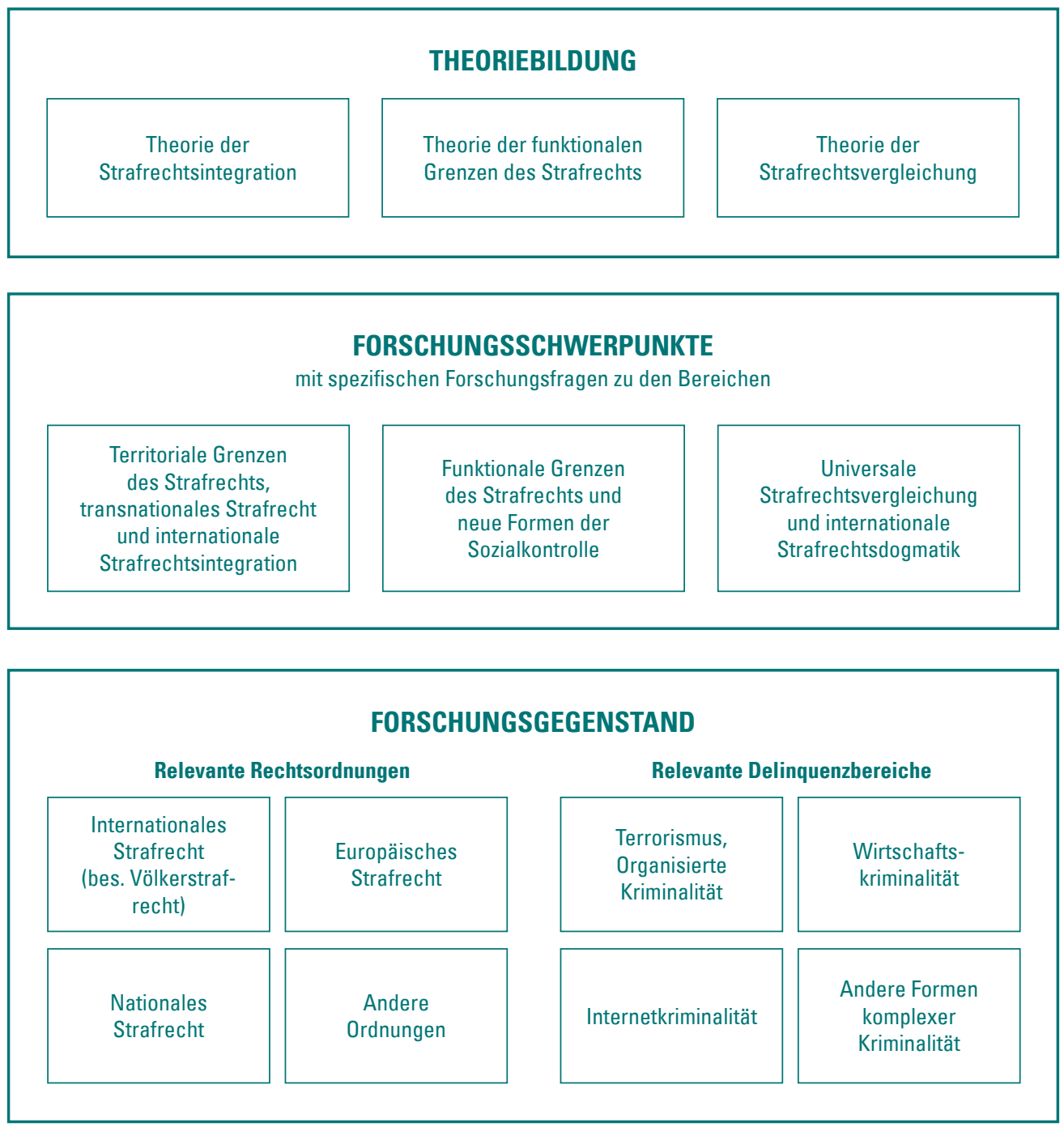

tersuchungen zu den Grenzen des Strafrechts in Forschungsfeldern: Bei der Theoriebildung zur Strafrechtsintegration geht es - neben der allgemeinen Strafrechtsharmonisierung - vor allem um „Europäisches Strafrecht“ und „Völkerstrafrecht“, während die Theoriebildung zu den funktionalen Grenzen des Strafrechts sich auf „Terrorismus“, „organisierte Kriminalität“, „Wirtschaftskriminalität“, und „Internetkriminalität“ konzentriert.
Die obenstehende Skizze verdeutlicht diesen theoriegeleiteten Prozess der Auswahl und Konzentration der Forschungsprojekte, die sowohl durch die zentralen Forschungsfragen als auch durch den für die Analyse relevanten spezifischen Forschungsgegenstand bestimmt werden.

Weiterführende Literaturhinweise: Ulrich Sieber, Grenzen des Strafrechts - Grundlagen und Herausforderungen des strafrechtlichen Forschungsprogramms am Max-Planck-Institut für ausländisches und internationales Strafrecht, ZStW 119 (2007), S. 1-68; ders. Strafrechtsvergleichung im Wandel - Aufgaben, Methoden und Theorieansätze der vergleichenden Strafrechtswissenschaft, in: Sieber/Albrecht (Hrsg.), Strafrecht und Kriminologie unter einem Dach, Berlin 2006, S. 78-130. Siehe dazu auch bereits Ulrich Sieber, Grenzen des Strafrechts, in: Albrecht/Sieber (Hrsg.), Perspektiven der strafrechtlichen Forschung, Amtswechsel am Freiburger MPI 2004, Berlin 2006, S. 35-79, sowie Forschungsbericht 2004/2005, S. $24-37$. 


\section{PROJEKTÜBERSICHT}

Die folgende Aufstellung gibt anhand der Systematik des Forschungsprogramms einen nach den drei Schwerpunkten und ihren Forschungsfeldern geordneten Überblick zu den einzelnen Projekten der strafrechtlichen Abteilung. Soweit - was zur Erreichung von Synergieeffekten angestrebt wird - die Projekte für mehrere Forschungsschwerpunkte und -felder Bedeutung haben, erfolgt die Zuordnung nach dem Hauptziel; weitere Zielsetzungen sind in den Datenfeldern der Projektbeschreibungen angegeben. ${ }^{*}$ Die Aufstellung veranschaulicht dabei mit den entsprechenden Kurzbeschreibungen und Datenfeldern die im Forschungsprogramm erfolgte Konzentration der Untersuchungen durch Angabe der Forschungsschwerpunkte sowie der untersuchten Deliktsbereiche und Rechtsordnungen. Weiter werden der Zeitrahmen der Projekte, ihr Status sowie die Projektkategorie genannt. Die während des Berichtszeitraums bearbeiteten 42 Institutsprojekte verteilen sich - wie im Einzelnen angegeben - auf 20 Promotionsvorhaben, 2 weitere Einzelprojekte von wissenschaftlichen Mitarbeitern (die meist mit externen Partnern in internationalen Projekten kooperieren) sowie 20 Gemeinschaftsprojekte, in denen zwei oder mehr Mitarbeiter zusammenarbeiten. Von diesen Projekten wurden im Berichtszeitraum 13 abgeschlossen.

Erster Forschungsschwerpunkt:

Grenzüberschreitende Kriminalität, territoriale Grenzen des Strafrechts und internationale Strafrechtsintegration

a) Grundlagenfragen

\begin{tabular}{|c|c|c|}
\hline \multicolumn{3}{|c|}{ Grundlagen der Strafrechtsharmonisierung: „Les chemins de l'harmonisation pénale“ } \\
\hline \multicolumn{3}{|c|}{$\begin{array}{l}\text { Prof. Dr. Ulrich Sieber; Prof. Dr. Mireille Delmas-Marty, } \\
\text { Collège de France, Paris; Prof. Dr. Mark Pieth, Universität Basel }\end{array}$} \\
\hline \multicolumn{3}{|c|}{$\begin{array}{l}\text { Die Globalisierung führt zu einer zunehmenden Angleichung der nationalen Strafrechtsordnungen. } \\
\text { Diese Entwicklung verläuft in den verschiedenen Rechtsgebieten jedoch unterschiedlich. Zur Er- } \\
\text { forschung dieser Prozesse untersucht das internationale Gemeinschaftsprojekt mit } 13 \text { Forschern } \\
\text { erstmals Akteure, Wirkungskräfte, Prozesse und Modelle der internationalen Strafrechtsharmoni- } \\
\text { sierung zum Zwecke der Theoriebildung auf der Grundlage von detaillierten Studien zu elf unter- } \\
\text { schiedlichen Rechtsgebieten. }\end{array}$} \\
\hline $\begin{array}{l}\text { Forschungsschwerpunkt(e): } \\
\text { Territoriale Grenzen des Strafrechts; } \\
\text { Strafrechtsvergleichung }\end{array}$ & $\begin{array}{l}\text { Deliktsbereich(e): } \\
\text { Terrorismus; Computerkriminalität; } \\
\text { OK; Wirtschaftskriminalität u.a. }\end{array}$ & $\begin{array}{l}\text { Rechtsordnung(en): } \\
\text { Zahlreiche nationale und interna- } \\
\text { tionale Rechtsordnungen }\end{array}$ \\
\hline $\begin{array}{l}\text { Zeitrahmen: } \\
\text { 2005-2007 }\end{array}$ & $\begin{array}{l}\text { Status: } \\
\text { abgeschlossen }\end{array}$ & $\begin{array}{l}\text { Kategorie: } \\
\text { Institutsprojekt }\end{array}$ \\
\hline
\end{tabular}

* Von den insgesamt 42 aufgelisteten Projekten wurden 7 Projekte noch in der Zeit vor dem Direktorenwechsel im Oktober 2003 begonnen. Diese 7 Projekte wurden so weit wie möglich in das Forschungsprogramm eingegliedert. Sie tragen ebenfalls, teilweise aber nur eingeschränkt, zum neuen Forschungsprogramm bei und sind deswegen in der folgenden Aufstellung bei der Jahresangabe des Projektbeginns mit einem Stern gekennzeichnet. Von diesen 7 Projekten waren zum Ende des Berichtszeitraums 2006/2007 noch 3 Projekte offen, diese sind jedoch weit fortgeschritten und binden im kommenden Berichtszeitraum keine wesentlichen wissenschaftlichen Ressourcen der Abteilung mehr (vgl. demgegenüber Forschungsbericht 2004/2005, S. 20-23). Das noch vor dem Amtswechsel begonnene Projekt „Die Rolle der Ehre im Strafrecht” wurde im Mai 2008 mit der Publikation abgeschlossen. 


\section{b) Europäisches Strafrecht}

\section{Grundlagen, Systeme und Zukunftsperspektiven des Europäischen Strafrechts}

Projektleitung: Prof. Dr. Ulrich Sieber; Dr. Marianne Wade, L.L.B.; Dr. Frank Meyer, LL.M.

Das von der EU geförderte Projekt untersucht Ziele, Systeme und Entwicklungsmöglichkeiten des Europäischen Strafrechts. Seine rechtspolitischen Vorschläge beruhen auf umfassender Grundlagenforschung zum transnational wirksamen Strafrecht: Es analysiert dazu das geltende nationale und supranationale europäische Strafrecht, den europäischen Schutz von Menschenrechten und von institutionellen Garantien sowie die Modelle und Systeme des transnationalen Strafrechts insbes. in Mehrebenensystemen.

Forschungsschwerpunkt(e):

Territoriale Grenzen des Strafrechts

Zeitrahmen:

$2007-2008$
Deliktsbereich(e)

Transnationale Kriminalität; Wirt-

schaftskrim. gegen EU-Interessen
Rechtsordnung(en):

18 europäische Rechtsordnungen,

USA, Nordischer Rat, EU-Recht

Kategorie:

Institutsprojekt

\section{Der Grundsatz der gegenseitigen Anerkennung justizieller Entscheidungen in Strafsachen und seine Umsetzung in Deutschland}

Projektleitung: Thomas Wahl

Der Grundsatz der gegenseitigen Anerkennung strafrechtlicher Entscheidungen ist das Paradebeispiel für die neuen Kooperationsmodelle in der Europäischen Union zur Überwindung der territorialen Grenzen des Strafrechts. Das Projekt erforscht durch eine Analyse der Gesetzgebung und Rechtsprechung die Umsetzungs- und Anwendungsprobleme in Deutschland nach ersten Erfahrungen mit den auf diesem Grundsatz basierenden Mechanismen, vor allem dem Europäischen Haftbefehl.

\begin{tabular}{|c|c|c|}
\hline $\begin{array}{l}\text { Forschungsschwerpunkt(e): } \\
\text { Territoriale Grenzen des Strafrechts }\end{array}$ & $\begin{array}{l}\text { Deliktsbereich(e): } \\
\text { übergreifend }\end{array}$ & $\begin{array}{l}\text { Rechtsordnung(en): } \\
\text { Deutschland; EU-Recht }\end{array}$ \\
\hline $\begin{array}{l}\text { Zeitrahmen: } \\
\text { 2005-2009 }\end{array}$ & $\begin{array}{l}\text { Status: } \\
\text { laufend }\end{array}$ & $\begin{array}{l}\text { Kategorie: } \\
\text { Promotionsprojekt }\end{array}$ \\
\hline
\end{tabular}

\section{Die Implementation des Rahmenbeschlusses zum Europäischen Haftbefehl in den Mitgliedstaaten der Europäischen Union}

Projektleitung: Prof. Dr. Ulrich Sieber; Dr. Peggy Pfützner, LL.M

Das vom T.M.C. Asser Instituut organisierte Projekt analysierte zusammen mit dem Max-PlanckInstitut die Umsetzung des EU-Rahmenbeschlusses über den Europäischen Haftbefehl in allen Mitgliedstaaten der EU. Das Max-Planck-Institut erstellte den deutschen Landesbericht und hatte dadurch frühen Zugriff auf den entsprechenden Gesamtdatenbestand, der später als elektronisches Informationssystem im Internet veröffentlicht wurde. Das Institut war zudem wesentlich an der Abschlusskonferenz des Projekts beteiligt.

\begin{tabular}{|c|c|c|}
\hline $\begin{array}{l}\text { Forschungsschwerpunkt(e): } \\
\text { Territoriale Grenzen des Strafrechts }\end{array}$ & $\begin{array}{l}\text { Deliktsbereich(e): } \\
\text { Organisierte Kriminalität und andere } \\
\text { schwere Kriminalität }\end{array}$ & $\begin{array}{l}\text { Rechtsordnung(en): } \\
25 \text { Mitgliedstaaten der EU und } \\
\text { weitere europ. Staaten; EU-Recht }\end{array}$ \\
\hline $\begin{array}{l}\text { Zeitrahmen: } \\
\text { 2004-2007 }\end{array}$ & $\begin{array}{l}\text { Status: } \\
\text { abgeschlossen }\end{array}$ & $\begin{array}{l}\text { Kategorie: } \\
\text { Institutsprojekt }\end{array}$ \\
\hline
\end{tabular}




\section{Europäische Strafverteidigung}

Projektleitung: Prof. Dr. Jörg Arnold

Bei der Erweiterung der europäischen Strafverfolgung wird das Recht auf Verteidigung nicht gebührend berücksichtigt. Die Untersuchung analysiert deshalb verschiedene Möglichkeiten, die Gesamtbalance im Strafverfahren durch die Schaffung einer „europäischen“ Strafverteidigung zu gewährleisten, und unterbreitet eigene Lösungsvorschläge.

\begin{tabular}{|c|c|c|}
\hline $\begin{array}{l}\text { Forschungsschwerpunkt(e): } \\
\text { Territoriale Grenzen des Strafrechts; } \\
\text { funktionale Grenzen des Strafrechts }\end{array}$ & $\begin{array}{l}\text { Deliktsbereich(e): } \\
\text { übergreifend }\end{array}$ & $\begin{array}{l}\text { Rechtsordnung(en): } \\
\text { Deutschland; EU-Recht }\end{array}$ \\
\hline $\begin{array}{l}\text { Zeitrahmen: } \\
\text { 2007-2009 }\end{array}$ & $\begin{array}{l}\text { Status: } \\
\text { laufend }\end{array}$ & $\begin{array}{l}\text { Kategorie: } \\
\text { Institutsprojekt }\end{array}$ \\
\hline
\end{tabular}

\section{Strafverfahrensrecht in Europa}

Projektleitung: Dr. Barbara Huber; Dr. Richard K. Vogler (University of Sussex, UK)

Die Anwendung von traditionellem Strafverfahrensrecht ist auf das nationale Staatsgebiet beschränkt. Bei der Verfolgung von grenzüberschreitender Kriminalität treten wegen dieser Beschränkung in der Praxis ernsthafte Probleme auf. Die Arbeit stellt der Praxis und der vergleichenden Strafrechtswissenschaft eine aktuelle und übersichtliche Darstellung der Strafprozessrechte repräsentativ ausgewählter Mitgliedsländer der EU in englischer Sprache zur Verfügung.

\begin{tabular}{|c|c|c|}
\hline $\begin{array}{l}\text { Forschungsschwerpunkt(e): } \\
\text { Territoriale Grenzen des Strafrechts }\end{array}$ & $\begin{array}{l}\text { Deliktsbereich(e): } \\
\text { übergreifend }\end{array}$ & $\begin{array}{l}\text { Rechtsordnung(en): } \\
6 \text { europäische Rechtsordnungen }\end{array}$ \\
\hline $\begin{array}{l}\text { Zeitrahmen: } \\
\text { 2004-2007 }\end{array}$ & $\begin{array}{l}\text { Status: } \\
\text { abgeschlossen }\end{array}$ & $\begin{array}{l}\text { Kategorie: } \\
\text { Institutsprojekt }\end{array}$ \\
\hline
\end{tabular}

\section{Systematische Gesamtdarstellung des Europäischen Strafrechts}

Projektleitung: Prof. Dr. Ulrich Sieber; Franz-Hermann Brüner, Europäisches Amt für Betrugsbekämpfung (OLAF); Prof. Dr. Bernd von Heintschel-Heinegg (Bayerisches Oberlandesgericht); Prof. Dr. Helmut Satzger (Universität München)

Die Europäische Union und der Europarat begegnen den neuen Herausforderungen der Globalisierung mit Regelungen, die das nationale Straf- und Strafprozessrecht sowie die Rechtshilfe in Strafsachen stark beeinflussen. Dies hat zu einem verwirrenden Regelwerk geführt, das für Juristen vielfach undurchschaubar geworden ist. Ziel des Projekts ist deswegen eine Systematisierung und Darstellung des europäischen und des europäisierten Strafrechts für weitere wissenschaftliche Arbeiten und für die Praxis.

Forschungsschwerpunkt(e): Territoriale Grenzen des Strafrechts
Deliktsbereich(e):

übergreifend

Strafrecht

Status:

laufend
Kategorie:

Institutsprojekt 


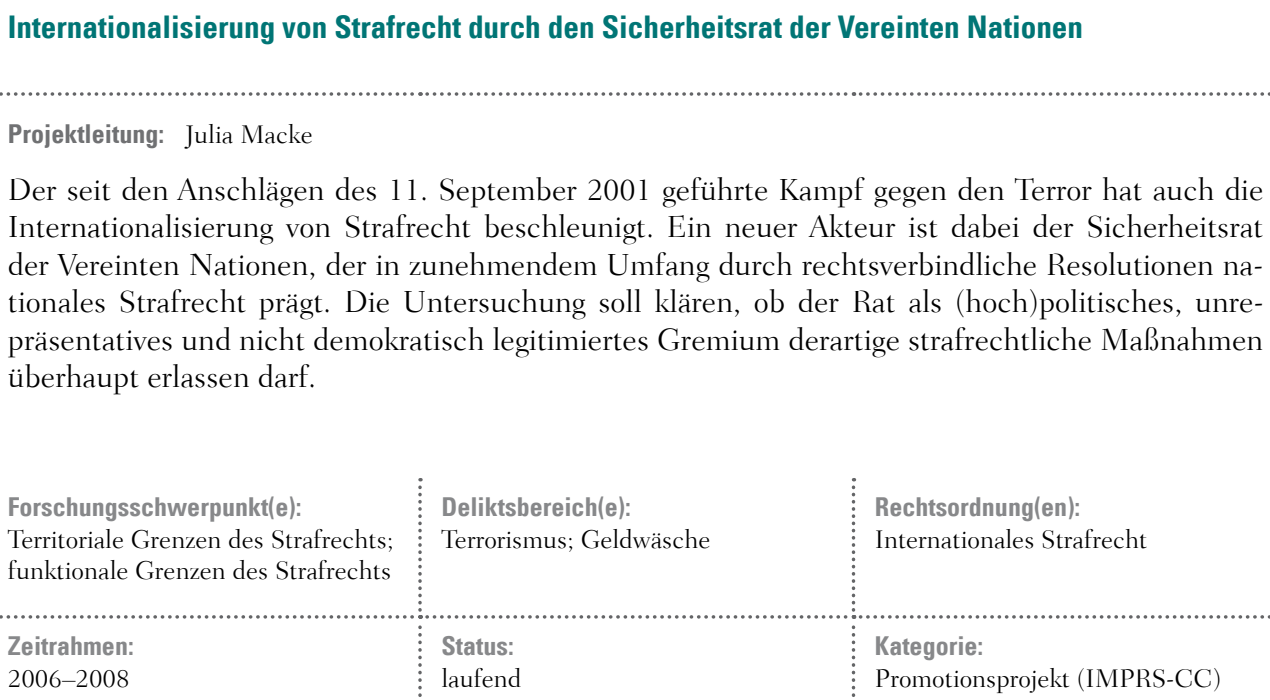

\section{Allgemeine Rechtsgrundsätze des Völkerstrafrechts zur strafbaren Mitwirkung} von Führungspersonen in Straftätergruppen und Netzwerken

Projektleitung: Prof. Dr. Ulrich Sieber; Priv.-Doz. Dr. Hans-Georg Koch; Jan-Michael Simon

Führungspersonen organisierter Straftätergruppen und Netzwerke agieren zumeist im Hintergrund ohne eigenhändige Tatbeteiligung. Das Projekt untersucht, wie 43 Rechtsordnungen mit den dafür typischen Zurechnungs- und Beweisproblemen umgehen. Dabei werden auch neue methodische Wege der Strafrechtsvergleichung erprobt. Ziel ist es, die Entwicklung einschlägiger allgemeiner Rechtsgrundsätze des Völkerstrafrechts zu unterstützen und zur Entwicklung der internationalen Strafrechtsdogmatik beizutragen.

\begin{tabular}{|c|c|c|}
\hline $\begin{array}{l}\text { Forschungsschwerpunkt(e): } \\
\text { Territoriale Grenzen des Strafrechts; } \\
\text { funktionale Grenzen des Strafrechts }\end{array}$ & $\begin{array}{l}\text { Deliktsbereich(e): } \\
\text { Organisierte Kriminalität, insbes. } \\
\text { Völkerstraftaten }\end{array}$ & $\begin{array}{l}\text { Rechtsordnung(en): } \\
43 \text { Rechtsordnungen weltweit; } \\
\text { Völkerstrafrecht }\end{array}$ \\
\hline $\begin{array}{l}\text { Zeitrahmen: } \\
\text { 2005-2008 }\end{array}$ & $\begin{array}{l}\text { Status: } \\
\text { laufend }\end{array}$ & $\begin{array}{l}\text { Kategorie: } \\
\text { Institutsprojekt }\end{array}$ \\
\hline
\end{tabular}

\section{Terrorismus und Völkerstrafrecht}

Projektleitung: Paul Rabbat, LL.M.

Der „neue Terrorismus“, der internationaler und schadensintensiver ist, hat auch im Völkerstrafrecht zur Suche nach der effektivsten Maßnahme geführt. Das Projekt untersucht die Perspektiven für die erfolgreiche Bekämpfung und Bestrafung von Terrorismus durch Völkerstrafrecht. Es analysiert dabei auch die Kriterien für die Schaffung von völkerstrafrechtlichen Tatbeständen.

\begin{tabular}{|c|c|c|}
\hline $\begin{array}{l}\text { Forschungsschwerpunkt(e): } \\
\text { Territoriale Grenzen des Strafrechts; } \\
\text { Funktionale Grenzen des Strafrechts }\end{array}$ & $\begin{array}{l}\text { Deliktsbereich(e): } \\
\text { Terrorismus }\end{array}$ & $\begin{array}{l}\text { Rechtsordnung(en): } \\
\text { Völkerstrafrecht }\end{array}$ \\
\hline $\begin{array}{l}\text { Zeitrahmen: } \\
\text { 2007-2009 }\end{array}$ & $\begin{array}{l}\text { Status: } \\
\text { laufend }\end{array}$ & $\begin{array}{l}\text { Kategorie: } \\
\text { Promotionsprojekt }\end{array}$ \\
\hline
\end{tabular}




\section{Grundlagen und Grenzen völkerrechtlicher Exemtionen}

Projektleitung: Dr. Helmut Kreicker

Das Projekt untersucht, inwieweit völkerrechtliche Immunitäten, Unverletzlichkeitsgarantien und sonstige Exemtionen einer Strafverfolgung durch nationale und internationale Gerichte entgegen stehen. Im Wege einer spezifisch strafrechtlichen Analyse internationaler und nationaler Rechtsprechung, völkerrechtlicher Verträge und staatlicher Gesetzgebung wird das geltende Recht systematisch erfasst und einer kritischen Bewertung unterzogen.

\begin{tabular}{|c|c|c|}
\hline $\begin{array}{l}\text { Forschungsschwerpunkt(e): } \\
\text { Territoriale Grenzen des Strafrechts }\end{array}$ & $\begin{array}{l}\text { Deliktsbereich(e): } \\
\text { Völkerstraftaten/internationale } \\
\text { Verbrechen }\end{array}$ & $\begin{array}{l}\text { Rechtsordnung(en): } \\
\text { Deutschland; Völkerstrafrecht }\end{array}$ \\
\hline $\begin{array}{l}\text { Zeitrahmen: } \\
1999^{*}-2007\end{array}$ & $\begin{array}{l}\text { Status: } \\
\text { abgeschlossen }\end{array}$ & $\begin{array}{l}\text { Kategorie: } \\
\text { Promotionsprojekt }\end{array}$ \\
\hline
\end{tabular}

\section{Nationale Strafverfolgung völkerrechtlicher Verbrechen}

\section{Projektleitung: Prof. Dr. Ulrich Sieber; Prof. Dr. Albin Eser, M.C.J.; Dr. Helmut Kreicker}

Das strafrechtsvergleichende Projekt erforscht, inwieweit die nationalen Strafrechtsordnungen verschiedener Staaten eine Ahndung von Völkerstraftaten durch eigene staatliche Gerichte ermöglichen, welche Defizite gegenüber dem Völkerrecht bestehen und welche Reformen insofern durchgeführt wurden oder geplant sind. Es zeigt, dass die meisten untersuchten Staaten Völkermord und Kriegsverbrechen als solche ahnden können, Verbrechen gegen die Menschlichkeit dagegen nicht.

Forschungsschwerpunkt(e):
Territoriale Grenzen des Strafrechts;
Funktionale Grenzen des Strafrechts

\section{Völkerstrafrecht in Rumänien}

Projektleitung: Dr. Johanna Rinceanu, LL.M

Das Völkerstrafrecht hat sich in den einzelnen Staaten der Welt aufgrund nationaler historischer und politischer Ereignisse unterschiedlich weit entwickelt. Dabei ist ein enger Zusammenhang zwischen diesen Ereignissen sowie dem Wirken von Einzelpersonen in einem bestimmten Land und der dortigen Evolution des Völkerstrafrechts als actio und reactio festzustellen. Die Untersuchung analysiert den Umgang Rumäniens mit dem Völkerstrafrecht in Vergangenheit und Gegenwart.

\begin{tabular}{|c|c|}
\hline $\begin{array}{l}\text { Forschungsschwerpunkt(e): } \\
\text { Territoriale Grenzen des Strafrechts }\end{array}$ & $\begin{array}{l}\text { Deliktsbereich(e): } \\
\text { Völkerstraftaten }\end{array}$ \\
\hline $\begin{array}{l}\text { Zeitrahmen: } \\
2003^{*}-2007\end{array}$ & $\begin{array}{l}\text { Status: } \\
\text { abgeschlossen }\end{array}$ \\
\hline
\end{tabular}

Rechtsordnung(en):
Rumänien; Völkerstrafrecht
Kategorie:
Promotionsprojekt




\section{a) Terrorismus und organisierte Kriminalität}

\begin{tabular}{|c|c|c|}
\hline \multicolumn{3}{|l|}{ Grenzen des Folterverbots } \\
\hline \multicolumn{3}{|c|}{ Projektleitung: Linus Sonderegger, lic. iur. } \\
\hline \multicolumn{3}{|c|}{$\begin{array}{l}\text { Der Kampf gegen den Terror und das Bedürfnis der Gesellschaft nach Sicherheit hat auch vor } \\
\text { dem Tabu des Folterverbots nicht Halt gemacht. Die Untersuchung soll die rechtstatsächlichen } \\
\text { Erscheinungsformen der Folter analysieren und erklären, wo in einer rechtsstaatlichen Demokratie } \\
\text { die Grenzen der Zwangsanwendung zu Verhörzwecken liegen und ob diese Grenzen in Extremsitua- } \\
\text { tionen neu anzupassen sind. }\end{array}$} \\
\hline $\begin{array}{l}\text { Forschungsschwerpunkt(e): } \\
\text { Funktionale Grenzen des Strafrechts }\end{array}$ & $\begin{array}{l}\text { Deliktsbereich(e): } \\
\text { Terrorismus }\end{array}$ & $\begin{array}{l}\text { Rechtsordnung(en): } \\
\text { Deutschland, England und Wales, } \\
\text { USA; Völkerrecht }\end{array}$ \\
\hline $\begin{array}{l}\text { Zeitrahmen: } \\
\text { 2008-2010 }\end{array}$ & $\begin{array}{l}\text { Status: } \\
\text { in Planung }\end{array}$ & $\begin{array}{l}\text { Kategorie: } \\
\text { Promotionsprojekt (IMPRS-CC) }\end{array}$ \\
\hline
\end{tabular}

\section{Präventiver Freiheitsentzug als Instrument der Terrorismusbekämpfung}

Projektleitung: Tim Nikolas Müller, LL.M. Eur., M. Jur.

In einer Vielzahl von Rechtsordnungen sind als Reaktion auf die Terroranschläge der jüngeren Vergangenheit die Möglichkeiten einer präventiven Freiheitsentziehung von Terrorverdächtigen (sog. „Gefährdern“) erweitert oder eingeführt worden. Die Untersuchung analysiert in diesem Zusammenhang die in Deutschland bestehenden Rechtsgrundlagen für eine vorbeugende Freiheitsentziehung und arbeitet die Grenzen heraus, die das Grundgesetz und die Europäische Menschenrechtskonvention einer Entwicklung derartiger Eingriffsgrundlagen auferlegen.

\begin{tabular}{|c|c|c|}
\hline $\begin{array}{l}\text { Forschungsschwerpunkt(e): } \\
\text { Funktionale Grenzen des Strafrechts }\end{array}$ & $\begin{array}{l}\text { Deliktsbereich(e): } \\
\text { Terrorismus }\end{array}$ & $\begin{array}{l}\text { Rechtsordnung(en): } \\
\text { Deutschland }\end{array}$ \\
\hline $\begin{array}{l}\text { Zeitrahmen: } \\
\text { 2007-2009 }\end{array}$ & $\begin{array}{l}\text { Status: } \\
\text { laufend }\end{array}$ & $\begin{array}{l}\text { Kategorie: } \\
\text { Promotionsprojekt (IMPRS-CC) }\end{array}$ \\
\hline
\end{tabular}

\section{Eingriffe in die Bewegungsfreiheit durch die Terrorismusgesetzgebung des Vereinigten Königreichs}

Projektleitung: Susanne Forster, LL.M.

Infolge der Terroranschläge der jüngeren Zeit wird in Deutschland über eine Verschärfung der Anti-Terror-Gesetze diskutiert. Was hierzulande überlegt wird, ist im Vereinigten Königreich zum Teil schon geltendes Recht. Die Untersuchung gibt einen Überblick über die britischen Regelungen und analysiert schwerpunktmäßig die Maßnahmen, mit denen der bei der Terrorismusbekämpfung allgegenwärtige Konflikt zwischen Freiheit und Sicherheit besonders deutlich wird. Siehe dazu II.C.

Forschungsschwerpunkt(e):

Funktionale Grenzen des Strafrechts
Deliktsbereich(e):

Terrorismus
Rechtsordnung(en):

Vereinigtes Königreich

Kategorie:

Promotionsprojekt (IMPRS-CC) 


\section{Terrorismusbekämpfung im Spannungsfeld von Sicherheit und Freiheit} in Deutschland und China

Projektleitung: Zunyou Zhou, LL.M.

Die Anti-Terror-Gesetzgebung hat eine Auseinandersetzung über das Verhältnis von individueller Freiheit und öffentlicher Sicherheit ausgelöst. Die Untersuchung analysiert die jüngsten Entwicklungen in der deutschen und chinesischen Anti-Terror-Gesetzgebung sowie deren praktische Umsetzung. Ziel ist zu klären, inwieweit Deutschland und China ihren Bürgern in diesem Zusammenhang Menschenrechtsschutz gewähren.

\begin{tabular}{|c|c|c|}
\hline $\begin{array}{l}\text { Forschungsschwerpunkt(e): } \\
\text { Territoriale Grenzen des Strafrechts }\end{array}$ & $\begin{array}{l}\text { Deliktsbereich(e): } \\
\text { Terrorismus }\end{array}$ & $\begin{array}{l}\text { Rechtsordnung(en): } \\
\text { Deutschland, China }\end{array}$ \\
\hline $\begin{array}{l}\text { Zeitrahmen: } \\
\text { 2006-2009 }\end{array}$ & $\begin{array}{l}\text { Status: } \\
\text { laufend }\end{array}$ & $\begin{array}{l}\text { Kategorie: } \\
\text { Promotionsprojekt (IMPRS-CC) }\end{array}$ \\
\hline
\end{tabular}

\section{Cyberterrorismus und Harmonisierung der Strafverfolgung}

\section{Projektleitung: Prof. Dr. Ulrich Sieber; Phillip W. Brunst}

Bevorstehende terroristische Angriffe über das Internet wurden häufig mit „Electronic Pearl Harbor“ oder „Digital Waterloo“ betitelt - tatsächliche Angriffe scheinen hingegen bisher weitgehend ausgeblieben zu sein. Das Projekt untersucht im Auftrag des Europarats sowohl die tatsächlichen Chancen und Möglichkeiten, die das Internet Terroristen bietet, als auch die gegenwärtig bestehenden internationalen rechtlichen Instrumente zur Bekämpfung des Cyberterrorismus.

\begin{tabular}{|c|c|c|}
\hline $\begin{array}{l}\text { Forschungsschwerpunkt(e): } \\
\text { Territoriale Grenzen des Strafrechts; } \\
\text { Funktionale Grenzen des Strafrechts }\end{array}$ & $\begin{array}{l}\text { Deliktsbereich(e): } \\
\text { Terrorismus; Computerkriminalität }\end{array}$ & $\begin{array}{l}\text { Rechtsordnung(en): } \\
\text { Europäisches und sonstiges inter- } \\
\text { nationales Strafrecht }\end{array}$ \\
\hline $\begin{array}{l}\text { Zeitrahmen: } \\
\text { 2006-2007 }\end{array}$ & $\begin{array}{l}\text { Status: } \\
\text { abgeschlossen }\end{array}$ & $\begin{array}{l}\text { Kategorie: } \\
\text { Institutsprojekt }\end{array}$ \\
\hline
\end{tabular}

\section{Organisierte Kriminalität im französischen Strafverfahren}

Projektleitung: Dr. Peggy Pfützner, LL.M.

Im Jahr 2004 beschritt der französische Gesetzgeber mit der „Loi Perben II“ einen neuen Weg zur Bekämpfung der organisierten Kriminalität. In einem eigenen Verfahren werden die organisierte Kriminalität erstmals anhand eines Straftatenkatalogs definiert und besondere Strafgerichtszuständigkeiten und Ermittlungsmaßnahmen eingeführt. Das Projekt zeigt insbesondere, wie die Bekämpfung der organisierten Kriminalität zur Einführung eines Verfahrens für allgemeine (mittel)schwere Straftaten führte.

\begin{tabular}{|c|c|c|}
\hline $\begin{array}{l}\text { Forschungsschwerpunkt(e): } \\
\text { Funktionale Grenzen des Strafrechts }\end{array}$ & $\begin{array}{l}\text { Deliktsbereich(e): } \\
\text { Organisierte Kriminalität }\end{array}$ & $\begin{array}{l}\text { Rechtsordnung(en): } \\
\text { Frankreich }\end{array}$ \\
\hline $\begin{array}{l}\text { Zeitrahmen: } \\
\text { 2005-2007 }\end{array}$ & $\begin{array}{l}\text { Status: } \\
\text { abgeschlossen }\end{array}$ & $\begin{array}{l}\text { Kategorie: } \\
\text { Promotionsprojekt }\end{array}$ \\
\hline
\end{tabular}




\section{Mitgliedschaft in einer kriminellen „Vereinigung" und „Conspiracy“} als Modelle zur Strafverfolgung von organisierter Kriminalität

Projektleitung: Almir Maljevič, LL.M.

Verschiedene Erscheinungsformen krimineller Vereinigungen stellen das traditionelle Strafrecht vor große Herausforderungen. Antworten darauf finden sich in den Modellen der "Conspiracy“ und der Teilnahme an einer kriminellen Vereinigung. Das Forschungsvorhaben untersucht, welche Unterschiede und Gemeinsamkeiten diese beiden Modellen aufweisen, wie diese in internationalen Rechtsfiguren kombiniert werden und wie deren Elemente in die Gesetzgebung von Schwellenländern transferiert werden.

\begin{tabular}{|c|c|c|}
\hline $\begin{array}{l}\text { Forschungsschwerpunkt(e): } \\
\text { Funktionale Grenzen des Strafrechts }\end{array}$ & $\begin{array}{l}\text { Deliktsbereich(e): } \\
\text { Organisierte Kriminalität; } \\
\text { Terrorismus }\end{array}$ & $\begin{array}{l}\text { Rechtsordnung(en): } \\
5 \text { europäische Rechtsordnungen; } \\
\text { Europäisches und UN-Strafrecht }\end{array}$ \\
\hline $\begin{array}{l}\text { Zeitrahmen: } \\
\text { 2006-2008 }\end{array}$ & $\begin{array}{l}\text { Status: } \\
\text { laufend }\end{array}$ & $\begin{array}{l}\text { Kategorie: } \\
\text { Promotionsprojekt (IMPRS-CC) }\end{array}$ \\
\hline
\end{tabular}

\section{Strafrecht und Gacaca: Die Aufarbeitung des ruandischen Völkermords mit einem pluralistischen Rechtsmodell}

\section{Projektleitung: Nandor Knust}

Auf systematische Massengewalt wird mit unterschiedlichen rechtsförmigen Verfahren reagiert, seien sie staatlicher, nichtstaatlicher oder gemischt staatlich-nichtstaatlicher Natur. Die Forschungsarbeit untersucht daher die Verfahren des UN-Ruanda Strafgerichtshofs, der ruandischen Strafgerichtsbarkeiten und der neo-traditionellen Gacaca Gerichtsbarkeiten im Umgang mit der ruandischen Massengewalt und klärt die Frage nach einem pluralistischen Ansatz zur Verfolgung von Völkerstraftaten.

\begin{tabular}{|c|c|c|}
\hline $\begin{array}{l}\text { Forschungsschwerpunkt(e): } \\
\text { Funktionale Grenzen des Strafrechts }\end{array}$ & $\begin{array}{l}\text { Deliktsbereich(e): } \\
\text { Organisierte Kriminalität, insbes. } \\
\text { Völkerstraftaten }\end{array}$ & $\begin{array}{l}\text { Rechtsordnung(en): } \\
\text { Völkerstrafrecht, Nationales } \\
\text { Strafecht, Stammesrecht }\end{array}$ \\
\hline $\begin{array}{l}\text { Zeitrahmen: } \\
\text { 2005-2009 }\end{array}$ & $\begin{array}{l}\text { Status: } \\
\text { laufend }\end{array}$ & $\begin{array}{l}\text { Kategorie: } \\
\text { Promotionsprojekt (IMPRS-CC) }\end{array}$ \\
\hline
\end{tabular}

\section{b) Cybercrime}

\section{Extraterritoriale Strafverfolgung von Cybercrime}

\section{Projektleitung: Prof. Dr. Ulrich Sieber}

Cybercrime ist ein Prototyp der grenzüberschreitenden Kriminalität. Die Reaktionen auf diese Delikte in weltweiten Datennetzen zeigen deswegen besonders deutlich die Versuche der Nationalstaaten, die nationalen Grenzen durch eine extraterritoriale Ausdehnung des Strafrechts zu überwinden. Das Projekt macht die Grenzen eines solchen Ansatzes deutlich: Er ist nur in Einzelbereichen und Einzelfällen erfolgreich, führt jedoch zu keinem allgemeinen Lösungsmodell für die Entwicklung eines transnational wirksamen Strafrechts.

Forschungsschwerpunkt(e):

Territoriale Grenzen des Strafrechts;

funktionale Grenzen des Strafrechts
Deliktsbereich(e)

Computerkriminalität
Rechtsordnung(en):

Zahlreiche Rechtsordnungen weltweit

Status:

abgeschlossen

Institutsprojekt 


\section{Nationale Abschottung durch Sperrverfügungen gegen illegale Inhalte im Internet}

Projektleitung: Prof. Dr. Ulrich Sieber; Malaika Nolde, LL.M.

Die Nationalstaaten gehen gegen illegale Inhalte im Internet nicht nur durch eine exterritoriale Ausdehnung ihres Strafrechts vor, sondern auch mit dem Versuch, das Netz gegen illegale Inhalte im Ausland abzuschotten. Das Projekt zeigt, dass derartige „Sperrverfügungen“ gegen Accessprovider auf dem eigenen Hoheitsgebiet einen Zugriff der Bürger auf illegale Inhalte im Internet nur in begrenztem Maße und nur mit intensiven Freiheitseingriffen (insb. in das Fernmeldegeheimnis) verhindern können. Das Projekt ist in Kapitel II.D. näher dargestellt.

\begin{tabular}{|c|c|c|}
\hline $\begin{array}{l}\text { Forschungsschwerpunkt(e): } \\
\text { Territoriale Grenzen des Strafrechts; } \\
\text { funktionale Grenzen des Strafrechts }\end{array}$ & $\begin{array}{l}\text { Deliktsbereich(e): } \\
\text { Computerkriminalität }\end{array}$ & $\begin{array}{l}\text { Rechtsordnung(en): } \\
\text { Deutschland }\end{array}$ \\
\hline $\begin{array}{l}\text { Zeitrahmen: } \\
\text { 2006-2008 }\end{array}$ & $\begin{array}{l}\text { Status: } \\
\text { laufend }\end{array}$ & $\begin{array}{l}\text { Kategorie: } \\
\text { Institutsprojekt }\end{array}$ \\
\hline
\end{tabular}

\section{Herausforderungen und Grenzen der Strafverfolgung von Internetkriminalität}

Projektleitung: Jan Spoenle

Das rasante Wachstum der Internetkriminalität wirft die Frage nach der Reaktion der Strafverfolgungsbehörden auf. Die Untersuchung lokalisiert die rechtlichen und rechtstatsächlichen Probleme der Strafverfolgungspraxis. Durch die Analyse von Ermittlungshindernissen sollen Lösungen für eine effektivere Bekämpfung dieser Kriminalität erarbeitet und mit den spezifischen Gefährdungen zentraler Freiheitsrechte der Informations- und Kommunikationsgesellschaft abgewogen werden.

\begin{tabular}{|c|c|c|}
\hline $\begin{array}{l}\text { Forschungsschwerpunkt(e): } \\
\text { Territoriale Grenzen des Strafrechts; } \\
\text { funktionale Grenzen des Strafrechts }\end{array}$ & $\begin{array}{l}\text { Deliktsbereich(e): } \\
\text { Computerkriminalität; Organisierte } \\
\text { Kriminalität }\end{array}$ & $\begin{array}{l}\text { Rechtsordnung(en): } \\
\text { Deutschland }\end{array}$ \\
\hline $\begin{array}{l}\text { Zeitrahmen: } \\
\text { 2007-2009 }\end{array}$ & $\begin{array}{l}\text { Status: } \\
\text { laufend }\end{array}$ & $\begin{array}{l}\text { Kategorie: } \\
\text { Promotionsprojekt }\end{array}$ \\
\hline
\end{tabular}

\section{Reichweite und Grenzen der Anonymität im Internet}

Projektleitung: Phillip W. Brunst

Das Projekt untersucht die rechtlichen und tatsächlichen Rahmenbedingungen für eine anonyme Kommunikation im Internet, ihre Durchsetzbarkeit sowie Optionen der Re-Identifikation. Es betrifft damit gleichermaßen die Forschungsfragen des Instituts nach den funktionalen und den territorialen Grenzen des Strafrechts. Das Projekt leistet damit einen Beitrag zu der datenschutzrechtlichen Diskussion um das „Recht auf Anonymität“.

\begin{tabular}{|c|c|c|}
\hline $\begin{array}{l}\text { Forschungsschwerpunkt(e): } \\
\text { Territoriale Grenzen des Strafrechts; } \\
\text { funktionale Grenzen des Strafrechts }\end{array}$ & $\begin{array}{l}\text { Deliktsbereich(e): } \\
\text { Computerkriminalität }\end{array}$ & $\begin{array}{l}\text { Rechtsordnung(en): } \\
\text { Deutschland; europäisches Recht }\end{array}$ \\
\hline $\begin{array}{l}\text { Zeitrahmen: } \\
\text { 2003-2008 }\end{array}$ & $\begin{array}{l}\text { Status: } \\
\text { laufend }\end{array}$ & $\begin{array}{l}\text { Kategorie: } \\
\text { Promotionsprojekt }\end{array}$ \\
\hline
\end{tabular}




\section{Erstellung von Bewegungsprofilen zur Strafverfolgung}

Projektleitung: René M. Kieselmann

Strafverfolgungsbehörden nutzen diverse Überwachungsmöglichkeiten, um Personen zu orten und Bewegungsbilder zu erstellen. Der „gläserne Bürger“ wird durch Digitalisierung, Datenspeicherung und Datenverknüpfung zur Realität. Das Projekt analysiert Technik und rechtstatsächliche Befunde. Es werden strafprozessuale Regelungsmöglichkeiten dargestellt, welche die rasante technische Entwicklung erfassen und dabei rechtsstaatliche und verfassungsrechtliche Grundlagen berücksichtigen.

\begin{tabular}{|c|c|c|}
\hline $\begin{array}{l}\text { Forschungsschwerpunkt(e): } \\
\text { Funktionale Grenzen des Strafrechts }\end{array}$ & $\begin{array}{l}\text { Deliktsbereich(e): } \\
\text { übergreifend }\end{array}$ & $\begin{array}{l}\text { Rechtsordnung(en): } \\
\text { Deutschland }\end{array}$ \\
\hline $\begin{array}{l}\text { Zeitrahmen: } \\
\text { 2005-2008 }\end{array}$ & $\begin{array}{l}\text { Status: } \\
\text { laufend }\end{array}$ & $\begin{array}{l}\text { Kategorie: } \\
\text { Promotionsprojekt }\end{array}$ \\
\hline
\end{tabular}

\section{Systematische Analyse der Deliktsformen und Risiken der Computerkriminalität}

Projektleitung: Prof. Dr. Ulrich Sieber; Phillip W. Brunst; Nadine Gröseling

Aufgrund der Transnationalität von Angriffen über Computer und Datennetze sind die Sicherheit der Informationstechnik sowie die Prävention und Verfolgung von Computer- und Datennetzkriminalität nicht nur für den Einzelnen, sondern auch für die internationale Gemeinschaft von großer Bedeutung. Den neuen Herausforderungen können sich Normgeber und -anwender jedoch nur erfolgreich stellen, wenn sie die Bedrohungslage und deren Risiken kennen. Hierzu erfolgt eine explorative Studie.

\begin{tabular}{|c|c|c|}
\hline $\begin{array}{l}\text { Forschungsschwerpunkt(e): } \\
\text { Territoriale Grenzen des Strafrechts; } \\
\text { funktionale Grenzen des Strafrechts }\end{array}$ & $\begin{array}{l}\text { Deliktsbereich(e): } \\
\text { Computerkriminalität }\end{array}$ & $\begin{array}{l}\text { Rechtsordnung(en): } \\
\text { keine (empirische Studie) }\end{array}$ \\
\hline $\begin{array}{l}\text { Zeitrahmen: } \\
\text { 2006-2009 }\end{array}$ & $\begin{array}{l}\text { Status: } \\
\text { laufend }\end{array}$ & $\begin{array}{l}\text { Kategorie: } \\
\text { Institutsprojekt }\end{array}$ \\
\hline
\end{tabular}

\section{Strafrechtliche Verantwortlichkeit für Hyperlinks im Internet}

Projektleitung: Frank Michael Höfinger

Im Worldwide Web kann ein Informationsanbieter Verknüpfungen (Hyperlinks) zu jeder anderen Information weltweit herstellen, auch zu strafbaren Inhalten. Das Projekt untersucht die dadurch aufgeworfenen Fragen aus dem allgemeinen und dem besonderen Teil des Strafrechts, und dabei vor allem, wann die Verlinkung strafbarer Inhalte straflos bleibt. Hiervon sind auch die territorialen und die funktionalen Grenzen des Strafrechts im Hinblick auf die Meinungsfreiheit betroffen.

\begin{tabular}{|c|c|c|}
\hline $\begin{array}{l}\text { Forschungsschwerpunkt(e): } \\
\text { Territoriale Grenzen des Strafrechts; } \\
\text { funktionale Grenzen des Strafrechts }\end{array}$ & $\begin{array}{l}\text { Deliktsbereich(e): } \\
\text { Computerkriminalität }\end{array}$ & $\begin{array}{l}\text { Rechtsordnung(en): } \\
\text { Deutschland }\end{array}$ \\
\hline $\begin{array}{l}\text { Zeitrahmen: } \\
\text { 2005-2008 }\end{array}$ & $\begin{array}{l}\text { Status: } \\
\text { laufend }\end{array}$ & $\begin{array}{l}\text { Kategorie: } \\
\text { Promotionsprojekt }\end{array}$ \\
\hline
\end{tabular}




\section{Betrug bei Online-Auktionen}

Projektleitung: Andreas Dingler

Der Wandel von herkömmlichen Auktionen zu Online-Auktionen zeigt prototypisch die Veränderungen von klassischen Deliktsformen unter den Bedingungen der globalen Vernetzung. Das Dissertationsprojekt analysiert die neu entstandene Kriminalität und die - insb. in der Anonymität des Internet liegenden - Gründe von Risikoveränderungen und Risikosteigerungen. Die Arbeit macht weiter deutlich, dass die neuen Deliktsformen im Wesentlichen vom klassischen Betrugsstrafrecht erfasst werden.

\begin{tabular}{|c|c|c|}
\hline $\begin{array}{l}\text { Forschungsschwerpunkt(e): } \\
\text { Funktionale Grenzen des Strafrechts }\end{array}$ & $\begin{array}{l}\text { Deliktsbereich(e): } \\
\text { Computerkriminalität }\end{array}$ & $\begin{array}{l}\text { Rechtsordnung(en): } \\
\text { Deutschland }\end{array}$ \\
\hline $\begin{array}{l}\text { Zeitrahmen: } \\
\text { 2003-2007 }\end{array}$ & $\begin{array}{l}\text { Status: } \\
\text { abgeschlossen }\end{array}$ & $\begin{array}{l}\text { Kategorie: } \\
\text { Promotionsprojekt }\end{array}$ \\
\hline
\end{tabular}

\section{Reform des Computerstrafrechts in Deutschland}

Projektleitung: Nadine Gröseling; Frank Michael Höfinger

Der strafrechtliche Schutz von Computerdaten und Computersystemen wurde im Jahr 2007 durch das 41. StrÄndG umfassend umgestaltet. Das Projekt untersucht die dogmatischen und praktischen Implikationen der Reform. Es zeigt auf, wo die erklärten Ziele des Änderungsgesetzgebers nicht erreicht wurden, neue Formen komplexer Computerkriminalität sachgerecht zu erfassen und die einschlägigen Vorgaben des Europarats und der EU umzusetzen.

\begin{tabular}{|c|c|c|}
\hline $\begin{array}{l}\text { Forschungsschwerpunkt(e): } \\
\text { Funktionale Grenzen des Strafrechts }\end{array}$ & $\begin{array}{l}\text { Deliktsbereich(e): } \\
\text { Computerkriminalität }\end{array}$ & $\begin{array}{l}\text { Rechtsordnung(en): } \\
\text { Deutschland; Europäisches Recht }\end{array}$ \\
\hline $\begin{array}{l}\text { Zeitrahmen: } \\
\text { 2007-2008 }\end{array}$ & $\begin{array}{l}\text { Status: } \\
\text { laufend }\end{array}$ & $\begin{array}{l}\text { Kategorie: } \\
\text { Institutsprojekt }\end{array}$ \\
\hline
\end{tabular}

\section{c) Wirtschaftskriminalität}

\section{Inpflichtnahme der Privatwirtschaft zur Strafverfolgungsvorsorge}

\section{Projektleitung: Malaika Nolde, LL.M.}

Die zunehmende Ausdehnung des Strafverfahrens weit in das Vorfeld des Anfangsverdachts führt den Staat an die Grenzen eigener Ressourcen. Als Konsequenz wird die traditionelle Bewertung von Repression als genuine Staatsaufgabe in Frage gestellt. Seit Beginn der 90er Jahre findet ein „Outsourcing“ der Strafverfolgungsvorsorge durch die Inpflichtnahme der Privatwirtschaft statt. Das Projekt überprüft diese Entwicklung unter Einbeziehung staatstheoretischer und individualrechtlicher Aspekte.

\begin{tabular}{|c|c|c|}
\hline $\begin{array}{l}\text { Forschungsschwerpunkt(e): } \\
\text { Funktionale Grenzen des Strafrechts }\end{array}$ & $\begin{array}{l}\text { Deliktsbereich(e): } \\
\text { Computerkriminalität; Wirtschafts- } \\
\text { kriminalität }\end{array}$ & $\begin{array}{l}\text { Rechtsordnung(en): } \\
\text { Deutschland }\end{array}$ \\
\hline $\begin{array}{l}\text { Zeitrahmen: } \\
\text { 2005-2008 }\end{array}$ & $\begin{array}{l}\text { Status: } \\
\text { laufend }\end{array}$ & $\begin{array}{l}\text { Kategorie: } \\
\text { Promotionsprojekt }\end{array}$ \\
\hline
\end{tabular}




\section{Verantwortlichkeit und Sanktionierung von Unternehmen unter besonderer Berücksichtigung von Compliance-Maßnahmen}

Projektleitung: Marc Engelhart

Fälle wie der Bestechungsskandal bei Siemens werfen die Frage nach einer Sanktionierung von Unternehmen und der Notwendigkeit von unternehmensinternen Maßnahmen zur Vermeidung von Kriminalität auf. Die Untersuchung analysiert das deutsche und das US-amerikanische Unternehmenssanktionsrecht unter besonderer Berücksichtigung von neuen Formen der „regulierten Selbstregulierung" (insbesondere sogenannte Compliance-Maßnahmen). Dabei werden Ansatzpunkte für eine Reform des deutschen Unternehmenssanktionsrechts entwickelt.

\begin{tabular}{|c|c|c|}
\hline $\begin{array}{l}\text { Forschungsschwerpunkt(e): } \\
\text { Funktionale Grenzen des Strafrechts }\end{array}$ & $\begin{array}{l}\text { Deliktsbereich(e): } \\
\text { Wirtschaftskriminalität }\end{array}$ & $\begin{array}{l}\text { Rechtsordnung(en): } \\
\text { Deutschland, USA }\end{array}$ \\
\hline $\begin{array}{l}\text { Zeitrahmen: } \\
\text { 2005-2009 }\end{array}$ & $\begin{array}{l}\text { Status: } \\
\text { laufend }\end{array}$ & $\begin{array}{l}\text { Kategorie: } \\
\text { Promotionsprojekt (IMPRS-CC) }\end{array}$ \\
\hline
\end{tabular}

d) Grundfragen der Life Sciences und der Medizin

Möglichkeiten und Grenzen des Strafrechts gegen Arzneimittelfälschungen

Projektleitung: Priv.-Doz. Dr. Hans-Georg Koch

Gefälschte Arzneimittel bedrohen Leben und Gesundheit ihrer Anwender. Sie sind damit weit mehr als nur Produktpiraterie gegenüber Originalherstellern. Das rechtsvergleichende Projekt erforscht, mit welchen Mitteln in unterschiedlicher Weise betroffene Länder den Arzneimittelsektor vor gefälschten Produkten schützen und wie sie einschlägige Straftaten der zunehmend international agierenden Täter bekämpfen.

\begin{tabular}{|c|c|c|}
\hline $\begin{array}{l}\text { Forschungsschwerpunkt(e): } \\
\text { Territoriale Grenzen des Strafrechts; } \\
\text { funktionale Grenzen des Strafrechts }\end{array}$ & $\begin{array}{l}\text { Deliktsbereich(e): } \\
\text { Medizin- und Biostraftaten; } \\
\text { Wirtschaftskriminalität }\end{array}$ & $\begin{array}{l}\text { Rechtsordnung(en): } \\
12 \text { Rechtsordnungen weltweit }\end{array}$ \\
\hline $\begin{array}{l}\text { Zeitrahmen: } \\
\text { 2006-2008 }\end{array}$ & $\begin{array}{l}\text { Status: } \\
\text { laufend }\end{array}$ & $\begin{array}{l}\text { Kategorie: } \\
\text { Institutsprojekt }\end{array}$ \\
\hline
\end{tabular}

\section{Der Status des extrakorporalen Embryos}

Projektleitung: Prof. Dr. Albin Eser, M.C.J.; Priv.-Doz. Dr. Hans-Georg Koch

Das interdisziplinäre Verbundprojekt untersucht rechtsvergleichend, welches Maß an (straf-)rechtlichem Schutz Rechtsordnungen mit unterschiedlichem soziokulturellem Hintergrund den In-vitroEmbryonen gewähren bzw. welche (kontrollierten?) Freiräume sie der fortpflanzungsmedizinischen Praxis und der embryobasierten Forschung einräumen. Es werden Möglichkeiten und Grenzen internationaler Konsensfindung aufgezeigt und Ansätze zum legislativen Umgang mit verbleibenden Divergenzen entwickelt.

\begin{tabular}{|c|c|c|}
\hline $\begin{array}{l}\text { Forschungsschwerpunkt(e): } \\
\text { Territoriale Grenzen des Strafrechts; } \\
\text { funktionale Grenzen des Strafrechts }\end{array}$ & $\begin{array}{l}\text { Deliktsbereich(e): } \\
\text { Medizin- und Biostraftaten }\end{array}$ & $\begin{array}{l}\text { Rechtsordnung(en): } \\
\text { Zahlreiche Rechtsordnungen } \\
\text { weltweit }\end{array}$ \\
\hline $\begin{array}{l}\text { Zeitrahmen: } \\
2002^{*}-2007\end{array}$ & $\begin{array}{l}\text { Status: } \\
\text { abgeschlossen }\end{array}$ & $\begin{array}{l}\text { Kategorie: } \\
\text { Institutsprojekt }\end{array}$ \\
\hline
\end{tabular}


e) Übergreifende Fragen

Retaliation-Mediation-Punishment (Vergeltung-Mediation-Bestrafung)

Projektleitung: Jan-Michael Simon; Dr. Pablo Galain Palermo

Das Projekt untersucht die Grenzen des Strafrechts hinsichtlich seiner identitätsstiftenden Funktion und Steuerungsfähigkeit von Gesellschaft. Feststellungen zu dieser Funktion von Strafe sind bereits bei E. Durkheim zu finden. Speziell für das staatliche Strafrecht fehlt es aber bislang an Untersuchungen. Vor allem auch angesichts einer zusammenrückenden Welt, in der unterschiedliche Kulturen in einem staatlichen Raum zusammentreffen, soll diese Forschungslücke geschlossen werden.

Forschunerpunkt(e): Funktionale Grenzen des Strafrechts

Deliktsbereich(e):

Organisierte Kriminalität; Terrorismus; gewalttätige Sozialkonflikte

Zeitrahmen:

2006-2009
Rechtsordnung(en):

10 lateinamerikanische

Rechtsordnungen

Kategorie:

Institutsprojekt

\section{Strafrecht in Reaktion auf Systemunrecht}

Projektleitung: Prof. Dr. Albin Eser; Prof. Dr. Ulrich Sieber; Prof. Dr. Jörg Arnold (Projektkoordinator)

Das Projekt erforscht Funktion und Grenzen des Strafrechts bei der Ablösung vordemokratischer politischer Systeme und der Verarbeitung von Systemunrecht des alten Sytems in Transitions- bzw. Transformationsgesellschaften. Im Ergebnis lässt sich kein „Königsweg“ des strafrechtlichen Umgangs mit der Vergangenheit nach politischen Systemwechseln erkennen. Der einzelne Weg des Transitionsstrafrechts hängt von der länderspezifischen Vergangenheitspolitik in ihrem konkreten historischen Kontext ab.

\begin{tabular}{|c|c|c|}
\hline $\begin{array}{l}\text { Forschungsschwerpunkt(e): } \\
\text { Funktionale Grenzen des Strafrechts }\end{array}$ & $\begin{array}{l}\text { Deliktsbereich(e): } \\
\text { Schwere Menschenrechtsverlet- } \\
\text { zungen (Staatskriminalität) }\end{array}$ & $\begin{array}{l}\text { Rechtsordnung(en): } \\
23 \text { Länder weltweit; } \\
\text { Völkerstrafrecht }\end{array}$ \\
\hline $\begin{array}{l}\text { Zeitrahmen: } \\
1996^{*}-2008\end{array}$ & $\begin{array}{l}\text { Status: } \\
\text { laufend }\end{array}$ & $\begin{array}{l}\text { Kategorie: } \\
\text { Institutsprojekt }\end{array}$ \\
\hline
\end{tabular}

\section{Grenzen des Rechtsgüterschutzes}

\section{Projektleitung: Prof. Dr. Ulrich Sieber; Dr. Konstanze Jarvers}

Anlässlich eines Gutachtens für das Bundesverfassungsgericht untersucht die strafrechtliche Abteilung die strafrechtlichen Aspekte einvernehmlicher sexueller Handlungen zwischen erwachsenen Verwandten. Der rechtsvergleichende Teil analysiert die einschlägigen Grenzen des Rechtsgüterschutzes in den Rechtsordnungen von 22 Ländern, die teils in unterschiedlicher Ausgestaltung eine Inzeststrafbarkeit vorsehen, teils aber auch ohne eine solche auskommen. Das gemeinsam mit der kriminologischen Abteilung durchgeführte Gesamtprojekt wird unter Kapitel II.J. näher beschrieben.

\begin{tabular}{|c|c|c|}
\hline $\begin{array}{l}\text { Forschungsschwerpunkt(e): } \\
\text { Funktionale Grenzen des Strafrechts }\end{array}$ & $\begin{array}{l}\text { Deliktsbereich(e): } \\
\text { Inzest }\end{array}$ & $\begin{array}{l}\text { Rechtsordnung(en): } \\
22 \text { Rechtsordnungen weltweit }\end{array}$ \\
\hline $\begin{array}{l}\text { Zeitrahmen: } \\
\text { 2007-2008 }\end{array}$ & $\begin{array}{l}\text { Status: } \\
\text { laufend }\end{array}$ & $\begin{array}{l}\text { Kategorie: } \\
\text { Institutsprojekt }\end{array}$ \\
\hline
\end{tabular}




\section{Massen- und Kleinkriminalität vor dem italienischen Friedensrichter}

Projektleitung: Dr. Konstanze Jarvers

Zur Bewältigung der Klein- und Massenkriminalität wurden in Italien einige dieser Straftaten in die Zuständigkeit der Friedensgerichte gegeben. Hiermit soll ein alternatives Justizsystem erprobt werden, das durch Milde und Effektivität geprägt ist und zudem das Opfer stärker am Verfahren beteiligt. Die Arbeit kommt zu dem Ergebnis, dass die italienische Reform trotz einiger Schwächen im Detail positiv zu bewerten ist und viele Ansatzpunkte für eigene Reformüberlegungen bietet.

\begin{tabular}{|c|c|c|}
\hline $\begin{array}{l}\text { Forschungsschwerpunkt(e): } \\
\text { Funktionale Grenzen des Strafrechts }\end{array}$ & $\begin{array}{l}\text { Deliktsbereich(e): } \\
\text { Massen- und Kleinkriminalität }\end{array}$ & $\begin{array}{l}\text { Rechtsordnung(en): } \\
\text { Italien }\end{array}$ \\
\hline $\begin{array}{l}\text { Zeitrahmen: } \\
\text { 2004-2007 }\end{array}$ & $\begin{array}{l}\text { Status: } \\
\text { abgeschlossen }\end{array}$ & $\begin{array}{l}\text { Kategorie: } \\
\text { Promotionsprojekt }\end{array}$ \\
\hline
\end{tabular}

\section{Die Rolle der Ehre im Strafrecht}

\section{Projektleitung: Dr. Silvia Tellenbach}

In Zeiten fortschreitender Globalisierung treffen zunehmend Menschen mit unterschiedlichen Ehrvorstellungen zusammen. Daher untersucht das Projekt anhand von 14 Berichten aus ausgewählten Ländern die Bedeutung der Ehre in verschiedenen Aspekten des Strafrechts. Ein Querschnittsbericht zieht ein Fazit. Dabei zeigt sich z.B., dass es deutliche Unterschiede im System der Ehrverletzungstatbestände gibt oder dass Strafmilderungen für Ehrenmorde nur in wenigen Ländern gewährt werden.

\begin{tabular}{|c|c|c|}
\hline $\begin{array}{l}\text { Forschungsschwerpunkt(e): } \\
\text { Funktionale Grenzen des Strafrechts }\end{array}$ & $\begin{array}{l}\text { Deliktsbereich(e): } \\
\text { Straftaten gegen die Ehre }\end{array}$ & $\begin{array}{l}\text { Rechtsordnung(en): } \\
\text { Verschiedene Rechtsordnungen } \\
\text { weltweit }\end{array}$ \\
\hline $\begin{array}{l}\text { Zeitrahmen: } \\
1998^{*}-2007\end{array}$ & $\begin{array}{l}\text { Status: } \\
\text { abgeschlossen }\end{array}$ & $\begin{array}{l}\text { Kategorie: } \\
\text { Institutsprojekt }\end{array}$ \\
\hline
\end{tabular}

\section{Dritter Forschungsschwerpunkt:}

Methodenfragen der Strafrechtsvergleichung und internationale Strafrechtsdogmatik

\section{Internationales Max-Planck-Informationssystem für Strafrechtsvergleichung}

Projektleitung: Prof. Dr. Ulrich Sieber; Dr. Karin Cornils; Phillip W. Brunst

Das Pilotprojekt analysiert an einem komplexen Datenbestand die Methoden der Strafrechtsvergleichung und der Entwicklung einer universalen Strafrechtsdogmatik: Aufgrund einer detaillierten Gliederung werden zwölf Landesberichte zum Allgemeinen Teil des Strafrechts erstellt und vergleichend analysiert. Der dabei entstehende hochstrukturierte Datenbestand dient auch der Entwicklung eines computerbasierten Informationssystems.

\begin{tabular}{|c|c|}
\hline $\begin{array}{l}\text { Forschungsschwerpunkt(e): } \\
\text { Strafrechtsvergleichung }\end{array}$ & $\begin{array}{l}\text { Deliktsbereich(e): } \\
\text { kein spezifischer Deliktsbereich } \\
\text { (Strafrecht Allgemeiner Teil) }\end{array}$ \\
\hline $\begin{array}{l}\text { Zeitrahmen: } \\
\text { 2004-2009 }\end{array}$ & $\begin{array}{l}\text { Status: } \\
\text { laufend }\end{array}$ \\
\hline
\end{tabular}

Rechtsordnung(en):

12 Rechtsordnungen weltweit 


\section{Strafrechtlicher Strukturvergleich}

Projektleitung: Prof. Dr. Walter Perron (Universität Freiburg)

In dem Projekt werden verschiedene Rechtsordnungen des materiellen Strafrechts im Hinblick auf Übereinstimmungen und Abweichungen unter besonderer Berücksichtigung des spezifischen Zusammenspiels von normativer Regelung und praktischer Anwendung erforscht. Zunächst wurden zu dem Thema des Haustyrannenmordes Fallgruppen gebildet, die daraufhin zu untersuchen waren, nach welchen Kategorien sie erfasst werden und wie sie in der Praxis das Strafverfolgungssystem durchlaufen.

Forschungsschwerpunkt(e):

Deliktsbereich(e):

Rechtsordnung(en):

Funktionale Grenzen des Strafrechts;

Tötungsdelikte

8 europäische Rechtsordnungen

Strafrechtsvergleichung

Zeitrahmen:

$1995^{*}-2008$

Institutsprojekt

\section{Strafbare Mitwirkung von Führungspersonen in Straftätergruppen und Netzwerken} in einem lateinamerikanischen Modellstrafgesetz

Projektleitung: Prof. Dr. Ulrich Sieber; Jan-Michael Simon; Dr. Pablo Galain Palermo

Das Projekt analysiert am Beispiel von Gruppenkriminalität, ob ein lateinamerikanisches Zurechnungsmodell von Tatbeiträgen komplexer Kriminalität existiert. Ziel ist es, die Grundlagen für ein lateinamerikanisches Modellstrafgesetzbuch zu legen, was bereits in den 1970er und 1980er Jahren versucht wurde. Zu diesem Zweck werden repräsentativ neun Länder aus der Region rechtsvergleichend untersucht.

Forschungsschwerpunkt(e): Territoriale Grenzen des Strafrechts

Zeitrahmen:

2006-2009
Deliktsbereich(e):

Organisierte Kriminalität; Völkerstraftaten

Status:

laufend
Rechtsordnung(en): 9 lateinamerikanische Länder

Kategorie:

Institutsprojekt 


\section{Forschungsprogramm und Projekte der kriminologischen Abteilung}

\section{FORSCHUNGSPROGRAMM}

Das Forschungsprogramm der kriminologischen Abteilung konzentriert sich auf die Veränderungsprozesse, die im Zuge grundlegender gesellschaftlicher Umbrüche und Transformationen der Gegenwart nicht nur die Entstehungsbedingungen und Gelegenheitsstrukturen von Kriminalität - und damit auch diese selbst - verändern, sondern auch die gesellschaftlichen Reaktionen, die Instrumentarien der formalen Sozialkontrolle und dabei insbesondere der strafrechtlichen Intervention. Dieser inhaltliche Fokus bestimmt den Zuschnitt und die Ausgestaltung der einzelnen Forschungsschwerpunkte. In methodischer Hinsicht werden Schwerpunkte auf die Längsschnittforschung gelegt, die vor allem in der Freiburger Kohortenuntersuchung sowie in der Untersuchung der Wirkungen der Sozialtherapie auf die Rückfälligkeit von Sexualstraftätern zum Ausdruck kommt, sowie auf Mehrebenenanalysen, die die Mikroebene des Handelns mit Makroebenen verknüpfen. Ergänzt wird das Forschungsprogramm daneben durch eine Reihe von internationalen Kooperationsprojekten, die bereits seit langem ein Kernbestandteil der kriminologischen Forschung am Institut sind. Hierzu zählt neben der projektbezogenen Zusammenarbeit im Rahmen der thematisch ausgerichteten Forschungsschwerpunkte insbesondere das seit nunmehr zehn Jahren existierende Laboratoire Européen Associé, das inhaltlich offen angelegt ist.

\section{Schwerpunkt: Strafverfahren und Sanktionen im Wandel}

In dem Gesamtsystem strafrechtlicher Sozialkontrolle stehen das Strafverfahrensrecht wie auch das Sanktionenrecht exemplarisch für die Definition, die Ausgestaltung, die Reichweite und die Grenzen strafrechtlicher Intervention. Gesellschaftliche Transformationen, technologische Entwicklungen und damit einhergehende Veränderungen in den Kriminalitätsphänomenen (insbesondere der sogenannten Transaktionskriminalität, die heute neben den „klassischen“ Bereichen wie der Korruption und dem Vertrieb von Drogen und anderen verbotenen Gütern und Substanzen auch den virtuellen Bereich verbotener Inhalte und anderer Aktivitäten im Cyberspace umfasst) stellen die bestehenden gesetzlichen Regelungen vor neue rechtliche und kriminalpolitische Herausforderungen. Die Weiterentwicklung der bestehenden Eingriffsbefugnisse und die damit verbundenen rechtlichen und praktischen Konsequenzen für das System als Ganzes werden in diesen Bereichen besonders augenfällig, und zwar weit mehr als in anderen Bereichen des (materiellen) Strafrechts. Das kriminologische Forschungsprogramm des Instituts setzt daher einen besonderen Schwerpunkt bei der empirischen Strafverfahrens- sowie der Sanktionsforschung.
In dem Bereich der Strafverfahrensforschung liegt der Arbeitsschwerpunkt bereits seit einigen Jahren auf der Entwicklung, Implementation und Evaluation neuer Technologien im Ermittlungsverfahren. Exemplarisch hierfür steht die Überwachung der Kommunikation. Die kriminalpolitischen Herausforderungen in diesem Bereich lassen sich insbesondere durch das Spannungsverhältnis zwischen der besonderen Grundrechtsrelevanz dieser Eingriffe einerseits (die sich in Risiken für den Kern der Privatsphäre und in der weiten Erfassung nicht beschuldigter Kommunikationsteilnehmer manifestiert) sowie dem Bedürfnis der Strafverfolgungsbehörden nach effektiven Ermittlungsansätzen andererseits charakterisieren. Dieses Bedürfnis ist unmittelbare Folge eines von Ermittlerseite reklamierten strukturellen Defizits, das als zunehmendes Versagen der „klassischen“ strafprozessualen Ermittlungsmethoden infolge des im Bereich der Transaktionskriminalität und anderer Formen der Kontrollkriminalität regelmäBigen Fehlens von Anzeigeerstattern beschrieben wird. Die neuen Ermittlungsmethoden sind zugleich an der Schnittstelle zur Prävention angesiedelt und weisen häufig sogar einen expliziten repressiv-präventiven Doppelcharakter auf. Dieser unmittelbare Bezug zur Prävention verweist 
auf eine weitere Entwicklungslinie aktueller Kriminalpolitik. Insbesondere dort, wo organisierte Kriminalität und Terrorismus im Zentrum stehen, geht es um mehr als die bloße Anpassung der bisherigen Ermittlungsinstrumente auf die im Vergleich zur konventionellen Kriminalität veränderten Einsatzbedingungen. Vielmehr geht es um die gezielte Erweiterung verdeckter Ermittlungsmöglichkeiten. Dies beschreibt auch die eigentliche Kernproblematik der so kontrovers diskutierten Online-Durchsuchung oder der bereits vereinzelt geforderten heimlichen Hausdurchsuchung. Deren Einführung würde eine weitere signifikante Verschiebung in dem Gesamtgefüge der strafprozessualen Eingriffsbefugnisse zur Folge haben und die Entwicklung von den offenen zu den verdeckten Maßnahmen wohl weiter beschleunigen.

Nach dem Abschluss mehrerer Forschungsprojekte zur Implementation und Evaluation der Telekommunikationsüberwachung (2001 bis 2004), zur akustischen Wohnraumüberwachung (2002 bis 2004) sowie zur Rasterfahndung (2005 bis 2007) konzentrierte sich die Arbeit in diesem Bereich zuletzt auf die Studie zur Verwertung von Telekommunikations-Verbindungsdaten (Verkehrsdaten). In dieser Untersuchung wird auch die Interaktion zwischen Privaten (Telekommunikationsprovider) und den Ermittlungs- und Strafverfolgungsbehörden thematisiert (2006 bis 2008). Eine unmittelbar nach Projektabschluss beginnende Replikationsstudie soll die jüngste Veränderung der rechtlichen Rahmenbedingungen unmittelbar aufgreifen und mögliche Veränderungen in der Nutzung von Verkehrsdaten vor dem Hintergrund der Vorratsdatenspeicherung erforschen, die nunmehr auch diejenigen Datenbestände erfasst, die bislang aus Datenschutzgründen bzw. allgemeinen Verhältnismäßigkeitserwägungen nicht oder nur sehr kurzfristig fixiert wurden (2008 bis 2010).

Abgeschlossen wurden im Berichtszeitraum zwei Projekte zur Einstellungspraxis in Fällen leichter Drogen (Cannabis). Die Untersuchungen analysieren die Einstellungspraxis in Deutschland auf der Grundlage von §31a BtMG (2002 bis 2005) sowie vergleichend im Kontext einer breit angelegten internationalen Analyse zu Regelungen des Umgangs mit geringen Mengen von Cannabis zum Eigengebrauch in Kooperation mit der RAND Corporation (2002 bis 2006).

Neu begonnen wurde eine Untersuchung zur Praxis der Durchsuchung von Wohn- und Geschäfts- räumen (2007 bis 2009). Sie wird die Möglichkeiten und Reichweite dieser traditionellen, auf den physischen und nicht-heimlichen Zugriff ausgerichteten Maßnahme speziell auch im Hinblick auf digital gespeicherte Informationen evaluieren. Die Untersuchung soll damit auch einen empirisch fundierten Beitrag zu der aktuellen kriminalpolitischen Debatte um die Notwendigkeit neuer Regelungen auch im Bereich der (Online-) Durchsuchung leisten. Ein weiterer Schwerpunkt der Auswertung wird der Funktion des Richtervorbehalts gewidmet sein. Die Studie wird ergänzt durch eine weitere Untersuchung von Verfahren, die Durchsuchungen in Redaktionsräumen zum Gegenstand haben (2007 bis 2009).

Die empirische Sanktionsforschung repräsentiert ebenfalls einen seit Langem etablierten Forschungsgegenstand des Instituts, der in früheren Jahren unter dem Schwerpunkt „strafrechtliche Sanktionen“ gebündelt war. Im Zentrum stehen Implementations- und Evaluationsstudien zur strafrechtlichen Sanktionierung und zu Fragen des Vollzugs. Abgeschlossen wurde im Berichtszeitraum die Untersuchung zur Praxis von Jugend- und Freiheitsstrafen im Falle schwerer Straftaten, die Parallelen bzw. Unterschiede in der Begründung von Jugend- bzw. (allg.) Freiheitsstrafen im Gruppenvergleich von Jugendlichen, Heranwachsenden und jungen Erwachsenen bei schwerer Kriminalität analysiert hat (2004 bis 2007). Fertig gestellt wurde ferner die Untersuchung zur Berücksichtigung von Angehörigen bei der Verhängung und Aussetzung freiheitsentziehender Sanktionen (2002 bis 2007). Der Schwerpunkt der Analyse liegt hier freilich nicht auf der Täterseite, sondern auf der Drittbetroffenheit durch freiheitsentziehende Sanktionen und ihrer Berücksichtigung durch die Praxis, sei es bei der Zumessung selbst oder bei der Ausgestaltung des Vollzugs oder der Entlassung bzw. vorzeitigen Entlassung.

Weiter in Bearbeitung ist die Begleitforschung zur landesweiten Implementation des elektronisch kontrollierten Hausarrests in Hessen (2003 bis 2008), die eine vorausgegangene wissenschaftliche Begleitstudie zum vorbereitenden Modellversuch weiterentwickelt, deren besonderes Augenmerk neben dem Prozess der Implementation und ihrer Rahmenbedingungen auch der Evaluation der Maßnahmen selbst galt (2000 bis 2004). Ebenfalls noch in Bearbeitung ist die Untersuchung zu Karrierestraftätern in der Volksrepublik China. Im Mittelpunkt dieser Studie stehen die Sanktionierung von Karriere- 
straftätern und daraus resultierende Einflüsse auf die Legalbiographien vor dem Hintergrund der in China dominierenden (Langzeit-)Strafpraxis. Sie berührt auch die Frage nach Implikationen einer bestimmten Kriminalpolitik für die Kriminalprävention (Abschluss 2008).

Neu begonnen wurde eine empirische Studie zur Legalbewährung (2007 bis 2009). Ziel des Kooperationsprojektes mit der Universität Göttingen ist

\section{Schwerpunkt: Gefährliche Straftäter}

Der Forschungsschwerpunkt „Gefährliche Straftäter" greift verschiedene Perspektiven eines Verbrechensphänomens auf, das von der Öffentlichkeit mit ungebrochenem Interesse verfolgt, von der „vierten Gewalt“ nach wie vor medienwirksam aufgemacht und vermarktet wird sowie von der Legislative stetig und gerne für die Begründung strafverschärfender Maßnahmen herangezogen wird. Wissenschaft und Praxis stellt der Umgang mit dieser Tätergruppe vor immer neue Herausforderungen. Wer als gefährlich gilt, wird dabei freilich nicht konform definiert. Sexualstraftäter gelten allgemein als gefährlich. Doch in der Fachwelt ist man sich zunehmend darüber einig, dass nicht alle Sexualstraftäter gleichermaßen gefährlich sind. Gelten Gewaltstraftäter nur als gefährlich, wenn sie wiederholt und in einem bestimmten Ausmaß gewalttätig werden? Eine ähnliche Einstufung als besonders gefährlich erfahren im Übrigen auch Personen aus dem terroristischen Umfeld.

Somit steht der Begriff dieses Forschungsschwerpunkts „Gefährliche Straftäter“ für die Bedeutung einer bestimmten Gruppe von Delinquenten, die als besonders bedrohlich wahrgenommen wird. Die Diskussion über den Umgang mit gefährlichen Straftätern steht im Spannungsfeld zwischen Rechtsstaatsprinzip und dem Ruf nach effizienter Herstellung von Sicherheit. Zusammenhänge zwischen der Entwicklung hin zu einem „gefährlichen Straftäter“, tatsächlicher Gefährlichkeit, Rückfallrisiko und strafrechtlichen Reaktionen sind trotz zunehmender Forschungstätigkeit noch unzureichend beleuchtet. Die kriminologische Abteilung kann durch ihre interdisziplinäre Herangehensweise diese Beziehungen entschlüsseln, die zu einem besseren Verständnis dieser als so bedrohlich wahrgenommenen Tätergruppe beitragen. die Erstellung einer Legalbewährungsstatistik, die Rückfälligkeit im Sinne einer erneuten strafrechtlichen Sanktionierung in Abhängigkeit von Delikt, Sanktionsart, Sanktionshöhe, Vorstrafen und soziodemografischen Merkmalen wie Alter und Geschlecht erfassen soll. Auf diese Weise soll ein Beitrag zu der dringend notwendigen Verbesserung der bislang verfügbaren Datenlage zu Rückfall und Legalbewährung in Deutschland geleistet werden.
Nach dem Abschluss der Nachuntersuchung zur Legalbewährung von Sicherungsverwahrten und gefährlichen Straftätern (2002 bis 2007) steht der Schwerpunkt gegenwärtig ganz im Zeichen des vom Sächsischen Staatsministerium der Justiz geförderten Langzeitprojekts zur Sozialtherapie bei Sexualstraftätern im Freistaat Sachsen (2003 bis 2013). Dieses gliedert sich zwischenzeitlich in drei selbständige Teilstudien. Dies sind zunächst die Evaluationsstudie zur Behandlung von Sexualstraftätern in sozialtherapeutischen Anstalten, deren Hauptziele die Darstellung und Analyse der Rückfallkriminalität von Sexualstraftätern sowie der den Rückfall bedingenden bzw. auf ihn Einfluss nehmenden Faktoren sind, sowie die Studie zu jugendlichen Sexualstraftätern, die sich vor allem der Frage widmet, ob eine frühzeitige Intervention Risikopotenziale verringern kann. Neu begonnen wurde in dem Berichtszeitraum ferner eine Untersuchung zu den Lebensverläufen von Sexualstraftätern nach der Entlassung (2007 bis 2009), die auch auf der Basis von Dunkelfelderhebungen durchgeführt wird. Auf der Grundlage eines prospektiven Designs sollen dynamische Risikofaktoren identifiziert und analysiert werden.

Ergänzend zu den Evaluationsstudien, die sich auf den Freistaat Sachsen konzentrieren, werden die Daten der Kohortenstudie (siehe auch Schwerpunkt 4) für den Forschungsschwerpunkt zur Sexualkriminalität nutzbar gemacht. Dabei wurden im Rahmen mehrerer Sonderauswertungen zur Sexualkriminalität im Längsschnitt alle verfügbaren Daten zu Inzidenz, Prävalenz und zum Rückfall bei den Sexualstraftätern aus den beobachteten Geburtskohorten ausgewertet (2005 bis 2007). Neben Fragestellungen der Prognose wurden auch Formen der Tatbegehung vertiefend analysiert. 
3. Schwerpunkt: Innere Sicherheit, Organisierte Krimialität, Terrorismus - gesellschaftliche Wahrnehmungen und Reaktionen

Ein zentraler Ausgangspunkt der Forschungen in diesem Schwerpunkt ist die Überlegung, dass nicht „objektive“ Risiken durch Kriminalität oder Terrorismus, sondern vielmehr die gesellschaftlichen Wahrnehmungen dieser Risiken handlungsleitend dafür sind, welche kriminalpolitischen Folgerungen gezogen und welche Maßnahmen der Gefahrenabwehr getroffen werden. Daher verdient die Frage, wie die Gesellschaft und wie Individuen Bedrohungen durch Kriminalität und Terrorismus wahrnehmen und welche Sicherheits- und Strafbedürfnisse daraus entstehen, besondere Aufmerksamkeit.

Die individuelle Betroffenheit von Kriminalität und die damit im Zusammenhang stehenden Risikowahrnehmungen und Strafbedürfnisse stehen im Mittelpunkt der Auswertungen des European Crime and Safety Surveys (EU-ICS) 2005 in Zusammenarbeit mit europäischen Partnerinstituten. Damit wird die Tradition der survey-basierten Opferforschung am Max-Planck-Institut fortgeführt und um eine europäisch vergleichende Dimension erweitert. Im Rahmen eines europäischchinesischen Kooperationsprojektes untersucht die auf einer Internetbefragung basierende Studie „Todesstrafe und öffentliche Meinung“ die Kriminalitätswahrnehmungen und Strafeinstellungen chinesischer, amerikanischer und deutscher Studierender. Das ebenfalls neu begonnene, vom Bundesministerium für Bildung und Forschung getragene „Verbundprojekt Sicherheitsforschung“ soll helfen, die sozialwissenschaftliche Forschung zu den gesellschaftlichen Dimensionen der Herstellung von Sicherheit durch technologische Innovationen zu bündeln und neu auszurichten.

Abgeschlossen wurde im Berichtszeitraum ein europäisches Kooperationsprojekt, das die Kriminalprävention in Großwohnsiedlungen in Deutschland, England, den Niederlanden, Polen und Ungarn zum Gegenstand hatte (2005 bis 2007). Hier lag der Schwerpunkt auf den Unsicherheitswahrnehmungen der Bewohner. Beendet wurden ferner die internationale Studie zu den Drogenmärkten in Europa, die anfänglich auf Frankfurt und Mailand beschränkt war, später auf Amsterdam und Genua erweitert wurde (1999 bis 2007), das Projekt „Modelling the World Heroin Market: Assessing the Consequences of Changes in Afghanistan Production“ (2002 bis 2007), sowie das Dissertationsprojekt „Terrorismus - Eine kriminologische Studie“ (2004 bis 2007), in dessen Mittelpunkt die Erforschung der Phänomenologie und der Konstruktion des Terrorismus auf der Grundlage einer Sekundäranalyse stand.

\section{Schwerpunkt: Kriminalität, soziale Kontexte und sozialer Wandel}

Studien zu den Bedingungsfaktoren und zur Entwicklung von Jugendkriminalität haben eine lange Tradition am Max-Planck-Institut. Seit den 1970er Jahren wurden wiederholt Dunkelfeldbefragungen von Jugendlichen zur selbstberichteten Delinquenz und zu Opfererfahrungen durchgeführt. Das inzwischen abgeschlossene Projekt „Soziale Probleme und Jugenddelinquenz in sozialökologischer Perspektive" (1998 bis 2005) hat die deutsche Forschung zu den sozialräumlichen Dimensionen von Jugendkriminalität und deren Wahrnehmung und Kontrolle in Wohngebieten vorangetrieben. Dabei wurden neben der Frage nach der Existenz von Kontexteffekten von Stadtviertelbedingungen auf schwere Jugendkriminalität auch die Fragen nach der informellen Sozialkontrolle und polizeilichen Registrierung von Jugendkriminalität sowie nach der interethnischen Verallgemeinerbarkeit von Delinquenzursachen untersucht. Die qualitativ angelegte, im Rahmen eines DFG-Verbundprojektes mit Partnerinstituten der Universität Freiburg in zwei Teilprojekten durchgeführte Studie zur Hasskriminalität untersucht die Auswirkungen von Hafterfahrungen auf fremdenfeindliche jugendliche Gewalttäter (2004 bis 2008). Ein neu begonnenes Projekt ergänzt diese Studie um eine Analyse der Sozialkonstruktion und Verfolgung von Hasskriminalität in Deutschland (seit 2007).

Die Freiburger Kohortenstudie ist als Langzeitprojekt der kriminologischen Grundlagenforschung angelegt und durch ihr besonderes Design einzigartig in Deutschland. Sie befasst sich mit der Entwicklung des kriminellen Verhaltens von Jugendlichen und jungen Erwachsenen und seiner strafrechtlichen Sanktionierung entlang des Lebenslaufes und zwischen Geburtskohorten bzw. Zeitperioden. Sie verknüpft Daten zur polizeilich registrierten Kriminalität mit denen 
zur strafrechtlichen Sanktionierung. Der in dieser Untersuchung vorhandene Datenbestand zu mehreren Geburtskohorten wird für die Analyse vielfältiger, auch rechtspolitisch aktueller Fragestellungen nutzbar gemacht. Auf PKS-Daten basiert hingegen ein Projekt zur Analyse von Struktur und Entwicklung der Kriminalität auf Kreis- und Gemeindeebene, dessen erste Phase kurz vor dem Abschluss steht (2006 bis 2008), das aber weiter fortgeführt werden soll.

Die laufenden Forschungsprojekte zu tödlicher Gewalt in Familien und Partnerschaften sind auf die Untersuchung von Tötungsdelikten mit anschließendem Suizid des Täters (seit 2006) sowie auf sogenannte „Ehrenmorde“ (begonnen 2008) gerichtet. Tötungsdelikte mit anschließendem Suizid des Täters stellen eine komplexe Form der interpersonellen Gewalt dar, die sich überwie- gend in Partnerschaften und Familien ereignet und die in Deutschland bislang weder statistisch erfasst noch systematisch untersucht wurde. Ziel des Projekts ist eine auf amtlichen Quellen und Medienberichten beruhende Vollerhebung dieser Fälle in Deutschland und weiteren europäischen Ländern (Niederlande, Finnland, Spanien, Polen, England/Wales, Schweiz).

Abgeschlossen wurde das Projekt zur Analyse von Deliktsähnlichkeiten auf der Basis von Individualdaten der Freiburger Kohortenstudie (2003 bis 2007). Hier wurde untersucht, welche Kombinationen von Delikten innerhalb von kriminellen Karrieren besonders häufig (bzw. selten) auftreten. Die Ergebnisse bieten einen gut fundierten empirischen Beitrag zur Identifikation bestimmter auf Delinquenzmustern und Delinquenzverläufen basierender Tätertypologien.

\section{Schwerpunkt: Kriminalpolitik und rechtsstaatliche Entwicklung in außereuropäischen und Übergangsgesellschaften}

Zahlreiche Weltregionen sind durch gesellschaftliche Konflikte, Bürgerkriege oder bürgerkriegsähnliche Unruhen und Kriege erschüttert. Damit verbundene Verwerfungen setzten sich bis in die Post-Konfliktphasen hinein fort. Prekär erscheinen darüber hinaus Systeme, die die Standards eines modernen Rechtsstaates oft über Jahrzehnte hinweg nachhaltig und weitreichend ignorieren oder ignoriert haben. Mit dem Forschungsschwerpunkt „Kriminalpolitik und rechtsstaatliche Entwicklung in (außereuropäischen) Übergangsgesellschaften" verknüpft die kriminologische Forschungsabteilung strafrechtliche und kriminologische Forschungsmethoden, um die Umsetzung rechtsstaatlicher Prinzipien in solchen reformbedürftigen Übergangsgesellschaften wesentlich mit zu gestalten bzw. Grundlagen für eine rationale, internationalen Standards standhaltende Kriminalpolitik zu schaffen.

Abgeschlossen wurde im Berichtszeitraum die in Kooperation mit dem Great Britain China Centre (London) und der Chinese Academy for Social Sciences (CASS, Beijing) durchgeführte Studie zur Stärkung der Verteidigung in Verfahren, in denen die Verhängung der Todesstrafe droht (2005 bis 2006). Neu begonnen wurde eine Studie zur Reform der Todesstrafe in China, die in Kooperation mit dem Great Britain China Centre (London), der Beijing Normal University, der Universität Wuhan, der University of Oxford (Centre for Criminology) sowie dem Irish Centre for Human Rights (Galway) und dem Death Penalty Project (London) durchgeführt wird (2007 bis 2010).

\section{Kooperationsprojekte}

Die Mehrzahl der vormals in einem eigenständigen Querschnittsbereich zusammengefassten nationalen und internationalen Kooperationsprojekte ist nunmehr den jeweiligen thematisch Schwerpunkbereichen zugeordnet. Die abgeschlossenen und sich noch in Bearbeitung befindlichen Projekte betreffen die Kriminalprävention in Großwohnsiedlungen, die Implementation der Anti-Folter-Konvention der

Vereinten Nationen in der Volksrepublik China sowie verschiedene Projekte zu Recht, Politik und Praxis der Todesstrafe in China, das Verbundprojekt zu den Auswirkungen von Hafterfahrungen auf fremdenfeindliche Jugendliche, das Kooperationsprojekt zur Vorbereitung einer bundesweiten Rückfallstatistik, die Auswertung der European Crime and Safety Survey 2005, die vergleichende Evaluation der Einstellungspraxis 
in Cannabis-Fällen, die internationalen Studien zu den Drogenmärkten in Europa und zum WeltHeroin-Markt sowie das europäische Projekt zu Homizid-Suizid-Fällen.

Besonderen Stellenwert im kriminologischen Forschungsprogramm hat darüber hinaus das auf einer gesonderten bilateralen Vertragsbasis zwischen der Max-Planck-Gesellschaft und dem französischen Centre National de la Recherche Scientifique (CNRS) basierende, mit eigenen Finanzmitteln ausgestattete Laboratoire Européen Associé (LEA), in dessen Rahmen seit der Gründung im Jahr 1998 bereits eine Vielzahl thematisch ganz unterschiedlich ausgerichteter empirischer Forschungsprojekte durchgeführt wurden. Im Berichtszeitraum wurde eine neue, soziologisch orientierte Studie zur ethnischen Diskriminierung durch die Polizei in Frankreich und Deutschland begonnen (2006 bis voraussichtlich 2009). Das Projekt greift eine Problematik auf, die anlässlich der Unruhen in mehreren französischen Städten im Herbst 2005 besondere öffentliche Beachtung gefunden hat.

\section{PROJEKTÜBERSICHT}

Hans-Jörg Albrecht

Die folgende Aufstellung gibt einen nach den Forschungsschwerpunkten geordneten Überblick über den Inhalt und die organisatorischen Rahmendaten sämtlicher Forschungsprojekte, die in der kriminologischen Abteilung im Berichtszeitraum 2006/2007 in Bearbeitung waren. Von den insgesamt 30 Projekten wurden acht abgeschlossen und ebenso viele neu begonnen. Neben der inhaltlichen Schwerpunktsetzung ist die Vernetzung mit in- und ausländischen Kooperationspartnern ein weiteres Merkmal der kriminologischen Forschung des Instituts. Insgesamt fallen 13 der aktuellen oder abgeschlossenen Vorhaben in die Kategorie der in- und/oder ausländischen Kooperationsprojekte. Ferner werden oder wurden 12 Projekte von den Bearbeiterinnen und Bearbeitern als Dissertationsprojekte durchgeführt; in drei weiteren Fällen werden oder wurden ausgewählte Fragestellungen bzw. einzelne Teilbereiche im Rahmen von Promotionsvorhaben genutzt.

Erster Forschungsschwerpunkt:

Strafverfahren und Sanktionen im Wandel

Die Auskunftserteilung über Telekommunikationsverbindungsdaten nach $\S \S 100 \mathrm{~g}, 100 \mathrm{~h}$ StP0

Projektleitung: Adina Grafe

Im Zentrum der Studie steht die Untersuchung der Nutzung von Telekommunikationsverkehrsdaten für die Strafverfolgung $(\$ \S 100 \mathrm{~g}, 100 \mathrm{~h}$ StPO). Sie knüpft an die Studien zur Überwachung der Telekommunikation und des Wohnraums an. Verfolgt werden Fragestellungen der Antrags- und Anordnungspraxis, der Auskunftserteilung sowie der Effizienz der Maßnahme. Dabei wird die Kombination mit anderen Ermittlungsmethoden sowie die Einbeziehung Privater in die Strafverfolgung besonders berücksichtigt.

\begin{tabular}{l|l|l} 
Zeitrahmen: & Status: & Kategorie: \\
2005-2007 & $\vdots$ abgeschlossen & Promotionsprojekt
\end{tabular}




\section{Die Implementation der Rasterfahndung}

Projektleitung: Dirk Pehl

Das Forschungsprojekt befasst sich mit der Anwendung und Umsetzung der gesetzlichen Regelungen der Rasterfahndung. Im Mittelpunkt der Untersuchung stehen sowohl Implementations- als auch Evaluationsfragestellungen. Grundlage bildet eine Auswertung von staatsanwaltschaftlichen Ermittlungsakten. Diese wird ergänzt durch bundesweit durchgeführte Expertengespräche.

\begin{tabular}{|c|c|c|}
\hline $\begin{array}{l}\text { Zeitrahmen: } \\
\text { 2005-2007 }\end{array}$ & $\begin{array}{l}\text { Status: } \\
\text { abgeschlossen }\end{array}$ & $\begin{array}{l}\text { Kategorie: } \\
\text { Promotionsprojekt }\end{array}$ \\
\hline
\end{tabular}

\section{Die Durchsuchung von Wohn- und Geschäftsräumen}

\section{Projektleitung: Dr. Michael Kilchling}

Die Durchsuchung von Privat- und Geschäftsräumen gehört zu den besonders häufig eingesetzten strafprozessualen Ermittlungsmaßnahmen, über deren Anwendung nur wenig empirisches Wissen existiert. Selbst einfache Häufigkeitszahlen gibt es in Deutschland nicht. Über die klassischen Evaluationsziele hinaus soll auch erforscht werden, welche Veränderungen die Digitalisierung bei der Anwendung dieser traditionellen, primär auf physische Beschlagnahme ausgerichteten Maßnahme mit sich bringt.

$\begin{array}{lll}\text { Zeitrahmen: } & \text { Status: } & \text { Kategorie: } \\ \text { 2007-2009 } & \text { laufend } & \text { Institutsprojekt }\end{array}$

\section{Pressefreiheit im Kontext strafrechtlicher Ermittlungsmaßnahmen}

Projektleitung: Gunther Olt (Rechtsassessor)

Das Projekt ist Teil der empirischen Strafverfahrensforschung der kriminologischen Forschungsabteilung. Gegenstand der Untersuchung ist ein Themenbereich, der seit der „Spiegel“-Affäre der 1960er Jahre regelmäßig in Öffentlichkeit und Justiz eine beachtliche Resonanz erfahren hat. Das Ergebnis der Untersuchung soll eine empirisch gestützte Beurteilung der Qualität des strafprozessualen Schutzes der Pressefreiheit ermöglichen.

$\begin{array}{lll}\text { Zeitrahmen: } & \text { Status: } & \text { Kategorie: } \\ 2007-2009 & \vdots & 1 \\ & \text { laufend } & \text { Promotionsprojekt }\end{array}$

\section{Cannabis Non-Prosecution Policies in Germany}

Projektleitung: Prof. Dr. Letizia Paoli; Carsten Schäfer

The project conducted in cooperation with the Drug Policy Research Center at the RAND Corporation in the United States investigates the non-prosecution policies against criminal drug users throughout the federal States in Germany. By reviewing case law, statistics and realizing expert interviews the study aimed to analyze the non-prosecution of cannabis-related consumption offences in Germany and to evaluate the different state policies upon consumption.

\begin{tabular}{|c|c|}
\hline $\begin{array}{l}\text { Zeitrahmen: } \\
\text { 2000-2006 }\end{array}$ & $\begin{array}{l}\text { Status: } \\
\text { abgeschlossen }\end{array}$ \\
\hline
\end{tabular}


Die Implementation der elektronischen Fußfessel in Hessen

Projektleitung: Daniela Jessen

Die elektronische Fußfessel als Alternative zur Haft fand in Deutschland im Jahr 2000, zunächst als Modellversuch beim Amts- und Landgericht Frankfurt a.M., ihre erste praktische Anwendung. Mittlerweile in allen Gerichtsbezirken Hessens eingeführt, wird die landesweite Implementierung - nach einer bereits abgeschlossenen Untersuchung des Modellversuchs - durch das Max-PlanckInstitut für ausländisches und internationales Strafrecht wissenschaftlich begleitet und evaluiert.

\begin{tabular}{|c|c|c|}
\hline $\begin{array}{l}\text { Zeitrahmen: } \\
\text { 2003-2009 }\end{array}$ & $\begin{array}{l}\text { Status: } \\
\text { laufend }\end{array}$ & $\begin{array}{l}\text { Kategorie: } \\
\text { Promotionsprojekt }\end{array}$ \\
\hline
\end{tabular}

\section{Legalbewährung nach strafrechtlichen Sanktionen}

Projektleitung: Dr. Carina Tetal; Dr. Volker Grundies

In dieser Studie werden mit dem Ziel der Erstellung einer Rückfallstatistik Verurteilungen im Rückfall, in Abhängigkeit von Delikt, Sanktion und soziodemografischen Merkmalen, wie Alter und Geschlecht, untersucht. Dazu werden alle im Jahr 2004 strafrechtlich Sanktionierten oder aus der Haft Entlassenen über einen dreijährigen Rückfallzeitraum hinweg anhand von Eintragungen im Bundeszentralregister auf erneute Straffälligkeit hin überprüft.

\begin{tabular}{|c|c|}
\hline $\begin{array}{l}\text { Zeitrahmen: } \\
\text { 2007-2009 }\end{array}$ & $\begin{array}{l}\text { Status: } \\
\text { laufend }\end{array}$ \\
\hline
\end{tabular}

\section{Jugendstrafe aufgrund schwerer Kriminalität}

Projektleitung: Benjamin Kurzberg

Vor dem Hintergrund der seit Jahren immer wieder aufflammenden Diskussion über eine Verschärfung der Sanktionspraxis gegenüber heranwachsenden Delinquenten verfolgt die Arbeit vor allem zwei Fragestellungen: Wie lässt sich die Verhängung von langen Haftstrafen mit dem im Jugendstrafrecht vorherrschenden Erziehungsgedanken dogmatisch vereinbaren? Und: Wie wird in der aktuellen Justizpraxis auf schwere Kriminalität junger Täter reagiert?

$\begin{array}{lll}\text { Zeitrahmen: } & \text { Status: } & \text { Kategorie: } \\ \text { 2005-2007 } & \text { abgeschlossen } & \vdots\end{array}$

\section{Kriminelle Karrieren und Kriminalprävention}

Projektleitung: Fan Wen

Die Untersuchung greift Fragen der Karrieretäterforschung im Bezug auf die ökonomische Transformation und den gesellschaftlichen Wandel in China auf. Ausgewertet werden Daten, die in China erhoben wurden. Analysiert wird zum einen, ob und wie sich die strafrechtliche Sanktionierung - hier in erster Linie die in China dominierenden Freiheitsstrafen - auf den Verlauf von kriminellen Karrieren auswirkt, zum anderen, wie unter diesen Bedingungen sozial-psychologische Merkmale wie Schichtzugehörigkeit, Bildung oder das familiäre Umfeld die Ausbildung krimineller Karrieren beeinflussen. 
Zweiter Forschungsschwerpunkt:

Gefährliche Straftäter

\section{Sexualstraftäter als Herausforderung für Forschung und Kriminalpolitik}

Projektleitung: Dipl.-Psych. Dr. Gunda Wößner

Kernstück dieses prospektiv angelegten Projektes ist die Evaluation der sozialtherapeutischen Behandlung von Sexual- und Gewaltstraftätern und die Identifizierung rückfallrelevanter Einflussfaktoren. Hierfür werden im Regelvollzug bzw. in der Sozialtherapie Sachsens inhaftierte Sexual- und Gewaltstraftäter exploriert. Neben einer kontinuierlichen und erweiterten Datenerhebung wurde im Berichtszeitraum an der Auswertung erster Zwischenergebnisse und Realisierung diverser Teilprojekte gearbeitet.

\begin{tabular}{llll} 
Zeitrahmen: & $\begin{array}{l}\text { Kategorie: } \\
2003-2013\end{array}$ & $\begin{array}{l}\text { Status: } \\
\text { Institutsprojekt }\end{array}$ \\
\hline
\end{tabular}

\section{Jugendliche Sexualstraftäter in sozialtherapeutischen Anstalten des Freistaates Sachsen}

Projektleitung: Dipl.-Psych. Dr. Gunda Wößner

Im Fokus dieser Studie steht die Frage, welche Maßnahmen bei jugendlichen Sexualstraftätern geeignet sind, Rückfall zu verhindern. Damit zusammenhängend wird untersucht, ob und in welchen Merkmalen sich jugendliche Sexualstraftäter von erwachsenen unterscheiden bzw. inwiefern spezifische rückfallrelevante Faktoren wirksam werden. Das Design der Studie entspricht dem Evaluationsprojekt zu erwachsenen Sexualstraftätern. Bisher wurden ca. 60 jugendliche Gefangene befragt.

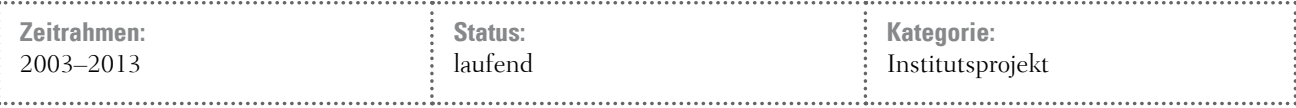

\section{Lebensverläufe von Sexualstraftätern nach Entlassung}

Projektleitung: Dipl.-Psych. Sonja Brauner

Die Studie ist Teil des Projektes „Sexualstraftäter in sozialtherapeutischen Anstalten des Freistaates Sachsen“. Ziel ist es, Risikofaktoren des Rückfalls in den Lebensumständen von Sexualstraftätern nach Entlassung aus der Sozialtherapie zu identifizieren und damit Lücken in der Rückfallforschung zu schließen. Zusätzlich werden Dunkelfelddaten erhoben, die Auskunft über Ausmaß und Anlässe von Rückfalltaten im Nachentlasszeitraum geben.

\begin{tabular}{|c|c|c|}
\hline $\begin{array}{l}\text { Zeitrahmen: } \\
\text { 2007-2009 }\end{array}$ & $\begin{array}{l}\text { Status: } \\
\text { in Planung }\end{array}$ & $\begin{array}{l}\text { Kategorie: } \\
\text { Promotionprojekt }\end{array}$ \\
\hline
\end{tabular}




\section{Jugendliche und heranwachsende Sexualstraftäter}

Projektleitung: Dipl.-Psych. Carolin Quenzer

Das Projekt fokussiert junge Sexualstraftäter. Die zentrale Fragestellung befasst sich mit der Vorhersagbarkeit von Rückfälligkeit bei dieser Tätergruppe. Das Ziel der Studie ist die Überprüfung der Vorhersagegenauigkeit von verschiedenen, speziell für junge Täter entwickelten Prognoseinstrumenten. Im Rahmen der Untersuchung werden erstmalig im amerikanischen Raum entwickelte Prognoseverfahren bei einer deutschen Stichprobe angewendet.

$\begin{array}{llll}\text { Zeitrahmen: } & \text { Status: } & \text { Kategorie: } \\ 2006-2009 & \text { laufend } & \text { Promotionsprojekt }\end{array}$

\section{Sexualkriminalität im Längsschnitt}

\section{Projektleitung: Dr. Volker Grundies}

Die Daten der Freiburger Kohortenstudie, die nunmehr sechs Geburtskohorten aus Baden-Württemberg sowie die für diese registrierten polizeilichen und justiziell dokumentierten Straftaten umfasst, werden im Hinblick auf Inzidenz und Prävalenz von Sexualstraftaten analysiert. Während im vorangehenden Berichtszeitraum v.a. Fragen der Prognose von Rückfällen auf der Basis der Legalbiographie untersucht wurden, wurde in diesem Berichtszeitraum ergänzend Formen der Tatbegehung deskriptiv analysiert.

$\begin{array}{lll}\text { Zeitrahmen: } & \begin{array}{l}\text { Status: } \\ \text { laufend }\end{array} & \vdots \\ 2005-2008 & \text { lategorie: } & \\ \text { Institutsprojekt }\end{array}$

\section{Die Legalbewährung gefährlicher Rückfalltäter}

Projektleitung: Prof. Dr. Jörg Kinzig

Im Anschluss an die im Jahr 1996 veröffentlichte Studie „Die Sicherungsverwahrung auf dem Prüfstand" wurden rund zehn Jahre danach die BZR-Auszüge der damals rund 500 untersuchten Probanden einer Sicherungsverwahrungs- und einer Kontrollgruppe eingeholt und ausgewertet (insbesondere nach dem Vollstreckungsverlauf und einer etwaigen Legalbewährung). Eingebettet ist diese Analyse in eine Beschreibung der aktuellen Entwicklung des Rechts der Sicherungsverwahrung.

\begin{tabular}{l|ll} 
Zeitrahmen: & $\begin{array}{l}\text { Status: } \\
\text { 1999-2007 }\end{array}$ & Kategorie: \\
& $\vdots$ abgeschlossen & $\vdots$
\end{tabular}


Dritter Forschungsschwerpunkt:

Innere Sicherheit, organisierte Kriminalität, Terrorismus

- gesellschaftliche Wahrnehmung

\section{Drug Markets in Europe}

\section{Projektleitung: Prof. Dr. Letizia Paoli}

The project, funded by the EMCDDA, aims to fill a gap in European drug research by analysing the illegal drug markets of four major European cities. In contrast to a few already realized studies, which concentrate on consumption patterns, this investigation has its focus on the interplay of drug demand and supply. Moreover it compares the drug markets of the four cities in their entirety. The results of the four case studies will be published in an edited book in the course of 2008 .

$\begin{array}{llll}\text { Zeitrahmen: } & \text { Status: } & \text { Kategorie: } \\ \text { 1999-2007 } & \text { abgeschlossen } & \text { Institutsprojekt }\end{array}$

\section{Modelling the World Heroin Market: Assessing the Consequences of Changes in Afghanistan Production}

Projektleitung: Prof. Dr. Letizia Paoli; Prof. Peter Reuter (University of Maryland and RAND Corporation); Prof. Dr. Victoria Greenfield (Naval Academy)

The project was funded by the Netherlands Ministry of Justice, the Smith Richardon Foundation and the UK Foreign and Commonwealth Office and realized by the Drug Policy Research Center and the RAND Corporation and the MPI. It aimed to clarify the coherences in heroin production, prices and flows to facilitate intervention measures. It also analysed the consequences of interventions in Afghanistan drawing from the cutback in opium production achieved by the Islamist Taliban regime in 2001 .

\begin{tabular}{l|ll} 
Zeitrahmen: & Status: & Kategorie: \\
2002-2007 & abgeschlossen & Institutsprojekt
\end{tabular}

\section{Auswertungen mit dem European Crime and Victim Survey 2005}

Projektleitung: Dr. Dina Hummelsheim; Priv.-Doz. Dr. Dietrich Oberwittler

Der European Crime and Safety Survey wurde als Teil der fünften Welle des International Crime Victim Surveys 2004/2005 erhoben. Es handelt sich hierbei um eine repräsentative Bevölkerungsbefragung in 18 europäischen Ländern. Auf der Basis dieser Umfragedaten widmet sich das Forschungsprojekt der empirischen Untersuchung von Länderunterschieden in Viktimisierungserfahrungen und kriminalitätsbezogenen Einstellungen.

$\begin{array}{llll}\text { Zeitrahmen: } & \text { Status: } & \text { Kategorie: } \\ 2008-2010 & \text { in Planung } & \text { Institutsprojekt }\end{array}$




\section{Regionalanalysen der registrierten Kriminalität}

Projektleitung: Priv.-Doz. Dr. Dietrich Oberwittler

Gegenstand des Projekts ist die Untersuchung der räumlichen Strukturen der registrierten Kriminalität im Quer- und Längsschnitt mit dem Schwerpunkt Baden-Württemberg. Das Ziel ist eine differenzierte und theoriegeleitete Analyse und Erklärung der Kriminalitätsverteilungen auf der Ebene von Gemeinden und Kreisen auf der Basis der polizeilich registrierten Kriminalität und einer Reihe amtlicher Strukturdaten einschließlich geographischer Merkmale.

\begin{tabular}{|c|c|c|}
\hline $\begin{array}{l}\text { Zeitrahmen: } \\
\text { 2007-2009 }\end{array}$ & $\begin{array}{l}\text { Status: } \\
\text { laufend }\end{array}$ & $\begin{array}{l}\text { Kategorie: } \\
\text { Institutsprojekt }\end{array}$ \\
\hline
\end{tabular}

\section{Crime Prevention Carousel}

Projektleitung: Dipl.-Soz. Tim Lukas

Are attempts to rehabilitate high-rise housing estates an effective way to reduce crime and feelings of insecurity in areas of this nature? This is the pivotal question of the Crime Prevention Carousel, an international comparative study co-ordinated by the Max Planck Institute. Focusing on both situational and social approaches to crime prevention the study aims to share experiences about how best to reduce neighbourhood crime and feelings of insecurity in high-rise residential housing estates.

\begin{tabular}{l|ll} 
Zeitrahmen: & $\vdots$ Status: & Kategorie: \\
2005-2008 & $\vdots$ laufend & $\vdots$
\end{tabular}

\section{Vierter Forschungsschwerpunkt:}

Kriminalität, soziale Kontexte und sozialer Wandel

\section{Familiale Tötungsdelikte mit anschließendem Suizid in europäischen Ländern}

Projektleitung: Priv.-Doz. Dr. Dietrich Oberwittler

Tötungsdelikte mit anschließendem Suizid des Täters stellen eine sehr schwere Form der Gewalt im sozialen Nahraum dar. Ziel des Projekts ist eine auf amtlichen Quellen und Medienberichten beruhende Vollerhebung dieser Fälle in Deutschland und in weiteren Ländern in den Jahren 19962005. Die Analyse auf der Basis einer Zufallsstichprobe von ca. 350 Akten und ca. 40 Interviews mit überlebenden Tätern ist interdisziplinär und auf mehreren Ebenen angelegt. 


\section{Ehrenmorde in Deutschland}

Projektleitung: Priv.-Doz. Dr. Dietrich Oberwittler

Das Projekt verfolgt das Ziel einer Bestandsaufnahme der bekannt gewordenen Fälle von Ehrenmorden in Deutschland im Zeitraum 1996 bis 2005 auf der Basis staatsanwaltschaftlicher Akten und von Medienberichten. In der empirischen Analyse sollen Täter-Opfer-Konstellation, Tathergang, Anlass/Motiv sowie justizielle Verarbeitung systematisch untersucht und damit Aussagen über die Bedeutung dieses Phänomens in Deutschland ermöglicht werden.

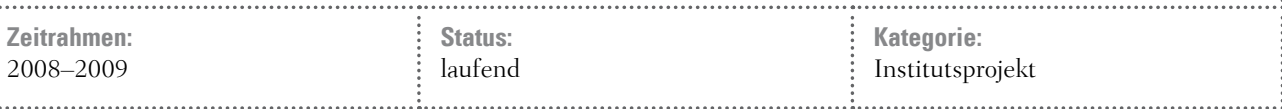

\section{Sozialkonstruktion und Verfolgung von Hasskriminalität}

Projektleitung: Alke Glet, M.A. Internationale Kriminologie

Das Themenfeld „Hasskriminalität“ zeichnet sich in Deutschland durch Abgrenzungsschwierigkeiten in der Begrifflichkeit und empirische Defizite aus. Die Untersuchung soll daher nicht nur Aufschluss über die Prävalenz hassmotivierter Straftaten geben, sondern auch ein Grundverständnis dieses Phänomens in seinem sozialen Kontext schaffen, indem die relevanten Akteure im Hinblick auf die Definition, Erfassung, Strafverfolgung und öffentliche Darstellung von Hasskriminalität näher beleuchtet werden.

\begin{tabular}{l|l|l} 
Zeitrahmen: & Status: & Kategorie: \\
$2007-2010$ & laufend & Promotionsprojekt
\end{tabular}

\section{Ethnische Diskriminierungen durch die Polizei in Frankreich und Deutschland}

Projektleitung: Jérémie Gauthier

Das LEA-Projekt greift die Unruhen in den französischen Städten 2005 auf und untersucht die soziale, ethnische und geographische Deklassierung junger Bewohner aus den Vorstädten größerer und mittlerer französischer Agglomerationen. Im Vergleich mit Deutschland, wo es entsprechende Ausschreitungen bislang nicht gab, können Unterschiede zwischen der deutschen und französischen Polizei und ihrem Verhältnis zur Bevölkerung, insbesondere zu den Gruppen mit Migrationshintergrund, gemessen werden.

\begin{tabular}{l|l|l|} 
Zeitrahmen: & Status: & Kategorie: \\
2006-2009 & $\vdots$ laufend & $\vdots$ Institutsprojekt
\end{tabular}

\section{Hasskriminalität}

Projektleitung: Prof. Dr. Hans-Jörg Albrecht

Das Projekt ist Teil eines von der Deutschen Forschungsgemeinschaft (DFG) geförderten Verbundprojekts „Recht, Norm, Kriminalisierung“ mit dem Anglistischen Seminar und dem Husserl-Archiv der Universität Freiburg. Ziel ist es, die Folgen von Jugendhaft auf die Entwicklungsprozesse von rechtsextremistischen Gewalttätern zu untersuchen. Im Zentrum stehen Fragen nach der Veränderung der Identität, den Bindungen an rechtsextremistische Überzeugungen und der Gewaltbereitschaft im Inhaftierungsverlauf.

$\begin{array}{llll}\text { Zeitrahmen: } & \text { Status: } & \text { Kategorie: } \\ \text { 2004-2008 } & \text { laufend } & \text { Institutsprojekt }\end{array}$


Freiburger Kohortenstudie zur Entwicklung polizeilich registrierter Kriminalität und strafrechtlicher Sanktionierung

Projektleitung: Dr. Volker Grundies

Die Freiburger Kohortenstudie ist ein langfristig angelegtes Projekt der kriminologischen Grundlagenforschung, das durch sein besonderes Kohortendesign einzigartig in der Bundesrepublik ist. Anhand polizeilicher und justizieller Daten wird die Kriminalitätsentwicklung sowohl altersspezifisch als auch als Reaktion auf gesamtgesellschaftliche Entwicklungen über die Zeit analysiert. Weiter werden die institutionellen Reaktionen auf Delinquenz und die Auswirkung dieser Reaktionen untersucht.

$\begin{array}{llll}\text { Zeitrahmen: } & \text { Status: } & \text { Kategorie: } \\ \text { Beginn } 1985 & \text { laufend } & \vdots\end{array}$

\section{Analyse von Deliktsähnlichkeiten auf der Basis von Individualdaten}

Projektleitung: Dr. Carina Tetal

Um typische Deliktsmuster zu erfassen, wird in diesem Projekt untersucht, welche Kombinationen von Delikten innerhalb von kriminellen Karrieren auftreten. D.h. es werden Analysen zu Spezialisierung in mehrfachem Sinne durchgeführt. Einerseits wird Spezialisierung innerhalb einzelner Deliktskategorien untersucht, andererseits wird der Zusammenhang verschiedener Deliktskategorien mit dem Ziel erforscht, typische Deliktsmuster zu bestimmen, innerhalb derer es auch zu Spezialisierungen kommt.

$\begin{array}{llll}\text { Zeitrahmen: } & \text { Status: } & \text { Kategorie: } \\ 2003-2007 & \text { abgeschlossen } & \text { Promotionsprojekt }\end{array}$

Fünfter Forschungsschwerpunkt:

Kriminalpolitik und rechtsstaatliche Entwicklung

in außereuropäischen und Übergangsgesellschaften

\section{Moving the Debate Forward}

Projektleitung: Prof. Dr. Hans-Jörg Albrecht

Unter Beteiligung des Great Britain China Centre (London) wird ein von der Europäischen Kommission finanziertes Projekt zum Thema „Moving the Debate Forward: China's Use of the Death Penalty" durchgeführt, dessen Ziel es ist, Zusammenhänge zwischen öffentlicher Meinung, Rechtspolitik, Gesetzgebung und Justizpraxis zu untersuchen und Einstellungsänderungen bei Akteuren der Politik und der Justizpraxis sowie in der Öffentlichkeit zu initiieren.

\begin{tabular}{l|ll} 
Zeitrahmen: & Status: & Kategorie: \\
$2007-2010$ & $\vdots$ laufend & Institutsprojekt
\end{tabular}




\section{Strengthening the Defence in Death Penalty Cases in China}

Projektleitung: Prof. Dr. Hans-Jörg Albrecht

The joint comprehensive project entitled „Strengthening the Defence in Death Penalty Cases in China" has several purposes. Besides training of defence lawyers, the project examines the role defence lawyers play in Chinese criminal proceedings and particularly in death penalty proceedings. It aims to point out what can and should be done to empower defence councils to play an effective role in death penalty eligible cases and, more generally, to establish an effective system of legal aid.

$\begin{array}{lll}\text { Zeitrahmen: } & \text { Status: } & \text { Kategorie: } \\ 2005-2006 & \vdots & \text { Institutsprojekt }\end{array}$

\section{Modellgesetzbücher für Post-Konflikt-Gesellschaften}

Projektleitung: Prof. Dr. Hans-Jörg Albrecht

In dem von mehreren internationalen Organisationen initiierten, zusammen mit zahlreichen ausländischen Partnern durchgeführten Projekt werden Modellgesetzbücher für Post-Konflikt-Gesellschaften entwickelt. Die Modellgesetzbücher (ein Strafgesetzbuch, eine Strafprozessordnung, ein Strafvollzugs- und ein Polizeigesetz) werden in kommentierten Versionen veröffentlicht und sollen eine Grundlage für den (Wieder-)Aufbau von Kriminaljustiz und Ordnungsystemen in Post-Konflikt-Gesellschaften bilden.

\begin{tabular}{l|ll} 
Zeitrahmen: & Status: & Kategorie: \\
$2001-2008$ & laufend & Institutsprojekt
\end{tabular}




\section{Aktuelle Forschungsarbeiten}




\section{AKTUELLE FORSCHUNGSARBEITEN}
A. Überblick
B. Transnationale Kriminalität und territoriales Sicherheitsrecht: Nationale Abschottung im globalen Cyberspace?

C. Terrorismusbekämpfung jenseits der funktionalen Grenzen des Strafrechts: Kontrollverfügungen gegen mutmaßliche Terroristen im Vereinigten Königreich

D. Neue informationstechnische Überwachungsmaßnahmen: Online-Durchsuchung und Online-Überwachung vor dem Bundesverfassungsgericht

E. Aktuelles Zeitgeschehen:

Steuerermittlungen in Liechtenstein

F. Internationale Kriminalpolitik:

Strafrechtspolitik in China - Auf dem Weg zur Abschaffung der Todesstrafe?

G. Risiko und Risikokontrolle:

Forschungsschwerpunkt Sexualstraftäter

H. Informationsgesellschaft:

Die Überwachung der Telekommunikation

I. Verbrechensfurcht:

Schützt soziale Sicherheit vor Kriminalitätsfurcht?

J. Strafrecht und Kriminologie unter einem Dach:

Grenzen des Rechtsgüterschutzes vor dem Bundesverfassungsgericht 


\section{A. Überblick}

Die Forschungsprojekte am Max-Planck-Institut für ausländisches und internationales Strafrecht sind in Kapitel I. B. und C. in zwei tabellarischen Aufstellungen genannt. Im Folgenden wird an ausgesuchten Arbeiten ein näherer Einblick in die Forschungstätigkeit des Instituts gegeben, die vor allem umfassende Untersuchungen und Dissertationen beinhaltet, aber auch wichtige - in den obigen Projektdarstellungen meist nicht erfasste - Gutachten und kleinere Publikationen.

Für die strafrechtliche Abteilung hat der letzte Forschungsbericht 2004/05 bereits den Methodenschwerpunkt der Strafrechtsvergleichung mit zwei ausführlichen Beschreibungen zu den Projekten „Max-Planck-Informationssystem für Strafrechtsvergleichung“ und „Strafbare Mitwirkung von Führungspersonen in Straftätergruppen und Netzwerken“ vorgestellt (vgl. Forschungsbericht 2004/05, S. 42-51, 86-92). Der vorliegende Forschungsbericht 2006/07 verdeutlicht deswegen nunmehr die Schwerpunkte zu den territorialen und den funktionalen Grenzen des Strafrechts. Am Anfang steht eine umfassende Untersuchung zu den territorialen Grenzen des Strafrechts am Beispiel des Cyberspace, gefolgt von einer Dissertation zu den funktionalen Grenzen des Strafrechts bei der Verfolgung von Terrorismus in Großbritannien. Dem schließen sich zwei weitere Beiträge zu den Grenzen des Strafrechts an, der erste zu einem informationsrechtlichen Gutachten im Auftrag des Bundesverfassungsgerichts und der zweite zu einem besonders aktuellen Thema aus der Vermischung von strafrechtlicher Ermittlung und geheimdienstlicher Untersuchung.

- Der erste Projektbericht fasst die Ergebnisse der Untersuchung über „Nationale Abschottung im globalen Cyberspace“ zusammen. Er belegt für die Internetkriminalität, dass sich der Nationalstaat gegen transnationale Kriminalität nicht mehr mit rein nationalen Maßnahmen an seinen territorialen Außengrenzen ausreichend schützen kann, sondern transnational wirksame Schutzmechanismen benötigt.

- Der zweite Bericht mit neuen Forschungsergebnissen über die britischen Kontrollverfügungen gegen mutmaßliche Terroristen untersucht Präventionsmaßnahmen jenseits der funktionalen Grenzen des Strafrechts und im Grenzbereich möglicher Menschenrechtsverletzungen. Er zeigt die - für die gegenwärtige Entwicklung typische - Ergänzung und Ersetzung des klassischen (eine bereits begangene Straftat voraussetzenden) repressiven Strafrechts durch ein (auf zukünftige Gefahren zielendes) präventives Sicherheitsrecht, das auf manche Sicherungen des traditionellen liberalen Strafrechts verzichten will.

- Zum gleichen Forschungsschwerpunkt verdeutlicht das Gutachten des Instituts im Verfahren des Bundesverfassungsgerichts zur Online-Durchsuchung die Relevanz der rechtswissenschaftlichen Forschung für einen angemessenen Ausgleich von Sicherheit und Freiheit: Das Bundesverfassungsgericht hat die in dem Gutachten vorgezeichnete vermittelnde Position aufgenommen.

- Der Beitrag zur Erlangung der Liechtensteiner Kontendaten durch die deutschen Steuerbehörden in Zusammenarbeit mit dem Bundesnachrichtendienst zeigt an einem neueren Beispiel die Aktualität des vom Institut im Jahre 2004 gewählten Forschungsprogramms, das die Probleme der transnationalen Ermittlungen und der „Vergeheimdienstlichung“ des Strafverfahrens hervorhebt.

Die weiteren Berichte verdeutlichen die Schwerpunkte des kriminologischen Forschungsprogramms, vor allem zum Wandel des Strafverfahrens, zu gefährlichen Straftätern und zur gesellschaftlichen Wahrnehmung von komplexer Kriminalität: 
- Der Text über die Einstellung der chinesischen Bevölkerung zur Todesstrafe eruiert die Grundlagen für eine Zurückdrängung der Todesstrafe in China und gibt ein Beispiel für die Arbeiten des Instituts im Bereich der internationalen Kriminalpolitik.

- Der Beitrag über die Behandlung gefährlicher Straftäter untersucht für diesen speziellen Personenkreis in der Sache die gleiche Fragestellung wie der Bericht der strafrechtlichen Forschungsabteilung für terroristische „Gefährder“ in Großbritannien: Wie geht die Gesellschaft mit potentiell gefährlichen Personen um, bevor sich das entsprechende Risiko in einer Straftat manifestiert hat?

- Die anschließende Analyse zur Telekommunikationsüberwachung betrifft - ganz ähnlich wie der oben genannte Beitrag aus der strafrechtlichen Forschungsabteilung zur Online-Durchsuchung die intensiver werdenden Eingriffsmaßnahmen der Informations- und Risikogesellschaft, die sich durch Heimlichkeit, aber auch eine großflächige Erfassung von zahlreichen Personen, die Erstreckung in das Vorfeld von Straftaten sowie intensive Eingriffe in die Privat- und Intimsphäre auszeichnen.

- Die abschließende Untersuchung über den Zusammenhang zwischen sozialer Sicherheit und Kriminalitätsfurcht ist vor allem auch für die von beiden Forschungsabteilungen gemeinsam verfolgten rechtspolitischen Zielsetzungen von Bedeutung, da die Verschärfung des Strafrechts in der modernen Risikogesellschaft nach den in beiden Forschungsabteilungen zugrunde gelegten Hypothesen nicht nur durch objektive Risikosteigerungen verursacht wird, sondern auch durch subjektive Einstellungen wie Kriminalitätsfurcht.

Der letzte Bericht über die Grenzen des Rechtsgüterschutzes beruht auf einem Gutachtenauftrag des Bundesverfassungsgerichts in dem von der Presse intensiv diskutierten Verfahren zur Inzeststrafbarkeit. Er beschreibt die Ergebnisse eines Gemeinschaftsprojekts der strafrechtlichen und der kriminologischen Forschungsabteilung. Die Anfrage des Bundesverfassungsgerichts nach kriminologischen und rechtsvergleichenden Erkenntnissen bestätigt dabei den vom Institutsgründer Hans-Heinrich Jescheck und seinem Kollegen Günther Kaiser begründeten Forschungsansatz von „Strafrecht und Kriminologie unter einem Dach“, der am Freiburger Institut nicht nur durch solche Gemeinschaftsprojekte, sondern auch durch die weitgehend deckungsgleichen Forschungsziele der beiden Institutsabteilungen verwirklicht wird. 


\title{
B. Transnationale Kriminalität und territoriales Sicherheitsrecht
}

\author{
Nationale Abschottung im globalen Cyberspace
}

Das Internet ermöglicht einen weltweiten Zugriff auf eine Vielzahl von rechtmäßig angebotenen Daten. Es wird aber auch für die Begehung von Straftaten genutzt, etwa zur Verbreitung von gewaltverherrlichenden oder kinderpornographischen Inhalten sowie für Urheberrechtsverletzungen, illegales Glücksspiel oder die Werbung für terroristische Ziele.

Eine Verhinderung und Verfolgung dieser Delikte bereitet häufig Schwierigkeiten, da die Kompetenzen der nationalen Sicherheitsbehörden an den Staatsgrenzen enden. Auch wenn der Anwendungsbereich des materiellen Strafrechts auf ausländische Server ausgedehnt wird, ist die internationale Rechtsdurchsetzung wegen der fehlenden Gebietshoheit in der Praxis schwierig. Die Nationalstaaten können deswegen in vielen Fällen nicht wirksam gegen illegale Inhalte auf ausländischen Servern vorgehen. Das gilt vor allem dann, wenn diese Inhalte im Ausland nicht strafbar sind, wie es etwa in den USA bei nationalsozialistischer Propaganda oder vielen gewaltverherrlichenden Äußerungen der Fall ist.

Viele Staaten versuchen daher, ihr nationales Territorium gegen illegale Inhalte im Internet abzuschotten: Wenn nicht direkt gegen die auf ausländischen Servern gespeicherten Inhalte vorgegangen werden kann, so sollen wenigstens technische Sperrmaßnahmen einen Zugriff der Bürger unterbinden. Bekannt sind die Maßnahmen der Volksrepublik China oder des Iran, welche die Informations- und Meinungsfreiheit stark beschränken. Seit einigen Jahren implementieren jedoch auch westliche Demokratien wie Großbritannien zunehmend technische Sperren gegen illegale Inhalte im Internet. Auch in Deutschland bestehen bereits gesetzliche Grundlagen für die Anordnung derartiger Zugangssperren durch die Medienaufsicht. Diese „Sperrverfügungen“ waren in Deutschland bisher allerdings in der praktischen Umsetzung wenig erfolgreich und wurden in der (Fach-)Öffentlichkeit wegen ihrer technischen Probleme und ihrer Eingriffe in die Informations- und Meinungsfreiheit überwiegend kritisch beurteilt.

Wegen der bestehenden technischen und rechtlichen Unsicherheiten im Hinblick auf Sperrverfügungen im Internet beauftragte die in Deutschland für Sperrverfügungen zuständige Kommission für Jugendmedienschutz (KJM) das Freiburger Max-Planck-Institut für ausländisches und internationales Strafrecht sowie die Technische Universität Dresden mit zwei Studien zur Klärung dieser Problematik. Ziel war zum einen die Untersuchung der technischen Möglichkeiten, zum anderen die Prüfung der rechtlichen Zulässigkeit von Sperrmaßnahmen im Internet. Das Max-Planck-Institut konnte sich deswegen in der zweitgenannten Studie für die Beantwortung der Fragestellung in methodischer Hinsicht nicht nur auf seine eigene rechtliche und technische Analyse stützen, sondern auch auf das speziell zur Beantwortung der Rechtsfragen erstellte informationstechnische Gutachten der Technischen Universität Dresden. Die vom Max-Planck-Institut durchgeführte juristische Analyse führte dabei zu unerwarteten Ergebnissen.

\section{Technische Möglichkeiten der territorialen Zugriffsbeschränkung im globalen Internet}

In der Diskussion über die Grundstrukturen von internationalen Computernetzen dominiert heute noch immer das Paradigma des national kaum regulierbaren „globalen Cyberspace“. Gerade frühe Visionäre des Informationsrechts stellen inzwischen jedoch die Frage nach einer „Territorialisierung" des Internets, um den Nationalstaaten auch online einen Restbestand ihrer Souveränität zu erhalten und die Durchsetzung von Normen und Werten nicht allein dem Markt zu überlassen.

Entsprechende Möglichkeiten zeigen sich gerade im Bereich der Sperrmaßnahmen gegen bestimmte Inhalte: Seitdem diese vor einigen Jahren in Deutsch-
Strafrechtliche Forschungsabteilung

Forschungsschwerpunkt: Territoriale Grenzen des Strafrechts

Deliktsbereich: Cybercrime 
land erstmals gerichtlich überprüft wurden, hat sich die Technik von Internetsperren erheblich weiterentwickelt. Die Umsetzungsmöglichkeiten hängen in erster Linie vom Diensteangebot der Provider ab, die zur Einrichtung von Zugriffssperren herangezogen werden. Denkbare Akteure für Sperrmaßnahmen sind Access-Provider, die den Einwahlknoten zum Internet bereitstellen, und Network-Provider, die die Infrastruktur betreiben.

Entscheidend für die Durchführung von Sperrmaßnahmen sind dabei die Daten, die den jeweiligen Anbietern bei ihrer Geschäftserbringung vorliegen. Stark vereinfacht lässt sich feststellen, dass drei Ansätze zur Verfügung stehen: „Grobe" Sperrtechniken (wie solche anhand der IPAdresse) sind einfach durchzuführen, aber nicht trennscharf, da sie oft zahlreiche legale Inhalte miterfassen, die nur zufällig am gleichen Ort gespeichert sind. „Feinere“ Sperrtechniken (insbesondere auf der Basis von URL-Adressen) sind zwar von größerer Treffsicherheit, setzen jedoch einen hohen technischen und organisatorischen Aufwand voraus und können von zahlreichen Providern nicht im Rahmen der üblichen Geschäftsabwicklung implementiert werden. Die in den letzten Jahren neu entwickelten hybriden Sperrtechnologien kombinieren die Vorteile beider Ver-

\section{Rechtliche Grenzen für Zugriffssperren}

Problematischer als das dargestellte Umgehungsrisiko sind die durch Zugriffssperren verursachten Eingriffe in Grundrechte. Zu berücksichtigen sind insbesondere die Berufsfreiheit und der Eigentumsschutz der Zugangsdiensteanbieter, die Meinungsfreiheit der Content-Provider sowie die Informationsfreiheit der Nutzer. Diese - in Rechtsprechung und Literatur auch bisher schon diskutierten Eingriffe - wurden in der Untersuchung des Max-Planck-Instituts detailliert analysiert.

Ein neuer verfassungsrechtlicher Schwerpunkt der Untersuchung ergab sich aus der technischen Erkenntnis, dass die Umsetzung von Sperrverfügungen in vielen Fällen durch die Analyse der angeforderten IP-Adressen und URLs auch in das Fernmeldegeheimnis eingreift. Das beruht darauf, dass dieses Grundrecht nicht nur den Inhalt, sondern auch die näheren Umstände der Kommunikation schützt. Darunter fallen nach den Ergebnissen der Studie auch die Adressen und Port-Nummern, die bei den meisten Sperrtechnologien flächendeckend ausgewertet werden fahren, indem sie zunächst eine grobe Filterung auf „verdächtige“ (IP-)Adressen vornehmen und dann nur diese vorselektierte Kommunikation im Hinblick auf URL-Adressen noch weiter analysieren. Das erfordert allerdings eine Infrastruktur zur Überwachung des Internetverkehrs, die mit beträchtlichen Eingriffen in das Fernmeldegeheimnis und Missbrauchsrisiken verbunden ist.

Zudem können (mehr oder weniger) fachkundige Nutzer sämtliche geschilderten Sperren umgehen. Insoweit spielen die jeweils vorhandene Netzinfrastruktur (mit verschiedenen Zugangsvermittlern im Inland, einer möglichen Einwahl über ausländische Access-Provider oder dem Zugriff auf Anonymisierungsdienste) sowie das jeweilige (eher demokratische oder totalitäre) Regierungssystem eine wichtige Rolle. Gleichwohl sind Sperrmaßnahmen auch in marktwirtschaftlich geprägten und freiheitlichen Demokratien nicht völlig nutzlos oder auf eine symbolische Politik reduziert. Trotz der bestehenden alternativen $\mathrm{Zu}$ gangsmöglichkeiten werden insbesondere Kinder und Jugendliche jedenfalls vor einem zufälligen Kontakt mit schädlichen Inhalten geschützt und Nutzer ohne Kenntnisse der Umgehungsmöglichkeiten vom gewünschten Zugriff auf illegale Inhalte abgehalten.

müssen, um den Zugriff auf bestimmte Angebote zu verhindern.

Die Eingriffsintensität von Sperrmaßnahmen bei der Analyse von dem Fernmeldegeheimnis unterliegenden Daten war weder der Öffentlichkeit noch dem Gesetzgeber ausreichend bewusst. Die Legislative hat deswegen in ihrer komplizierten und höchst unübersichtlichen Regelung von Sperranordnungen nicht normiert, ob und inwieweit bei der Umsetzung in das Fernmeldegeheimnis eingegriffen werden darf. Dabei hat sie nicht nur gegen die Zitiergebote von Art. 19 Abs. 1 Satz 2 Grundgesetz und $§ 88$ Abs. 3 Satz 3 Telekommunikationsgesetz verstoßen, weil sie in der Ermächtigungsgrundlage an keiner Stelle die Möglichkeit einer Einschränkung des Fernmeldegeheimnisses erwähnt. In der Normenkette des Jugendmedienschutz-Staatsvertrages, des Staatsvertrages über Rundfunk und Telemedien und des Telemediengesetzes wurde - im Gegenteil vielmehr festgelegt, dass auch bei Sperrverpflichtungen das Fernmeldegeheimnis „zu wahren“ ist. 
Die geltende Rechtslage erlaubt somit gegenwärtig keine Sperrmaßnahmen, die in das von Art. 10 Grundgesetz und $\S 88$ Telekommunikationsgesetz geschützte Fernmeldegeheimnis eingreifen. Damit scheiden derzeit alle Ansätze - einschließlich der neuen hybriden Sperrtechniken - aus, die auf der Analyse von IP-Adressen, Port-Nummern, URLs oder Inhaltsdaten beruhen. Zulässig bleiben nur die - nicht in das Fernmeldegeheimnis eingreifenden Manipulationen von Domain-Namen an den entsprechenden Servern sowie die Unterdrückung von Einträgen in der Trefferliste von Suchmaschinen.

Diese Beschränkung der zulässigen Sperrtechniken beruht nicht nur auf einer formalen
Gesetzesanwendung. Sie ist auch in der Sache berechtigt. Insbesondere die neuen hybriden Technologien, die „grobe“ IP-Vorfilter mit feinen URL-Filtern kombinieren, ermöglichen nicht nur die Sperrung von ausländischen Internetadressen, sondern könnten mittels einer zentralen Kontrollarchitektur potenziell sogar eine effektive und flächendeckende inhaltliche Überwachung der Internetkommunikation erleichtern. Der Einsatz solcher, unter Umständen sehr eingriffsintensiven Kontrolltechniken setzt voraus, dass sich der parlamentarisch legitimierte Gesetzgeber über die damit verbundenen Einschnitte in die Freiheitsrechte seiner Bürger umfänglich im Klaren ist und ihre Verhältnismäßigkeit gründlich abwägt.

\section{Weitere Fragen und Zukunftsperspektiven}

Die Untersuchung des Freiburger Max-PlanckInstituts geht über die hier dargestellte Problematik hinaus, beleuchtet zahlreiche weitere Fragestellungen des geltenden Rechts und gibt eine Gesamteinschätzung der gegenwärtigen Rechtslage. Dabei erweisen sich die bestehenden Regelungen auch in anderen Punkten als unausgereift. Dies gilt sowohl im Hinblick auf die Effektivität als auch auf den Schutz der von Sperrverfügungen tangierten Grundrechte. Zu kritisieren sind besonders die Zuständigkeiten unterschiedlicher Behörden für Sperrverfügungen, die zukünftig zu Durchsetzungsproblemen führenden Verweisungsketten und weitere Systembrüche.

Der mangelhafte Schutz der von Sperrmaßnahmen massiv tangierten Grundrechte zeigt sich nicht nur an der fehlenden Regelung zu Eingriffen in das Fernmeldegeheimnis, sondern auch an den unzureichenden Überlegungen und Normen zu Transparenz, Kontrolle und spezifischen Rechtsschutzmöglichkeiten sowie an der nicht vorhandenen Kostenregelung. Bei der Durchführung von nationalen Sperrmaßnahmen im Internet geht es um derart zentrale Fragestellungen für die Freiheitsrechte der Bürger und die Integrität des gesamten Internets, dass der Aufbau eines einigermaßen effektiven Systems auch insoweit nicht ohne intensive Überlegungen und Leitentscheidungen des Gesetzgebers erfolgen darf.
Will die Legislative eine - in ihren Erfolgsaussichten gleichwohl fragliche - wirkungsvollere, präzisere, transparente und kontrollierte nationale Abschottung gegen illegale Inhalte im Internet ermöglichen, so sind gesetzliche Neuregelungen notwendig, die sich keinesfalls nur auf die Gestattung von Eingriffen in den Fernmeldeverkehr beschränken dürfen. Dies erfordert eine Grundsatzdiskussion über technische Konzepte zur „Territorialisierung des Internets“ in freiheitlichen Gesellschaften, über die jeweiligen rechtlichen Möglichkeiten und ihre Begrenzung durch Freiheitsrechte sowie vor allem auch über alternative Schutzstrategien. Diese Grundsatzdiskussion muss vor entsprechenden Reformschritten geführt werden.

Die Ergebnisse dieser Studie zeigten bereits kurz nach ihrer Fertigstellung Wirkung: Der Präsident der Kommission für Jugendmedienschutz, die bei der Vergabe des Gutachtens noch einer Politik der nationalen Sperrverfügung zugeneigt war, erklärte auf der Pressekonferenz zur Vorstellung der Studie am 28.4.2008 in einer vielzitierten Stellungnahme, die KJM wolle aufgrund der Gutachtenergebnisse Sperrverfügungen nunmehr allenfalls als ultima ratio einsetzen.

Ulrich Sieber, Malaika Nolde 


\title{
C. Terrorismusbekämpfung jenseits der funktionalen Grenzen des Strafrechts
}

\author{
Kontrollverfügungen gegen mutmaßliche Terroristen im Vereinigten Königreich
}

Strafrechtliche

Forschungsabteilung

Forschungsschwerpunkt:

Funktionale Grenzen

des Strafrechts

Deliktsbereich:

Terrorismus

Die Terroranschläge vom 11. September 2001 haben zu neuen Strategien der Terrorbekämpfung geführt, welche der Dimension dieser Anschläge Rechnung tragen sollen. Der Einsatz von Selbstmordattentätern und die drohenden hohen Opferzahlen stellen die Politik und die Strafjustiz vor Probleme, die sich nicht mit den klassischen Methoden der - eine begangene Straftat voraussetzenden - repressiven Strafverfolgung lösen lassen. Ziel soll es deswegen sein, die Anschläge bereits im Vorfeld zu verhindern, wobei das repressive Strafrecht jedoch schon im Hinblick auf diese Aufgabenstellung an seine funktionalen Grenzen stößt. Auch müssen Freiheit und Sicherheit ausbalanciert und miteinander in Einklang gebracht werden. Die Frage nach einer Abwägung der beiden Werte stellt sich in besonderer Weise im Hinblick auf den Umgang mit Personen, die als gefährlich eingeschätzt werden, aber noch keine Straftat verübt haben.

In Deutschland gibt es in den Polizeigesetzen der Länder zwar Rechtsgrundlagen für präventive Maßnahmen, welche die Bewegungsfreiheit des Einzelnen einschränken. Dabei handelt es sich aber lediglich um die Möglichkeit kurzfristiger präventiver Freiheitsentziehungen, die darauf abzielen, unmittelbar bevorstehende schwere Straftaten zu verhindern. ${ }^{1}$ Regelungen, die eine längerfristige präventive Freiheitsbeschränkung erlauben, sieht das deutsche Recht jedoch bislang nicht vor. Mangels präventiver Möglichkeiten wird stattdessen versucht, eine größere Sicherheit durch das repressive Strafrecht zu erzielen, indem die Strafbarkeit möglichst weit in das Vorfeld verlagert wird, wenn z.B. die Ausbildung in einem terroristischen Trainingscamp oder der Besitz gefährlicher Substanzen unter Strafe gestellt wird. Das Vereinigte Königreich hat hingegen - neben zahlreichen anderen Maßnahmen - ein präventives System entwickelt, das speziell auf „terroristische Gefährder“ Anwendung findet. Durch die sogenannten Kontrollverfügungen (control orders) sollen potentiell gefährliche Personen an der Verübung von Terroranschlägen gehindert werden. ${ }^{2}$

Die Untersuchung der Kontrollverfügungen bildet einen der Schwerpunkte des Dissertationsprojekts „Eingriffe in die Bewegungsfreiheit durch die Terrorismusgesetzgebung des Vereinigten Königreichs“. In dem Projekt der strafrechtlichen Forschungsabteilung wird untersucht, wie das Vereinigte Königreich den Konflikt von Freiheit und Sicherheit in seiner Terrorismusgesetzgebung und deren Anwendung in der Praxis löst. Ein besonderer Fokus liegt auf der Frage der Vereinbarkeit verschiedener freiheitsbeschränkender Maßnahmen mit den nationalen und europäischen Grundrechtsgarantien. Zu den rechtlich interessantesten und zugleich umstrittensten Maßnahmen gehören dabei die Kontrollverfügungen. Aufbauend auf der Analyse ihrer Grundrechtskonformität wird in dem Projekt überlegt, ob das neuartige Kontrollverfügungssystem auch eine denkbare Lösung für die deutsche Strategie zur Terrorbekämpfung sein könnte. Nationale Maßnahmen zur Bekämpfung von Terrorismus werden zwar vor allem durch internationale Vorgaben beeinflusst. Die jüngere Entwicklung zeigt aber, dass auch zunehmend Lösungen anderer Rechtssysteme Beachtung finden und eine Übertragung in das eigene nationale Recht zumindest diskussionswürdig ist. So hat etwa Australien das zunächst einmalige Modell der britischen Kontrollverfügungen bereits übernommen.

\section{Das britische System der Kontrollverfügungen}

Die britischen „Kontrollverfügungen“ sind verwaltungsrechtliche Anordnungen, die präventiv eingesetzt und vom Innenminister mit der Zustimmung des Gerichts erlassen werden. Durch diese Verfügungen können Personen, die der Verstrickung in Terroraktivitäten verdächtigt werden, Auflagen erteilt werden, die sie insbesondere in ihrer Bewegungsfreiheit, aber auch ganz allgemein in ihrem alltäglichen Leben beschränken. Sie sollen als präventive Maßnahme eine weitere Einbindung 
der betreffenden Person in Terroraktivitäten einschränken oder verhindern. Hierdurch soll die Bevölkerung vor Terroranschlägen geschützt werden. Grundsätzlich sollen Kontrollverfügungen nur dann eingesetzt werden, wenn die Strafverfolgung einer Person nicht möglich ist. Insoweit sind sie als nachrangiges bzw. letztes Mittel zur Terrorbekämpfung vorgesehen. Gleichzeitig dienen sie aber dazu, eine Sicherheitslücke zu schließen, die im Hinblick auf Möglichkeiten zur Vermeidung von Terroranschlägen besteht. Dies ist insbesondere den Besonderheiten des englischen Strafprozessund Beweisrechts geschuldet. Nicht nur Geheimdienstinformationen, sondern auch Erkenntnisse, die aus geheimen Überwachungsmaßnahmen wie einer Telefonüberwachung stammen, sind bislang nicht als Beweismittel im Strafprozess zugelassen, sodass auf dieser Grundlage auch keine Strafverfolgung initiiert werden darf. Kontrollverfügungen sollen deswegen dann verhängt werden, wenn Informationen vorliegen, die einen hinreichenden Verdacht begründen, dass die betreffende Person in Terroraktivitäten verstrickt ist, diese aber vor Gericht unverwertbar sind und daher eine Strafverfolgung nicht möglich ist.

Das Verfahren zum Erlass und zur Überprüfung von Kontrollverfügungen ist als spezielles verwaltungsrechtliches Verfahren ausgestaltet, das die Einführung und Berücksichtigung von entsprechendem Material gestattet, ohne dass dies dem Betroffenen, dessen Rechtsbeistand oder der Öffentlichkeit offen gelegt wird und damit staatliche Sicherheitsinteressen verletzt werden. Dies erfolgt in sogenannten „geschlossenen Sitzungen“, an denen Anwälte teilnehmen, die zuvor eine Sicherheitsüberprüfung durchlaufen haben und durch deren Einsatz die Fairness des Verfahrens gewahrt werden soll.

Kontrollverfügungen können seit März 2005 auf der Grundlage des Prevention of Terrorism Act 2005 (PTA 2005) erlassen werden. Zuvor war zunächst im Dezember 2001 die Möglichkeit einer zeitlich unbefristeten Inhaftierung von ausländischen Terrorverdächtigen (indefinite detention) geschaffen worden, die weder angeklagt noch in ihr Heimatland abgeschoben werden konnten. ${ }^{3}$ Diese Regelung wurde jedoch im Dezember 2004 vom House of Lords, dem obersten Gericht, als mit der EMRK nicht vereinbar erklärt. Als Hauptkritikpunkt wurde die Ungleichbehandlung zwischen nicht-britischen und britischen Terrorverdächtigen genannt. Jedoch war auch die Vereinbarkeit mit Art. 5 EMRK, der das Recht auf Freiheit und Sicherheit garantiert, umstritten.
Uneinigkeit herrschte darüber, ob die Bedrohung durch den Terrorismus einen öffentlichen Notstand darstellte, der zum Abweichen von der EMRK berechtigte. Einhellig wurde die Maßnahme aber als unverhältnismäßig erachtet. ${ }^{4}$

Der PTA 2005 sieht zwei Arten von Kontrollverfügungen vor: zum einen Verfügungen, deren Auflagen mit der EMRK vereinbar sein sollen (und die als non-derogating control orders bezeichnet werden), zum anderen solche, die dem Betroffenen Auflagen machen, welche mit der EMRK unvereinbar sind (die sogenannten derogating control orders). Eine derogating control order kann nur dann erlassen werden, wenn das Vereinigte Königreich zuvor eine förmliche Erklärung über das Vorliegen eines öffentlichen Notstands abgegeben und deshalb die Abweichung von Konventionsrechten nach Art. 15 EMRK erklärt hat. Bislang wurden ausschließlich Kontrollverfügungen erlassen, bei deren Erlass der Innenminister die Auflagen für vereinbar mit der EMRK hielt. Diese Klassifizierung der Kontrollverfügungen besagt jedoch nicht, dass die Auflagen auch tatsächlich mit der EMRK im Einklang stehen. Dies ist vielmehr für jeden Einzelfall zu prüfen.

Durch eine Kontrollverfügung können dem Verfügungsadressaten, d.h. der kontrollierten Person, verschiedenste Auflagen gemacht werden. Der jeweilige Inhalt der Verfügung und die Auflagen werden individuell auf die von dem Terrorverdächtigen vermeintlich ausgehenden Risiken zugeschnitten. Der PTA 2005 enthält einen Katalog von Beschränkungen und Pflichten, die eine Kontrollverfügung beinhalten kann, jedoch ist dieser Katalog nicht abschließend. ${ }^{5}$ Typische Auflagen umfassen Beschränkungen der Bewegungsfreiheit und des Aufenthaltsorts; diese sind häufig mit der Verhängung von täglichen Ausgangssperren verbunden. Daneben können der Besitz und die Benutzung bestimmter Kommunikationsmittel wie etwa von Mobiltelefonen oder Computern untersagt sowie der persönliche Umgang reglementiert werden. Auch werden häufig Meldepflichten und das Tragen einer elektronischen Kennzeichnung, ähnlich einer elektronischen Fußfessel, auferlegt, um die Befolgung der Auflagen zu überwachen. Weiter können Auflagen gemacht werden, die den Betroffenen verpflichten, mit den Behörden zusammenzuarbeiten und ihnen jederzeit Zutritt zu seiner Wohnung zwecks Durchsuchung der Räume zu gewähren. Zuwiderhandlungen gegen eine Kontrollverfügung sind Straftaten, die mit bis zu fünf Jahren Freiheitsstrafe geahndet werden können. 


\section{Vereinbarkeit von Kontrollverfügungen mit der EMRK}

Kontrollverfügungen werfen aus menschenrechtlicher Sicht eine Reihe von Problemen auf. Zum einen stellt sich die Frage, wie weit die Auflagen eine Person in ihrer persönlichen Freiheit einschränken dürfen, ohne dass dies als willkürliche Entziehung der Freiheit und damit als eine Verletzung von Art. 5 EMRK anzusehen ist. Anhand der ersten Entscheidungen britischer Gerichte wurde deutlich, dass Kontrollverfügungen eine Gratwanderung bezüglich ihrer Vereinbarkeit mit Art. 5 EMRK bedeuten. ${ }^{6}$ In einigen Fällen wurde zwar eine konventionswidrige Freiheitsentziehung bejaht, diese aber vor allem mit der Dauer der auferlegten Ausgangssperre begründet, die in einigen Fällen bis zu 18 Stunden am Tag dauerte. Ob eine kürzere Ausgangssperre automatisch bedeutet, dass eine Kontrollverfügung das Recht auf Bewegungsfreiheit nicht verletzt, bleibt hingegen offen. Die besondere Eingriffsintensität einer Kontrollverfügung beruht auf dem Zusammenspiel verschiedenster Beschränkungen, die dem Betroffenen oftmals kein normales Leben mehr ermöglichen. Dies kann aus menschenrechtlicher Sicht unter zahlreichen Gesichtspunkten kriti- siert werden. Kontrollverfügungen erinnern in ihrem Inhalt und ihrer Wirkung an Maßnahmen, die typischerweise als Bewährungsauflagen verhängt werden. Sie werden jedoch ohne vorheriges Strafurteil durch die Exekutive auf der Grundlage eines relativ geringen Verdachtsgrades (reasonable suspicion) erlassen. Ferner ist auch zu berücksichtigen, dass Kontrollverfügungen zunächst für ein Jahr verhängt werden können, sie anschließend jedoch unbegrenzt oft jeweils um ein weiteres Jahr verlängerbar sind, sofern die Auflagen weiterhin zum Schutz der Öffentlichkeit vor Terroranschlägen erforderlich scheinen.

Neben der Frage der Vereinbarkeit mit Art. 5 EMRK wirft das Kontrollverfügungssystem auch Fragen bezüglich der Fairness des Verfahrens nach Art. 6 EMRK auf. Dadurch, dass es sich nicht um ein strafrechtliches Verfahren handelt, sondern um ein Verwaltungsverfahren zum Erlass einer präventiven Maßnahme, werden die zusätzlichen Schutzrechte umgangen, die einem Angeklagten nach Art. 6 Abs. 2 und 3 EMRK zustehen.

\section{Kontrollverfügungen - ein nachahmenswertes Modell?}

Die britischen Gesetze räumen mit den Kontrollverfügungen der Sicherheit und der Vorbeugung höchste Priorität ein. Aus menschenrechtlicher Sicht ist dies problematisch, weil durch die Kontrollverfügungen Terrorverdächtige auf der Basis eines relativ geringen Verdachtsgrads dauerhaft massiven Einschränkungen ihres alltäglichen Lebens unterworfen werden können und damit im Einzelfall ihr Recht auf persönliche Freiheit verletzt wird. Die britischen Kontrollverfügungen lösen den Konflikt zwischen Freiheit und Sicherheit nicht in zufriedenstellender Weise, da sie überaus eingriffsintensive Maßnahmen ermöglichen, deren
Vereinbarkeit mit der EMRK sehr fragwürdig ist. Daher sollten sie nicht als Vorbild für Anti-TerrorMaßnahmen anderer Staaten dienen. Unabhängig von der Problematik der Grundrechtskonformität, müsste für eine mögliche Übertragung des Kontrollverfügungssystems auf andere Rechtsordnungen außerdem berücksichtigt werden, dass die Kontrollverfügungen in ihrer derzeitigen Ausgestaltung auch den Besonderheiten des englischen Prozess- und Beweisrechts geschuldet sind.

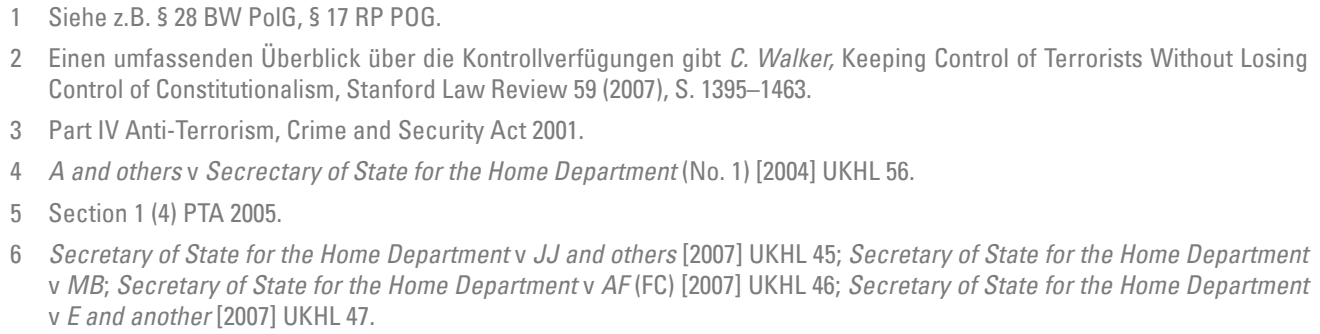

2 Einen umfassenden Überblick über die Kontrollverfügungen gibt $C$. Walker, Keeping Control of Terrorists Without Losing Control of Constitutionalism, Stanford Law Review 59 (2007), S. 1395-1463.

6 Secretary of State for the Home Department v JJ and others [2007] UKHL 45; Secretary of State for the Home Department v MB; Secretary of State for the Home Department v AF(FC) [2007] UKHL 46; Secretary of State for the Home Department $\checkmark$ E and another [2007] UKHL 47. 


\title{
D. Neue informationstechnische Überwachungsmaßnahmen
}

\author{
Die Online-Durchsuchung vor dem Bundesverfassungsgericht
}

\begin{abstract}
Das Verfahren vor dem Bundesverfassungsgericht gegen die Regelung von Online-Durchsuchungen im nordrhein-westfälischen Verfassungsschutzgesetz verdeutlicht die zentrale Herausforderung des Strafrechts durch den Wandel der globalen Informations- und Risikogesellschaft, dessen rechtliche Auswirkungen im Mittelpunkt des strafrechtlichen Forschungsprogramms stehen. Auf der einen Seite werden unter Hinweis auf zunehmende Risiken des Terrorismus und anderer Formen komplexer Kriminalität neue und erweiterte Eingriffsbefugnisse gefordert. Die Gegenposition macht demgegenüber geltend, dass gerade diese Eingriffe die vom Strafrecht geschützte Freiheit des Bürgers beseitigen. Beide Positionen finden sich in der Politik teilweise auch in kriminalpolitischen Sicherheitsdiskursen zur Legitimation der Grenzverschiebungen des Strafrechts oder der schlichten Gewinnung von Wählerstimmen.
\end{abstract}

Das - bei der Online-Durchsuchung sichtbare - hohe Überwachungspotential der modernen Informationstechnik hat diesen Konflikt erheblich verschärft. In der politisch aufgeheizten Atmosphäre vor der Entscheidung des Bundesverfassungsgerichts wurden daher auch überwiegend Extrempositionen vertreten: Ein Lager verlangte pauschal nach entsprechenden Eingriffsbefugnissen; das andere lehnte Online-Durchsuchungen vollständig und für alle Fälle - auch extremer - Kriminalität ab. Wenig Gehör fanden dagegen vor der Entscheidung des Bundesverfassungsgerichts vermittelnde Positionen, die sich um Ausgleichsmechanismen zum Schutz von Sicherheit und Freiheit bemühten.

Der Leiter der strafrechtlichen Forschungsabteilung des Freiburger Max-Planck-Instituts für ausländisches und internationales Strafrecht wirkte an der mündlichen Verhandlung des Bundesverfassungsgerichts am 10. Oktober 2007 als Sachverständiger mit und trug die Gründe für eine solche Mittelposition vor, wie sie dann auch in der Entscheidung des Bundesverfassungsgerichts vertreten wurde (BVerfG NJW 2008, S. 822 ff. vom 27.2.2008). Das vollständige Gutachten ist unter http://www.mpicc.de/ shared/data/pdf/bverfg-sieber-1-endg.pdf im Internet abrufbar. Der nachfolgende Text gibt Teile davon auszugsweise und im Originaltext in vier - durch neu eingefügte Überschriften verdeutlichten - Schritten wieder; er ist daher stärker in der juristischen Diktion geschrieben als die anderen hier abgedruckten Beiträge. Die Ausführungen betreffen zunächst den Begriff des Online-Zugriffs und seine Durchführung. Anschließend folgen Feststellungen über die wachsende Bedeutung der Internetüberwachung für die Sicherheitsbehörden einerseits und ihre Risiken für die Freiheitssphäre der Bürger andererseits. Auf dieser Grundlage werden schließlich rechtliche Begrenzungen und mögliche Ausgleichsmechanismen für die - kriminalpolitisch und praktisch notwendige - Onlinedurchsuchung entwickelt.

\section{Begriff und Durchführung des Online-Zugriffs}

Mit dem Begriff der Online-Durchsuchung wird ein - meist einmaliger - heimlicher Zugriff auf fremde Computersysteme zum Zweck der Kopie gespeicherter Daten bezeichnet. Der Begriff der Online-Überwachung erfasst dagegen auch die darüber hinausgehende - mehr oder weniger andauernde - heimliche Überwachung von laufenden Aktivitäten eines Computersystems.

Die Online-Durchsuchung eines Computersystems bietet den Ermittlungsbehörden im Unterschied zu einer klassischen Durchsuchung vor allem den Vorteil des heimlichen Vorgehens, sodass Verdächtige nicht gewarnt werden und die Behörden ohne Beweismittelverlust weiter gegen sie ermitteln können. Die Online-Durchsuchung ermöglicht darüber hinaus auch den Zugriff auf einen im Netz erfassten Rechner, dessen körperlicher Standort nicht ermittelt werden kann (und der möglicherweise auch im Ausland steht).

Demgegenüber hat die auf eine Überwachung laufender Aktivitäten gerichtete Online-Überwachung eine sehr viel größere Eingriffsintensität.
Strafrechtliche Forschungsabteilung

Forschungsschwerpunkt: Funktionale Grenzen des Strafrechts

Deliktsbereich: Terrorismus und organisierte Kriminalität 
Denn diese Form der Überwachung ermöglicht den Zugriff auf zahlreiche weitere Daten und Informationen, die im Computersystem nur flüchtig gespeichert werden. Dies betrifft insbesondere die vom Computernutzer eingegebenen Kryptoschlüssel und Passwörter (welche von einem eingeschleusten Programm heimlich mitprotokolliert werden können), die vom Computernutzer kurzzeitig zwecks Bearbeitung entschlüsselten Dateien (die ebenfalls festgehalten werden können), die versteckten und ausgelagerten Dateien (deren Abruf registriert werden kann) sowie weitere nur kurzzeitig im Arbeitsspeicher befindliche Daten, die etwa bei der Internet-Telefonie, beim Chat oder bei Videokonferenzen anfallen. Im Wege einer „Fernsteuerung“ des Zielrechners können dabei nicht nur die Tastatureingaben und Bildschirmanzeigen mitverfolgt werden. Technisch ist es darüber hinaus auch möglich, das Mikrofon oder eine integrierte Kamera (Webcam) des kontrollierten Rechners heimlich einzuschalten, die Aufzeichnungen mitzuprotokollieren und für eine akustische oder räumliche Überwachung im Umfeld des überwachten Computers zu nutzen. Auch könnte der Überwacher - insbesondere bei einem Zugriff über die Fernsteuerungs- bzw. Remote-
Funktionalität des Computers - die Kontrolle über sämtliche Aktivitäten des Computersystems übernehmen und selbständig Daten verändern. Aufgrund der grenzüberschreitenden Natur des Internet lassen sich all diese Aktivitäten gleichermaßen aus dem In- und Ausland durchführen.

Das - einmalige und insbesondere auch dauerhafte - Ausspähen von Daten ist dabei vor allem auch deswegen möglich, weil der Angreifer beim erstmaligen Eindringen nicht nur Daten kopieren und sofort auf seinen Rechner übertragen kann. Er kann auch ein spezielles Computerprogramm auf dem Zielrechner installieren. Dieses Programm kann sowohl Daten (wie die Tastatureingaben des Nutzers) in einer auf dem Zielrechner verdeckt angelegten und verschlüsselten Datei sammeln als auch in dem angegriffenen Rechner eine „Hintertür“ öffnen, weitere Programmkomponenten über das Internet nachladen und so neue Instruktionen für zukünftige Aktivitäten entgegennehmen. Der Angreifer übernimmt damit zumindest teilweise die Kontrolle über das fremde System. Die dadurch gewonnenen Daten können auf diese Weise an den Angreifer versandt oder von diesem abgerufen werden.

\section{Bedeutung der Internetüberwachung für die Sicherheitsbehörden}

Die Überwachung von informationstechnischen Systemen sowie die Beobachtung des Internet hat für die Nachrichtendienste und die Strafverfolgungsbehörden zur Erfüllung ihrer Aufgaben heute eine erhebliche Bedeutung. Denn das Internet wird nicht nur zur Begehung von computerspezifischen Delikten (z.B. Hacking, Sabotage u.Ä.) benutzt, sondern auch zur Kommunikation zwischen Straftätern in vielen anderen schwerwiegenden Deliktsarten (z.B. Wirtschaftskriminalität, organisierte Kriminalität und Terrorismus). Hinzu kommt, dass im Zuge der weltweiten Globalisierung Straftaten zunehmend grenzüberschreitend geplant und durchgeführt werden. Straftäter sind deswegen auf eine effektive internationale Kommunikation angewiesen, da sie ebenso wie Unternehmen ihre Aktivitäten - oft auch grenzüberschreitend - koordinieren müssen. Eine besondere Bedeutung hat das Internet vor allem für die Werbung, Rekrutierung, Finanzierung und Ausbildung im Bereich des Terrorismus sowie für die Planung und Vorbereitung entsprechender Straftaten.

Die Probleme der Ermittlungsbehörden bestehen in diesem globalen Szenario von Kriminalität nicht nur darin, die ausgetauschten Daten auf den Endgeräten zu finden (die z.B. in Bilddateien oder nicht sichtbaren Verzeichnissen versteckt sein können) und zu entschlüsseln. Hinzu kommt, dass der Klartext von Nachrichten häufig selbst durch einen Austausch von Begriffen verschleiert wird. Beispielsweise sollen im Vorfeld der terroristischen Attentate in den USA vom 11. September 2001 die Anzahl der Attentäter und die Zahl ihrer Anschlagsziele in einer Darstellung über die Zahl von Studenten und entsprechende Fakultäten an einer Universität verschlüsselt worden sein.

Ungeachtet der Ermittlungsprobleme sprachlich und semantisch verschlüsselter Kommunikation bleibt jedoch festzuhalten, dass der Überwachungsaufwand im Internet geringer sein kann als der Aufwand für klassische Ermittlungsmaßnahmen wie z.B. die herkömmliche Telefonüberwachung. Die bereits genannten Sperr- und Überwachungstechnologien ermöglichen es, insbesondere bei den Access- und Host Providern die Kommunikation von bestimmten Absendern zu bestimmten Adressaten einschließlich der abgerufenen Inhalte zu überwachen. Diese Über- 
wachung kann neben der gezielten Auswertung von Massendaten (z.B. Durchsuchung von großen Datensätzen nach bestimmten Texten oder Bildern, wie dies bei der Verfolgung von Kinderpornographie erfolgreich praktiziert wird) wegen der virtuellen Bedingungen des Kommunikationsmediums „Internet“ viel leichter zu flächendeckenden Überwachungen eingesetzt werden, (z.B. Erfassung aller Nutzer, die eine bestimmte
WWW-Seite aufrufen) als in der physikalischen Welt. Dies wird beispielsweise an den Möglichkeiten der hybriden Sperrtechnologie deutlich, die in der Volksrepublik China zur Sperrung illegaler Internetangebote eingesetzt wird. Diese Technologie kann bei leichter Abwandlung zu einer flächendeckenden Überwachung der Internetkommunikation genutzt werden, wenn dem keine rechtlichen Grenzen gesetzt werden.

\section{Risiken von Online-Zugriffen}

Die Problematik der Online-Durchsuchung und insbesondere der Online-Überwachung liegt [...] nicht so sehr in ihren begrenzten Einsatzmöglichkeiten, sondern in ihrer hohen Eingriffsintensität im Hinblick auf eine Vielzahl von Daten: In vielen Rechnern werden beispielsweise - teilweise über Jahre - der E-Mail-Verkehr, der klassische Schriftverkehr, die Krankenkassenabrechnung, die Steuererklärung oder die Tagebuchaufzeichnungen gespeichert. Bei Ärzten, Rechtsanwälten und Steuerprüfern finden sich zahlreiche sensible Patienten- und Mandantendaten. Diese Akkumulation von Daten wird in der modernen Informationsgesellschaft künftig weiter zunehmen. Die große Menge dieser computergespeicherten Daten kann inzwischen mit indexbasierten - auf vielen Rechnern bereits installierten (integrierten) - Suchmaschinen in effektiver Weise analysiert werden. Die Eingriffsintensität von Durchsuchungen im „virtuellen Raum“ ist daher gegenüber klassischen Durchsuchungen in der „körperlichen“ Welt sowohl mit Hinblick auf die Datenmenge und die Datenqualität als auch bezüglich der einsetzbaren automatisierten Suchtechniken sehr viel größer. Diese Steigerung von Ermittlungschancen und Risiken besteht allerdings auch schon bei der klassischen offenen Durchsuchung von Datenträgern. Ein erheblicher Quantensprung im Hinblick auf die Datenmenge und die Datenqualität ergibt sich bei der OnlineÜberwachung allerdings aus dem bereits genannten andauernden Zugriff auf den Arbeitsspeicher sowie den Möglichkeiten einer längerfristigen Verlaufsüberwachung.

Sowohl technisch als auch rechtlich ist dabei eine Begrenzung der Maßnahmen auf einzelne Datenkategorien möglich, z.B. auf E-Mail-Daten, auf Daten der Internet-Telefonie, auf Bilder oder Texte. Da Dateibezeichnungen und Dateiattribute den Dateninhalt nicht eindeutig und vor allem nicht verlässlich beschreiben, sind entspechende
Differenzierungen bei der praktischen Arbeit jedoch nicht immer sicher durchzuhalten. Dies gilt vor allem dann, wenn die Täter ihre Daten tarnen, indem sie z.B. kritische Inhalte in Bildern, Musikdateien, Tagebuchaufzeichnungen oder Liebesbriefen verstecken.

Grundsätzlich können die Sicherheitsbehörden daher den Zugriff auf bestimmte Datenarten beschränken. Für die gezielte Suche nach bestimmten Informationen lassen sich auch spezielle Suchalgorithmen oder die in vielen Rechnern bereits schon vorhandenen Suchindices nutzen. Wenn mit groben Suchrastern oder mit technischen Maßnahmen versucht wird, Zugriffe auf bestimmte Inhalte (z.B. im Kernbereich der privaten Lebensführung) zu vermeiden, so ist dies jedoch aus mehreren Gründen nur begrenzt durchzusetzen, wenn das Suchverfahren effektiv bleiben soll: Zum einen können Vorgaben, die den Zugriff auf den Kernbereich von Grundrechten vermeiden sollen, von den Tätern ausgenutzt werden (indem z.B. Dateien zunächst mit einem tagebuchartigen Vorspann versehen werden). Zum anderen ist schon bei einem unbeschränkt nutzbaren Datenbestand eine effektive semantische Auswertung großer Datenmengen mit statischen und heuristischen Untersuchungsverfahren äußerst komplex und nicht besonders treffsicher. Dies ist umso schwieriger, wenn diese Verfahren in einer verdeckten Aktion auf einem fremden Rechner im Wege der Fernsteuerung durchgeführt werden müssen, weil eine Übertragung des Gesamtdatenbestandes auf das eigene Rechnersystem an der notwendigen Kapazität für den ausgeleiteten Datenstrom scheitert. Eine effektive Datenauswertung dürfte daher einen zumindest begrenzten Einsatz von Menschen statt Maschinen erfordern (z.B. für ein „Anlesen“ von Dateien), der allerdings auf einen speziell ausgewählten Personenkreises beschränkt werden kann. 
Der vorliegende Text gibt zentrale Teile des Gutachtens im Original wieder. Vgl. den vollständigen Beitrag unter http://www.mpicc. de/shared/data/pdf/bverfg-sieber1-endg.pdf sowie die Entscheidung, BVerfG NJW 2008, 822-837.

\section{Rechtliche Begrenzungen und Ausgleichsmechanismen}

Die vorangegangenen Ausführungen zeigen, dass der rechtsstaatlichen Begrenzung von OnlineZugriffen eine besondere Bedeutung zukommt. Dabei ist nicht nur im Hinblick auf OnlineDurchsuchungen und Online-Überwachungen zu differenzieren. Bei der Entwicklung von rechtlichen Begrenzungen ist auch zwischen OnlineZugriffen im Strafverfahren, in der polizeilichen Gefahrenabwehr und in der nachrichtendienstlichen Arbeit zu unterscheiden, auch wenn diese Kategorien bei neuen komplexen Kriminalitätsformen (insbesondere organisierter Kriminalität und Terrorismus) verschwimmen und in Frage gestellt werden können.

Für die verschiedenen Eingriffsnormen sind insbesondere folgende Regelungen in Betracht zu ziehen:

- Die Differenzierung nach der Zugriffsfunktionalität der Eingriffsnorm mit Blick auf Online-Durchsuchungen einerseits und Online-Überwachungen andererseits (sowie ggf. den - beide Kategorien betreffenden - Spezialfall der Quellen-TKÜ).

- Interne Begründungs- und Berichtspflichten der für die Online-Zugriffe verantwortlichen Personen. - Die tatbestandliche Begrenzung des Anwendungsbereichs der Eingriffsnorm auf einen Tatverdacht und/oder eine Gefahr von besonderer Schwere und/oder Erheblichkeit (wobei das Verschwimmen der für das Strafrecht kategorialen Unterscheidung von Repression und Prävention im Bereich komplexer Kriminalität auch eine Kombination der entsprechenden polizeilichen und strafrechtlichen Kriterien oder aber eine Grenzziehung für strafrechtliche und polizeirechtliche Maßnahmen insgesamt nahelegt).

\section{Fazit}

Die Frage nach einem Einsatz von Online-Zugriffen zu präventiven und repressiven Zwecken zeigt damit die typischen Probleme der Kontrolle und Verfolgung komplexer Kriminalität in der globalen Informations- und Risikogesellschaft: Die neuen Formen der Kriminalität (insbesondere im Bereich des Terrorismus) führen zu neuen Risiken und erheblichen Ermittlungsproblemen für den Staat. Neue informationstechnische Ermittlungsmaßnahmen können in begrenztem Maße eingesetzt werden. Sie beschränken jedoch potentiell in
- Ein bestimmter Verdachtsgrad und/oder ein bestimmter Gefahrengrad sowie das Erfordernis eines entsprechenden Nachweises durch konkrete Tatsachen.

- Ein qualifizierter Richtervorbehalt, z.B. in der Form einer aus drei Richtern bestehenden Kammer (vgl. auch Art. 13 GG).

- Die Restriktion erweiterter Ermittlungsbefugnisse bei Gefahr im Verzug z.B. unter Aufrechterhaltung eines (vereinfachten) Richtervorbehalts (soweit hinsichtlich der in aller Regel erforderlichen Voraufklärungen eine besondere Dringlichkeit überhaupt gegeben ist).

- Die Beschränkung der Zugriffe insbesondere in der richterlichen Anordnung auf bestimmte Datenarten (z.B. E-Mail, Kryptoschlüssel).

- Die Normierung von Benachrichtigungspflichten sowie - für den Fall der Nichtbenachrichtung aus ermittlungstaktischen Gründen - die Prüfung der Maßnahme durch einen Ombudsmann (entsprechend Vorbildern in ausländischen Rechtsordnungen).

- Die besondere Regelung von Beweiserhebungsund Beweisverwertungsverboten, die sich z.B. auf die Daten von Berufsgeheimnisträgern und auf Daten im Kernbereich von Grundrechten beziehen.

- Besondere personelle Anforderungen für die Durchsicht von Daten.

- Die zeitliche Begrenzung von Online-Überwachungen (z.B. ähnlich wie bei der Wohnraumüberwachung).

- Vorschriften über die Protokollierung und Dokumentation der Durchführung von Maßnahmen und die Hinzuziehung von Zeugen.

- Kontrollen von unabhängigen Dritten (z.B. Parlamentsabgeordnete, Datenschützer oder spezielle Ombudspersonen)

erhöhtem Maße die bürgerlichen Freiheitsrechte. Das Verfassungsrecht und die Kriminalpolitik stehen damit vor der rechtlichen Herausforderung, diese neuen informationstechnischen Ermittlungsmaßnahmen durch den präzisen Einsatz herkömmlicher und neuer rechtsstaatlicher Ausgleichsmechanismen auf die richtigen Verdachtsund Gefahrenkonstellationen zu begrenzen.

Ulrich Sieber 


\section{E. Aktuelles Zeitgeschehen}

Transnationale Steuerermittlungen in Liechtenstein

Das Forschungsprogramm des Freiburger Max-Planck-Instituts für ausländisches und internationales Strafrecht konzentriert sich auf die rechtlichen Folgen des sozialen Wandels und - in der strafrechtlichen Abteilung - insbesondere auf die Forschungsschwerpunkte der territorialen und funktionalen Grenzen des Strafrechts. Das Institut arbeitet damit zu aktuellen Entwicklungen und zentralen Reformfragen und bezieht in wichtigen Fragen auch kurzfristig Stellung zum Zeitgeschehen. Dies zeigt sich nicht nur in seinen Stellungnahmen für die Verfahren des Bundesverfassungsgerichts zur OnlineDurchsuchung und zur Inzeststrafstrafbarkeit sowie der damit verbundenen Öffentlichkeitsarbeit. Eine aktuelle Stellungnahme in einem gleichermaßen bedeutenden Fall betraf auch die in den Jahren 2006 und 2007 betriebenen deutschen Ermittlungen gegen Liechtensteiner Banken.

Der Sachverhalt und die Ermittlungsmethoden des Liechtensteiner Falls verdeutlichen anschaulich die Aktualität, die Relevanz und die gegenseitigen Beziehungen der beiden oben genannten Forschungsschwerpunkte, deren Grundlagenfragen hinter den einschlägigen technischen und gesetzlichen Problemen stehen: Die Globalisierung unter Beibehaltung von unterschiedlichem nationalem Recht begünstigt die transnationale Steuerflucht von Deutschland nach Liechtenstein. Dies führt das nationale deutsche Strafrecht wegen der fehlenden Rechtshilferegelungen an seine territorialen Grenzen. Die Praxis entwickelt mit dem Kauf von strafrechtlich geschützten Bankdaten durch Steuerbehörden und BND neue - unkonventionelle - Maßnahmen eines transnational wirksamen Straf- und Polizeirechts. Diese Probleme und die neuen Ermittlungsmethoden stellen gleichzeitig - wie im Forschungsprogramm analysiert - die funktionalen Grenzen des Strafrechts in Frage, vor allem mit der „Vergeheimdienstlichung“ des Strafverfahrens, der Zusammenarbeit und Datenweitergabe zwischen Verwaltungsbehörden, Strafverfolgungsbehörden und Geheimdiensten, der „Privatisierung“ des Strafverfahrens durch eine nichtstaatliche extraterritoriale Informationsbeschaffung sowie der Auflösung schützender Formen des Strafprozesses durch die Inanspruchnahme von neuen Eingriffsmaßnahmen, die gesetzlich nicht vorgesehen sind und allenfalls über Notstandsbefugnisse gerechtfertigt werden könnten (vgl. zu dieser Entwicklung das Forschungsprogramm in ZStW 119 [2007], S. 1 , 27-37).

Rechtspolitisch lässt sich darüber streiten, ob solche Ermittlungsmaßnahmen gesetzlich erlaubt werden sollten. Vom geltenden Recht ist ein solches Vorgehen jedoch nicht gedeckt. Aus dem Institut kam deswegen eine kritische Stellungnahme zu dieser aktuellen Problematik schon kurz nach Bekanntwerden des Falls nicht nur in dem ersten Aufsatz zu diesem Fall, sondern auch in einem längeren Namensbeitrag in der FAZ. Der im Folgenden (mit eingefügten Zwischenüberschriften) abgedruckte Originalbeitrag aus der FAZ veranschaulicht die Bedeutung der kleineren Arbeiten des Instituts für sein Forschungsprogramm und seine Öffentlichkeitsarbeit.

\section{Sachverhalt und Problemstellung: Transnationale Steuerflucht und transnationale Beweiserhebung}

Der Kauf von Kontendaten einer Liechtensteiner Bank durch deutsche Steuerbehörden in Zusammenarbeit mit dem Bundesnachrichtendienst (BND) hat zu einer kontroversen Diskussion über die Bewertung dieses Verhaltens geführt. Die Liechtensteiner Strafverfolgungsbehörden fahnden öffentlich nach dem Verkäufer der Daten und prüfen auch, ob sich deutsche Beteiligte strafbar gemacht haben. In Deutschland wurden von dritter Seite Strafanzeigen gegen Vertreter der Bundesregierung und des Bundesnachrichtendiensts gestellt.

Nach dem derzeit bekannten Sachverhalt geht es vor allem um Daten, die ein Mitarbeiter der LGT-
Strafrechtliche

Forschungsabteilung

Forschungsschwerpunkt:

Territoriale und funktionale Grenzen des Strafrechts

Deliktsbereich: Wirtschaftskriminalität 
Bank heimlich kopiert und nach seinem Ausscheiden aus der Bank im Jahr 2006 dem BND angeboten hatte. Nach ersten „Probelieferungen“ an den deutschen Auslandsgeheimdienst und auch an die Steuerbehörden soll der Hinweisgeber dann 2007 gegen ein Entgelt von mehr als 4 Millionen Euro mehrere Datenträger mit Angaben über 4527 Begünstigte von Liechtensteiner Stiftungen und zu Anlagen in Höhe von etwa 3,5 Milliarden Euro an die Steuerbehörden verkauft haben.

Eine rechtliche Bewertung dieser Abläufe ist nicht nur wegen des ungeklärten Sachverhalts prob- lematisch. Es stellen sich auch zahlreiche neue Rechtsfragen. Gegenwärtig kann daher lediglich erörtert werden, wie die in der Öffentlichkeit „gefühlten Grenzverletzungen“ nach geltendem Recht zu bewerten sind. In Frage stehen vor allem die Rechtmäßigkeit der Weitergabe von geheimen Kontendaten durch den Bankmitarbeiter, des entgeltlichen Erwerbs von Bankgeheimnissen durch die Steuerbehörden sowie der Dienstleistungen des BND für die Steuerbehörden. Im Fall von Rechtsverstößen stellt sich auch die Frage nach eventuellen Auswirkungen auf die Verwertbarkeit der erlangten Informationen.

\section{Die Beweiserlangung des Bankangestellten: Privatisierung und „Outsourcing" der strafbaren Beweiserhebung}

Wenn ein ehemaliger Mitarbeiter der LGT-Bank deutschen Steuerbehörden Datenträger mit den von ihm entwendeten Kontendaten zu einem Preis von mehr als vier Millionen Euro verkauft, so verstößt dies nicht nur gegen Liechtensteiner, sondern auch gegen deutsche Strafbestimmungen. Soweit es um unkörperliche Informationen auf einem eigenen Datenträger des Mitarbeiters geht, kommt in Deutschland allerdings nicht wie vielfach angenommen - Hehlerei in Betracht. Gegenstand der Hehlerei können nur körperliche Sachen sein. Die bloße Kopie der immateriellen Daten erfüllt jedoch vor allem den Tatbestand einer strafbaren Verwertung von Geschäftsgeheimnissen nach Paragraph 17 Absatz 2 Nummer 2 des Gesetzes gegen den unlauteren Wettbewerb (UWG).

Die unbefugte Geheimnisverwertung ist mit einer Freiheitsstrafe bis zu drei Jahren bewehrt und erfasst alle Arten von Geschäfts- und Betriebsgeheimnissen. Dazu gehören nicht nur Hochtechnologie-Erfindungen, sondern auch Geschäftgeheimnisse, wie sie in dem López-Fall im Jahr 1993 möglicherweise mit dem Chefeinkäufer von General Motors zu VW wechselten. Unter die Vorschrift fallen auch Bankgeheimnisse und insbesondere Kontendaten, deren Schutzbedürfnis das Bundesverfassungsgericht in seiner Entscheidung über die Online-Durchsuchung soeben besonders gewürdigt hat. Das UWG bezieht dabei auch Geheimnisse ausländischer Banken ein, da das deutsche Strafrecht inländische und ausländische Individualinteressen gleichermaßen schützt. Ist ein Hinweisgeber nicht Deutscher, so unterfällt seine Tat insbesondere dann dem deut- schen Strafrecht, wenn strafbare Handlungen in Deutschland erfolgten.

Die strafbare Geheimnisoffenbarung des Mitarbeiters lässt sich in einem solchen Fall nicht mit dem allgemeinen Rechtfertigungsgrund des Notstands (Paragraph 34 Strafgesetzbuch) ausschließen, der die Verletzung einer Strafbestimmung zur Rettung eines höherwertigen Gutes erlaubt. Eine solche Rechtfertigung einer privaten Unterstützung der Strafverfolgung wird in der Strafrechtswissenschaft zu Recht überwiegend abgelehnt, da die staatliche Strafverfolgung nach dem abschließend normierten Strafprozessrecht zu erfolgen hat. Die Berufung auf Notstandsregeln wäre darüber hinaus im Hinblick auf die erforderliche Interessenabwägung und die Angemessenheit der Nothilfehandlung fragwürdig, vor allem wenn auch Kontendaten von steuerehrlichen Personen und Stiftungen weitergegeben wurden.

Aufgrund dieser Gesichtspunkte lässt sich das Verhalten des Hinweisgebers auch nicht mit einem „Recht zur Strafanzeige“ legitimieren. Die Rechtsprechung der Arbeitsgerichtsbarkeit gibt dem „whistleblower“ eine solche Befugnis nur in bestimmten Grenzen. Ein Recht zur Strafanzeige kann einen Verstoß gegen Geheimschutzstrafbestimmungen vor allem dann nicht rechtfertigen, wenn hierfür ein hoher Geldbetrag als Gegenleistung gefordert wird. Diese Bewertung lässt sich auch nicht dadurch umgehen, dass bestimmte Informationen als „illegale Geheimnisse“ pauschal dem Schutz der Strafvorschrift entzogen werden. Für den vorliegenden Sachverhalt ist deswegen von einer Strafbarkeit des Hinweisgebers auszugehen. 


\section{Ermächtigungsgrundlage der Steuer(straf)behörden:}

Der Verlust schützender Formen

Fördern deutsche Steuerbehörden eine solche strafbare Geheimnisverwertung, indem sie zur Probelieferung von Bankdaten auffordern und Geld versprechen, so verwirklichen sie den Tatbestand einer Beihilfe zu diesem Delikt. Ob sich die Behördenvertreter deswegen strafbar machen, hängt allerdings noch entscheidend davon ab, ob ihre Handlungen durch Amtsbefugnisse gerechtfertigt sind. Soweit es nur um die Aufforderung zur Datenübergabe geht, verfügen die Ermittlungsbehörden aufgrund der strafprozessualen und steuerrechtlichen Zeugnis- und Herausgabepflichten über die dazu notwendigen Befugnisse: In Deutschland muss ein Bankmitarbeiter den zuständigen Ermittlungsbeamten bei einer entsprechenden Aufforderung und beim Verdacht einer Straftat seine Kenntnisse preisgeben. Auch seine Datenträger können beschlagnahmt und verwertet werden, wie dies bereits in verschiedenen Fällen geschehen ist, in denen Bankmitarbeiter ihren Arbeitgeber oder dessen Kunden mit belastenden Unterlagen erpressen wollten. Wenn im vorliegenden Fall die Steuerfahndung die Daten lediglich entgegengenommen hätte, so läge daher der Gedanke an eine strafbare Beihilfe fern.

In dem Liechtensteiner LGT-Fall erfolgte beim Ankauf der Daten jedoch ein „Mehr“ an Handlungen, das unter dem Gesichtspunkt der Beihilfe zu Paragraph 17 UWG relevant ist: Dieses „Mehr“ ist die in der Strafprozessordnung nicht vorgesehene Zahlung eines hohen Geldbetrages, der für eine strafbare Geheimnisoffenbarung gezahlt wird und diese fördert. Da ein solches Vorgehen der strafrechtlichen Ermittlungsbehörden im geltenden Recht keine Begründung findet, entfällt der Beihilfevorwurf insoweit nicht. Die Steuerbeamten können die finanzielle Förderung der Geheimnisverwertung auch nicht mit dem bereits genannten Rechtfertigungsgrund des Notstands nach Paragraph 34 StGB begründen, weil die staatlichen Befugnisse zur Aufklärung von Straftaten in der Strafprozessordnung und in anderen Gesetzen abschließend geregelt sind und nicht einfach durch eine Generalklausel aus dem materiellen Strafrecht erweitert werden dürfen.

Die fehlende Rechtfertigung bedeutet allerdings noch nicht, dass die Beamten zu bestrafen sind. Die Neuartigkeit der auftretenden Probleme, die in vielen Punkten unklare Rechtslage und die Vertretbarkeit auch anderer Lösungen kann im vorliegenden Fall die Schuld der handelnden Personen aufgrund eines sogenannten Verbotsirrtums nach Paragraph 17 StGB entfallen lassen, soweit die falsche rechtliche Einschätzung unvermeidbar war.

Dass die Beamten damit nicht gerechtfertigt, sondern gegebenenfalls nur entschuldigt sind, ist kein akademisches Glasperlenspiel, sondern hat wichtige Konsequenzen: Die Beurteilung des Verhaltens als rechtswidrig ist nicht nur für die unten erörterte Verwertbarkeit der erlangten Beweise relevant. Sie ist auch entscheidend für die Frage, ob eine Geldzahlung der Behörden zum Zweck der Begehung einer strafbaren Geheimnisoffenbarung nur in dem ersten Sonderfall entschuldigt wird oder zukünftig als Ermittlungsmaßnahme der Strafverfolgungsbehörden oder der Steuerbehörden erlaubt ist. Die Qualifizierung des Verhaltens als rechtswidrig entscheidet vor allem auch darüber, ob das Recht fundamentale Rechtsprinzipien aufgibt und damit auf eine Bahn gerät, auf der ein Halten schwierig ist: Es geht dabei insbesondere um den allgemeinen Grundsatz, dass staatliche Ermittler außerhalb der prozessualen Befugnisse keine Straftaten gegen individuelle Rechtsgüter begehen dürfen.

Würden einzelne Straftaten aufgrund einer notstandsähnlichen Generalklausel erlaubt, so kämen rasch andere Fälle hinzu, in denen mit einer schlichten Interessenabwägung begründet werden könnte, warum Straftaten im Interesse der Strafverfolgung und der Kriminalprävention begangen werden sollten. Dies gilt etwa für die Frage, ob den Ermittlungsbehörden ein Ankauf von Bankdaten nur bei speziellen ausländischen, bei allen ausländischen oder auch bei inländischen Banken möglich sein soll. Ähnliche Probleme könnten sich auch im Hinblick auf eine Auslobung von Geld an das ärztliche Hilfspersonal für die Offenbarung von sozialschädlichem Abrechnungsbetrug stellen. Auch die Mitarbeiter von Anwaltskanzleien könnten auf den Gedanken kommen, ihre Mandatsgeheimnisse in Fällen schwerer Kriminalität unter Berufung auf einen „Ermittlungsnotstand“ dem Staat zu verkaufen. Diskutiert würde auch wieder die Frage nach der Gestattung von „milieubedingten Straftaten“, mit denen V-Leuten und verdeckten Ermittlern der Zugang zu Straftätergruppen erleichtert werden sollte.

Die Beispiele zeigen, dass der Ankauf von Geschäftsgeheimnissen unter Verletzung von Strafvorschriften ein Eingriff in fremde Rechte ist, der 
ebenso wie andere strafprozessuale Ermittlungsmaßnahmen eine gesetzliche Grundlage erfordert und im Fall seiner Anordnung durch rechtsstaatliche Sicherungen (wie einen Richtervorbehalt) kontrolliert werden muss. Wenn der Rechtsstaat den Ermittlungsbehörden ohne derartige Ermächtigungen die Verwirklichung von Straftatbeständen grundsätzlich verbietet, vermeidet er daher nicht nur, dass rechtsstaatliche Prinzipien und schützende Formen aufgelöst werden. Er begründet damit auch seine ethische Überlegenheit. Bei der vorliegenden Frage nach einer staatlichen Bezahlung eines strafbaren Geheimnisverrats geht es daher nicht nur um die wirtschaftliche Abwägung von Kosten und Nutzen, sondern um fundamentale Prinzipien des Rechtsstaats.

\section{Die „Amtshilfe“ des BND für die Steuer(straf)behörden:}

\section{„Vergeheimdienstlichung ${ }^{66}$ als Teil der neuen Sicherheitsarchitektur}

Vor ähnlich grundsätzliche Fragen stellt auch die Beteiligung des Bundesnachrichtendiensts an der Aufklärungsarbeit der Steuerbehörden. Nach dem, was bisher bekannt geworden ist, müssen drei verschiedene Handlungen des BND geprüft werden: die Erlangung von „Probedaten“, die Weiterleitung der ermittelten Informationen an die Steuerbehörden sowie die Unterstützung der Steuerbehörden beim Ankauf der Datenträger.

Die Erlangung von Probedaten durch den BND wirft zunächst die Frage nach dessen Zuständigkeit und Befugnissen auf. Solange der BND zum Zweck der außen- und sicherheitspolitischen Aufklärung etwa von internationaler Geldwäsche oder internationalem Terrorismus ermittelt, ist er nach dem BND-Gesetz zuständig. Die einschlägigen Befugnisse zum Einsatz von „nachrichtendienstlichen Mitteln“ im Sinn des BND-Gesetzes gehen auch über die Befugnisse der Steuerbehörden hinaus und erlauben in bestimmtem Umfang, dass ein Entgelt für die Verletzung von Geheimschutzbestimmungen in Aussicht gestellt oder gezahlt wird. Ohne solche Befugnisse wäre eine effektive nachrichtendienstliche Aufklärung vor allem im Ausland kaum möglich. Der BND handelt daher bei entsprechenden finanziellen Angeboten rechtmäßig, solange er gezielt außen- oder sicherheitspolitische Aufklärung betreibt und nicht von vornherein Steuerdelikte ermittelt.

Dies bedeutet aber nicht, dass der BND den Steuerbehörden alle erlangten Informationen und steuerlichen „Beifänge“ seiner außen- und sicherheitspolitischen Ermittlungen weitergeben darf. Zwar könnte der Wortlaut von Paragraph 9 Absatz 1 BND-Gesetz und Paragraph 116 Abgabenordnung $(\mathrm{AO})$ eine solche Weitergabe erlauben. Wegen der besonderen Aufklärungsbefugnisse und der geringeren Kontrolle des BND im Vergleich zu den strafrechtlichen Ermittlungsbehörden unterliegt die Weitergabe jedoch verfassungsrecht- lichen Begrenzungen. Das Bundesverfassungsgericht begründet diese Beschränkungen zutreffend damit, dass „grundrechtsgebotene Beschränkungen des Einsatzes bestimmter Erhebungsmethoden ... nicht dadurch umgangen werden (dürfen), dass Daten, die mit einer solchen Methode rechtmäßigerweise zu bestimmten Verwendungszwecken erhoben worden sind, in gleicher Weise auch für Zwecke zugänglich gemacht werden, die einen derartigen Methodeneinsatz nicht rechtfertigen würden“.

Soweit die Befugnisse des BND es rechtfertigen, dass Bankinformationen oder Probedaten durch staatliche Vergütungen für strafbaren Geheimnisverrat erlangt wurden, muss die Verwendung der erlangten Ergebnisse daher auf Fälle der schweren Kriminalität beschränkt werden, wie dies vom G 10-Gesetz bei der Telekommunikationsüberwachung geregelt ist. Andere Daten dürfen entweder gar nicht weitergegeben werden oder sie können wenn eine Trennung der unterschiedlichen Daten zunächst nicht möglich ist - nach dem Lösungsmodell des G 10-Gesetzes später nicht verwertet werden.

Für den BND stellt sich auch noch die Frage, ob er die Steuerbehörden beim Ankauf der Datenträger im Wege der Amtshilfe unterstützen konnte und ob dies die Handlungen der Steuerbehörden rechtfertigt. Hier gilt, dass Amtshilfe eine gesetzlich beschränkte Handlungsbefugnis der ersuchenden Finanzbehörde nicht erweitern darf. Freiheit, die mit der Gewaltenteilung in einem gegliederten Staat garantiert ist, darf nicht einfach durch eine beliebige Zusammenarbeit unterschiedlicher Behörden eingeschränkt werden. Amtshilfe des BND kann daher nicht dazu führen, dass die Steuerbehörden eine Befugnis zum entgeltlichen Erwerb der durch Strafvorschriften geschützten Daten erhalten, die sie ohne Amtshilfe nicht haben. 


\section{Die Früchte des verbotenen Baumes}

Nach der Bewertung der Ermittlungsmaßnahmen stellt sich damit abschließend noch die Frage nach den Konsequenzen für die Verwertbarkeit der erlangten Beweise: Ob eine strafbare oder rechtswidrige Beweisgewinnung auch zu dem Verbot führt, die erlangten Beweise im Strafverfahren zu verwerten, hängt vor allem davon ab, wer die Verstöße begeht und wem sie zuzurechnen sind. Nur in besonderen Fällen, die hier nicht vorliegen, folgt aus einer strafbaren Beweisverschaffung durch Privatpersonen, dass die erlangten Beweise nicht verwertet werden können. Anders verhält es sich, wenn wie hier das Verhalten den staatlichen Behörden zurechenbar ist. Allerdings führt dabei nicht jeder Verfahrensverstoß zu einem Beweisverwertungsverbot. Ein Beweisverwertungsverbot besteht nur aus übergeordneten wichtigen Gründen, die im Einzelfall durch Abwägung der betroffenen Interessen zu klären sind. Zu den anerkannten Fallkonstellationen zählen insbesondere bewusst rechtswidrig verschaffte Informationen und objektiv willkürliches Verhalten. Für den entgeltlichen Erwerb der Daten ist nach dem bisher bekannt gewordenen Sachverhalt fraglich, ob sich die Ermittlungsbehörden - vor allem auch subjektiv - so verhalten haben.

Für die erwähnte Weitergabe der Erstinformationen und der Probedaten vom BND an die
Steuerbehörden lassen sich dagegen andere Gesichtspunkte heranziehen: Soweit der BND die Informationen dank seiner speziellen Befugnisse und Möglichkeiten erlangte, spricht viel für eine Beschränkung der Beweisverwertung auf Fälle der schweren Kriminalität entsprechend den Wertungen des G 10-Gesetzes.

Im Fall eines Beweisverwertungsverbots könnten allerdings die erlangten mittelbaren Beweise genutzt werden, etwa die Unterlagen, die aufgrund der Kontendaten bei den Wohnungsdurchsuchungen aufgefunden werden. Eine generelle Fernwirkung des Beweisverbots, wie sie das amerikanische Strafprozessrecht mit der „fruit of the poisonous tree doctrine“ kennt, ist in Deutschland nicht anerkannt. Die Rechtsprechung neigt vielmehr je nach „Sachlage und Art des Verstoßes“ zu einer Abwägung. Die Telekommunikationsüberwachung nach dem G 10Gesetz war einer der seltenen Fälle, in denen auch wegen ihrer hohen Eingriffsintensität - eine entsprechende Fernwirkung angenommen wurde. Jedoch sind die bisherigen Ergebnisse der Rechtsprechung zu den strafrechtlichen Beweisverwertungsverboten und insbesondere zu deren Fernwirkung noch zu unbestimmt, als dass verlässliche Vorhersagen möglich wären.

\section{Konsequenzen: Die Rolle des Strafrechts und alternative Formen der Kriminalprävention}

Die Beurteilung des Liechtensteiner Falls ist damit nicht nur wegen des noch ungeklärten Sachverhalts offen, sondern vor allem auch wegen seiner Rechtsfragen. Die Aufklärung der tatsächlichen Fragen obliegt zunächst dem Parlamentarischen Kontrollgremium und dann vor allem den Gerichten. Die rechtlichen Probleme werden zu langen Diskussionen führen und können in letzter Instanz wahrscheinlich erst durch das Bundesverfassungsgericht geklärt werden. Auch unter rechtspolitischen Gesichtspunkten dürften die angesprochenen Grenzen des Strafrechts hinterfragt werden.

Bei der zukünftigen Aufarbeitung der Liechtensteiner Vorgänge muss jedoch vor allem auch der gesellschaftliche Umgang mit den offenkundig gewordenen Problemen der Steuerhinterziehung und ihrer Kontrolle diskutiert werden. Strafrecht ist zur Verhinderung von Kriminalität - ebenso wie zur Sicherung der bürgerlichen Freiheitsrechte gegen eine übermäßige Strafverfolgung - notwendig. Die Liechtensteiner Ermittlungen haben zwar unabhängig von ihrer möglichen Rechtswidrigkeit die Wirksamkeit des Steuerstrafrechts faktisch erhöht, indem sie das bestehende Aufdeckungsrisiko sichtbar gemacht haben. Selbst bei einer gesetzlichen Legitimierung der gewählten Ermittlungsmethode und der Schaffung von noch weiter reichenden staatlichen Ermittlungsbefugnissen lässt sich massenhaft begangene Steuerdelinquenz jedoch nicht allein durch Strafrecht verhindern, sondern nur auf der Grundlage von tiefgreifenden ethischen, gesellschaftlichen, wirtschaftlichen und steuerrechtlichen Veränderungen.

Ulrich Sieber
Der vorstehende Beitrag wurde in der FAZ Nr. 75 vom 31.3.2008, S. 8 als Namensbeitrag abgedruckt. Weiterführende Literatur: Ulrich Sieber, Ermittlungen in Sachen Liechtenstein, NJW 2008, 881-944. 


\title{
F. Internationale Kriminalpolitik
}

\author{
Auf dem Weg zur Abschaffung der Todesstrafe? - Strafrechtspolitik in China
}

Kriminologische

Forschungsabteilung

Forschungsschwerpunkt: Kriminalpolitik und rechtsstaatliche Entwicklung in außereuropäischen und Übergangsgesellschaften

Im Rahmen des Forschungsschwerpunkts „Kriminalpolitik und rechtsstaatliche Entwicklung in Übergangsgesellschaften“ beteiligt sich das Max-Planck-Institut für ausländisches und internationales Strafrecht an einem internationalen Projekt unter der Leitung des Great Britain China Centre (London), das eine Stärkung der Tendenzen zu einer verminderten Anwendung und langfristigen Abschaffung der Todesstrafe in China zum Ziel hat. Das Projekt „Moving the Debate Forward - China's Use of the Death Penalty“ wird im Rahmen des Programms „European Initiative for Democracy and Human Rights“ (EIDHR) von der Europäischen Union finanziell unterstützt. Als ein Element ihrer Menschenrechtspolitik unterstützt die Europäische Union weltweit Projekte zur Zurückdrängung und Abschaffung der Todesstrafe. An dem laufenden Projekt sind als chinesische Partner die Beijing Normal University und die Wuhan University sowie als weitere europäische Partner die University of Oxford (Centre for Criminology), die National University of Ireland Galway (Irish Centre for Human Rights) und das Death Penalty Project (London) beteiligt. Das Ziel des Projektes ist es, Zusammenhänge zwischen öffentlicher Meinung, Rechtspolitik, Gesetzgebung und Justizpraxis zu untersuchen und Einstellungsänderungen bei Akteuren der Politik und der Justizpraxis sowie in der Öffentlichkeit zu initiieren. Ziel ist es auch, die chinesische Strafrechtsreform - insbesondere im Hinblick auf eine Verminderung der Anzahl der Tatbestände, für die die Todesstrafe verhängt werden kann - vor dem Hintergrund der Ratifizierung des Internationalen Paktes über Bürgerliche und Politische Rechte anzuleiten.

Teil des Projektes ist eine wissenschaftliche Studie zur öffentlichen Meinung zur Todesstrafe in der Volksrepublik China, die das Max-Planck-Institut in enger Zusammenarbeitet mit der Wuhan University durchführt. Während das Max-PlanckInstitut für die Bevölkerungsbefragung verantwortlich ist, führen die Partner aus Wuhan die parallele Studie über die Einstellungen von Praktikern und Experten im Strafrechtssystem zur Todesstrafe und deren Anwendung durch. Die Bevölkerungs- und die Expertenbefragungen fanden zwischen Ende 2007 und Anfang 2008 statt und werden derzeit ausgewertet.

Die Einstellung der Bevölkerung zur Todesstrafe hat in der kriminalpolitischen und kriminologischen Diskussion seit langem eine wichtige Bedeutung und wird in Meinungsumfragen regelmäßig thematisiert. Aus der abolitionistischen Perspektive stellen die Ergebnisse der Meinungsumfragen eine Herausforderung dar, da die Befürworter der Todesstrafe in vielen Ländern eine Mehrheit bilden. Regierungen können sich daher häufig auf die öffentliche Meinung und traditionelle Strafeinstellungen berufen, wenn sie die Abschaffung der Todesstrafe ablehnen. Das historische Bespiel der Bundesrepublik Deutschland zeigt, dass die öffentliche Meinung eine mehrheitlich ablehnende Haltung zur Todesstrafe erst nach deren Abschaffung entwickelt hat.

Über die Frage der grundsätzlichen Befürwortung oder Gegnerschaft hinaus hat die Einstellungsforschung in vielen westlichen Ländern untersucht, welche Motive bei den Befürwortern der Todesstrafe im Vordergrund stehen, und in welchem Maße die öffentliche Meinung durch wissenschaftliche Erkenntnisse über die Probleme der Todesstrafe (Hinrichtung von Unschuldigen, mangelnde Abschreckungswirkung) beeinflussbar ist. Anhand von Fallbeispielen (Vignetten) wird zudem erkundet, wie sich die abstrakte Befürwortung der Todesstrafe auf die Strafbedürfnisse in konkreten Fällen auswirkt. Da Meinungsbefragungen zur Todesstrafe in China keine Tradition haben, hat das Max-Planck-Institut hier wissenschaftliches Neuland betreten.

Die repräsentative Bevölkerungsbefragung wurde in drei Provinzen (Beijing, Hubei und $\mathrm{Gu}^{-}$ angdong) mit je 1.500 Befragten im Auftrag des Max-Planck-Institutes vom Research Center for Contemporary China (RCCC, Beijing University) zwischen November 2007 und Januar 2008 
durchgeführt. Es handelte sich um eine „face-toface“ Befragung zufällig ausgewählter Personen durch trainierte Interviewer entsprechend wissenschaftlicher Methodenstandards. Die räumliche Auswahl der Stichprobengebiete innerhalb der ausgewählten Gemeinden und die Steuerung der Interviewer vor Ort erfolgte mit Hilfe von GPSEmpfängern nach einem vom RCCC entwickelten Verfahren. Dadurch konnte im Vergleich zu konventionellen Verfahren verbesserte Abbildqualität der Bevölkerungsstichprobe erreicht werden. Die Ausschöpfungsrate liegt mit ca. $69 \%$ höher als in vergleichbaren deutschen Befragungen. Ca. $30 \%$ der Befragten sind nicht offiziell an ihrem Wohnort registriert; damit wurde auch die wichtige Gruppe der Wanderarbeiter erfasst. $14 \%$ der Befragten in Beijing sind Mitglied der Kommunistischen Partei. Die Befragung wurde von den chinesischen Behörden weder genehmigt noch kontrolliert. Die relativ geringen Unterschiede zwischen den drei strukturell sehr unterschiedlichen Provinzen lassen darauf schließen, dass die Ergebnisse auf China insgesamt verallgemeinert werden können. Bei der Interpretation ist jedoch zu bedenken, dass Meinungsbefragungen in China keine Tradition haben und viele, insbesondere „einfache“ Menschen in ländlichen Gebieten und mit geringem Bildungsstatus es nicht gewohnt sind, ihre Meinung frei zu äußern oder überhaupt eine eigene Meinung zu haben.
Die zentralen Befragungsergebnisse zeigen zunächst, dass die Mehrheit der Befragten (74\%) an dem Thema Todesstrafe kein oder nur ein geringes Interesse hat. Die große Mehrheit gibt an, nichts oder nur wenig über die Todesstrafe zu wissen. Dies spiegelt sich auch in einem hohen Anteil von Befragten wider, die viele Fragen gar nicht oder unentschieden beantworten. $80 \%$ Frauen ohne Schulabschluss gehören zu dieser Gruppe der Befragten ohne ausgeprägte persönliche Meinung; am anderen Ende der Bildungsskala, bei Männern wie Frauen mit Hochschulabschluss, liegt dieser Anteil nur bei ca. $25 \%$. Je höher also der Bildungsstatus, desto eher wird eine klare persönliche Meinung zur Todesstrafe vertreten. Diese ist jedoch nicht unbedingt kritischer; mit steigendem Bildungsstatus nehmen sowohl die Befürworter als auch die Gegner der Todesstrafe zu.

Es zeigt sich insgesamt, dass die Mehrheit der Befragten - wie zu erwarten - grundsätzlich für die Todesstrafe ist. Auf die „Standardfrage“ zur Todesstrafe generell antworten $58 \%$ befürwortend, $28 \%$ unentschieden und $14 \%$ ablehnend. Zum Vergleich: In den USA sind aktuell ca. $63 \%$ für und $30 \%$ gegen die Todesstrafe. Wenn jedoch die Frage lautet, ob sie als Mitglied des Volkskongresses einen Antrag auf Abschaffung der Todesstrafe unterstützen würden, lehnen dies nur $31 \%$
Abbildung 1:

Bevorzugte Strafe als schwerste Sanktion für verschiedene Delikte ( $\mathrm{N}=4472$ Befragte)
Sexueller Missbrauch eines Mädchens unter 14 Jahren

Terrorismus

Fälschung von Arzneimitteln

Banknotenfälschung

Diebstahl

Korruption

Vergewaltigung einer erwachsenen Frau

\section{Todesstrafe}

$\square$ lebenslang ohne vorzeit. Entlassung

$\square$ lebenslang mit vorzeit. Entlassung

$\square$ zeitlich befristete Haftstrafe

$\square$ andere Strafe

$\square$ keine Angabe

Unterschlagung

Zuhälterei

Spionage

Raub

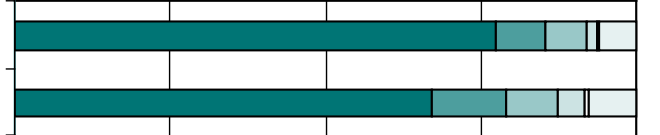

\section{즐}
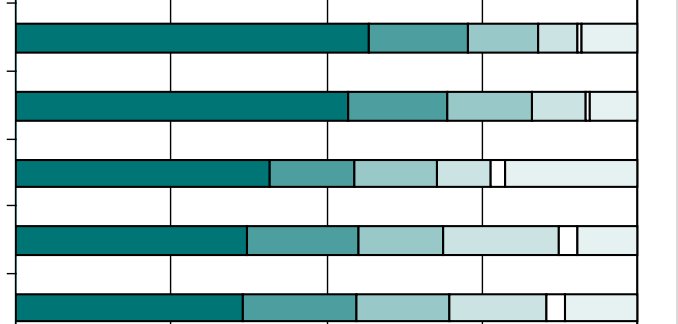

$-$

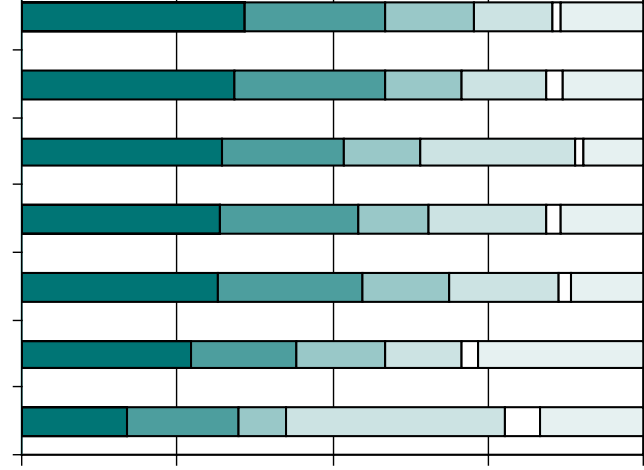

$0 \%$

$50 \%$

$75 \%$ 
Abbildung 2:

Befürwortung der Todesstrafe in einem fiktiven Fall mit vier randomisierten Versionen
Version A: Eine Frau vergiftet ihren Ehemann, der sie seit Langem misshandelt hat. Nach dem Tod ihres Mannes meldet sie das Verbrechen der Polizei.

Version B: Eine Frau vergiftet ihren Ehemann, der sie seit Langem misshandelt hat. Ein Nachbar entdeckte den Tod und meldete das Verbrechen der Polizei.

Version C: Eine Frau vergiftet ihren Ehemann, um mit ihrem Liebhaber zu leben. Nach dem Tod ihres Mannes meldet sie das Verbrechen der Polizei.

Version D: Eine Frau vergiftet ihren Ehemann, um mit ihrem Liebhaber zu leben. Ein Nachbar entdeckte den Tod und meldete das Verbrechen der Polizei.

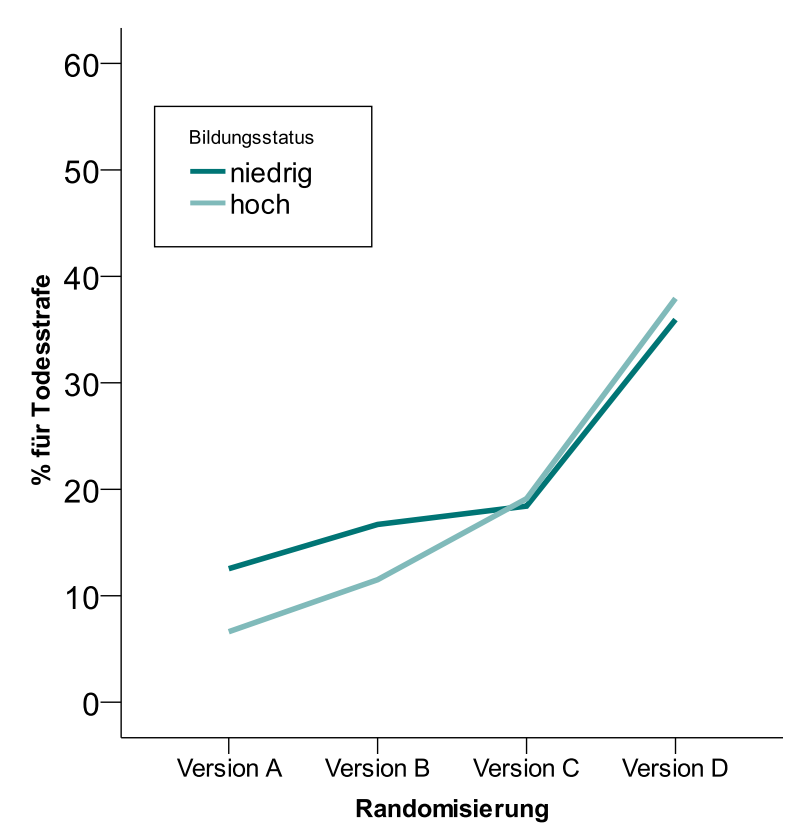

die Unterschiede zwischen den Varianten eindeutig den unterschiedlichen Fallschilderungen zugeschrieben werden können. ohne Möglichkeit einer frühzeitigen Haftentlassung, Entschädigung der Opfer) würden bis zu $70 \%$ der Befragten für die Abschaffung der Todesstrafe stimmen. Daraus kann geschlossen werden, dass die chinesische Bevölkerung durchaus bereit ist, Reforminitiativen der Regierung zu folgen, und eine Berufung der chinesischen Führung auf den „Volkeswillen“ bei der Frage der Todesstrafe nicht gerechtfertigt ist; vielmehr ergibt sich aus diesem Befund ein Gestaltungsspielraum für die politische Führung.

Fragt man nach der bevorzugten schärfsten Strafe für bestimmte Delikte, so ergibt sich nur für vier Delikte eine Mehrheit für die Todesstrafe (Abb. 1). Aus einer Liste von 14 Verbrechen erreichen nur Mord, Körperverletzung mit Todesfolge, Drogenhandel und sexueller Missbrauch von unter 14-jährigen Mädchen Zustimmungsraten von mehr als $50 \%$ für die Todesstrafe als schärfste Sanktion für die schlimmsten Fälle. Im chinesischen Strafgesetzbuch ist die Todesstrafe gegenwärtig jedoch für 68 Delikte vorgesehen.

Wenn die Befragten mit konkreten Fallbespielen (Vignetten) konfrontiert werden, sinkt die Zustimmung zur Todesstrafe deutlich gegenüber der abstrakten Frage. Bei der Befragungstechnik der „randomized vignettes“ wird jede Vignette in vier jeweils unterschiedlichen Varianten vorgestellt, die sich in der Deliktschwere oder dem Verhalten der Täter unterscheiden. Jeder Befragte erhält zufällig nur eine der vier Varianten vorgelegt, sodass
Nur in den schlimmsten Fällen von Tötungsdelikten erreicht die Todesstrafe (mit sofortiger Exekution) Zustimmungsraten von etwas mehr als $50 \%$; sonst liegt sie weit darunter. In Abb. 2 dokumentieren wir die Ergebnisse für eine der vier Vignetten. Obschon der geschilderte Fall in Version D einen Mord aus niederen Beweggründen darstellt, entscheiden sich auch hier nur rund $40 \%$ der Befragten für die Todesstrafe. In den anderen Versionen liegt dieser Anteil noch wesentlich darunter. Zum Beispiel sprechen sich im Falle einer Drogendealerin, die $1 \mathrm{~kg}$ Heroin schmuggelt, nur $10 \%$ der Befragten für die Todesstrafe aus. Das Bildungsniveau der Befragten hat auf diese Sanktionswahl keinen wesentlichen Einfluss. Das Ergebnis zeigt, dass die chinesischen Befragten eher zurückhaltend bei der Befürwortung der Todesstrafe sind und ihre Strafpräferenzen unterschiedlichen Fallkonstellationen sehr bewusst anpassen.

Diese Ergebnisse lassen sich insgesamt als eine Unterstützung von Bestrebungen zumindest zur Zurückdrängung der Todesstrafe in der chinesischen Strafrechtspraxis interpretieren.

Weitere Ergebnisse betreffen dann die nachfolgenden Einstellungsmuster:

- Bereitschaft zur Meinungsänderung: Viele Befürworter wären bereit, ihre Meinung angesichts wissenschaftlicher Erkenntnisse über die Hin- 
richtung von Unschuldigen zu ändern; eine wissenschaftliche Widerlegung der abschreckenden Wirkung würde sie demgegenüber weniger beeinflussen.

- Zahl der Exekutionen: Nur 16\% antworten zustimmend, aber 54\% unentschieden auf die Frage, ob ihres Erachtens in China zu viele Hinrichtungen stattfinden. $64 \%$ der Befragten $(68 \%$ der KP-Mitglieder) befürworten eine Veröffentlichung der jährlichen Zahl der Exekutionen.

- Ungleiche Anwendung: Die meisten Befragten (69\%) sind davon überzeugt, dass die Todesstrafe ungleich in Hinblick auf die soziale Schichtzugehörigkeit der Angeklagten angewendet wird. Unter den KP-Mitgliedern sind dies sogar $76 \%$.

- Rechtsstaatliche Anwendung: Eine große Mehrheit unterstützt die rechtsstaatliche Anwendung der Todesstrafe (zweifelsfreie Feststellung der Schuld, faires Gerichtsverfahren etc.)

- Beeinflussung von außen: Eine Beschränkung der Anwendung der Todesstrafe auf die „schwersten Verbrechen" im Sinne der UN-Resolution wird von $49 \%$ befürwortet (von $70 \%$ der Befragten mit Hochschulabschluss); allerdings ist der Begriff der „schwersten Verbrechen“ nicht definiert.
Eine Mehrheit lehnt die Orientierung am Beispiel anderer Länder hinsichtlich der Abschaffung der Todesstrafe $(55 \%)$ und eine Beschleunigung der Politik hin zu einer Abschaffung $(53 \%)$ ab.

Die Befragungsergebnisse geben trotz der fehlenden Tradition einer offenen und unabhängigen Meinungsbildung in China wichtige Hinweise und realistische Instrumente für eine Reform der Anwendung der Todesstrafe unterhalb des Endziels ihrer vollständigen Abschaffung. Forderungen nach einer deutlichen Beschränkung ihrer Anwendung, nach Sicherstellung rechtsstaatlicher Prinzipien bei der Anwendung und nach einer insgesamt offenen, transparenten Berichterstattung lassen sich direkt aus diesen Befragungsergebnissen ableiten. Vor dem Hintergrund der historischen Erfahrungen anderer Länder kann zudem argumentiert werden, dass der angebliche Volkeswille auch in China kein maßgeblicher Hinderungsgrund für eine Abschaffung der Todesstrafe ist.

Dietrich Oberwittler, Shenghui Qi 


\title{
G. Risiko und Risikokontrolle
}

\author{
Forschungsschwerpunkt Sexualstraftäter
}

Kriminologische

Forschungsabteilung

Forschungsschwerpunkt: Gefährliche Straftäter

Im Fokus des Forschungsschwerpunkts „Gefährliche Straftäter“ stehen vor allem Sexualstraftäter, die als eine besondere Herausforderung und herausragende Gefahr für die Gesellschaft wahrgenommen werden. Fragen des Verlaufs, der Behandlung, der Prognose und des Rückfalls sind nach wie vor nicht hinreichend beantwortet. Die Forschungstätigkeit im Berichtszeitraum 2006/2007 knüpft dabei an bisherige Untersuchungen zu Sexualstraftätern an. Fragestellungen der Gefährlichkeit werden in Längsschnittuntersuchungen aufgegriffen bzw. fortgeführt, so z.B. in der Evaluationsstudie der sozialtherapeutischen Abteilungen des Freistaates Sachsen oder in der im Rahmen der Kohortenstudie untersuchten Fragestellung nach Prävalenz, Inzidenz und Verlauf von Sexualkriminalität im Lebenslängsschnitt. Eine Studie zur Legalbewährung von Sicherungsverwahrten und gefährlichen Straftätern konzentriert sich auf den Beitrag, den die in den letzten Jahren in mehreren Schritten ausgeweitete Sicherungsverwahrung zur Prävention schwerer Kriminalität, insbesondere Gewaltkriminalität, zu leisten vermag. Diese umfassenden, längsschnittlich angelegten Projekte werden flankiert von Studien, die einzelne Fragestellungen verfolgen.

Das Projekt zur Evaluation sozialtherapeutischer Abteilungen des Freistaates Sachsen geht einerseits der Frage nach, wie wirksame, auch tätersubgruppenspezifische Behandlung gestaltet sein muss (was verhindert den Rückfall in schwere Kriminalität?) und untersucht andererseits rückfallrelevante Faktoren der Sexualdelinquenz. Hierbei werden Insassen der Justizvollzugsanstalten Sachsens zu vier Erhebungszeitpunkten befragt, um im Rahmen einer prä-post-Messung inhaltsspezifische Effekte der sozialtherapeutischen Behandlung zu identifizieren. Zum Jahresende 2007 lagen die Daten von ca. 170 befragten Insassen zum Befragungszeitpunkt $t_{1}$ vor, der im Beginn der Haftphase liegt. Die Daten wurden daraufhin untersucht, ob sich die im Rahmen einer früheren am Max-Planck-Institut durchgeführten Studie identifizierten Subtypen von Sexualstraftätern (Wößner 2006) auch in dieser Stichprobe replizieren lassen und ob auch bei den Gewaltstraftätern (also Inhaftierte, die nicht wegen eines Sexualdeliktes verurteilt wurden) indikationsrelevante subgruppenspezifische Merkmalsgefüge beobachtet werden können. Denn diese Tätergruppe wird - wie auch an der Entwicklung der Belegungszahlen der Sozialtherapeutischen Abteilungen zu sehen ist, nahm der Anteil der Gewaltstraftäter in der Sozialtherapie zugunsten der Sexualstraftäter über die letzten Jahre hinweg kontinuierlich ab seit Inkrafttreten des Gesetzes zur Bekämpfung von Sexualdelikten und anderen gefährlichen Straftaten (1998) zunehmend vernachlässigt. Es lassen sich in diesem Untersuchungsschritt fünf Subgruppen von Sexualstraftätern und vier Subgruppen von Gewaltstraftätern identifizieren. Bei den Gewaltstraftätern ist vor allem die Differenzierung von drei Hauptgruppen interessant: (i) sozial und psychopathologisch unauffällige, oft im Zusammenhang mit einer Lebenskrise gewalttätig werdende Täter, die kaum Vorstrafen und vor allem fremde Opfer mit einem breiten Deliktschwerpunkt aufweisen, (ii) eine generell dissoziale, gewalttätige auf Raubdelikte fokussierte Tätergruppe, die nur geringfügig mit Suchtproblematik belastet ist und schließlich (iii) bei der Tat zu extremer Gewalt neigende Täter mit einer ausgeprägten Suchtproblematik. In der Stichprobe der Sexualstraftäter lässt sich, wie bereits in der früheren Studie, in der vorliegenden Studie eine Tätergruppe identifizieren, die sich durch ein durchgängiges dissoziales Verhaltensmuster auszeichnet. Ebenfalls wieder zu finden ist eine Gruppe sozial unauffälliger Missbrauchstäter mit ausgeprägter Selbstwertproblematik und selbstunsicherer Persönlichkeitsakzentuierung, die allerdings im Gegensatz zu der in der früheren Studie erhobenen vergleichbaren Gruppe zudem erhebliche Alkoholprobleme aufweist. Daneben ergeben sich auch neue Subgruppen. Die Divergenzen zwischen den Zwischenergebnissen der aktuellen 


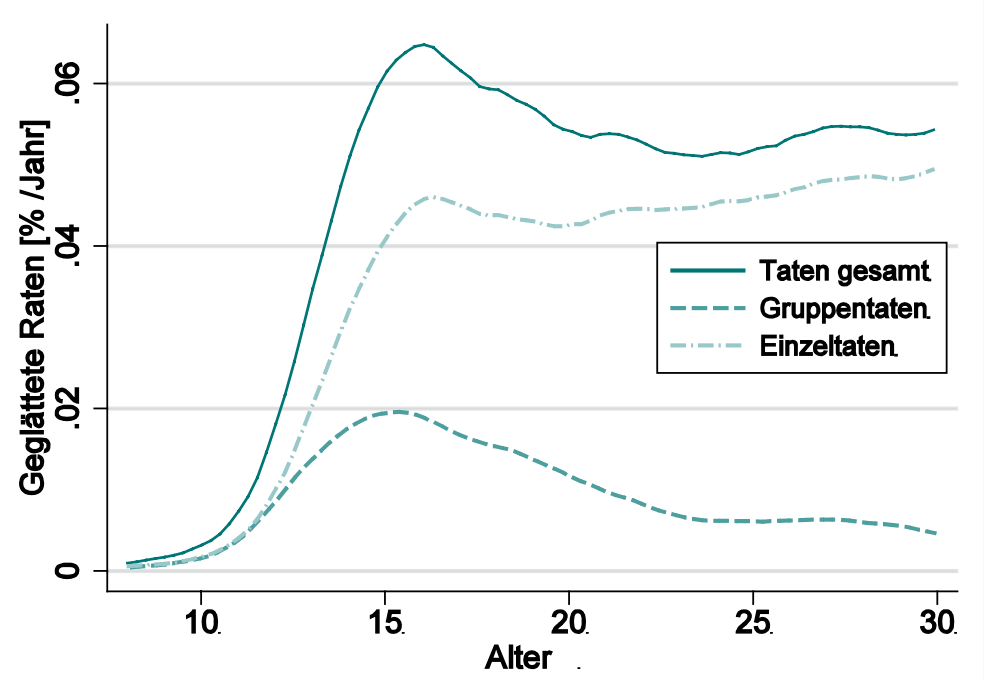

Studie und der früheren Untersuchung dürften damit erklärt werden können, dass im Rahmen der laufenden Untersuchung versucht wurde, die Typisierung anhand standardisierter Testverfahren zu replizieren, und dass sich die Stichprobe der aktuellen Studie maßgeblich von der Stichprobe der früheren Untersuchung unterscheidet (in der früheren Studie wurden auch im Maßregelvollzug untergebrachte Sexualstraftäter exploriert). Die Frage, ob die hier angedeuteten, noch feineren Differenzierungen auch für Fragen der Behandlung relevant sind, muss in weiterführenden Analysen untersucht werden. Dass eine subgruppenspezifischere Herangehensweise allerdings notwendig ist, zeigt die vergleichende Querschnittsanalyse von Gewalt- und Sexualstraftätern bezüglich der untersuchten Variablen: Nur für einzelne Aspekte ließ sich aufgrund statistisch signifikanter Ergebnisse die Aussage ableiten, dass sich Gewalt- und Sexualstraftäter generell voneinander unterscheiden. Im Bereich der Selbstkontrolle sind die Gewaltstraftäter ich-bezogener und risikobereiter als Sexualstraftäter (FES-K, Grasmick et al. 1993) und die Gewaltstraftäter erreichen einen signifikant höheren Wert auf einer Skala zur Erfassung der Aggressivität (FPI-R, Fahrenberg et al. 2001). Dies deutet darauf hin, dass Unterschiede in der Gesamtstichprobe „verschwinden“ und erst durch eine differenzierte Betrachtung einzelner Subgruppen in Erscheinung treten.

Auch mit der Analyse der Daten der Freiburger Kohortenstudie lässt sich zeigen, dass es einer (typen-)spezifischen Differenzierung der Sexualstraftäter bedarf. Dies kann vor allem an unterschiedlichen Verläufen und Tatbegehungsmustern festgemacht werden. Es zeigte sich beispielsweise, dass, wie auch bei anderen Delikten, die Tatbe- gehung in einer Gruppe überwiegend im Jugendalter auftritt. Aus Abbildung 1 ergibt sich, dass im Jugendalter ca. ein Drittel aller Sexualdelikte in einer Gruppe begangen wurden. Bei den über 25-Jährigen sinkt der Anteil der Gruppentaten auf unter $10 \%$. Nach Tatbeständen differenziert zeigt sich, dass ca. die Hälfte aller Vergewaltigungen/ sexuellen Nötigungen von unter 19-Jährigen in einer Gruppe begangen werden, während es beim sexuellen Missbrauch knapp über $10 \%$ sind. Interessanterweise hängt die Form der Tatbegehung in einer Gruppe oder einzeln mit den Rückfallraten zusammen. So werden von den Einzeltätern innerhalb von fünf Jahren nach einer justiziellen Erledigung wegen eines Sexualdelikts ca. $5 \%$ rückfällig. Bei den Gruppentätern tritt ein Rückfall bei etwa $2 \%$ auf. Von den jugendlichen Tätern sind ca. zwei Drittel den Opfern bekannt, bei den über 18-Jährigen sinkt der Anteil bekannter und verwandter Opfer auf gut $50 \%$. Bei einer genaueren Analyse der Verurteilungen aufgrund von sexueller Nötigung oder Vergewaltigung zeigen die Daten der Freiburger Kohortenstudie erstens, dass die Häufigkeit von Registrierungen nach einem Anstieg zu Beginn der Pubertät mit zunehmenden Alter kaum mehr variiert und zweitens, dass es sich bei den Registrierungen aufgrund dieser Delikte zu $80 \%$ um Erstregistrierungen handelt. Eine einschlägige Vorstrafenhistorie geht somit in den wenigsten Fällen voraus. Gleichwohl weisen wegen einer Vergewaltigung/Nötigung Verurteilte sowohl vor als auch nach der Sexualstraftat durchaus Registrierungen mit anderen Delikten auf (Albrecht \& Grundies 2007).

Es ist fraglich, ob der konstante Altersverlauf im Bereich der Vergewaltigungsdelikte tatsächlich mit einer konstant verlaufenden Prävalenz ent-
Abbildung 1:

Häufigkeit von Sexualdelikten (Vergewaltigung/sexuelle Nötigung und sexueller Missbrauch) differenziert nach der Tatbegehung in der Gruppe oder einzeln (deutsche Männer, Baden-Württemberg) 
sprechender Delikte einhergeht. Vielmehr ist zu vermuten, dass sich in den letzten Jahren auch eine zunehmende Bereitschaft zur Anzeige sexueller Gewaltdelikte entwickelt hat, evtl. sogar ganz dezidiert im Bereich häuslicher und familiärer Gewalt.

Sowohl die Ergebnisse der Kohortenstudie als auch die Zwischenergebnisse der Evaluationsstudie der sozialtherapeutischen Abteilungen des Freistaates Sachsen zeigen, dass es nur eine kleine Gruppe hochspezialisierter und somit immer wieder einschlägig rückfallgefährdeter Sexualstraftäter gibt. Dass die Rechts- und Sanktionswirklichkeit indes unter einem anderen Vorzeichen steht, konnte die im Berichtszeitraum abgeschlossene Studie zur Legalbewährung von Sicherungsverwahrten und gefährlichen Straftätern zeigen. Interessant sind deren Ergebnisse vor allem, weil anhand einer Kontrollgruppe von 162 Probanden, für die trotz des Vorliegens der formalen Voraussetzungen die Sicherungsverwahrung nicht angeordnet wurde, eine vergleichende Analyse der Rückfallkriminalität mit 286 Sicherungsverwahrten durchgeführt wurde. Da diese Gruppe nach durchschnittlich 5,7 Jahren entlassen wurde, war ein Vergleich der Wiederverurteilungen der Probanden der beiden Gruppen nach jeweils ca. 5 Jahren möglich. Zwar waren es vor allem Sexualstraftäter, die erneut Sexualdelikte begangen hatten, dies traf jedoch nur auf rund ein Drittel der Probanden zu, bei denen keine Sicherungsverwahrung anhängig war. Außerdem war der Anteil an schwerer Delinquenz bei den rückfälligen Nicht-Sicherungsverwahrten mit $19 \%$ relativ niedrig. In beiden Gruppen wurde nur ein Drittel der Sexualstraftäter wieder einschlägig rückfällig, bei den Raubtätern waren es $26 \%$.

In einem weiteren Forschungsprojekt, in dessen Mittelpunkt die Validierung jugendspezifischer Prognoseinstrumente steht, wurden jeweils 147 jugendliche Sexual- und Gewaltstraftäter retrospektiv untersucht. Anders als bei den Erwachsenen der Sozialtherapie-Untersuchung konnten in dieser Studie Unterschiede zwischen jugendlichen Gewalt- und Sexualstraftätern in ausgewählten Merkmalsbereichen identifiziert werden. Welche dieser Unterscheidungsmerkmale auch zu einer längerfristigen Prognose „chronisch“ bzw. einschlägig rückfälliger Täter dienen können bzw. ob dies überhaupt möglich ist, wird in weiteren Analysen noch zu klären sein. Denn wenn den Ergebnissen der Kohortenstudie folgend die meisten Registrierungen eines Sexualdelikts (Vergewaltigung) im Erwachsenenalter Erstregistrierungen sind, so ist zu klären, welche bereits im Jugendalter mit welchen spezifischen Merkmalen auffällig werdenden Sexualstraftäter später in die relativ kleine Gruppe derjenigen fällt, die immer wieder mit einem Sexualdelikt rezidivieren. Zusammenfassend lässt sich konstatieren, dass sich junge Sexual- und Gewaltstraftäter sowohl hinsichtlich der strafrechtlichen Vorbelastung als auch einiger biographischer und psychopathologischer Besonderheiten unterschieden.

In der Gesamtschau der einzelnen Zwischenergebnisse zeigt sich, dass es zwar vor allem Sexualstraftäter sind, die im Falle eines Rückfalls wieder zu Sexualdelikten neigen. Jedoch macht dies nur einen ganz geringen Anteil der (Rückfall-)Straftaten aus. Die Frage nach eindeutigen Kriterien für die Identifizierung chronischer Sexualstraftäter muss ebenso wie die Frage, unter welchen (institutionellen, psychosozialen, biographischen und persönlichkeitspsychologischen) Voraussetzungen eine (sozialtherapeutische) Behandlung präventiv wirken kann, weiter abgeklärt werden. Zwar mag es in einzelnen, eindeutigen Fällen leicht erscheinen, diese als rückfallgefährdet zu identifizieren, doch besteht immer noch erheblicher Bedarf an Wissen darüber, wie falsch negative und falsch positive Prognosen minimiert werden können. Vor allem der Befund der Kohortenstudie, dass bei den Vergewaltigungen der Hauptanteil der Registrierungen durch Erstregistrierungen zu erklären ist, weist darauf hin, dass eine Formel im Sinne „die jugendlichen Sexualstraftäter von heute sind die Sexualstraftäter von morgen“ zu kurz greift. Die fortlaufenden empirischen Untersuchungen im Rahmen des Forschungsschwerpunkts werden hier zu weiterer Klärung und Wissensakquirierung beitragen.

Gunda Wößner, Volker Grundies, Jörg Kinzig, Carolin Quenzer, Sonja Brauner 


\title{
H. Informationsgesellschaft
}

\author{
Die Überwachung der Telekommunikation
}

Vor dem Hintergrund einer zunehmenden praktischen Bedeutung verdeckter Ermittlungsmaßnahmen, ihres Potenzials für grundlegende Veränderungen des Strafverfahrens und des damit zusammenhängenden Bedeutungsgewinns präventiver Zielsetzungen konzentriert sich die empirische Strafverfahrensforschung der Kriminologischen Abteilung in einem besonderen Forschungsschwerpunkt auf die Überwachung der Telekommunikation und andere verdeckte Ermittlungsmethoden. Die Evaluationsstudie zu Rechtswirklichkeit und Effizienz der Überwachung der Telekommunikation gem. $\$ \S 100$ a und b StPO (vgl. Tätigkeitsbericht 2002/03, S. 146ff., Forschungsbericht 2004/05, S. 17 lff.) und weitere Untersuchungen zur akustischen Wohnraumüberwachung und zur Rasterfahndung (vgl. Forschungsbericht 2004/05, S. 180f. u. 182f.) werden nunmehr ergänzt um eine Untersuchung zur Überwachung der Telekommunikationsverbindungsdaten (Verkehrsdaten) gem. $\$ \S 100 \mathrm{~g}$ und h StPO. Die Untersuchung steht auch im Zusammenhang mit der zum 1. Januar 2008 in Kraft getretenen Vorratsspeicherung von Telekommunikationsverkehrsdaten, die wiederum auf einer Richtlinie der Europäischen Union beruht. Die zentralen Fragestellungen beziehen sich auf den Beitrag von Verkehrsdaten für den Erfolg von strafrechtlichen Ermittlungen sowie auf den Bedarf an retrospektiver Erfassung von Verkehrsdaten der Telekommunikation. Der Forschungsbericht ist online auf den Websites des MaxPlanck-Instituts sowie des Bundesministeriums der Justiz verfügbar ${ }^{1}$. Eine gedruckte Version wird ab Sommer 2008 in der Reihe Kriminologische Forschungsberichte aus dem Max-Planck-Institut als Band K 139 verfügbar sein. Das Gutachten ist darüber hinaus in einer inhaltsgleichen Volltextfassung als BT-Drucksache 16/8434 erschienen.

Der Zugriff auf Telekommunikationsverkehrsdaten ist ein Ermittlungsinstrument, dem in neuerer Zeit neben der Überwachung der Kommunikationsinhalte eine zunehmende Bedeutung zugeordnet wird. Eine zentrale Rolle spielen Verbindungsdaten, insbesondere für die Bekämpfung der Datennetzkriminalität. Jedoch dürften Verbindungsdaten angesichts der sich dramatisch verdichtenden digitalen Kommunikation für Ermittlungen in fast allen Bereichen der Kriminalität von Belang sein. Spektakuläre Fälle der Kinderpornographie, in denen teilweise mehrere Zehntausend Tatverdächtige weltweit über Internetverbindungsdaten identifiziert wurden, unterstreichen die Relevanz der Verkehrsdatenabfrage. Sie dienen zur Bestimmung des Aufenthaltsortes von Personen, zur Beweisermittlung oder zur Offenlegung von Täterstrukturen und Netzwerken der Transaktionskriminalität. Verkehrsdaten werden von der Praxis weithin - wie nicht zuletzt die Richtlinie der Europäischen Union für die Vorratsspeicherung (2006/24/EG) ${ }^{2}$ und das darauf bezogene Bundesgesetz zur Neuregelung der Telekommunikationsüberwachung und anderer verdeckter Ermittlungsmaßnahmen vom 21. Dezember $2007^{3}$ demonstrieren - als unver- zichtbare Informationsquelle angesehen. Mit der Abfrage von Verkehrsdaten kann grundsätzlich in Erfahrung gebracht werden, wer mit wem wann telefoniert hat, welche Internetseiten angewählt wurden, wo sich ein Kommunikationsteilnehmer zu einem bestimmten Zeitpunkt befand und welche (mobilen) Kommunikationsmittel zu welchen Zeiten in einer Funkzelle angemeldet waren.

Die Untersuchung beruht auf einem Mehrmethodenzugang. Neben der Analyse von Strafakten wurden Befragungen sowie Experteninterviews durchgeführt. Im Übrigen konnte auf die bei verschiedenen Telekommunikationsprovidern anfallenden Daten zu Verkehrsdatenabfragen zurückgegriffen werden.

Aussagen über die Gesamtzahl der Beschlüsse oder der Abfragen zu unterschiedlichen Bereichen von Verkehrsdaten lassen sich auf der Grundlage offizieller Statistiken ebenso wenig treffen wie zu den Entwicklungen in diesem Bereich. Zur Schätzung von Umfang und Entwicklung der Verkehrsdatenabfragen wurden die bei Telekommunikationsanbietern verfügbaren internen Statistiken einer Auswertung unterzogen. Aus diesen
Kriminologische Forschungsabteilung

Forschungsschwerpunkt: Strafverfahren und Sanktionen im Wandel 
Abbildung 1:

Entwicklung der Verkehrsdatenabfragen 2000 - 2005 (geschätzt)

Abbildung 2:

Entwicklung der Verkehrsdatenabfragen im Mobilfunkbereich

(1 Unternehmen, 31,6 Millionen Kunden)

\section{Abbildung 3:}

Arten der Verkehrsdatenabfragen in den untersuchten Beschlïssen
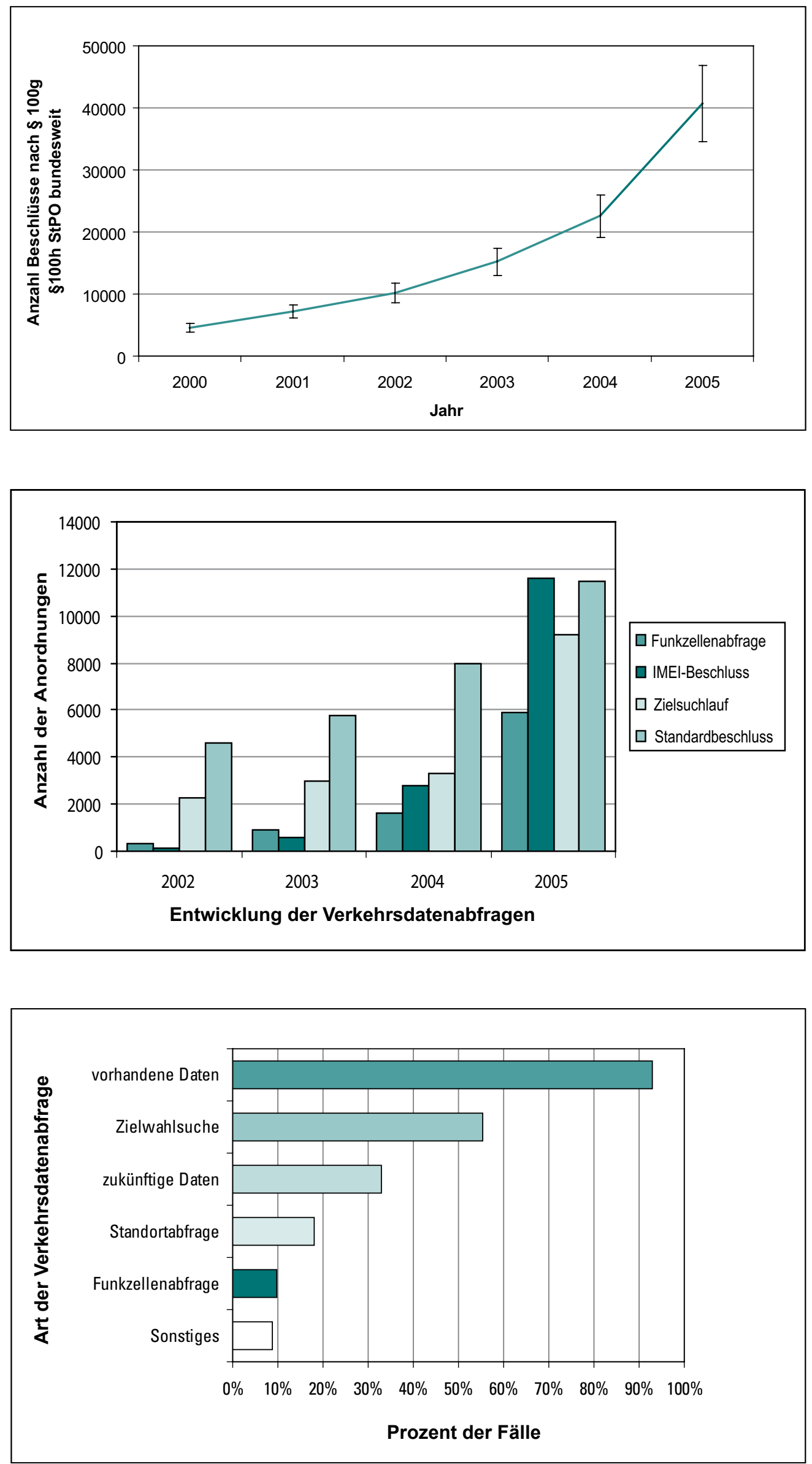
Daten können Informationen zum Umfang und zur Struktur der Verkehrsdatenabfrage sowie zu ihrer Entwicklung zwischen 2002 und 2005 entnommen werden.

Der in Abbildung 1 enthaltene mittlere Wert der Hochrechnungen repräsentiert die bestmögliche Schätzung der Anzahl der Beschlüsse nach $\$ \$ 100 \mathrm{~g}, 100 \mathrm{~h}$ StPO. Er ist zusammen mit der Fehlerspanne dargestellt. Danach lag die Zahl der Verkehrsdatenabfragen im Jahr 2005 bei etwa 40.000 (ohne Abfrage der Zuordnung von dynamischen IP-Adressen zu einem Anschlussinhaber bzw. Internetnutzer). Die Tendenz ist, wie ersichtlich, stark ansteigend. Im direkten Vergleich mit den Daten aus der Untersuchung zur Telekommunikationsüberwachung gem. $\$ \S 100 \mathrm{a}, 100 \mathrm{~b}$ StPO wurde demnach in 2005 erstmals etwa gleich häufig die Abfrage von Verkehrsdaten und Inhaltsüberwachungen angeordnet (Telekommunikationsüberwachung ca. 42.500 Beschlüsse inkl. Verlängerungen und ca. 40.700 Beschlüsse zu Verkehrsdatenabfragen). In den Jahren davor lag die Abfrage von Verkehrsdaten noch deutlich unter der Anzahl von Inhaltsüberwachungen (2004: Telekommunikationsüberwachung 34.400, Verkehrsdaten 22.600; 2003: Telekommunikationsüberwachung 29.430, Verkehrsdaten 15.200 und 2002: Telekommunikationsüberwachung 26.200, Verkehrsdaten 10.200).

Für die Entwicklung der verschiedenen Arten der Verkehrsdatenüberwachung kann auf detaillierte Angaben einzelner Telekommunikationsunternehmen zurückgegriffen werden. Wie sich $A b$ bildung 2 entnehmen lässt, stieg die Zahl der Beschlüsse, die eine Abfrage zu einer sogenannten IMEI-Nummer (International Mobile Equipment Identity) beinhalteten, etwa um das Vierfache. Die angeordneten Zielwahlsuchen, die auch als Zielsuchläufe bezeichnet werden, haben sich im gleichen Zeitraum verdreifacht.

Dem entspricht auch das Bild der Entwicklung der Anordnungen nach $\$ \$ 100 \mathrm{~g}, 100 \mathrm{~h}$ StPO im Festnetzbereich. Hier ist ein kontinuierlicher Anstieg der Zielwahlsuchen zu bemerken, während die Abfrage von abgehenden und zukünftigen Verkehrsdaten seit 2003 auf gleichem Niveau bleibt. Ein extremer Anstieg fällt bei der Abfrage von sogenannten IP-Adressen (Internet Protocol Address) im Vergleich von 2004 und 2005 auf. Während 2004 noch Daten zu etwa 6.300 IP-Adressen abgefragt wurden, stiegen diese Anordnungen 2005 auf 75.500. Dies hängt mit großflächigen Ermittlungen im Bereich der Urheberrechtsverlet- zungen (illegale Downloads) zusammen. Allein im Bereich der Staatsanwaltschaft Karlsruhe gingen 2005 etwa 20.000 Anzeigen Urheberrechtsverletzungen betreffend ein; das ist ein Fallbereich, der mittlerweile bei vielen Staatsanwaltschaften zumeist ohne Veranlassung konkreter Ermittlungsmaßnahmen eingestellt wird.

Das maßgebliche Deliktsspektrum der Verkehrsdatenabfrage wird nach den Ergebnissen der Aktenanalyse durch verschiedene Faktoren mitbestimmt. Zum einen unterscheiden sich die zugrunde liegenden Straftaten danach, ob Mobiloder Festnetzanschlüsse überwacht werden. So sind die Überwachungen im Festnetzbereich wesentlich durch den Betrug bestimmt. Dabei spielen Betrugshandlungen, die über eine telefonische Kontaktaufnahme vorbereitet werden, eine beachtliche Rolle (insbesondere der sogenannte Enkeltrick). Von Bedeutung sind ferner Raub und Mord sowie Betäubungsmitteldelikte. Im Mobilfunkbereich dominieren als Anlassdelikte neben den Straftaten aus dem Betäubungsmittelbereich hingegen Raub und Diebstahlsdelikte. Zweites wesentliches Unterscheidungskriterium ist sodann die Art der Verkehrsdatenabfrage. So konzentriert sich beispielsweise die Abfrage von IP-Adressen auf Betrugsdelikte sowie die Bereiche Kinderpornographie und Urheberrechtsverletzung. Bei der Funkzellenabfrage geht es hingegen sehr häufig um Raub- und Diebstahlsdelikte, darunter oftmals auch um die Aufklärung des Verbleibs von gestohlenen oder geraubten Mobiltelefonen. Hier wie bei den Straftaten, die mittels Endeinrichtung begangen werden, bietet die Verkehrsdatenabfrage oftmals überhaupt den einzigen plausiblen Ermittlungsansatz. Insgesamt spricht die Verteilung der Straftatbestände dafür, dass sich die Verkehrsdatenabfrage auf ein weiteres Deliktsspektrum bezieht und nicht die bei der Telekommunikationsüberwachung festgestellten Konzentrationen auf Betäubungsmittel- und andere Transaktionsdelikte aufweist. Neben der Deliktsphänomenologie ergibt sich im Übrigen auch aus den Ergebnissen der Analysen zur Sanktionsstruktur, dass ein bedeutsamer Anteil der Verfahren allenfalls der mittleren Kriminalität zuzuordnen ist.

Ganz überwiegend wurden in den untersuchten Beschlüssen, wie aus Abbildung 3 ersichtlich, bereits gespeicherte Daten abgefragt (93\%). Die Zielwahlsuche wurde in $55 \%$ der Beschlüsse angeordnet. In die Zukunft gerichtet waren $33 \%$ der Abfragen. Standortabfragen (18\%) wurden etwa doppelt so häufig angeordnet wie Funkzellenabfragen (10\% der Fälle). Bei den sonstigen Ab- 
Abbildung 4:

Dauer der Datenerhebung bei den verschiedenen Abfragearten fragen $(4 \%)$ handelte es sich insbesondere um Abfragen der Rufnummer zur IMEI-Nummer, um Auskünfte zur benutzten SIM-Karte sowie um Bestandsdaten. Sehr aufschlussreich sind schließlich die Analyseergebnisse zur Dauer der Verkehrsdatenüberwachung. Auch hier ergeben sich deutliche Unterschiede entlang der Art der Abfrage. Diese werden aus Abbildung 4 deutlich. Während Zielwahlsuchen (B) und Funkzellenabfragen (C) den Schwerpunkt im Stunden- und Minutenbereich haben, wird die Abfrage der von einem überwachten Anschluss abgehenden Verbindungen (A/B) gezielt auf einen längeren Zeitraum hin eingesetzt. Hier liegt der Ermittlungsansatz auf einer breit angelegten Erfassung der Kommunikationsverkehrsdaten eines Tatverdächtigen. Der Schwerpunkt liegt bei zwei bis drei Monaten.

Auf der Basis der Untersuchungsergebnisse erscheint die Verkehrsdatenabfrage insgesamt als eine Maßnahme, die ganz überwiegend im Anfangsstadium des Ermittlungsverfahrens eingesetzt wird. In zwei Drittel der Verfahren war sie auch der Anlass für die erste Einschaltung der Staatsanwaltschaft. Kombinationen mit anderen Ermittlungsmaßnahmen erfolgen vor allem mit der Telekommunikationsüberwachung, aber auch mit der Durchsuchung und der Beschlagnahme.
Telekommunikationsüberwachung und Verkehrsdatenabfrage werden kombiniert angewendet und im Verhältnis zueinander als Ergänzung angesehen. Die Verkehrsdatenabfrage dient dabei häufig der Vorbereitung der Telekommunikationsüberwachung durch Feststellung von Anschlussnummern, für die dann die Telekommunikationsüberwachung geschaltet wird, oder der Identifizierung von Gesprächsteilnehmern. Sie wird gleichzeitig mit der Telekommunikationsüberwachung angeordnet, wenn die Abfrage gespeicherter Daten und die gleichzeitige Anordnung der Telekommunikationsüberwachung für die Zukunft zu einem umfassenden Bild der Kommunikationsmuster führen soll. Hier gilt die Verkehrsdatenabfrage bei den Praktikern aus den Ermittlungsbehörden als sehr ökonomische Maßnahme. Im Übrigen wird der Verkehrsdatenabfrage vor allem die Funktion zugeordnet, erste Ermittlungsansätze zu gewinnen, um dann andere Maßnahmen durchführen zu können. Die Verkehrsdatenabfrage ist demnach - anders als die auf Gesprächsinhalte bezogene Telekommunikationsüberwachung nicht das „letzte“ Mittel, sondern, umgekehrt, das „erste“ Mittel. Anders ist dies nur in Verfahren, in denen die Verkehrsdatenabfrage die einzige erfolgversprechende Ermittlungsmaßnahme darstellt. Zu dem Gesamtbild über den Einsatz der Verkehrsdatenabfrage passt im Übrigen der in den

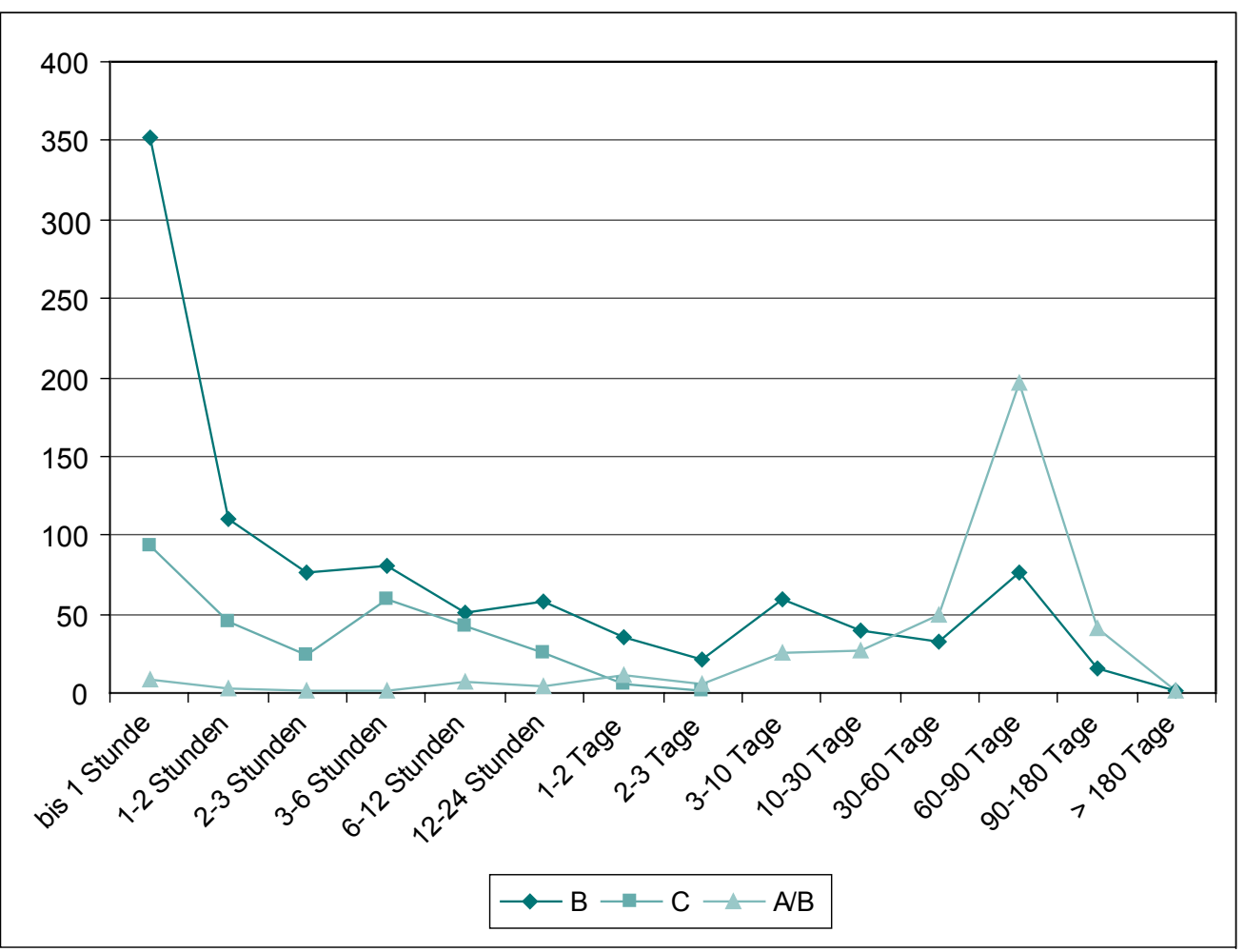


Befragungen ermittelte Befund, dass diese von Praktikern im Vergleich zu konventionellen wie zu anderen verdeckten Ermittlungsmaßnahmen tendenziell als weniger belastend eingeordnet wird.

Alle Untersuchungsergebnisse basieren freilich auf der Rechtslage, wie sie sich in Deutschland vor Einführung der Vorratsdatenspeicherung dargestellt hat. Insoweit orientiert sich die Praxis maßgeblich an den verfügbaren Datenbeständen. Diese Verfügbarkeit war bis dahin weithin von der internen Zweckbestimmung bei den Telekommunikationsunternehmen, insbesondere der Rechnungsstellung, determiniert. Beeinflusst werden Speicher- bzw. Löschpraktiken im Übrigen durch datenschutzrechtliche Vorgaben. Dies hatte in der
Vergangenheit etwa zur Konsequenz, dass aussagefähige Verbindungsdaten bei Kunden mit Prepaid-Karten und Nutzern von sogenannten Flatrates praktisch nicht verfügbar waren. $\mathrm{Ob}$, und gegebenenfalls wie, sich die Überwachungspraxis unter den Bedingungen der nunmehr geltenden sechsmonatigen Vorratsdatenspeicherung - die sich im Gegensatz zur bisherigen Praxis an den Interessen der Strafverfolgung orientieren soll verändern wird, wird Gegenstand einer Followup-Studie zur Verkehrsdatenüberwachung bilden, deren Abschluss bis Ende 2010 geplant ist.

Hans-Jörg Albrecht, Michael Kilchling

1 Alle Links zu den elektronisch verfügbaren Ressourcen finden sich auf der Projektseite unter www.mpicc.de/ww/de/pub/ forschung/forschungsarbeit/kriminologie/tk_verbindungsdaten.htm

2 Richtlinie 2006/24/EG vom 15. März 2006 über die Vorratsspeicherung von Daten, die bei der Bereitstellung öffentlich zugänglicher elektronischer Kommunikationsdienste oder öffentlicher Kommunikationsnetze erzeugt oder verarbeitet werden, ABI. L 105, S. 54.

3 Bundesgesetz zur Neuregelung der Telekommunikationsüberwachung und anderer verdeckter Ermittlungsmaßnahmen vom 21. Dezember 2007, BGBI. I, S. 3198. 


\title{
I. Verbrechensfurcht
}

\author{
Schützt soziale Sicherheit vor Kriminalitätsfurcht?
}

Krimonologische

Forschungsabteilung

Forschungsschwerpunkt: Innere Sicherheit, organisierte Kriminalität, Terrorismus gesellschaftliche Wahrnehmung

Ein bedeutsamer Ansatz komparativer, international ausgerichteter kriminologischer Forschung ist die länderübergreifende Erhebung und Analyse von Dunkelfelddaten. Hierzu zählen insbesondere Opfer- und weiterführende Einstellungsdaten. Das hier präsentierte Forschungsprojekt befasst sich mit Auswertungen des European Crime and Safety Surveys, der eingebunden in die fünfte Welle des International Crime Victim Surveys 2004/2005 durchgeführt wurde. Es handelt sich hierbei um eine repräsentative Bevölkerungsbefragung in 18 europäischen Ländern, darunter auch die neuen EUMitgliedsländer Polen, Ungarn und Estland. Der inhaltliche Schwerpunkt der Umfrage liegt auf persönlichen Kriminalitätserfahrungen und -wahrnehmungen. Organisiert wurde diese Umfrage durch ein europäisches Konsortium bestehend aus Gallup Europe, UNICRI (Italien), Gallup Hungary, dem Max-Planck-Institut für ausländisches und internationales Strafrecht in Freiburg, CEPS/INSTEAD (Luxemburg) sowie GeoX Ltd. (Ungarn). Das Forschungsprojekt nutzt diese Befragungsdaten, um Länderunterschiede in Viktimisierungserfahrungen und kriminalitätsbezogenen Einstellungen theoretisch und empirisch näher zu erforschen. Dabei wird die Analyse individueller und kontextbezogener Einflussfaktoren in Mehrebenenanalysen verbunden. Die Datenanalysen basieren neben dem European Crime and Safety Survey 2005 (EU-ICS) auf weiteren repräsentativen Bevölkerungsbefragungen in Europa, v.a. auf dem European Social Survey (ESS), der bislang in drei Wellen durchgeführt wurde. Die Verwendung unabhängiger Datensätze ermöglicht zum einen die Berücksichtigung von wichtigen Kontrollvariablen, die nicht in allen Datensätzen enthalten sind, sowie eine Überprüfung der Robustheit und Replizierbarkeit der Ergebnisse.

Im Mittelpunkt des Interesses der aktuellen Auswertungen steht die Frage nach der Beziehung zwischen wohlfahrtsstaatlicher Politik und subjektivem Sicherheitsgefühl im europäischen Ländervergleich. Die bisherige Forschung hat das Ausmaß an Kriminalitätsfurcht in erster Linie mit individuellen Merkmalen, Lebensstilen, vergangenen Kriminalitätserfahrungen sowie mit situativen und lokalen Bedingungen in Verbindung gebracht. Die Untersuchung der Wirkung von gesellschaftlichen Einflüssen blieb bisher weitgehend unberücksichtigt, obgleich festgestellt werden kann, dass sich die europäischen Länder im Hinblick auf die Kriminalitätsfurcht ihrer Bürger erheblich voneinander unterscheiden (vgl. Abbildung 1).

Folgt man der Wohlfahrtsstaatentypologie EspingAndersens (1990, 1999), so zeigt sich ein ausgeprägter Zusammenhang zwischen verschiedenen sozialpolitischen Systemen - den sogenannten Wohlfahrtsregimen - und dem Kriminalitätsfurchtniveau: Ein hohes Maß an Kriminalitätsfurcht kann in erster Linie in den osteuropäischen und angelsächsischen Ländern beobachtet werden, während die Bürger der skandinavischen Länder vergleichsweise geringe Viktimisierungsängste aufweisen (vgl. Abbildung 1). Diese beobachtbaren nationalen Unterschiede in der Kriminalitätsfurcht (ebenso wie kleinräumliche und individuelle Unterschiede) können dabei nicht allein auf Unterschiede in der objektiven Kriminalitätslage zurückgeführt werden, denn Bedrohungsgefühle und faktische Kriminalität verlaufen nicht immer parallel. Die Wirkmechanismen, die dem Zusammenhang zwischen Wohlfahrtsregime und Kriminalitätsfurcht zugrunde liegen, sind bislang nur wenig untersucht worden. Das Forschungsprojekt möchte diese Forschungslücke schließen und geht daher der Frage nach, welche Einflüsse auf gesellschaftlicher Ebene Kriminalitätsfurcht moderieren und den Zusammenhang zwischen Wohlfahrtsregimetyp und Kriminalitätsfurcht begründen.

Den theoretischen Ausgangspunkt bildet die ,Generalisierungsthese', die Kriminalitätsfurcht nicht als spezifische Reaktion auf faktische Kriminalitätsrisiken sieht, sondern vielmehr als Ausdruck latenter und diffuser Existenzängste (vgl. Boers 1991; Hirtenlehner 2006; Jackson 2004). Die Angst, Opfer einer Straftat zu werden, ist aus 


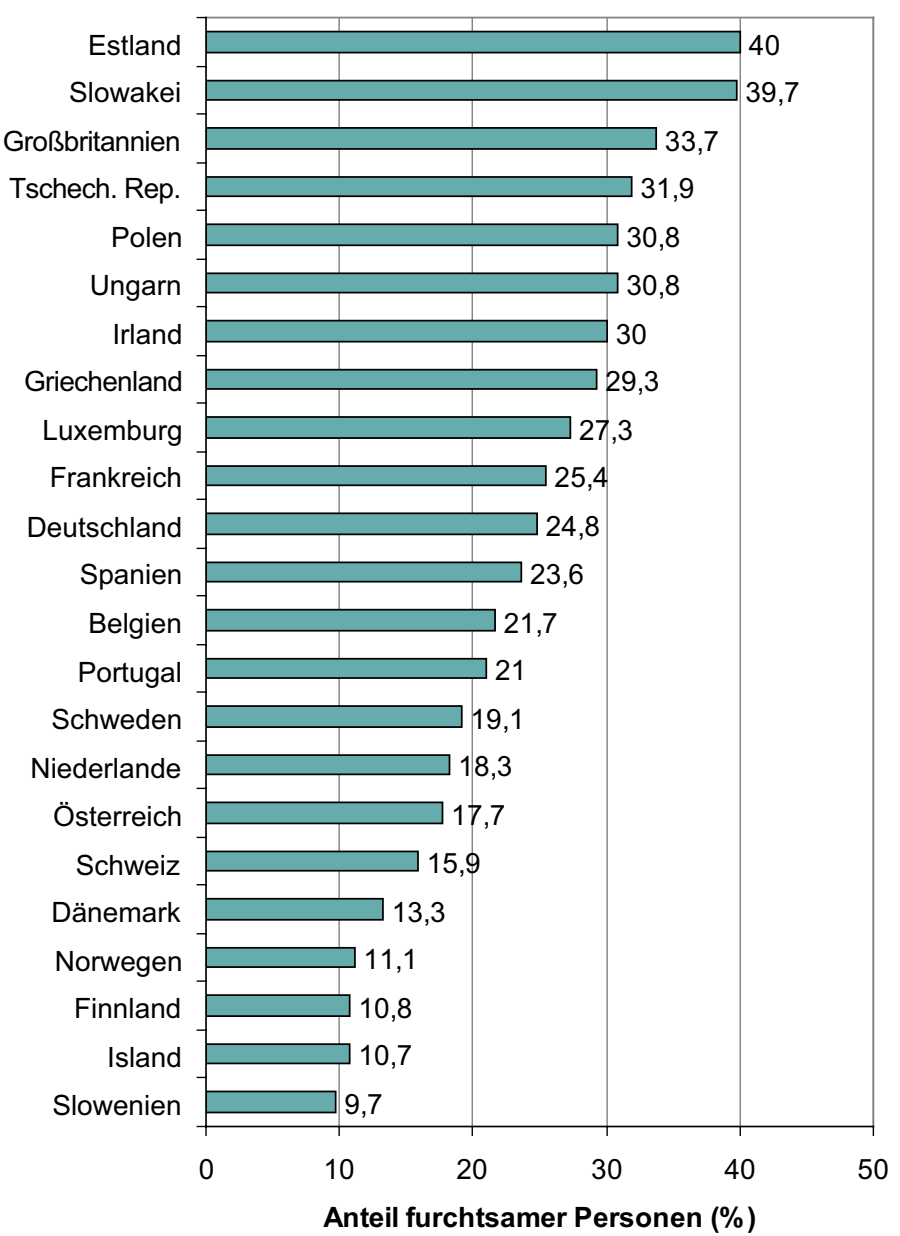

dieser Perspektive keine spezifische Angst vor Kriminalität, sondern Ausdruck einer allgemeinen gesellschaftlichen Unsicherheit, die sich lediglich (neben anderen Bereichen) der Kriminalität bedient. Ausgehend von diesen Überlegungen lässt sich vermuten, dass der Schutz vor sozialen Risiken (wie z.B. Armut und Einkommensverluste aufgrund von Alter, Krankheit, Arbeitslosigkeit und der Geburt von Kindern) eine bedeutende Rolle zur Erklärung von subjektiven Unsicherheitsgefühlen besitzt. Da der Wohlfahrtsstaat durch seine sozialpolitischen Interventionen und Programme in der Lage ist, derartige soziale Risiken abzufangen und dem Individuum eine existenzielle Grundsicherung zu garantieren, ist davon auszugehen, dass die Art und das Ausmaß sozialpolitischer Einflussnahme für das generelle Sicherheitsgefühl von Bedeutung sind und darüber auch Kriminalitätsängste reduziert werden können. Folgende Untersuchungsfragen leiten die empirischen Analysen: Inwiefern besteht eine Beziehung zwischen wohlfahrtsstaatlicher Politik und Kriminalitätsangst? Welche sozialpolitischen Maßnahmen können am effektivsten Viktimisierungsängste reduzieren?
Die individuellen Befragungsdaten der aktuellen Auswertungen stammen aus der zweiten Welle des European Social Survey (ESS 2004). Die Zielvariable in den Analysen bildet das sogenannte Kriminalitätsfurcht-Standarditem mit der Formulierung: „How safe do you feel walking alone in your area after dark?". Die möglichen Antwortkategorien lauten: „very safe“, „safe“, „unsafe“ oder „very unsafe“. Für die Analysen wurden die vier möglichen Antworten in zwei Ausprägungen „very safe / safe“ und „unsafe / very unsafe“ zusammengefasst. Individuelle und haushaltskontextuelle Einflussfaktoren, deren Wirkung auf das subjektive Sicherheitsgefühl in den Analysen Berücksichtigung findet, sind Alter, Bildung, Geschlecht, Erwerbsbeteiligung, Wohnortsgröße, persönliche und kommunizierte Kriminalitätserfahrungen, Fernsehkonsum, Haushaltszusammensetzung, Haushaltseinkommen sowie die Kontakthäufigkeit mit Freunden, Verwandten und Nachbarn.

Zur Überprüfung des Einflusses von Sozialschutzleistungen, die den theoretischen Überlegungen zufolge mit dem Ausmaß an Kriminalitätsfurcht in Verbindung stehen, werden neben den Indivi-
Abbildung 1:

Anteil der Personen mit Kriminalitätsfurcht im europäischen Ländervergleich

Anmerkung: dichotomisiertes Standarditem „How safe do you feel walking alone in your area after dark?"

Datenquelle: ESS 2004; gewichtete Daten 


\section{Abbildung 2:}

Zusammenhang zwischen Kriminalitätsfurcht und öffentlichen Bildungsausgaben Datenquellen: ESS 2004; Eurostat

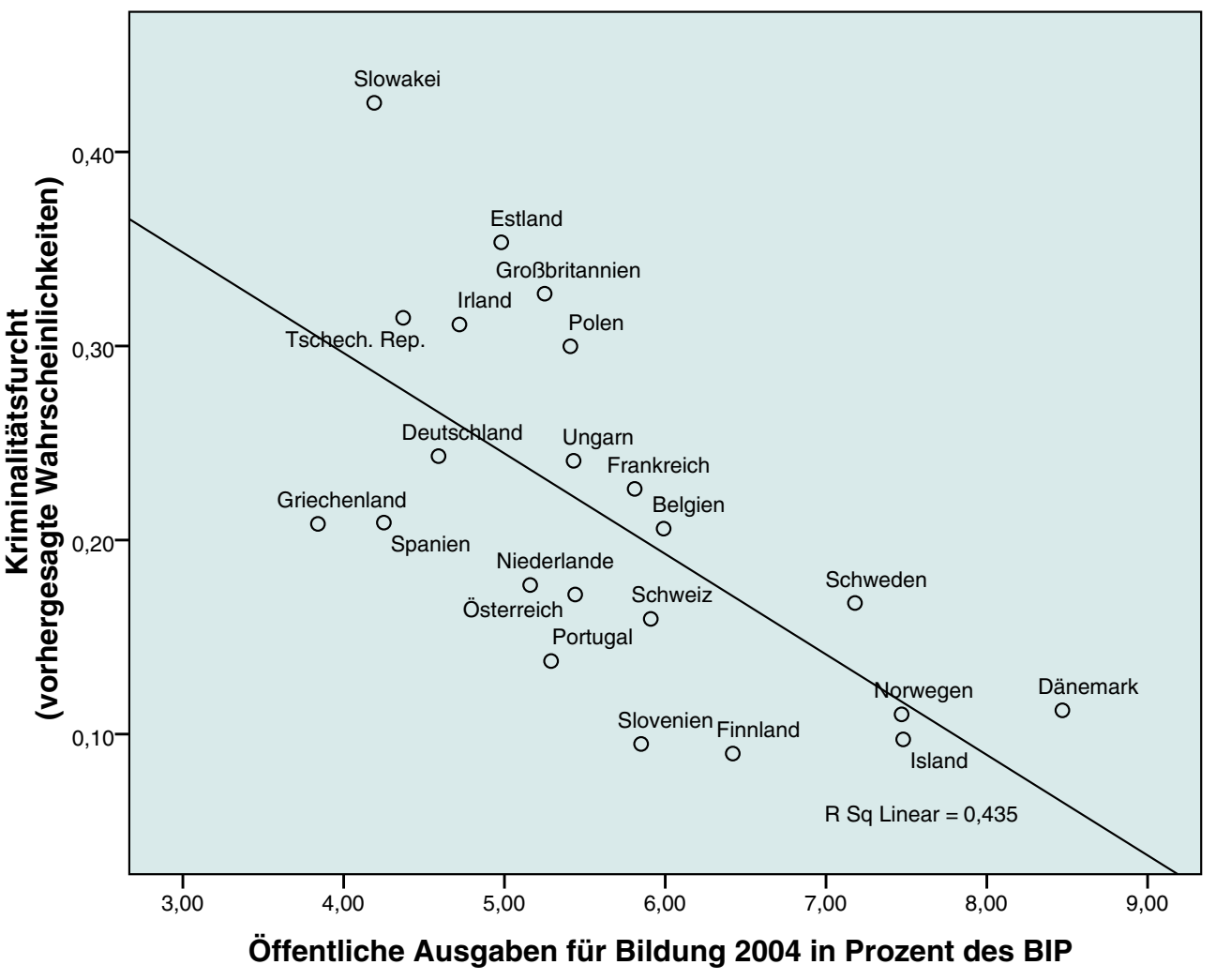

dualdaten verschiedene Arten von Wohlfahrtsindikatoren in die Analysen einbezogen. Zu diesen zählen beispielsweise die Sozialausgaben (in \% des BIP), öffentliche Bildungsausgaben (in \% des BIP), ein Dekommodifizierungs-Index (der die Abhängigkeit des Individuums vom Markteinkommen abbildet), die öffentlichen Ausgaben für Gesundheit, Invalidität und Gebrechen, Alter, Arbeitslosigkeit, Wohnverhältnisse und soziale Ausgrenzung (jeweils in \% des BIP), aber auch Indikatoren, die kulturelle Unterschiede zwischen den Ländern (z.B. Einstellungen zur Familie) berücksichtigen. Die meisten dieser Kontextdaten konnten beim Statistischen Amt der Europäischen Gemeinschaften (EUROSTAT) oder bei der Organisation für wirtschaftliche Zusammenarbeit und Entwicklung (OECD) bezogen werden.

Das empirische Analysemodell kombiniert damit zwei Ebenen von Daten: zum einen die nationalen Kontextdaten und zum anderen individuelle Befragungsdaten. Zur statistischen Analyse einer derartigen hierarchischen Datenstruktur (Individuen in übergeordneten Länderkontexten) wurde die Mehrebenenanalyse entwickelt, die den komplexen Zusammenhängen zwischen den verschiedenen Ebenen Rechnung trägt (vgl. Raudenbush/ Bryk 2002; Snijders/Bosker 1999). Mehrebenen- analysen erlauben das simultane Schätzen von Effekten der Individual- und Kontextebene. Konkret lässt sich dadurch die individuelle Kriminalitätsfurcht nicht nur auf individuelle Merkmale wie Alter, Geschlecht, Bildung, Fernsehkonsum etc. zurückführen, sondern es kann gleichzeitig die Wirkung von spezifischen sozialpolitischen und anderen gesellschaftlichen Bedingungen auf die individuelle Angst vor Kriminalität erfasst werden. Darüber hinaus ermöglicht die Mehrebenenanalyse auch die Untersuchung von Wechselwirkungen zwischen individuell-persönlichen und kontextuellen Faktoren.

Erste empirische Befunde bestätigen den vermuteten Zusammenhang zwischen wohlfahrtsstaatlichen Leistungen und Kriminalitätsfurcht: Mit dem Ausmaß an Sozialschutzleistungen nimmt Kriminalitätsangst deutlich ab. Demnach scheinen sozialpolitische Maßnahmen erwartungsgemäß einen negativen Einfluss auf das Kriminalitätsfurchtniveau zu besitzen. Zugleich zeigen die Analysen, dass nicht alle wohlfahrtsstaatlichen Leistungen gleichermaßen Kriminalitätsfurcht senken, denn einige sozialpolitische Instrumente befördern das subjektive Sicherheitsgefühl stärker als andere. Als ausgesprochen effektiv diesbezüglich erweisen sich die staatlichen Sachleistungen 
für Familien (die in erster Linie öffentliche Aufwendungen für Kinderbetreuungseinrichtungen, aber auch Unterbringung in Einrichtungen und Pflegefamilien sowie häusliche Hilfe umfassen) sowie die öffentlichen Bildungsausgaben (vgl. Abbildung 2).

Die Ursache für diese ausnehmend furchtreduzierende Wirkung der beiden sozialpolitischen Interventionen liegt möglicherweise in einem besonders aktivierenden und präventiven Effekt. Es scheint, als würden gerade diejenigen wohlfahrtsstaatlichen Leistungen ein größeres Sicherheitsgefühl produzieren, die die individuelle Handlungsfähigkeit und damit auch die individuelle Kapazität, mit sozialen Risiken umzugehen, stärken. Bei den Bildungsausgaben und Familiensachleistungen handelt es sich um nicht-monetäre staatliche
Transfers. Das bedeutet, sie kompensieren nicht wie Barleistungen (z.B. Arbeitslosengeld, Erziehungsgeld, Renten) unmittelbar Einkommen und Einkommensdefizite, sondern tragen vielmehr zur Selbsthilfe und Eigeninitiative bei, indem sie die Arbeitsmarktpartizipation und aktive Teilhabe am gesellschaftlichen Leben anregen. Die Bildungsinstitutionen und die Kleinkindbetreuung sind wichtige Kanäle, die über Gleichheit und Ungleichheit von Lebenschancen und Lebensrisiken innerhalb einer Gesellschaft entscheiden und darüber vermutlich auch Kriminalitätsfurcht mindern können (vgl. Allmendinger 1999). Die genauen Zusammenhänge werden derzeit noch näher untersucht.

Dina Hummelsheim, Dietrich Oberwittler

Literaturhinweise: Allmendinger, Jutta (1999). Bildungsarmut - zur Verschränkung von Bildungs- und Sozialpolitik. Soziale Welt 50(1), 35-50. Boers, Klaus (1991). Kriminalitätsfurcht. Pfaffenweiler: Centaurus-Verlagsgesellschaft. Esping-Andersen, Gøsta (1990). The three worlds of welfare capitalism. Cambridge: Polity Press. Esping-Andersen, Gøsta (1999). Social Foundations of Postindustrial Economies. Oxford: University Press. Hirtenlehner, Helmut (2006). Kriminalitätsfurcht - Ausdruck generalisierter Ängste und schwindender Gewissheiten? Untersuchung zur empirischen Bewährung der Generalisierungsthese in einer österreichischen Kommune. Kölner Zeitschrift für Soziologie und Sozialpsychologie 58(2), 307-331. Jackson, Jonathan (2004). Experience and Expression, Social and Cultural Significance in the Fear of Crime. British Journal of Criminology 44: $946-$ 966. Raudenbush, Stephen W./ Bryk, Anthony S. (2002). Hierarchical Linear Models. Applications and Data Analysis Methods. Thousand Oaks, London, New Delhi: Sage Publications. Snijders, Tom A.B./ Bosker, Roel J. (1999). Multilevel Analysis. London, Thousand Oaks, New Delhi: Sage Publications. 


\section{J. Strafrecht und Kriminologie unter einem Dach}

Grenzen des Rechtsgüterschutzes vor dem Bundesverfassungsgericht

Eine Sonderbeilage der Wochenzeitschrift „Die Zeit“ und zahlreiche weitere Reportagen in Zeitungen, Rundfunk und Fernsehen berichteten 2007 und 2008 ausführlich über einen tragischen Fall von Geschwisterliebe: Patrick S. und seine sieben Jahre jüngere, geistig leicht behinderte Schwester Susan K. wuchsen seit frühester Jugend getrennt auf: Er kam im Alter von drei Jahren in ein Heim und dann zu einer Adoptivfamilie. Sie wurde erst danach geboren und blieb auch nach der Scheidung der Eltern bei der Mutter. Beide lernten sich im Jahr 2000 kennen, als Susan 16 und Patrick 23 Jahre alt waren. In der Folge zog Patrick zu Mutter und Schwester. Nachdem die Mutter kurz darauf verstarb, verliebten sich die weiterhin zusammenlebenden Geschwister und bekamen vier Kinder, von denen zwei leicht behindert sind. Die drei ältesten Kinder wurden dem Paar vom Jugendamt weggenommen. Patrick wurde wegen Beischlafs zwischen Verwandten mehrfach nach $\$ 173$ Strafgesetzbuch verurteilt, zuletzt zu einer Freiheitsstrafe von insgesamt zweieinhalb Jahren - seine freiwillige Sterilisation half ihm nichts. Nach Rechtskraft des Urteils griff sein Rechtsanwalt die Verurteilung wegen Beischlafs zwischen Geschwistern beim Bundesverfassungsgericht an und beantragte die Feststellung der Verfassungswidrigkeit der Strafbestimmung über Geschwisterinzest (\$173 Abs. 2 Satz 2 StGB).

Dieser Fall war nicht nur wegen seiner menschlichen Schicksale und zahlreicher anderer Umstände spektakulär. Er wirft vor allem auch brisante Rechtsfragen auf: Welche Rechtsgüter schützt der Inzesttatbestand? Religiöse Werte? Moral? Tabus? Familie? Volksgesundheit und/oder Genetik? Ist der Schutz dieser Werte in einem säkularen Strafrecht legitim und kann das Strafrecht diese Aufgaben überhaupt erfüllen? Wie sind entsprechende Fälle im ausländischen Strafrecht geregelt? Wie häufig sind Inzesthandlungen zwischen Geschwistern und ist ein entsprechender Straftatbestand überhaupt erforderlich, wenn das Inzest-Tabu in anderen Staaten auch ohne Strafrechtsschutz gilt? All diese Fragen sind deswegen so brisant, weil sie am Beispiel des Inzests die grundlegenden Fragen nach den Grenzen eines rational begründbaren Strafrechts aufwerfen.

Das Max-Planck-Institut für ausländisches und internationales Strafrecht verfügt über die Kompetenz zur Beantwortung derartiger Fragen vor allem aufgrund seiner beiden Schwerpunkte auf den Gebieten der Strafrechtsvergleichung und der Kriminologie, deren Verbindung der Institutsgründer Prof. Dr. Hans-Heinrich Jescheck mit der Zielsetzung von „Strafrecht und Kriminologie unter einem Dach“ umschrieben hatte. Das Bundesverfassungsgericht beauftragte die beiden Institutsdirektoren deswegen mit einem gemeinsamen Sachverständigengutachten. Dabei ging es nicht um die Beurteilung der - allein dem Verfassungsgericht obliegenden - Rechtsfrage nach der Nichtigkeit der Inzeststrafbestimmung. Aufgabe des Gutachtens war vielmehr die Beantwortung eines ausführlichen Fragenkatalogs zu den rechtsvergleichenden, kriminologischen, eugenischen und medizinischen Grundlagen dieser Rechtsfrage.

Der folgende Beitrag stellt zunächst die rechtsvergleichenden und dann die kriminologischen Ergebnisse dar, die das Institut dem Gericht übermittelte. Er geht dann noch kurz auf die in der Sache ergangene Entscheidung des Bundesverfassungsgerichts ein.

\section{Inzeststrafbarkeit im internationalen Rechtsvergleich}

Der strafrechtliche Teil des vom Bundesverfassungsgericht in Auftrag gegebenen Gutachtens sollte vor allem klären, in welchen Rechtsordnungen einvernehmliche sexuelle Handlungen zwischen Familienangehörigen unter Strafe stehen und wie die Entscheidung für oder gegen eine Pönalisierung begründet wird. Weiter stellte sich die Frage, ob und inwieweit unabhängig von 
der Inzestnorm andere Bestimmungen des Strafrechts einen ausreichenden Schutz vor denjenigen inzestuösen Handlungen gewähren, die unter erschwerten Bedingungen, insbesondere unter Anwendung von Gewalt, an Kindern oder im Rahmen von Autoritäts- oder Abhängigkeitsverhältnissen begangen werden.

Diese rechtsvergleichende Fragestellung wurde mit der Methode der funktionalen Strafrechtsvergleichung angegangen, bei der die Rechtsordnungen von zunächst 20 und dann später 22 Ländern aus repräsentativ ausgesuchten Rechtssystemen und Regionen untersucht wurden. Für eine erfolgreiche Bewältigung der rechtsvergleichenden Aufgabe war dabei die richtige Bestimmung des Vergleichsgegenstands im Sinne der funktionalen Rechtsvergleichung von entscheidender Bedeutung: Vergleichsgegenstand im Sinne der funktionalen Rechtsvergleichung konnte und durfte nicht der Inhalt des Rechtsbegriffs „Inzest" sein, sondern wegen des unterschiedlichen Verständnisses dieses Rechtsbegriffs in den verschiedenen Rechtsordnungen nur ein entsprechender Lebenssachverhalt. Dieser wurde im vorliegenden Fall auf die Vornahme von einverständlichen sexuellen Handlungen zwischen erwachsenen Familienangehörigen beschränkt, da nur für diesen Lebenssachverhalt die Frage zu beantworten ist, ob und welche Schutzaspekte eine solche Strafbestimmung rechtfertigen können. Rechtliche Regelungen, die sexuelle Handlungen zwischen Familienangehörigen nur unter qualifizierten Bedingungen unter Strafe stellen (insbesondere bei Einsatz von Gewalt, bei Minderjährigkeit eines Beteiligten oder bei Missbrauch eines Autoritäts- oder Abhängigkeitsverhältnisses), wurden nicht in den Vergleich der „Inzestbestimmungen im engeren Sinne“ einbezogen, sondern gesondert verglichen, auch unter dem Gesichtspunkt, ob sich ohne eine Inzestbestrafung in diesen Fällen gegebenenfalls Schutzlücken ergeben können.

\section{Strafbarkeit des Inzests}

Die Ergebnisse des Gutachtens zeigen, dass zahlreiche Rechtsordnungen ohne eine Inkriminierung des einverständlichen Inzests zwischen Erwachsenen auskommen. In etwa einem Drittel der untersuchten Rechtsordnungen besteht eine solche Strafbarkeit nicht: Vor allem in Frankreich nach dem Code Napoléon von 1811 sowie in den durch das französische Recht beeinflussten Rechtsordnungen (in den Niederlanden, der Türkei und im modernen Recht von Côte d'Ivoire), fer- ner in Russland und China, schließlich auch in Spanien, Israel sowie innerhalb der USA in den Staaten Rhode Island, New Jersey und Michigan. Einen Straftatbestand des Inzests gibt es hingegen in 14 der 22 einbezogenen Länder: in allen australischen Staaten und Territorien, Chile, Dänemark, Deutschland, England (und Wales), Griechenland, Italien, Kanada, Polen, Rumänien, Schweden, der Schweiz, Ungarn sowie in nahezu allen Staaten der USA.

\section{Begründung der Inzeststrafbarkeit bzw. der Nichtbestrafung}

Die Begründung für die Strafbarkeit des Inzests ist in den verschiedenen Rechtsordnungen sehr uneinheitlich. In der Praxis herrschen Mischmodelle vor, die verschiedene Strafbegründungen miteinander kombinieren. Religiöse Begründungen spielen heute außerhalb des islamischen Strafrechts kaum noch eine Rolle. Auch moralische Erwägungen treten zunehmend in den Hintergrund und werden meist nur implizit herangezogen, da Aufgabe des Strafrechts in vielen aufgeklärten Staaten nicht die Aufrechterhaltung der Moral ist, sondern der Schutz von Rechtsgütern gegen sozialschädliches Verhalten. Bisweilen wird auch die Aufrechterhaltung des Inzesttabus in der Gesellschaft als mögliche Strafbegründung herangezogen; hier gelten jedoch ähnliche Bedenken wie gegen den strafrechtlichen Schutz der Moral.

Eugenische Gesichtspunkte der Verhinderung von „erbkrankem Nachwuchs“ werden heute ebenfalls nicht mehr alleine herangezogen; in einigen Rechtsordnungen werden sie überhaupt nicht als relevant angesehen, andere Rechtsordnungen wiederum rekurrieren zumindest ergänzend auf genetische Gefahren. Diese Begründungen gelten jedoch insbesondere deswegen als problematisch, weil die genetischen Risiken des Inzests teilweise nicht höher als andere genetische Risiken eingestuft werden, weil eine Beurteilung der Zeugung behinderter Kinder als Schaden das Lebensrecht dieser Kinder negiert, weil die mit dem Risiko von missgebildeten Kindern verbundene Zeugung in anderen Fallgestaltungen nicht bestraft wird und weil gegen dieses Risiko erfolgreicher mit Aufklärung und Verhütung als mit einem Strafverbot des Geschlechtsverkehrs vorgegangen werden kann.

Demgegenüber ist der Aspekt des Familienschutzes in vielen Rechtsordnungen von hoher Bedeutung. Eine solche Begründung ist jedoch unter ande- 
rem deswegen fragwürdig, weil die Schädigung der Familie meist nicht die Folge, sondern die Ursache für Inzest ist; teilweise wird auch darauf hingewiesen, dass das klassische Leitbild der Familie heute erodiert ist. Eine inhaltliche Analyse der Strafnormen führt darüber hinaus in vielen Rechtsordnungen auch zu widersprüchlichen Ergebnissen, da die Strafbegründungen meist nicht konsequent in die entsprechenden Tatbestandsfassungen umgesetzt sind. So müsste z.B. eine eugenische Strafbegründung homosexuellen Verkehr straflos lassen, während ein Schutz der Familie konsequenterweise auch Adoptivkinder und -geschwister einbeziehen sollte.

Die Nichtbestrafung inzestuösen Verhaltens wird meist damit begründet, dass das Strafrecht nicht die Aufgabe habe, freiwillig begangene sexuelle Handlungen unter Erwachsenen zu bestrafen. Für den Schutz vor Gewalt oder Missbrauch sowie den Schutz von Minderjährigen seien die allgemeinen Sexualdelikte bzw. deren Sondertatbestände ausreichend. Ansonsten genüge das gesellschaftliche Tabu. In Russland und China beruhte die Abschaffung der Inzeststrafbarkeit hingegen im Wesentlichen auf ideologischen Gründen.

\section{Reichweite der Inzeststrafbarkeit}

Besteht eine Inzestnorm, so ist bei ihrer Ausgestaltung ein Kernbereich von Rechtsordnungen feststellbar, die eine Strafbarkeit des Inzests auf fortpflanzungserhebliche Handlungen zwischen nahen Blutsverwandten beschränken.

In den meisten (d.h. in neun) Rechtsordnungen kommen als taugliche Täter der Inzesttat daher nur Verwandte in gerader Linie sowie Voll- und Halbgeschwister in Betracht. In nur wenigen (drei) Staaten wird der Anwendungsbereich auf weitere Blutsverwandte erstreckt. Nicht viel häufiger ist die Einbeziehung von Adoptivverwandtschaften. Nur ganz vereinzelt fallen Stiefverhältnisse und Verschwägerte unter den Inzesttatbestand.

Als Tathandlung kennen einige (sechs) Rechtsordnungen nur den heterosexuellen Vaginalverkehr, die übrigen Inzestbestimmungen erfassen auch (homo- oder heterosexuellen) Oral- oder Analverkehr. Nur in wenigen (vier) Rechtsordnungen sind darüber hinaus inzestuöse sexuelle Handlungen strafbar, die keine Penetration beinhalten. Nicht rein sexuelle Beziehungen wie Heirat oder eheähnliches Zusammenleben werden lediglich in den USA einbezogen.
Bei den Sanktionen ergibt sich ein sehr differenziertes Bild. Die verschiedenen untersuchten Inzesttatbestände enthalten teils einen einheitlichen, teils einen nach der Art des Verwandtschaftsverhältnisses, nach der Tathandlung oder nach anderen Gesichtspunkten differenzierten Strafrahmen. Die Strafhöhe reicht von einer Höchststrafe von sechs Monaten bis zu lebenslanger Freiheitsstrafe. In den meisten Rechtsordnungen ist die Tat jedoch mit maximal zwei bis fünf Jahren Freiheitssstrafe bedroht.

\section{Erfassung inzestuöser Handlungen durch Delikte mit anderen Schutzzwecken}

Da der mögliche Unwertgehalt der meisten bekannt werdenden Inzestfälle faktisch nicht nur in einvernehmlichen sexuellen Handlungen zwischen Familienangehörigen besteht, sondern in zusätzlichen und spezifischen Begleitumständen der Tat, wurden auch Tatbestände untersucht, die eine andere Schutzrichtung haben als die reinen Inzestnormen. Dabei geht es vor allem um den Einsatz von Gewalt, das jugendliche Alter eines Beteiligten sowie das Ausnutzen eines besonderen Autoritäts-, Abhängigkeits- oder Vertrauensverhältnisses.

Vereinzelt sehen sowohl Rechtsordnungen mit als auch ohne Inzeststrafbarkeit einen Erschwerungsgrund vor, wenn ein allgemeines Sexualdelikt durch bestimmte Familienangehörige begangen wird. Ebenfalls unabhängig von der Inkriminierung des freiwilligen Inzests zwischen Erwachsenen sind die Regelungen ausgestaltet, die bei sexuellen Handlungen an Kindern oder im Rahmen von bestimmten Autoritäts- oder Abhängigkeitsverhältnissen eingreifen. Bei diesen Delikten ist der erfasste Personenkreis nie auf Blutsverwandte beschränkt, sondern es ist ein weiterer Kreis von Familienangehörigen einbezogen. Hieraus lässt sich erkennen, dass der Schutzzweck dieser Normen ein anderer ist. In den meisten Fällen ist innerhalb solcher Abhängigkeitsverhältnisse die Altersgrenze auf 18 Jahre heraufgesetzt.

\section{Außerstrafrechtliche Aspekte}

In allen an der Untersuchung beteiligten Ländern ist einverständlicher Inzest gesellschaftlich stark tabuisiert. Dabei gehen die gesellschaftlichen Tabus weit über die gegebenenfalls bestehenden Straftatbestände hinaus. Sie sind nicht auf 
Blutsverwandte beschränkt, sondern erfassen das gesamte familiäre Umfeld. Aus diesen Gründen sind Inzestpaare ebenso wie die eventuell aus dieser Verbindung hervorgegangenen Kinder häufig starken Ausgrenzungen ausgesetzt. Daraus lässt sich aber nicht automatisch schließen, dass der einverständliche Inzest zwischen Erwachsenen nach der gesellschaftlichen Meinung auch tatsächlich mit den Mitteln des Strafrechts sanktioniert werden muss. In allen untersuchten Rechtsordnungen ist ferner die Eheschließung zwischen bestimmten Verwandten ausgeschlossen. Die persönliche Reichweite dieser Eheverbote ist allerdings sehr unterschiedlich ausgestaltet. In den meisten Gesellschaften dürfte das Inzesttabu damit auch unabhängig von entsprechenden Strafnormen gelten und - wie die Situation in Frankreich seit der Aufklärung und dem Code Napoléon zeigt - auch ohne dieses bestehen bleiben.
Fazit

Die rechtsvergleichende Analyse zeigt damit, dass eine Gesellschaft auch ohne Inzeststrafbestimmung auskommen kann, die den einverständlichen Sexualverkehr zwischen Erwachsenen kriminalisiert. Entscheidend sind dagegen Strafnormen im Hinblick auf den Einsatz von Gewalt, das jugendliche Alter eines Beteiligten sowie das Ausnutzen eines besonderen Autoritäts-, Abhängigkeits- oder Vertrauensverhältnisses. Der Rechtsvergleich macht darüber hinaus die Schwierigkeiten deutlich, eine rationale Begründung für eine entsprechende Strafbestimmung zu finden. $\mathrm{Ob}$ ein Inzeststraftatbestand erforderlich ist und ob die geltend gemachten Rechtsgüter tatsächlich anzuerkennen sind, muss deswegen zunächst durch eine kriminologische und genetische Analyse näher untersucht werden.

\section{Inzest in der Kriminologie und der Genetik}

Die kriminologischen Fragestellungen des vom Bundesverfassungsgericht erbetenen Gutachtens bezogen sich auf die Verbreitung des (einverständlichen) Geschwisterinzests, die Folgen des Inzests in Form der Auswirkungen auf Personen und Familie, und die sogenannte ,natürliche Inzestscheu“, genetische Risiken und schließlich auf die Entwicklung der polizeilich registrierten Inzestfälle sowie ihre justizielle Behandlung. Der Zugang zu diesen Fragen basierte auf Sekundäranalysen sexualwissenschaftlicher, kriminologischer, soziologischer, psychologischer und medizinischer Forschung sowie Analysen von Polizei- und Strafverfolgungsstatistiken, ferner auf Auswertungen der Daten der Freiburger Kohortenuntersuchung.

\section{Inzidenz, Prävalenz und das Dunkelfeld des Geschwisterinzests}

Empirisches Wissen zur Häufigkeit des Inzests hat Bedeutung für die Antwort auf die Frage, ob das strafrechtliche Verbot einen relevanten Beitrag für den Rechtsgüterschutz leistet. Rechtsordnungen mit und ohne strafrechtliches Inzestverbot könnten insoweit eine quasi-experimentelle Grundlage bieten.

Die Sekundäranalyse empirischer Forschung zu Inzidenz (Häufigkeit von inzestuösen Handlungen, Dauer und Intensität von inzestuösen Beziehungen) und Prävalenz des Geschwisterinzests
(Anteil einer bestimmten Population, der jedenfalls einmal inzestuöse Handlungen begangen hat oder inzestuösen Handlungen ausgesetzt war) verweist aber auf eine nur beschränkte Datenlage, die keine sicheren Rückschlüsse auf die Frage erlaubt, mit welcher Häufigkeit inzestuöse Handlungen zwischen Geschwistern auftreten und wie sich die Dauer sowie die Intensität der Beziehungen entwickeln. Die ältere wie auch die neuere Forschung zur menschlichen Sexualität unterscheiden nicht danach, ob sexuelle Handlungen innerhalb oder außerhalb der Familie auftreten. Sexualwissenschaftliche Forschungen sind zunächst an allgemeinen Verteilungen sexueller Präferenzen und Einstellungen interessiert; sie verfolgen in den letzten Jahrzehnten vor allem gesundheitspolitische Fragestellungen und Probleme der Behandlung sexueller Abweichungen (darunter insbesondere die Pädophilie).

Die Fragestellung inzestuöser Handlungen in der Familie (und im sozialen Nahraum) wird allerdings ab Mitte der 1970er Jahre mit Untersuchungen zum sexuellen Missbrauch aufgegriffen. In dieser Forschungslinie wird der Inzest weitgehend mit sexuellem Missbrauch gleichgesetzt. Insoweit ergeben sich Beschränkungen, die sich auf das Alter (das Forschungsinteresse konzentriert sich auf sexuelle Handlungen im Kindheits- und Jugendalter) sowie die Opferstellung (des kindlichen oder jugendlichen Sexualpartners) beziehen. Einvernehmliche sexuelle Handlungen zwischen 
erwachsenen (oder gleichaltrigen) Familienmitgliedern werden lediglich am Rande, miterfasst oder von vornherein ausgeschlossen. Befragungen richten sich im Schwerpunkt auf im Kindheitsund Jugendalter erfahrene sexuelle Übergriffe durch ältere (erwachsene) Familienangehörige. Konventionelle Dunkelfeldfbefragungen der Kriminologie (Selbstberichtsuntersuchungen und Opferbefragungen) erfassen sexuelle Handlungen lediglich am Rande und dann solche, die durch Gewalt oder andere Nötigungsmittel gekennzeichnet sind. Insoweit sind auf repräsentative empirische Untersuchungen gestützte Aussagen über die Prävalenz oder die Inzidenz weder für Deutschland noch für andere Länder möglich.

Aus vereinzelten Untersuchungen (überwiegend aus Nordamerika), die allerdings fast allesamt unter der Fragestellung des sexuellen Missbrauchs durchgeführt worden sind, und die von der Stichprobenziehung her gesehen nicht verallgemeinert werden können, lassen sich auch Hinweise auf nicht durch Gewalt und Zwang geprägte sexuelle Kontakte zwischen Geschwistern entnehmen. Auf der Grundlage dieser Untersuchungen lässt sich die Prävalenz des Geschwisterinzests (einvernehmlicher sexueller Verkehr) mit etwa 2 bis $5 \%$ angeben. Dabei handelt es sich ganz überwiegend um einmalige oder gelegentliche sexuelle Kontakte zwischen Geschwistern.

Insoweit lässt die Forschungslage eine Kontrastierung (und damit die Einschätzung der Relevanz der Strafandrohung für die Häufigkeit des Inzests) solcher Länder, in denen der Inzest nicht strafbar ist (z.B. Frankreich, Spanien oder südamerikanische Länder), mit solchen, in denen der Inzest unter Strafandrohung steht, auf der Grundlage von Prävalenz- und Inzidenzdaten nicht zu.

\section{Folgen des Inzests für die persönliche Entwicklung}

Für die Einschätzung des Unrechtsgehalts des Bruder-Schwester-Inzests sowie des Bedarfs an strafrechtlichem Schutz spielt die entwicklungspsychologische Schädlichkeit von Bruder-Schwester-Inzest eine Rolle. Hierzu wird eine anhaltende und kontroverse Debatte geführt. Freilich befassen sich - entsprechend den Defiziten in der Forschung zu Prävalenz und Inzidenz von Geschwisterinzest - nur wenige Studien auf der Grundlage quantitativer Daten mit den entwicklungspsychologischen Folgen einer solchen Verbindung. Dabei wird zwischen vorpubertären sexuellen Handlungen und Ereignissen nach Einsetzen der Pubertät unterschieden. Sexuelle Interaktionen zwischen Geschwistern vor der Pubertät können einvernehmlich eingeleitet werden und im Kontext normaler, spielerischer sexueller Entdeckungen liegen. Diese Annahme führt auch dazu, dass im Gegensatz zum Vater-TochterInzest Geschwisterinzest häufig nicht als Straftat oder abweichendes sexuelles Verhalten, sondern als normales, experimentelles Verhalten unter Kindern verstanden wird. Anhaltspunkte liegen dafür vor, dass inzestuöse Erfahrungen zwischen Geschwistern vor der Pubertät, auch wenn sie zunächst einvernehmlich erfolgen, in ein einseitig dominiertes Missbrauchsverhältnis umschlagen können. Hieraus sollen sich nachhaltige negative Auswirkungen auf die sexuelle Identitätsentwicklung und das Selbstbild der Beteiligten ergeben. Folgen aus einer inzestuösen Beziehung zwischen Geschwistern eine psychische Traumatisierung der Beteiligten und Risiken für die psychosexuelle Entwicklung und die Selbstwertentwicklung, so hängt die Stärke solcher Schädigungen jedenfalls vom Ausmaß des angewendeten Zwangs und der Dauer der inzestuösen Beziehung ab.

Ob es jedoch auch zu psychischer Traumatisierung kommt, wenn sich die beiden Beteiligten freiwillig zu sexuellen Kontakten auf der Basis gegenseitig erwiderter Liebe entscheiden, ist ungeklärt. Die Erkenntnisse zu den Folgen von Geschwisterinzest erlauben es derzeit nicht, spezifische entwicklungspsychologische Hypothesen zu überprüfen. Gerade in der Konstellation des Geschwisterinzests könnte es aber unter entwicklungspsychologischen Gesichtspunkten von großer Bedeutung sein, wie die entsprechenden Entwicklungsaufgaben in der Adoleszenz bewältigt werden. Zu diesen Entwicklungsaufgaben gehören der Umgang mit Sexualität und der Umbau der sozialen Beziehungen. Insoweit wird es auf die Ressourcen ankommen, die notwendig sind, um diese Entwicklungsaufgaben jeweils altersangemessen zu bewältigen. Dabei dürfte entscheidend sein, ob entsprechende Ressourcen vorhanden sind oder ob inzestuöse Beziehungen gerade deshalb eingegangen werden, weil die Ressourcen eben fehlen. Für Defizite in Ressourcen spricht, dass in Inzestfamilien das Fehlen einer protektiven Atmosphäre beobachtet wird.

Im Übrigen wird von einem Zusammenhang zwischen emotional vernachlässigenden Erziehungsmethoden und Geschwisterinzest berichtet. Dies lässt darauf schließen, dass Geschwister jedenfalls in länger andauernden sexuellen Beziehungen 
Defizite zu kompensieren suchen. Da freilich die Untersuchungen auf der Basis selektiver klinischer Gruppen durchgeführt worden sind, lässt sich die Inzidenz der entwicklungspsychologischen Folgeschäden von Geschwisterinzest bei den Beteiligten nicht bestimmen. Die Frage, ob Inzest zwischen erwachsenen Geschwistern unter entwicklungspsychologischen Gesichtspunkten zu Problemen führt, würde sich im Übrigen erschöpfend nur dann beantworten, wenn Inzestpaare und „Nicht“-Inzestpaare verglichen werden könnten. Komparative (Längsschnitt-)Analysen dieser Art gibt es bislang nicht.

\section{Inzest und die Folgen für die Familie}

Die Frage, ob der Geschwisterinzest als entwicklungspsychologisch schädlich für andere Familienmitglieder anzusehen ist, erstreckt sich auf eine soziale, gesellschaftliche Dimension. Dritte sehen sich mit einem gesellschaftlich tabuisierten Phänomen konfrontiert. Insofern können indirekte Schäden, so z.B. Ausgrenzung und/oder soziale Isolation aufgrund des Inzests in der Familie, entstehen. Von Inzest betroffene Familien sind allerdings bereits vor Auftreten des Geschwisterinzests durch eine Vielzahl sozialer und psychologischer Probleme belastet. Die Ergebnisse klinischer Studien adoleszenter Inzest-Täter zeigen, dass Gewalt und Dysfunktion in der Familie signifikante ätiologische Variablen für das Auftreten von Inzest sind. Geschwisterinzest wird daher übereinstimmend als eine Folge und nicht als die Ursache einer zerrütteten Familienstruktur angesehen. Diese Annahme wird auch durch die wenigen und im Wesentlichen in der ersten Hälfte des 20. Jahrhunderts liegenden empirischen Untersuchungen zur Kriminologie des Inzests gestützt. Die Untersuchungen verweisen darauf, dass sich polizeilich registrierte Inzestfälle ausschließlich aus sozial und wirtschaftlich marginalen Gruppen rekrutieren und dass Inzestfamilien hinsichtlich sozialer und persönlicher Probleme (Krankheit, beengte Wohnverhältnisse, Arbeitslosigkeit, Alkoholprobleme, reduzierte Intelligenz) in hohem Maße schon vor dem Auftreten inzestuöser Handlungen auffällig waren.

\section{„Natürliche” Inzestscheu?}

Der dem Bundesverfassungsgericht vorliegende Inzestfall ist dadurch gekennzeichnet, dass die Geschwister nicht zusammen aufgewachsen sind und sich erst im jungen Erwachsenenalter kennenlernten. Damit stellt sich die insbesondere auch schuldrelevante Frage, ob sich eine vor inzestuösen Handlungen schützende Inzestscheu bei den Geschwistern nicht entwickeln konnte. Die Frage, ob es „Inzestscheu“ auch beim Menschen gibt oder ob das Inzest-Tabu nur eine soziokulturell erlernte Norm darstellt, wird kontrovers diskutiert. Ausgangspunkt ist die „Westermarck“Hypothese, dass Menschen, die zusammen aufgewachsen sind, später eine gegenseitige, instinktive sexuelle Abneigung zueinander aufweisen (innate aversion). Hierbei liegt der entscheidende Faktor nicht in der genetischen Verwandtschaft. Der Inzest wird vielmehr durch das Heranwachsen in unmittelbarer Nähe zu Geschwistern (oder anderen Personen) verhindert. Inzestscheu funktioniert demnach dadurch, dass Geschwister, die in der frühen Kindheit engen körperlichen Kontakt hatten, sich in der Folge nicht als potentielle Sexualpartner, sondern als Geschwister definieren.

Beobachtungen, dass inzestuöse Beziehungen in der Tier- und Menschenwelt nicht die Regel, sondern Einzelfälle darstellen, führten zu der Hypothese einer genetischen Verankerung der Inzestscheu. Biologischer Hintergrund sei die Vermeidung von Inzucht und die Reduzierung des Risikos genetischer Probleme. Evolutionstheoretiker nehmen an, dass das neurale System des Menschen einen speziellen Erkennungsmechanismus (kin-recognition system) entwickelt hat, der es ihnen ermöglicht, (1) die Partnerwahl anhand der Theorie von Selektion und Fitness zu treffen und (2) sexuellen Kontakt zu genetisch verwandten Menschen zu hemmen, da die Nachkommen einer solchen Vereinigung eine größere Anzahl schädlicher genetischer Anlagen aufzeigen und die für die Anpassung notwendige genetische Variabilität einschränken könnten. Neuere Forschungen sprechen für die Wirksamkeit eines solchen Erkennungsmechanismus, der allerdings voraussetzt, dass Geschwister zusammen aufwachsen.

\section{Genetische Risiken}

Vor dem Hintergrund der Bedeutung einer Kontrolle genetischer Risiken für die Begründung des strafrechtlichen Inzestverbots erstreckte sich die Sekundäranalyse auf die Forschung zum Auftreten genetischer Defekte und damit zusammenhängender Krankheiten. Nachkommen inzestuöser Beziehungen weisen einen größeren Bestand homozygoter Gene auf, die im Gegensatz zu heterozygoten Genen durch eine geringere 
Variabilität gekennzeichnet sind. Ein hoher Grad an Heterozygotie bedeutet ein größeres Reaktionsvermögen auf variable Umweltbedingungen und somit eine höhere Vitalität. Bei Kindern aus inzestuösen Beziehungen ist diese Heterozygotie herabgesetzt. Selbst wenn es nicht zur konkreten Ausprägung einer Erberkrankung kommt, wird dadurch ein Verlust der allgemeinen Fitness (inbreeding depression) verursacht. Untersuchungen zu genetischen Risiken konzentrieren sich im Übrigen auf einige Weltregionen, in denen die Eheschließung zwischen nahen Verwandten sozialen Erwartungen entspricht.

Ein erhöhtes Risiko für das Auftreten genetisch bedingter Krankheiten im Vergleich zu Kindern aus nichtinzestuösen Beziehungen wird durch die Forschung belegt. Je enger das Verwandtschaftsverhältnis zwischen den Sexualpartnern, desto höher ist auch die Wahrscheinlichkeit einer genetisch bedingten Anomalie bei den Nachkommen. Genetische Erkrankungen und angeborene Abnormalitäten betreffen im Allgemeinen ca. 2 bis $5 \%$ aller Lebendgeburten und erklären etwa $50 \%$ der Sterbefälle von Kindern in westlichen Ländern. Eine Studie von 38 Populationen aus Asien, Afrika, Europa und Südamerika schätzt das Risiko einer Übertragung genetischer Anomalien bei Kindern aus inzestuösen Beziehungen um 1,7 bis 2,8\% höher ein im Vergleich zu nichtverwandten Verbindungen. Das Risiko für später auftretende Erkrankungen im ersten Lebensjahr liegt um bis zu 7 bis $31 \%$ höher im Vergleich zur normalen Population. Eine Untersuchung beobachtete in Jerusalem das Auftreten von genetischen Fehlbildungen bei arabischen Säuglingen aus inzestuösen Beziehungen. Sie kam zu dem Ergebnis, dass 8,7\% der aus inzestuösen Verbindungen Neugeborenen Fehlbildungen (z.B. Herzfehler, Down-Syndrom) aufwiesen, im Vergleich zu 2,6\% bei einer Kontrollgruppe. Auf der Grundlage der Untersuchungen lässt sich auf ein erhöhtes Risiko für genetische Schäden schließen. Freilich bleibt das Risiko in einer Größenordnung, die sich nicht weit vom durchschnittlichen Risiko der Normalpopulation entfernt.

\section{Geschwisterinzest und Strafverfolgung}

Die Beurteilung des strafrechtlichen Inzestverbots ist auch davon abhängig, wie und in welchem Grade das strafrechtliche Inzestverbot überhaupt implementiert wird. Die Strafverfolgungsstatistik unterscheidet allerdings nicht nach den einzelnen Inzestformen des $\$ 173$ StGB. Insgesamt nimmt die Zahl der Verurteilungen wegen $\$ 173$ StGB (insgesamt) in der zweiten Hälfte des 20. Jahrhunderts deutlich ab. Die Entwicklung belegt zum einen den Einfluss von Reformen des $\$ 173$ Anfang der 1970er Jahre. Sie belegt auch, dass die Strafverfolgung von Inzesthandlungen im langjährigen Durchschnitt zu unter 10 Verurteilungen pro Jahr führt. Davon dürften nur Einzelfälle den Geschwisterinzest betreffen, wie die Daten aus der Freiburger Kohortenuntersuchung zeigen. Vier Geburtskohorten (Baden-Württemberg, 1970, 1973, 1975, 1978) wurden auf polizeiliche und justizielle Registrierungen nach $§ 173$ Abs. 2 Satz 2 StGB hin ausgewertet. Bis heute liegen für diese Geburtskohorten (N: 3.600.000) 11 justizielle Erledigungen nach $§ 173$ Abs. 2 Satz 2 StGB vor. Die Erledigungen verteilen sich auf 20, 17, 15 und 12 Jahre Risikozeiträume der jeweiligen Geburtskohorten (ab einem Alter von 18 Jahren). Hieraus folgt, dass unter den heutigen Bedingungen aus einer Geburtskohorte in Deutschland bis zum Alter von etwa 35 Jahren maximal 3 bis 4 Personen wegen Geschwisterinzest registriert werden. $\$ 173$ Abs. 2 Satz 2 StGB spielt somit für die polizeiliche und justizielle Praxis sowie für die Struktur von Auffälligkeiten in den Geburtskohorten erwartungsgemäß keine Rolle. Nach den Daten der Strafverfolgungsstatistik handelt es sich ganz überwiegend um männliche erwachsene Verur-teilte. Vertiefende empirische Untersuchungen zu polizeilich oder justiziell registrierten Inzesttätern liegen aus neuerer Zeit nicht vor. Die Untersuchungen, die in der ersten Hälfte des 20. Jahrhunderts zu Inzestfällen durchgeführt worden sind, belegen, dass der Geschwisterinzest gegenüber dem Vater-Tochter-Inzest in der polizeilichen und justiziellen Praxis eine Ausnahme darstellt. Die Einzelfälle von Geschwisterinzest, die in diesen Untersuchungen identifiziert wurden, sprechen freilich für die Kombination „Älterer Bruder - Jüngere Schwester".

Zum Verfahrensablauf von Fällen des $\$ 173$ II StGB wurden in der ersten Hälfte des 20. Jahrhunderts vereinzelt empirische Untersuchungen zum Inzest (bzw. zur „Blutschande“) durchgeführt. Hier lag der Schwerpunkt des Forschungsinteresses freilich auf der Beschreibung der Täter und der Opfer, insbesondere auf deren sozialer Herkunft. Die empirischen Untersuchungen der gerichtlichen Erledigungen von Inzestfällen verweisen in dem relevanten Zeitraum insbesondere darauf, dass es sich um Straftaten aus sozial und wirtschaftlich randständigen Gruppen handelt. $\mathrm{Ob}$ es sich dabei um eine durch selektive Strafverfolgung ausgelöste Verzerrung zu Lasten unte- 
rer sozialer Schichten handelt, muss angesichts fehlender Daten zur Prävalenz des Inzests offen bleiben.

Die vorliegenden empirischen Untersuchungen enthalten keine Informationen zu den Gründen für Bekanntwerden der Fälle. Aus den Daten der Freiburger Kohortenuntersuchung lässt sich schließen, dass Fälle des $§ 173$ Abs. 2 Satz 2 StGB fast ausschließlich deshalb den Strafverfolgungsbehörden zur Kenntnis kommen, weil sich ein Opfer wegen gewalttätiger Übergriffe zur Anzeige entschlossen hat. Aus 13 Fällen, in denen $\$ 173$ Abs. 2 Satz 2 StGB angegeben wurde, resultieren 9, in denen die Strafverfolgung auch wegen Vergewaltigung oder sexuellen Missbrauchs $(\$ \S 177,176$ $\mathrm{StGB})$ eingeleitet worden ist. Die in der Freiburger Kohortenuntersuchung polizeilich erfassten Inzestfälle wurden überwiegend eingestellt (10 Fälle, davon 4 Einstellungen nach $\$ \$ 153,170$ StPO, 3 Verurteilungen, in drei Fällen war der Verfahrensausgang nicht bekannt). Die gerichtliche Erledigung von Inzestverfahren führt nach der Strafverfolgungsstatistik ganz überwiegend zu Geldstrafen.

\section{Fazit}

Zusammenfassend lässt sich feststellen, dass die Strafbarkeit des einverständlichen Inzests zwischen Erwachsenen nur wenige Fälle betrifft. Zwar liegen repräsentative empirische Untersuchungen zur Inzidenz und Prävalenz des Geschwisterinzests nicht vor. Doch belegen räumlich und von den erfassten Populationen her gesehen begrenzte Studien, dass sich Erfahrungen mit Geschwisterinzest auf etwa 2 bis $5 \%$ der untersuchten Gruppen beschränken. Ganz überwiegend handelt es sich dabei um einmalige und kurzfristige Beziehungen. Die meisten bekannt werdenden „Inzest“fälle enthalten weitere Umstände, aufgrund derer sie in allen Rechtsordnungen durch spezielle Tatbestände erfasst werden.

Im Fall einer Abschaffung der Inzestnorm würden sich daher in den meisten Ländern keine gravierenden Schutzlücken ergeben. In Deutschland werden jährlich etwa 10 Verurteilungen wegen Inzests registriert. Die Daten der Freiburger Kohortenuntersuchung kommen zu dem Ergebnis, dass bis zum Alter von 35 Jahren 3 bis 4 Personen eines Geburtsjahrgangs (mit einer knappen Million Angehörigen) wegen Inzeststraftaten justiziell registriert worden sind. Zudem hat sich gezeigt, dass das gesellschaftliche Inzesttabu auch in Rechtsordnungen ohne Inzestbestrafung erhalten bleibt. Bei der Erklärung einer „natürlichen“ Inzestscheu wird an genetische Grundlagen und an neuronal verankerte Erkennungsmechanismen gedacht. Die Inzestvermeidung ist freilich (sieht man von einigen Weltregionen ab, in denen bestimmte Formen der Verwandtschaftsehe sozial erwartet werden) so wirksam, dass sich sexuelle Beziehungen und die Heirat außerhalb von Verwandtschaftsverhältnissen entfalten. Ferner sind genetische Risiken erhöht; jedoch bleibt die Risikoerhöhung begrenzt. Genetischen Risiken kann im Übrigen mit Beratung begegnet werden. Daher ist eine Entkriminalisierung durchaus denkbar. Dies gilt insbesondere deshalb, weil Fallgestaltungen wie die vorliegende nicht nur sehr selten sind, sondern sich auch durch Besonderheiten auszeichnen, die eher dafür sprechen, dass eine „natürliche“ Inzestscheu wegen getrennten Aufwachsens nicht ausgebildet werden konnte.

\section{Die Entscheidung des Bundesverfassungsgerichts}

Die Entscheidung des Bundesverfassungsgerichts über die Inzeststrafbarkeit wurde am 26. Februar 2008 verkündet. Sie war ähnlich spektakulär wie der Fall von Patrick S. und Susan K. Eine Mehrheit von 7 Richtern hielt die Strafbarkeit des Geschwisterbeischlafs für verfassungsgemäß und überstimmte damit ihren Senatsvorsitzenden und Vizepräsidenten, der einer der bekanntesten Strafrechtler Deutschlands ist. Der Senatsvorsitzende wiederum kritisierte seine Kollegen in einem Sondervotum, in dem er die für ihn eindeutige Verfassungswidrigkeit der Norm begründete.
Nach der Mehrheitsentscheidung hat der Gesetzgeber im Hinblick auf die Begründung der Inzestnorm einen erheblichen Ermessensspielraum: „Es ist (...) grundsätzlich Sache des Gesetzgebers, den Bereich strafbaren Handelns verbindlich festzulegen. Er ist bei der Entscheidung, ob er ein bestimmtes Rechtsgut, dessen Schutz ihm wesentlich erscheint, gerade mit den Mitteln des Strafrechts verteidigen und wie er dies gegebenenfalls tun will, grundsätzlich frei." Als Schutzziel erkennt die Richtermehrheit die Verhinderung von familien- und sozialschädlichen 
Wirkungen an und billigt auch eugenische Verbotsbegründungen.

Nach dem überstimmten Senatsvorsitzenden und Vizepräsidenten des Gerichts schützt die Strafnorm dagegen kein anzuerkennendes Rechtsgut: Eine „nebulose kulturhistorisch begründete, wirkkräftige gesellschaftliche Überzeugung", wie sie vom Senat angenommen werde, könne eine Strafnorm nicht legitimieren. Und insbesondere zur genetischen Strafbegründung: „Der Gedanke eines strafrechtlichen Schutzes potentieller Nachkommen vor genetischen Schäden setzt (... ) die absurde Abwägung des mutmaßlichen Interesses potentiell gezeugten Nachwuchses an einem Leben mit genetischen Defekten einerseits mit einem mutmaßlichen Interesse an der eigenen Nichtexistenz andererseits voraus." Beide Lager berufen sich dabei mehrfach auf die vom Max-Planck-Institut erhobenen Befunde.

Eine Abschaffung der Strafnorm könnte damit nur noch durch den Gesetzgeber erfolgen. Auch für eine solche Entscheidung sind strafrechtliche und kriminologische Erkenntnisse relevant. Unter strafrechtlichen Gesichtspunkten wirft das Urteil vor allem die Frage auf, ob bei einem Strafgesetz wie bei jedem anderen Grundrechtseingriff nur nach der Verhältnismäßigkeit zu fragen ist, d.h. nach einem legitimen Eingriffszweck, nach der Erforderlichkeit und nach der Verhältnismäßigkeit im engeren Sinne. Die klassische Strafrechts- theorie scheint demgegenüber mit der Forderung nach dem Schutz eines bestimmten Rechtsguts sowie nach dem fragmentarischen Einsatz des Strafrechts als ultima ratio höhere Anforderungen zu verlangen.

Damit geht es in dem vorliegenden Fall nicht nur um inzestspezifische Probleme, sondern vor allem auch um die Frage, ob das Strafrecht mit seinem sozialethischen Vorwurf und seinen besonders eingriffsintensiven freiheitsentziehenden Maßnahmen strengeren Maßstäben und damit anderen Grenzen unterliegt als Maßnahmen in anderen Gebieten des öffentlichen Rechts. Diese Fragen sind grundlegend und auch in den anderen oben dargestellten Beiträgen relevant, in denen z.B. bei der Verfolgung des Terrorismus vom Strafrecht auf das Polizei- oder das Ausländerrecht ausgewichen wird.

Die strafrechtliche und die kriminologische Forschungsabteilung des Freiburger Max-PlanckInstituts werden deswegen nicht nur ihre gutachterliche Stellungnahme im Rahmen eines erweiterten Forschungsprojekts veröffentlichen, sondern vor allem auch weiter über diese Grundlagenfragen nach den Grenzen des Strafrechts in einer sich wandelnden Welt arbeiten.

Hans-Jörg Albrecht, Ulrich Sieber, Konstanze Jarvers 
III. Nachwuchsförderung 
III. NACHWUCHSFÖRDERUNG

A. Überblick

B. Ausbildungsbeginn in der International Max Planck Research School for Comparative Criminal Law

C. Vorbereitung der International Max Planck Research School on Retaliation, Mediation and Punishment

D. Beteiligung an der MaxNetAging Research School 


\section{A. Überblick}

Die am Institut forschenden Doktoranden werden insbesondere von den Direktoren des Instituts in den für die strafrechtliche und kriminologische Forschung wesentlichen Disziplinen betreut (Rechtswissenschaften, Soziologie, Psychologie). Die gute Zusammenarbeit mit der Rechtswissenschaftlichen Fakultät der Universität Freiburg bei der Ausbildung des wissenschaftlichen Nachwuchses wird seit 2007 durch die Internationale Max Planck Research School für Strafrechtsvergleichung (IMPRS-CC) und seit 2008 weiter durch die International Max Planck Research School on Retaliation, Mediation and Punishment (IMPRS-REMEP) vertieft.

Die Doktoranden promovieren in der Regel zum Dr. jur. Dies gilt nicht nur für die strafrechtliche Forschungsabteilung, sondern auch für die kriminologische Abteilung, da die Kriminologie - wie an fast allen deutschen Universitäten - zur Rechtswissenschaftlichen Fakultät der Albert-Ludwigs-Universität Freiburg gehört. Abhängig von dem jeweiligen akademischen Abschluss können Doktoranden auch an der Philosophischen und der Verhaltenswissenschaftlichen Fakultät promovieren. Eine Zulassung an der Universität Freiburg ist jedoch nicht zwingend erforderlich. So können auch Doktoranden mit einer Zulassung an anderen deutschen Universitäten betreut werden, wenn einer der Betreuer dort ein Prüfungsrecht hat (wie vorliegend z.B. an der Ludwig-Maximilians-Universität München). Doktoranden können auch an anderen Universitäten zugelassen sein, mit denen das Institut zum Zweck der Nachwuchsförderung kooperiert.

In der strafrechtlichen Forschungsabteilung ist die Zahl der Doktoranden im Berichtszeitraum 2006/2007 durch den Ende 2003 erfolgten Direktorenwechsel geprägt. Der neue Direktor der strafrechtlichen Forschungsabteilung betreute im Berichtszeitraum 16 Doktoranden, davon 11 deutsche und 4 ausländische aus Bosnien-Herzegowina, China, Kanada und der Schweiz. Davon wurden 2 Dissertationsverfahren im Berichtszeitraum und ein weiteres Anfang 2008 abgeschlossen. Für das Jahr 2008 ist bereits mit 4 weiteren Doktoranden aus Russland, Kolumbien, der Schweiz und Deutschland eine Betreuung durch den Direktor der strafrechtlichen Abteilung vereinbart. Hinzu kommen im Strafrecht auch die 9 von anderen Professoren in der Internationalen Max Planck Research School für Strafrechtsvergleichung betreuten Doktoranden sowie 3 weitere Dissertationsverfahren von Mitarbeiterinnen und Mitarbeitern bei externen Hochschullehrern. Der emeritierte Institutsdirektor betreute im Berichtszeitraum eine Dissertation, die abgeschlossen wurde. Die Förderung des wissenschaftlichen Nachwuchses ist in der strafrechtlichen Abteilung grundsätzlich in das neue strafrechtliche Forschungsprogramm eingebunden. Die Schwerpunkte liegen deswegen auf den territorialen und funktionalen Grenzen des Strafrechts, vor allem in den Deliktsbereichen der Internetkriminalität, der Wirtschaftskriminalität, der organisierten Kriminalität und des Terrorismus. Die Themenstellungen werden dabei anhand der nationalen Rechtsordnungen sowie anhand des Europäischen Strafrechts und des Völkerstrafrechts - zu einem großen Teil rechtsvergleichend - behandelt. In Zukunft wird die Nachwuchsausbildung zunehmend in die beiden vom Institut geleiteten Research Schools verlagert.

In der kriminologischen Abteilung wurden im Berichtszeitraum 2006/2007 insgesamt 42 Doktoranden betreut, davon 39 vom Direktor der kriminologischen Abteilung. Die Doktoranden kamen aus Deutschland, Griechenland, Zentral- und Ostasien (Volksrepublik China, Republik China, Korea, Mongolei), dem Nahen Osten (Iran, Türkei, West Bank und Gaza) sowie Lateinamerika (Argentinien, Bolivien, Chile, Venezuela). Dabei liegen die Forschungsschwerpunkte „strafrechtliche Sanktionen“, „organisierte Kriminalität, Terrorismus und Innere Sicherheit“ sowie „empirische Strafverfahrensforschung“ im Mittelpunkt der Nachwuchsförderung. Im Berichtszeitraum wurden 7 Dissertationen abgeschlossen. Eine Liste der Doktoranden aus beiden Abteilungen ist im Anhang abgedruckt. 


\section{B. Ausbildungsbeginn in der International Max Planck Research School for Comparative Criminal Law}

Im Frühjahr 2007 wurde - vor allem von der strafrechtlichen Forschungsabteilung - die erste International Max Planck Research School des Instituts gegründet, die International Max Planck Research School for Comparative Criminal Law (IMPRSCC). Das auf zwei Jahre ausgerichtete Ausbildungsprogramm wird vom Max-Planck-Institut für ausländisches und internationales Strafrecht und der Rechtswissenschaftlichen Fakultät der AlbertLudwigs-Universität Freiburg getragen. Sprecher der Research School ist Prof. Dr. Dr. h.c. Ulrich Sieber; stellvertretender Sprecher Prof. Dr. Walter Perron, Dekan der Rechtswissenschaftlichen Fakultät der Universität Freiburg. Neben ihnen sind Mitglieder des Lenkungsausschusses und Betreuer auch Prof. Dr. Dr. h.c. Hans-Jörg Albrecht, Direktor am Max-Planck-Institut für ausländisches und internationales Strafrecht, sowie Prof. Dr. Wolfgang Frisch und Prof. Dr. Roland Hefendehl von der Universität Freiburg. Koordinator der Research School ist Jan-Michael Simon. Der erste Jahrgang der IMPRS-CC besteht aus derzeit zehn ausländischen (Bosnien-Herzegowina, Chile, China, Estland, Iran, Polen, Schweiz) und sieben deutschen Doktoranden. Für das Jahr 2008 ist die Aufnahme von vier weiteren Doktoranden (Deutschland, Schweiz, Russland, Kolumbien) vorbereitet.

Die IMPRS-CC fördert und verzahnt im Rahmen eines übergreifenden Forschungsprogramms zur Strafrechtsvergleichung bis zu 20 Doktorarbeiten und trägt auch darüber hinaus zur Ausbildung des wissenschaftlichen Nachwuchses bei. Der Forschungsgegenstand und die Ziele der Research School werden dabei vor allem durch das Forschungsprogramm der strafrechtlichen Abteilung des Instituts bestimmt, insbesondere durch dessen Forschungsschwerpunkt zur Strafrechtsvergleichung. Dabei geht es um ein Strafrecht zum Schutz der Gesellschaft und um eine menschenwürdige, demokratische und rechtsstaatliche Kriminalpolitik im Kontext zunehmend weltumspannender Interaktion („Globalisierung“) und der Veränderung von Risiken („Risikogesellschaft“ und „Informationsgesellschaft"). Im Mittelpunkt des Forschungsprogramms stehen daher die Harmonisierung und die Internationalisierung von Strafrecht, seine in- ternationale Institutionalisierung sowie seine territorialen und funktionalen Grenzen. Insoweit kann auf die Ausführungen oben in Kapitel I.B. zum Forschungsprogramm der strafrechtlichen Abteilung verwiesen werden.

Ziel des Forschungsprogramms ist es vor allem, auf der Grundlage von neuen Erkenntnissen über die Kriminalität und ihre Entwicklung neues Wissen über den Grad an Konvergenz und Divergenz zwischen Strafrechtsordnungen sowie über die Kontrollmöglichkeiten, Grenzen und Entwicklungstendenzen des Strafrechts zu schaffen. Darüber hinaus geht es im Kontext der Globalisierung um andere Strategien der Kriminalitätskontrolle als das Strafrecht. Damit wird die Grundlage für Modelle und Lösungen in der Kriminalpolitik und für die Rechtsanwendung geschaffen. Methodisch ist dafür - neben der Analyse der Rechtstatsachen - vor allem eine universale und funktionale Strafrechtsvergleichung erforderlich, die vor dem Hintergrund unterschiedlicher gesellschaftlicher Bedingungen und philosophischer Traditionen die strukturellen Zusammenhänge von Strafrecht untersucht.

Der internationale Schwerpunkt des Forschungsprogramms, der globale Kontext seines Forschungsgegenstandes und der universale Ansatz der Strafrechtsvergleichung vermitteln den Doktoranden ein besseres Verständnis für und eine kritische Distanz zu der eigenen Rechtsordnung, die Sensibilität für die zunehmende internationale Annäherung und Anerkennung unterschiedlicher rechts- und kriminalpolitischer Standpunkte sowie die Fähigkeit zur Entwicklung übergreifender Regelungen, die sowohl Unterschiede zwischen wie Gemeinsamkeiten von nationalen Strafrechtsordnungen einbeziehen. Diese inhaltlichen Aspekte der Ausbildung werden durch ausgesuchte Ausbildungseinheiten zu grundlegenden und spezifischen Aspekten der Strafrechtsvergleichung sowie zu allgemeinen Schlüsselqualifikationen und zur eigenständigen, problemorientierten und verantwortungsbewussten Forschung ergänzt.

Die forschungsorientierte Ausrichtung des Curriculums legt den Schwerpunkt der Ausbildung auf die 


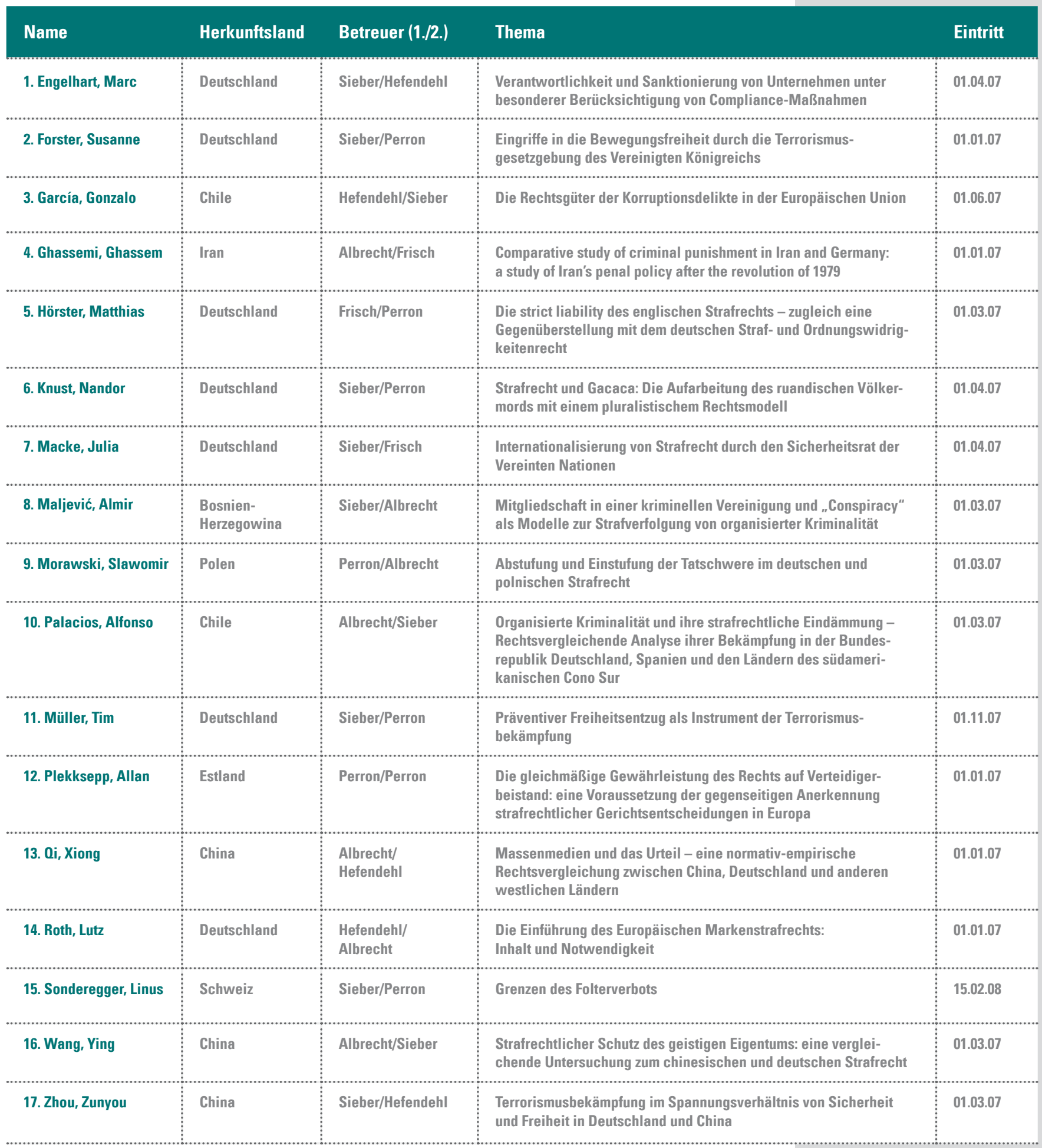

monatlichen Doktorandenkolloquien der IMPRSCC. Seit Beginn der Research School wurden 13 Doktorandenkolloquien durchgeführt, an denen in der Regel zwei Doktorarbeiten vorgestellt und diskutiert werden. Weiter wurde im November 2007 das erste, einmal jährlich erfolgende Blockseminar zu den Grundlagen der Strafrechtsvergleichung veranstaltet. Ferner nahmen die Doktoranden an wis- senschaftlichen Veranstaltungen in- und außerhalb des Hauses teil. Die Doktoranden waren teilweise in Forschungsprojekte des Instituts eingebunden. Über die regelmäßig stattfindenden Betreuungsgespräche mit dem Erstbetreuer hinaus führte jeder Doktorand im Berichtszeitraum zwei Betreuungsgespräche mit seinem Betreuungsausschuss (bestehend aus dem Erst- und Zweitbetreuer).
Tabelle:

Übersicht über die Dissertationen in der Max Planck Research School for Comparative Criminal Law 


\section{Vorbereitung der International Max Planck Research School on Retaliation, Mediation and Punishment}

Im Berichtszeitraum wurde - vor allem für die Arbeiten der kriminologischen Abteilung - die International Max Planck Research School on Retaliation, Mediation and Punishment (IMPRSREMEP) vorbereitet, die ihr Programm am 1. April 2008 aufgenommen hat. Das auf grundsätzlich zwei Jahre ausgerichtete Ausbildungsprogramm wird gemeinsam getragen von den beiden Abteilungen des Max-Planck-Instituts für ausländisches und internationales Strafrecht, dem Max-PlanckInstitut für ausländisches öffentliches Recht und Völkerrecht (Heidelberg), dem Max-Planck-Institut für ethnologische Forschung (Halle) und dem Max-Planck-Institut für europäische Rechtsgeschichte (Frankfurt), der Rechtswissenschaftlichen Fakultät der Albert-Ludwigs-Universität Freiburg und der Philosophischen Fakultät I der Martin-Luther-Universität Halle-Wittenberg. Ihr Sprecher ist Prof. Dr. Dr. h.c. Hans-Jörg Albrecht, ihr stellvertretender Sprecher Prof. Dr. Günther Schlee, Direktor am Max-Planck-Institut für ethnologische Forschung in Halle, und ihre Koordinatorin Dr. Carolin Hillemanns.

REMEP richtet sich an Nachwuchswissenschaftler aus den Rechtswissenschaften, der Kriminologie, der Rechtsgeschichte, des Völkerrechts sowie der Sozial- und Rechtsanthropologie. Es bietet bis zu 20 in- und ausländischen Doktoranden während maximal drei Jahren die Möglichkeit, interdisziplinär zum Themenbereich Vergeltung, Mediation und Bestrafung in einem Verbund von Max-Planck-Instituten und Hochschulen zu forschen. Die Doktoranden können an ihren jeweiligen Standorten (Frankfurt, Freiburg, Halle, Heidelberg) die hervorragenden Forschungsmöglichkeiten der Institute nutzen. Sie werden fächerübergreifend von Direktoren sowie Hochschulprofessoren im Rahmen von sogenannten Thesis Committees bei ihrer Forschungsarbeit betreut. In einem eigens hierfür aufgebauten, strukturierten und interdisziplinären Trainingsprogramm in Form von Kolloquien und Workshops werden mehrmals pro Jahr Blockveranstaltungen angeboten, die zu dem Pflichtprogramm gehören, an dem alle Doktoranden der REMEP teilnehmen. Die Kolloquien werden abwechselnd an allen vier Standorten abgehalten. Ziel der Kolloquien ist, dass sich die Doktoranden über ihr eigenes Forschungsprojekt hinaus mit den theoretischen Grundlagen und empirischen Zugängen aller beteiligten Disziplinen auseinandersetzen. Darüber hinaus sollen sogenannte Soft Skills vermittelt und die soziale Integration der Doktoranden gefördert werden. Die Trainingssprache ist Englisch.

Im Mittelpunkt des Forschungsprogramms steht die Frage nach dem Verhältnis von Frieden und sozialer Ordnung. Dies ist vor dem Hintergrund zu sehen, dass seit der Entstehung des modernen Nationalstaats dieser das Gewaltmonopol und als eines seiner stärksten Ausformungen das Recht zur strafrechtlichen Sanktionierung innehat. Andere, zum Teil tradierte Formen der Konfliktlösung wie beispielsweise Mediation oder auch private Vergeltung wurden hier zurückgedrängt. Es zeigt sich aber heute in vielfältiger Weise, dass diese grundsätzlich positiv zu beurteilende Errungenschaft einer Konzentration der Gewalt im Staate in mancher Hinsicht eine unzureichende Entwicklung war und/oder nicht mit dem gewünschten Erfolg in alle Teilbereiche des Lebens hineinreichen kann (z.B. innerhalb einer Gruppe von Einwanderern, die keinen Zugang zur staatlichen Konfliktregelung findet oder sucht oder auch will, z.B. die Familie) und auch deshalb zunehmend auf alternative Formen der Konfliktlösung zurückgegriffen wird (z.B. Opfer-Täter-Ausgleich, sog. „,Truth and Reconciliation Commissions", Selbstregulierung der Wirtschaft etc.). Im Lauf der vergangenen Jahre wurde deutlich, dass lediglich ein interdisziplinärer Forschungsansatz neue Antworten geben kann auf die Frage nach dem Verhältnis von Frieden und sozialer Ordnung. Dies soll durch die Research School geleistet werden.

Im Rahmen ihres Forschungsprogramms soll deswegen den zentralen Fragestellungen der Aushandlung, Konstruktion, Aufrechterhaltung und Wiedergewinnung sozialer Ordnung und sozialer Kontrolle nachgegangen werden. Diese Grundsatzfragen haben gerade auch im Kontext von Konflikt- und Postkonfliktgesellschaften neue Bedeutung gewonnen, in denen bei der Suche 
nach modernen Lösungsstrategien auch tradierte Ansätze der Konfliktregelung (Mediation) nutzbar gemacht werden. Diese treten - partiell - neben überkommene Vergeltungskonzepte und ergänzen die etablierten Modelle des Strafens und der Strafbegründung oder ersetzen sie teilweise ganz. Die Herangehensweise an diese zentralen Fragestellungen erfolgt jeweils aus der unterschiedlichen Perspektive der beteiligten Fachrichtungen, wobei aber ein interdisziplinärer Ansatz gewollt ist. In Freiburg soll der Schwerpunkt auf Studien liegen, die sich im weitesten Sinne der kriminologischen bzw. strafrechtlichen und strafrechtsvergleichenden Forschung zuordnen lassen. Ein Bezug zu der Grundfrage, welchen Beitrag die Existenz bzw. Abwesenheit von Strafrecht bei der Entstehung, Wiederentstehung oder dem Zerfall gesellschaftlicher Ordnung spielt, auf welche Formen und Ausprägungen strafrechtlicher Sozialkontrolle zurückgegriffen wird und welche Substitute (formalisierter) Strafrechtskontrolle denkbar sind, sollte bei den Forschungsarbeiten stets erkennbar sein.

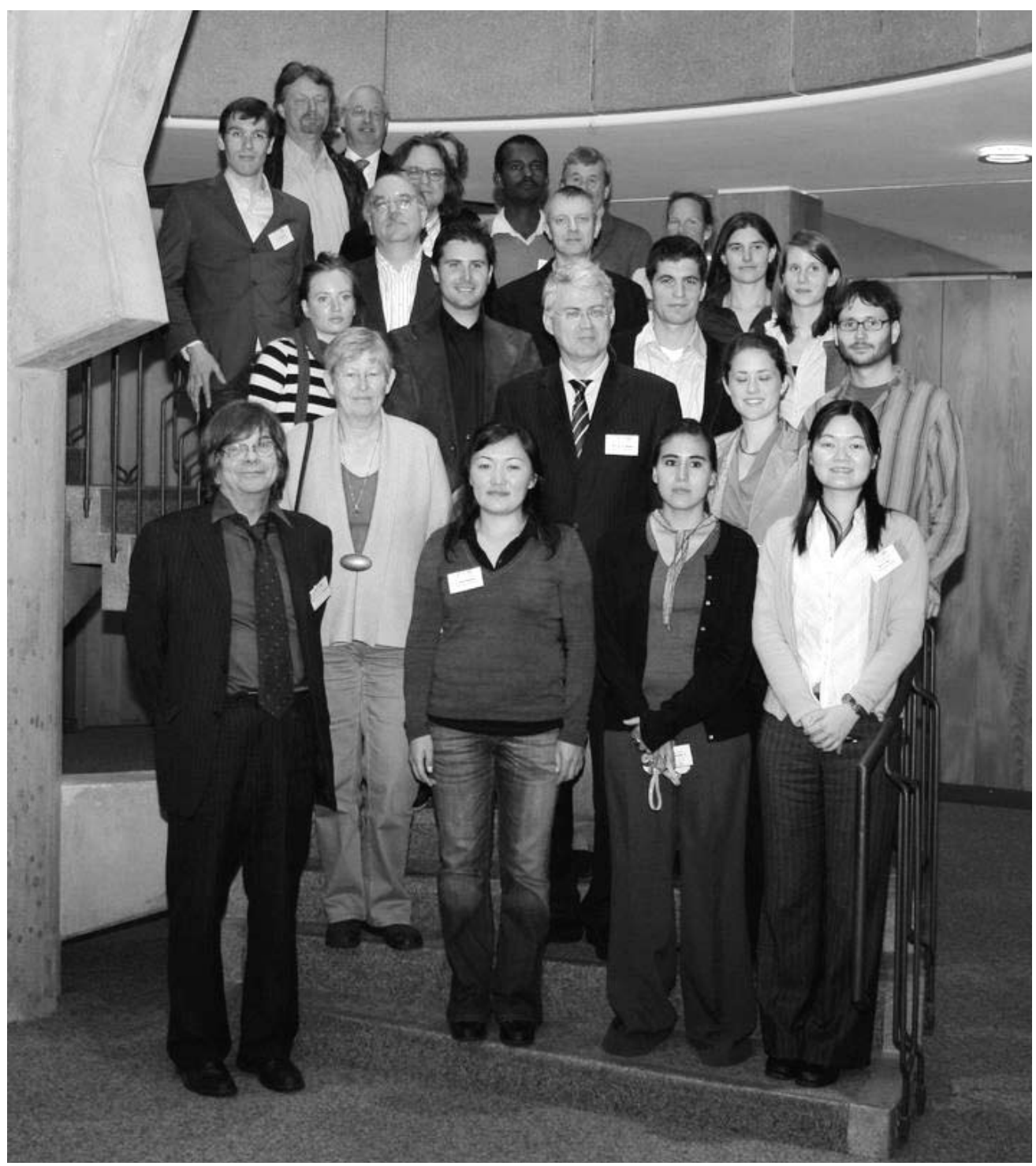

Doktorandinnen und Doktoranden der 2008 beginnenden Max Planck Research School on Retaliation, Mediation and Punishment mit ihren Betreuern 


\section{Beteiligung an der MaxNetAging Research School}

Die institutsübergreifende MaxNetAging Research School (MNARS) ist Teil des Max Planck International Research Network on Aging (MaxNet Aging). Gegenstand dieses internationalen Netzwerks sind die Ursachen, Prozesse und Konsequenzen des Alterns. Kooperationspartner des Netzwerks sind 12 Max-Planck-Institute sowie amerikanische und schwedische Forschungseinrichtungen, die Politikwissenschaften, Soziologie, Anthropologie, Rechtswissenschaften, Ökonomie, Geschichtswissenschaften, Demographie, Mathematik, Biologie, Medizin, Psychologie und die Gehirnwissenschaften in einem interdisziplinären Forschungsverbund vereinigen. MaxNetAging wurde von Prof. Dr. Paul B. Baltes (1939-2006) gegründet und steht seit 2007 unter der Leitung von Prof. Dr. James W. Vaupel (Max-Planck-Institut für demographische Forschung, Rostock).

MNARS wurde 2007 als Ausbildungskomponente von MaxNetAging gegründet und zunächst auf drei Jahre befristetet. Insgesamt nehmen 12 Doktoranden aus den unterschiedlichen Fachrichtungen der 12 beteiligten Max-Planck-Institute teil. Das neunmonatige Ausbildungsprogramm wird am Max-Planck-Institut für demographische Forschung durchgeführt. Ziel des Ausbildungsprogramms ist es, die Doktoranden in eine interdisziplinäre Forschungsperspektive zu Prozessen und Konsequenzen des Alterns einzuführen, um auf diese Weise interdisziplinäre Forschungsansätze auf einer soliden methodischen, theoretischen und empirischen Grundlage zu dem vielschichtigen Forschungsstand von MNARS zu stimulieren. Das Ausbildungsprogramm besteht aus wöchentlichen Vorträgen mit anschließenden Kolloquien, Einführungskursen in Forschungsmethoden, einem Forschungsseminar zu grundlegenden Ansätzen in der Altersforschung sowie einer Graduiertenklasse. In der Graduiertenklasse stellen die Doktoranden ihr Dissertationsprojekt zur Diskussion. Nach Beendigung des Ausbildungsprogramms werden die Forschungsarbeiten an den jeweiligen Max-PlanckInstituten weitergeführt.

Frau Franziska Kunz (Soziologin) hat am Freiburger Max-Planck-Institut für ausländisches und internationales Strafrecht am 1. November 2007 im Rahmen der MNARS eine Untersuchung zu „Devianz und Kriminalität im Alter“ begonnen. Die von Prof. Dr. Albrecht und Prof. Dr. Blinkert betreute Studie „Eine theoretische und empirische Studie zu den Bedingungen von Devianz und Kriminalität in fortgeschrittenen Altersphasen" soll aus einer theoretischen und empirischen Perspektive die bislang vernachlässigten devianzsoziologischen und kriminologischen Phänomene der Alterskriminalität aufgreifen. Im Zentrum steht die Entwicklung einer spezifischen Theorie der Alterskriminalität. 


\section{Wissenschaftliche Zusammenarbeit}


IV. WISSENSCHAFTLICHE ZUSAMMENARBEIT

A. Internationale und nationale Kooperation

B. Ausländische Wissenschaftler am Institut

C. Gutachten

D. Veranstaltungen und Vorträge

E. Lehre 


\section{A. Internationale und nationale Kooperation}

\section{Internationale Kooperationen}

Das Institut pflegt weltweit enge Verbindungen zu ausländischen Forschungseinrichtungen und Wissenschaftlern sowie Akteuren und Institutionen aus Politik und Praxis. Der Schwerpunkt der internationalen Kooperationen beruht dabei auf fünf forschungsstrategischen Zielen:

1. Systematische Erweiterung und Konzentration von Informationen zu den Schwerpunkten beider Forschungsprogramme durch Kooperationsprojekte weltweit.

2. Aufbau und nachhaltige Pflege eines internationalen Netzwerks exzellenter Forschungseinrichtungen und Forscher.

3. Förderung des kritischen Diskurses zu den Schwerpunkten der Forschungsprogramme durch internationalen Austausch mit Wissenschaft, Politik und Praxis.

4. Förderung der Synergien zwischen Grundlagenforschung und der Ausbildung von Nachwuchswissenschaftlern aus der ganzen Welt.

5. Unterstützung von Entwicklungsländern und Übergangsgesellschaften beim Aufbau eines modernen, rechtsstaatlichen Strafrechts und Justizsystems.

Geographisch decken die Kooperationen alle wichtigen Regionen ab. Ein europäischer Schwerpunkt ergibt sich dabei sowohl aus der gewachsenen $\mathrm{Zu}$ ständigkeit der Europäischen Union in den Bereichen Kriminalitätsprävention und Strafrechtsharmonisierung als auch aus der zunehmenden Bedeutung der europäischen Forschungsförderung. Weitere Schwerpunktbildungen betreffen den Nahen und Mittleren Osten, die Volksrepublik China sowie Lateinamerika.

Unter aktuellen Gesichtspunkten des Berichtszeitraumes lässt sich die internationale Zusammenarbeit wie folgt darstellen:

Das Institut arbeitet gegenwärtig an einer umfassenden und thematisch breit angelegten rechtsvergleichenden Untersuchung über die allgemeinen Grundsätze und Fragen des Strafrechts, wobei das Pilotprojekt zu zwölf Rechtsordnungen ab 2008 auf weitere Staaten ausgedehnt werden soll. Eine spezielle Projektgruppe untersuchte normative Fragen der „Participation in Crime: Criminal Liability of Leaders of Criminal Groups and Networks" mit einem internationalen Netzwerk von ca. 40 Partnern, wobei auch das Internationale Straftribunal für das ehemalige Jugoslawien (ICTY) beteiligt war. Ein in Zusammenarbeit mit der Europäischen Kommission durchgeführte Projekt über die Grundlagen und die Reform des Europäischen Strafrechts, mit dem die Strukturmodelle zur Strafverfolgung transnationaler Kriminalität in der EU analysiert werden, beruht auf der Rechtsvergleichung von 19 Rechtsordnungen und bezieht auch die USA ein.

Im rechtsvergleichenden Projekt „Les chemins de l'harmonisation pénale“ (Universität Basel, Collège de France, Universidad de Castilla-La Mancha) werden die Akteure, Wirkkräfte und Modelle der internationalen Strafrechtsharmonisierung in Zusammenarbeit von Forschern aus verschiedenen europäischen Staaten untersucht.

Mit dem Drug Policy Research Center/RAND wird eine Wirkungsstudie zu „Effekten der Cannabis-Depönalisierung in westlichen Industriestaaten" umgesetzt. In Zusammenarbeit mit der Universität Mailand und der EU-Bebachtungsstelle für Drogen werden die Strukturen der Drogenmärkte in Frankfurt und Mailand analysiert. Aus dem bis 2009 laufenden Laboratoire Européen Associé (LEA, MPI, CESDIP Paris, Universität Lille) wird eine Studie zu „Diskriminierung und Polizei“ in Großstädten Deutschlands und Frankreichs (Paris, Berlin) durchgeführt. Zusammen mit französischen Forschungsinstituten wird eine Untersuchung zur Rolle der Polizei in ethnisierten Konflikten vorbereitet.

Auf der Zusammenarbeit mit der Berliner Humboldt-Universität und einem Netzwerk von Forschungseinrichtungen aus verschiedenen Weltregionen beruht eine Forschungsinitiative zu „Retaliation, Mediation and Punishment“. Die Initiative untersucht die Funktion von Vergeltung, 


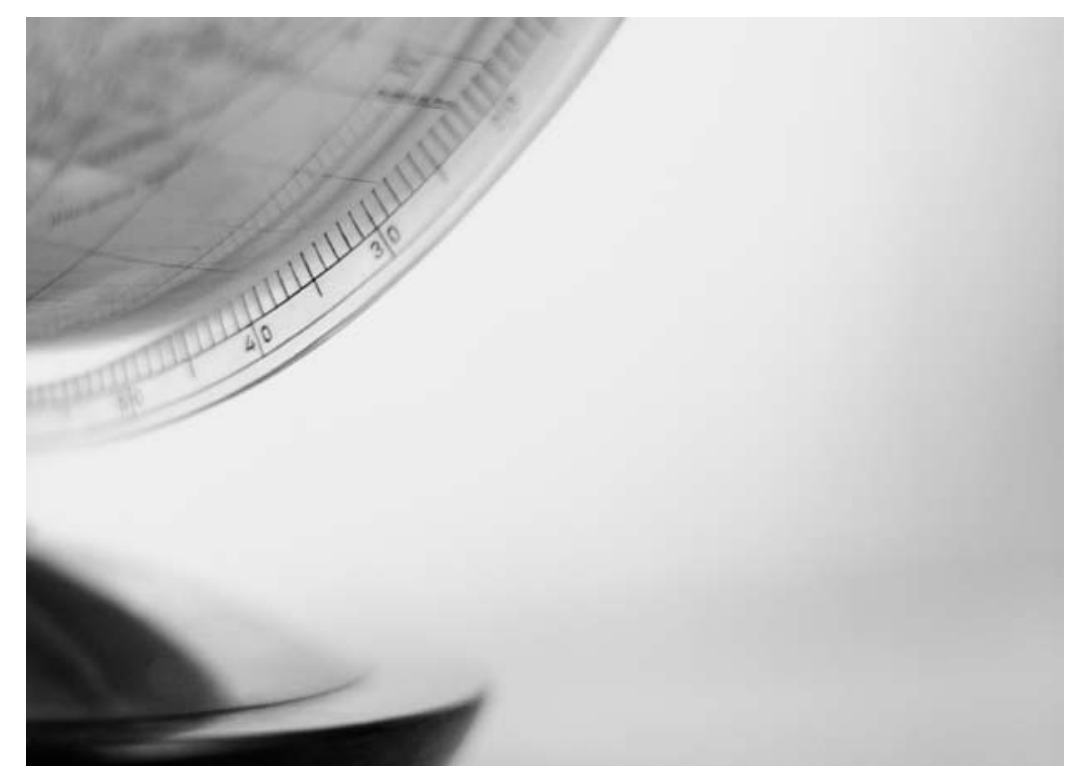

Mediation und staatlicher Strafe in der Herstellung von sozialer Ordnung unter verschiedenen kulturellen und ökonomischen Bedingungen. Eine im Jahr 2007 begonnene und durch die Europäische Union geförderte vergleichende Untersuchung zum erweiterten Suizid schließt Forschungseinrichtungen aus mehreren europäischen Ländern ein.

In einer Anfang 2006 begonnenen und unter Beteiligung des Great Britain China Center (London) und der Universität Essex durchgeführten Untersuchung zur Implementation der Anti-Folter-Konvention der Vereinten Nationen ist auf chinesischer Seite die Renmin University eingebunden. Unter Beteiligung des Great Britain China Center (London), der Beijing Normal University, der Wuhan

\section{Nationale Kooperationen}

Im Mittelpunkt nationaler Kooperationen steht die Zusammenarbeit mit Universitäten.

Prof. Dr. Hans-Jörg Albrecht und Prof. Dr. Ulrich Sieber haben an der Juristischen Fakultät der Albert-Ludwigs-Universität Freiburg den Status eines „qualifizierten Honorarprofessors“ mit vollen Mitwirkungsrechten in der Fakultät. Herr Albrecht ist ferner Mitglied der Philosophischen Fakultät. Herr Sieber ist auch Honorarprofessor und Mitglied der Juristischen Fakultät der Ludwig-Maximilians-Universität München.
University und der Oxford University (Centre of Criminology) wird seit 2006 ein Projekt zum Thema „Todesstrafe und öffentliche Meinung in China" durchgeführt.

In einer Zusammenarbeit mit der Universität Tilburg begann eine empirische Untersuchung zur „Radikalisierung junger Menschen in Europa“. Aus der im Jahr 2006 zwischen dem Institut, dem Niederländischen Zentrum für Forschungen zu Kriminalität und Strafjustiz, der University of Cambridge sowie der Universität Tübingen vereinbarten dauerhaften wissenschaftlichen Kooperation (European Network of Research Institutes of Criminology - ENRIC) gingen im Jahr 2007 zwei Wokshops hervor.

Prof. Dr. Wolfgang Frisch und Prof. Dr. Wolfgang Perron, beide Universität Freiburg, wurden 2005 zu Auswärtigen Wissenschaftlichen Mitgliedern des Max-Planck-Instituts für ausländisches und internationales Strafrecht berufen. Dabei steht die Berufung von Herrn Perron auch in Zusammenhang mit der im Jahr 2000 getroffenen Kooperationsvereinbarung zwischen der Freiburger Universität und der Max-Planck-Gesellschaft. Diese sieht für den Inhaber des Lehrstuhls für Strafrecht, Strafprozessrecht und Strafrechtsvergleichung den Status eines Auswärtigen Wissen- 
schaftlichen Mitglieds am MPI vor. Umgekehrt ist geregelt, dass der am Max-Planck-Institut für die strafrechtliche Abteilung zuständige Direktor zum qualifizierten Honorarprofessor an der Juristischen Fakultät der Universität Freiburg berufen wird.

Prof. Dr. Jörg Arnold ist Honorarprofessor an der Universität Münster und lehrt dort auch über die Themen seiner Forschung am Max-Planck-Institut.

Das Max-Planck-Institut für ausländisches und internationales Strafrecht ist in Zusammenarbeit mit der Juristischen Fakultät Freiburg, den Universitäten München, Frankfurt, Köln und Hamburg am Aufbau des Instituts für Deutsches Recht an der China Universität für Politische Wissenschaften und Recht in Peking beteiligt. Im Übrigen ist das Max-Planck-Institut in das Kon-

\section{Perspektiven}

Die Forschungsperspektiven beziehen ihre Koordinaten aus allgemeinen Entwicklungstrends, die in den Begriffen der Globalisierung sowie der Risiko- und der Informationsgesellschaft zum Ausdruck kommen. Hieraus ergeben sich Fragestellungen zu den Grenzen der strafrechtlichen Sozialkontrolle in einer Welt, die durch neue und grenzüberschreitende Risiken, neue Informationstechnologien, Migration und hierdurch entstehende Konfliktpotentiale sowie eine zunehmende Bedeutung internationaler und supranationaler sortium zum Aufbau eines „Instituts für Europäisches Recht" in Peking eingebunden (Leitung: Universität Hamburg).

Das Institut führt ein interdisziplinäres und die Fachbereiche der Philosophie und der Anglistik an der Universität Freiburg einschließendes DFG-Verbundprojekt zu „Recht, Norm, Kriminalisierung" durch und nimmt seit Ende 2007 an einem Verbund zur Sicherheitsforschung (Fraunhofer Institut Karlsruhe, Institut für Soziologie an der Universität Freiburg) teil. Zusammen mit der Universität Göttingen wird eine Rückfallstatistik auf der Grundlage des Bundeszentralregisters erstellt. Das Institut kooperiert außerdem mit der technischen Universität Dresden in einer Untersuchung über die technische Sperrung von illegalen Inhalten im Internet.

Standards (insb. Menschenrechte) gekennzeichnet ist. Gleichzeitig erhöht der Prozess der Globalisierung den Bedarf an international einordnungsfähigem Wissen und an funktionsfähigen Netzwerken der Forschung. Er fordert schließlich eine komparative Methodologie, um die Entwicklungen in grenzüberschreitenden Fragen, in nationalen Strafrechtssystemen und auf supranationaler (internationaler und europäischer) Ebene abbilden können. 


\section{B. Ausländische Wissenschaftler am Institut}

Das Freiburger Max-Planck-Institut für ausländisches und internationales Strafrecht ist mit seinem interdisziplinären und rechtsvergleichenden Forschungsprogramm ein Anziehungspunkt für Wissenschaftler/innen aus aller Welt. Es bietet ihnen die Möglichkeit, in einem zeitlich befristeten Rahmen individuelle Forschungsvorhaben auf den Gebieten des Strafrechts, des Strafprozessrechts und der Kriminologie zu realisieren. Im Berichtsjahr 2007 wurden die meisten Forschungsvorhaben zu Einzelfragen des Völkerstrafrechts sowie des Europäischen Strafrechts durchgeführt, aber auch Projekte zu Computer Crime, zur Praxis der Urteilsabsprachen in einzelnen Ländern oder zur
Eine bedeutende Anzahl von Gastwissenschaftlern/innen (37\%) kam im Berichtsjahr 2007 - wie bereits in den vergangenen Jahren - aus Ländern, deren rechtswissenschaftliche Fakultäten traditionell enge Beziehungen zur deutschen Strafrechtswissenschaft und Kriminologie unterhalten (Italien, Spanien, Polen und Türkei). Bemerkenswert ist der Anstieg der Anzahl von Wissenschaftler/innen aus dem Iran (17) und China (10). Aus Finnland hielten sich 9 Wissenschaftler/innen am Institut auf und aus Norwegen und Südkorea waren es jeweils 7. Aus Georgien, Israel und Südafrika kamen jeweils 5 Wissenschaftler/innen. Im Übrigen sind unter den Herkunftsländern Afgha-
Abbildung 1: Anzahl der Gastwissenschaftler/innen am Institut in den Jahren 2005-2007

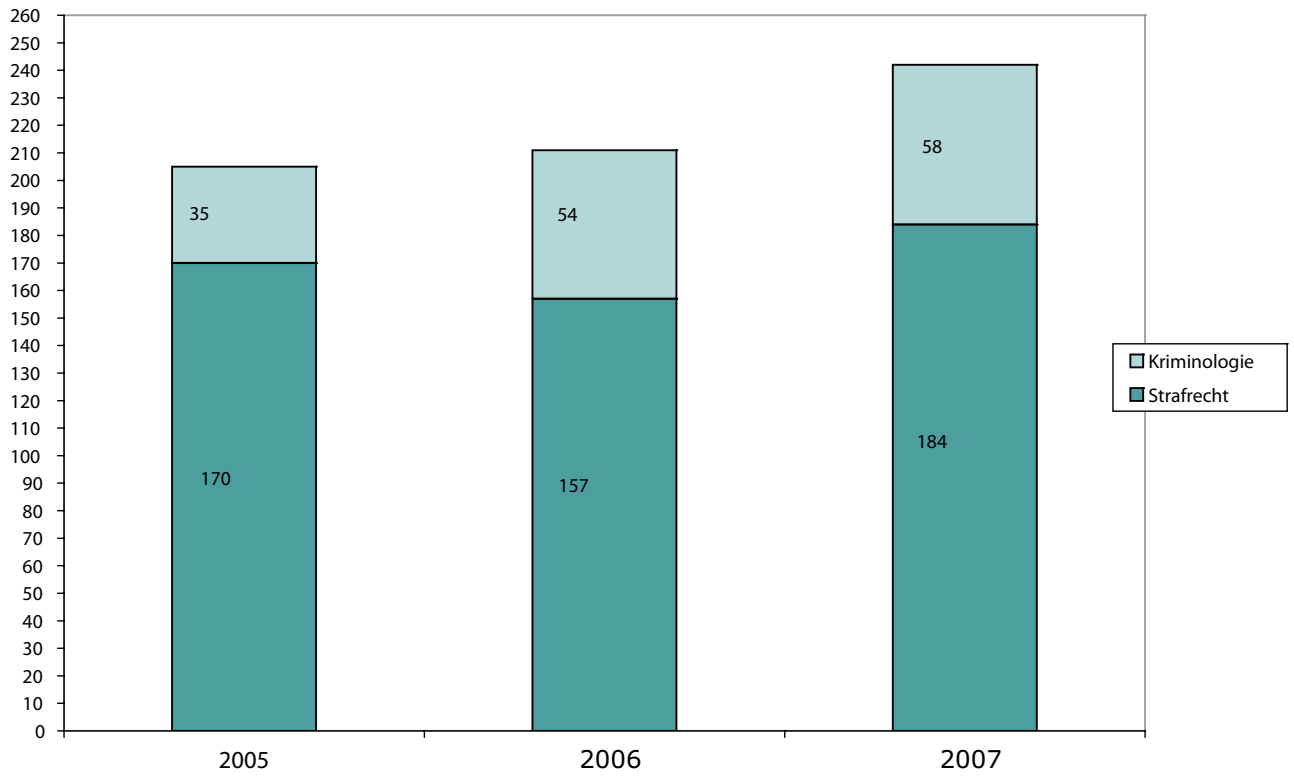

Beschränkung der Grundrechte im Kampf gegen den Terrorismus fanden großes Interesse.

Das Institut wurde im Berichtsjahr 2006 von 211 Gastwissenschaftler/innen aus aller Welt zu Forschungsaufenthalten genutzt, davon kamen $74 \%$ über die strafrechtliche Abteilung und $26 \%$ über die kriminologische Abteilung. Im Berichtsjahr 2007 stieg die Anzahl der Gastwissenschaftler/ innen auf 242 an. Abbildung 1 stellt den Trend der Forschungsaufenthalte in der strafrechtlichen und der kriminologischen Abteilung 2005-2007 dar. nistan, Angola, Argentinien, Australien, Belgien, Bosnien und Herzegowina, Botswana, Brasilien, Bulgarien, Burkina Faso, Chile, Costa Rica, Dänemark, Deutschland, Frankreich, Griechenland, Großbritannien, Indien, Japan, Kamerun, Kanada, Kolumbien, Kongo, Kroatien, Namibia, Niederlande Österreich, Portugal, Russland, Schweden, Schweiz, Serbien, Slowenien, Tschechien, Ukraine, Ungarn, Uruguay und USA repräsentiert. Unter den im Berichtsjahr 2007 anwesenden Wissenschaftler/innen befanden sich 81 Professoren/ innen, 60 Promovierte, 99 Doktoranden und 2 Staatsanwälte. 


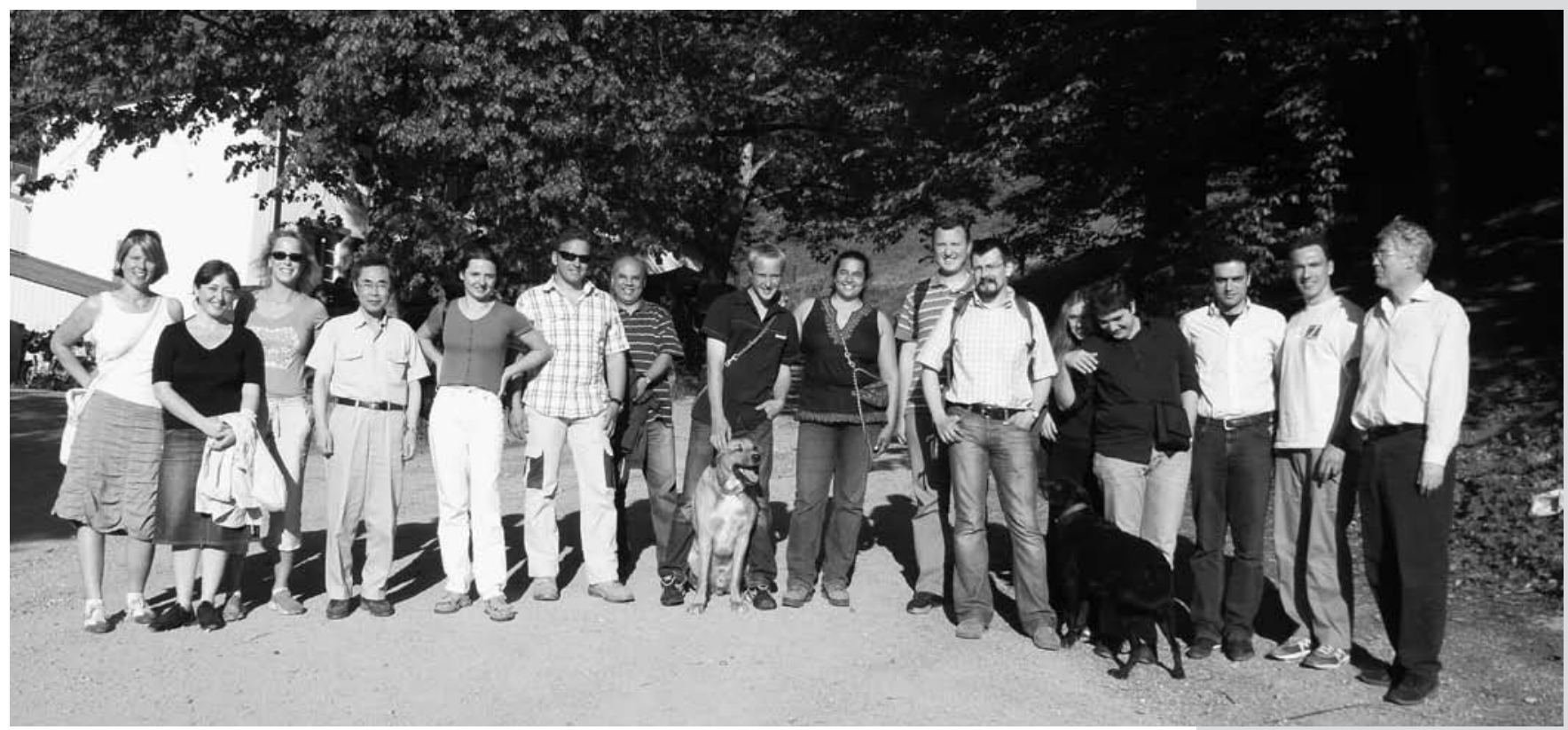

1. Afghanistan (1)

2. Angola (1)

3. Argentinien (3)

4. Australien (2)

5. Belgien (1)

6. Bosnien

und Herzegowina (1)

7. Botswana (1)

8. Brasilien (2)

9. Bulgarien (1)

10. Burkina Faso (1)

11. Chile (2)

12. Costa Rica (3)

13. Dänemark (3)
14. Deutschland (7)
15. Finnland (9)
16. Frankreich (2)
17. Georgien (5)
18. Griechenland (1)
19. Indien (1)
20. Iran (17)
21. Israel (5)
22. Italien (42)
23. Japan (4)
24. Kamerun (1)
25. Kanada (1)

13. Dänemark (3)

15. Finnland (9)

16. Frankreich (2)

17. Georgien (5)

19. Indien (1)

25. Kanada (1)
26. keine Angaben (1)

27. Kolumbien (1)

28. Kongo, Dem. Rep. (1)

29. Kroatien (4)

30. Namibia (1)

31. Niederlande (2)

32. Norwegen (7)

33. Österreich (2)

34. Polen (17)

35. Portugal (1)

36. Russland (4)

37. Schweden (4)

38. Schweiz (1)
39. Serbien (1)

40. Slowenien (2)

41. Spanien (22)

42. Südafrika (5)

43. Südkorea (7)

44. Tschechien (4)

45. Türkei (9)

46. UK (4)

47. Ukraine (1)

48. Ungarn (7)

49. Uruguay (1)

50. USA (6)

51. V.R. China (10)

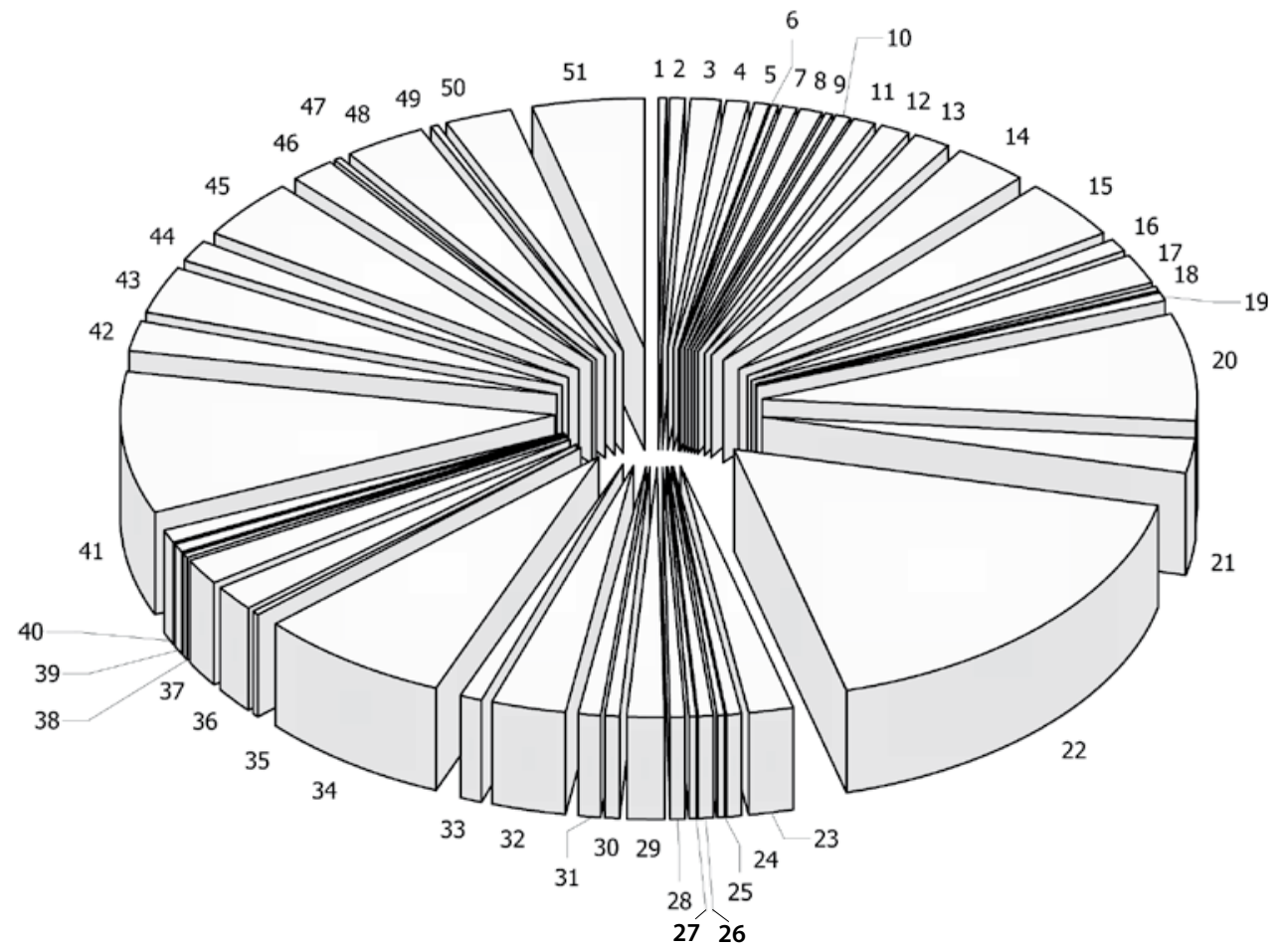

Abbildung 2:

Verteilung der ausländischen

Wissenschaftler/innen auf die

Herkunftsländer 2007

Anzahl der Gäste insgesamt: 242 


\title{
C. Gutachten
}

\author{
Zusammenarbeit von Wissenschaft und Praxis
}

Aus der Verbindung praktischer Fragestellungen mit denen des Forschungsprogramms der strafrechtlichen Abteilung ergeben sich wertvolle Anregungen für die Forschungsarbeit. Einzelne Anfragen aus der Praxis von Rechtspflege, Politik oder Verwaltung führen zu rechtsvergleichenden Großprojekten, deren Bearbeitung nicht ohne die Mitarbeit externer Wissenschaftler aus dem Ausland möglich ist. Im Berichtszeitraum trifft dies insbesondere auf eine Anfrage des Bundesverfassungsgerichts zur Strafbarkeit des Geschwister-Inzests zu, aus der - thematisch über den ursprünglichen Gutachtenauftrag hinausgehend - ein rechtsvergleichend-kriminologisches Gesamtprojekt entwickelt wurde (siehe dazu oben II.J.). Erhebliche Bedeutung für die zukünftige Forschungsarbeit des Instituts haben auch die Ergebnisse eines Gutachtens für das Bundesverfassungsgericht über die Online-Durchsuchung.

Das Max-Planck-Institut ist grundsätzlich bereit, Justizorgane und Behörden in Fragen der Strafbar- keit nach ausländischem Recht bei Auslandsbezügen von Tat und/oder Täter, sowie bei Fragen der Zusammenarbeit im Wege der Rechtshilfe, der Auslieferung oder bezüglich ausländischer Strafverfahrensrechte, zu beraten. Dies korrespondiert mit der gegenüber diesen Institutionen bestehenden Erwartung des Max-Planck-Instituts, insbesondere bei empirischen Forschungsprojekten die nötige Unterstützung zu erfahren.

Im Berichtszeitraum wurden insgesamt 162 Anfragen bearbeitet (2006: 93, 2007:69), davon 110 von Institutionen der Rechtspflege (2006: 63; 2007: 47). Der Rückgang gegenüber früheren Jahren ist zum einen Folge der geringeren Anzahl von Asylverfahren, die bislang einen gewissen Schwerpunkt der Anfragen darstellten. Zum anderen ergibt er sich aus der Übertragung von Medienanfragen an das Referat „Öffentlichkeitsarbeit“. Zu den am meisten nachgefragten Ländern zählen neben Deutschland (43) Spanien (12), Polen (9), die Türkei (9) und die USA (7). 


\section{Veranstaltungen und Vorträge}

\section{Veranstaltungen des Instituts}

Im Berichtszeitraum 2006/07 fand wie in den Vorjahren eine Vielzahl von wissenschaftlichen Veranstaltungen des Max-Planck-Instituts statt, die im Einzelnen im Anhang unter $C$ aufgeführt sind. Es handelt sich dabei um Veranstaltungen $i m$ Institut sowie externe Veranstaltungen des Instituts. Die Veranstaltungen verteilten sich einerseits auf die beiden wissenschaftlichen Abteilungen, sie wurden andererseits aber auch gemeinsam organisiert und durchgeführt. Gemeinsame Veranstaltungen waren überwiegend Tagungen und Kolloquien, die im Institut stattfanden. Eine herausragende Stellung kam dabei dem gemeinsam mit der Albert-Ludwigs-Universität Freiburg veranstalteten Kolloquium zum 40-jährigen Bestehen des Max-Planck-Instituts am 21.07.2006 zu, in dessen Mittelpunkt eine interdisziplinäre Diskussion über „Aufgaben, Methoden und Perspektiven der Rechtsvergleichung im Zivilrecht, öffentlichen Recht, Strafrecht, Kriminologie, Arbeitsrecht und Prozessrecht" stand. Daran beteiligten sich nicht nur die Auswärtigen Wissenschaftlichen Mitglieder des Max-Planck-Instituts von der Universität Freiburg (Prof. Dr. Wolfgang Frisch und Prof. Dr. Walter Perron), sondern auch eine Reihe weiterer Lehrstuhlinhaber der genannten Rechtsfächer an der Universität.

Bei den Tagungen und Kolloquien spielen Besuche am Max-Planck-Institut, insbesondere durch ausländische Praktikerdelegationen, eine immer gröBere Rolle. Diese Besuche werden verbunden mit

\section{Vorträge aus dem Institut}

Die Vortragstätigkeit der Direktoren und der wissenschaftlichen Mitarbeiter dient verschiedenen Zielen. Zum einen werden durch Vorträge die Ergebnisse der Forschung anlässlich nationaler oder internationaler Kongresse und Workshops vorgestellt. Die Teilnahme an Vortragsveranstaltungen und Reisen zu Universitäten oder Forschungseinrichtungen des In- und Auslands sind auch auf die Vorbereitung und Intensivierung der einem intensiven gegenseitigen Erfahrungsaustausch zwischen den Wissenschaftlern des Instituts und den Juristen aus den jeweiligen Ländern.

Der wissenschaftlichen Diskussion zwischen den Mitarbeitern des Instituts vor allem mit den ausländischen Langzeitgästen dienen die Vortragsveranstaltungen in der strafrechtlichen sowie in der kriminologischen Abteilung. Dabei haben sich die grundlagenorientierten sogenannten „Mittwochsvorträge“ der strafrechtlichen Abteilung ebenso etabliert wie die mehr unter aktuelleren Gesichtspunkten veranstalteten Vorträge der kriminologischen Abteilung.

Die Doktoranden des Max-Planck-Instituts erhalten Gelegenheit, ihre Dissertationsvorhaben und bereits erzielten Ergebnisse in den Vortragsreihen der beiden Abteilungen vorzustellen. Sie nutzen zudem die Gelegenheit, ihre Arbeiten in den regelmäßig stattfindenden Doktorandentreffen, die institutsoffen sind, zu präsentieren und sich dabei der kritischen Diskussion zu stellen. Vor diesem Hintergrund sind auch in den Vortragsveranstaltungen Foren der Integration und Förderung des wissenschaftlichen Nachwuchses des MaxPlanck-Instituts zu sehen.

Unter den externen Veranstaltungen des Instituts sind besonders bilaterale wissenschaftliche Treffen mit einigen lateinamerikanischen Ländern hervorzuheben (Argentinien und Brasilien).

wissenschaftlichen Kooperationen gerichtet. Zum anderen zielt die Vortragstätigkeit auf die Fortund Ausbildung an in- und ausländischen Akademien (beispielsweise Deutsche Richterakademie) und auf solche Berufsgruppen, die im System der Strafverfolgung, der Strafjustiz und des Strafvollzugs tätig sind. Die Vortragstätigkeit schließt auch Veranstaltungen ein, die sich an die Politik und die Öffentlichkeit wenden. 
Im Berichtszeitraum wurden - wie sich näher aus dem Anhang des vorliegenden Forschungsberichts unter $B$ ergibt - insgesamt 366 Vorträge gehalten, davon $221 \mathrm{im}$ Inland und $145 \mathrm{im}$ Ausland. Die Auslandsvorträge verteilten sich auf 37 Länder, die meisten in Italien (15), gefolgt von China, Iran und Ungarn mit jeweils 14 Vorträgen. Von den Inlands- vorträgen fanden 88 in Freiburg statt. Strukturell lassen sich die Vorträge untergliedern in Beiträge auf Kongressen, Workshops und Kolloquien (122), am Institut (82), an ausländischen Universitäten (45), an Akademien (25), Schulen (24), vor Verbänden (19) und für die Öffentlichkeit (19), die Politik (17) sowie vor Studienstiftungen (9). 


\section{E. Lehre}

\section{LEHRE IM INLAND}

\author{
a) Lehre an der Universität
}

Lehraktivitäten an der Universität erfolgen durch die Direktoren Prof. Dr. Ulrich Sieber und Prof. Dr. Hans-Jörg Albrecht sowie durch die wissenschaftlichen Mitarbeiter Prof. Dr. Jörg Arnold, Martin Brandenstein, Marc Engelhart, Dr. Michael Kilchling, Priv.-Doz. Dr. Hans-Georg Koch, Dr. Tilman Köllisch, Priv.-Doz. Dr. Dietrich Oberwittler, Figen Özsöz, Dr. Johanna Rinceanu (vgl. Anhang unter C). Diese Aktivitäten belegen eine weitere wichtige Form der Zusammenarbeit des

\section{b) Elektronische Lehre (E-Learning)}

Neben den oben erwähnten Präsenz-Lehrveranstaltungen im In- und Ausland ist das Institut auch im Bereich E-Learning, d.h. der elektronischen Lehre über das Internet, aktiv. Das Pilotprojekt „Cueno“ (http://www.cueno.de) wurde ursprünglich an der Ludwig-Maximilians-Universität begonnen und am Max-Planck-Institut weiterentwickelt und ausgebaut.

Im Berichtszeitraum wurden die dogmatischen Ausführungen und die Fallbeispiele zusätzlich

\section{LEHRE IM AUSLAND}

Die Lehrtätigkeit des Max-Planck-Instituts ist nicht auf nationale Universitäten beschränkt, sondern betrifft in vielfältiger Weise auch Kooperationen mit Wissenschaftsinstitutionen im Ausland. Vielfach dient das Engagement wie beispielsweise der Rechtsstaatsdialog mit China der „Entwicklung ausländischer Rechtssysteme“. Dabei handelt es sich jedoch nicht etwa um die einseitige Zielstellung des Exports der deutschen Lehre in das Ausland. Vielmehr profitiert das Max-Planck-
Max-Planck-Instituts mit Universitäten, insbesondere mit der Albert-Ludwigs-Universität Freiburg. Die Lehrenden des Max-Planck-Instituts wirken nicht nur aktiv daran mit, den Lehrbedarf der Universitäten in den Fächern Strafrecht und Kriminologie zu decken, sondern leisten inhaltlich darüber hinaus einen wichtigen Beitrag dazu, den Studierenden die Forschungen des Max-PlanckInstituts und dabei auch internationale wie interdisziplinäre Aspekte nahe zu bringen.

durch kriminologische Texte zur Lektion „Mord und Totschlag" ergänzt. Diese Texte wurden ebenfalls zum Abruf zur Verfügung gestellt. Im Jahr 2006 war damit die Entwicklungsarbeit am Pilotprojekt abgeschlossen. Trotz der überaus positiven Evaluation ist kein weiterer Ausbau vorgesehen, da die Ressourcen des Instituts auf Fragen der Grundlagenforschung konzentriert werden sollen. Das Angebot in der gegenwärtigen Form, das nach wie vor regen Zuspruch erfährt, soll jedoch für einige Zeit weiter aufrecht erhalten werden.

Institut für seine eigenen Forschungen: Die Kenntnisse und Erfahrungen, die von den Lehrenden über die jeweilige ausländische Rechtsordnung gewonnen werden, kommen der Rechtsvergleichung unmittelbar zugute. Diese Aspekte der Lehre werden nachfolgend näher dargestellt. Weitere Lehrtätigkeiten von Mitarbeitern des Max-Planck-Instituts an ausländischen Universitäten sind im Anhang unter $C$ aufgeführt (Adome Blaisse Kouassi, Marianne Wade).
Prof. Dr. Ulrich Sieber, Phillip W. Brunst 
Prof. Dr. Ulrich Sieber, Marc Engelhart, Julia Macke, Yang Zhou
Nähere Informationen zum Projekt finden sich unter: http://www. mpg.de/bilderBerichteDokumente/ dokumentation/jahrbuch/2007/ voelkerrecht/forschungsSchwerpunkt/index.html

\section{a) Richterausbildung in China}

Zur Ausbildung junger Richter, aber auch von Richterausbildern selbst, hat der Legal Advisory Service der GTZ (Deutsche Gesellschaft für technische Zusammenarbeit, Büro in Peking) zusammen mit der Nationalen Richterakademie (National Judges College) in Peking das Projekt zur „Chinesisch-Deutschen Zusammenarbeit zur Richterausbildung" initiiert. Teil des Programms ist die Durchführung eines Kurses im Strafrecht. Mit der Erstellung der Kursunterlagen und der inhaltlichen Begleitung des ersten Kurses im Strafrecht wurde das Freiburger Max-Planck-Institut beauftragt.

Ziel des Projekts ist es, eine methodische und rechtsstaatliche Bearbeitung strafrechtlicher Fälle durch chinesische Richter zu erreichen. Die Richter sollen in die Lage versetzt werden, mithilfe der Rechtsmethodik eine einheitliche und an rechtsstaatlichen Prinzipien orientierte Strafrechtsanwendung im richterlichen Alltag vornehmen zu können. Dazu wurden geeignete Lehrmaterialien erstellt, die im Bereich des Allgemeinen Teils des chinesischen Strafrechts eine dogmatisch fundierte, methodisch klare und rechtsstaatlich gesicherte Rechtsanwendung vermitteln.

Für die Konzeption des Kurses wurde ein fallbasierter Ansatz gewählt, der den Fallkonstellationen des richterlichen Alltags entspricht. Die
Kursunterlagen wurden auf Englisch und Chinesisch erstellt. Aus dem Bereich des chinesischen Strafrechts Allgemeiner Teil wurden zwanzig exemplarische Problembereiche ausgewählt, die sowohl in dogmatischer wie auch in methodischer Hinsicht eine Veranschaulichung grundlegender Prinzipien und Funktionen des rechtsstaatlichen Strafrechts erlaubten. Rechtsvergleichende Hinweise zum deutschen Recht verdeutlichten die Funktionsweise und Bedeutung leitender Prinzipien. Ergänzend wurden zehn Trainingseinheiten mit kürzeren Fallkonstellationen zur Vertiefung und Veranschaulichung bestimmter Fragenkomplexe des Allgemeinen Teils entworfen. Bei der Erstellung der Materialien kooperierten chinesische und deutsche Institutsmitarbeiter.

Die erstmalige Durchführung des Kurses erfolgte Ende 2006 in Peking. Der zweiwöchige Kurs in englischer Sprache mit chinesischer Übersetzung wurde durch einen Institutsmitarbeiter (Marc Engelhart) geleitet und durch einen weiteren Mitarbeiter (Yang Zhao) begleitet. Die Vermittlung eines methodischen Vorgehens unter Berücksichtigung rechtsstaatlicher Grundsätze stieß bei den Teilnehmenden des Kurses (60 Richterinnen und Richter) auf großes Interesse, da sich bei den meisten Gerichten noch keine prinzipiengeleitete Arbeitspraxis entwickelt hat.

\section{b) Förderung des Reformprozesses in Afghanistan}

Zur Stabilisierung des Justizwesens in Afghanistan organisiert das Max-Planck-Institut für ausländisches öffentliches Recht und Völkerrecht in Heidelberg seit 2003 mehrere Projekte. Eines dieser Projekte unterstützt in Zusammenarbeit mit dem Obersten Gericht Afghanistans die Ausbildung der Richteranwärter in Afghanistan. Der strafrechtliche Teil dieses Projekts wird vom Max-Planck-Institut für ausländisches und internationales Strafrecht betreut.

Für den Unterricht erstellte Mohammad Sadr Touhid-Khaneh, Mitarbeiter des Freiburger Instituts, das „Max-Planck-Manual über den Allgemeinen Teil des afghanischen Strafrechts" (Heidelberg/Kabul 2007) sowie zusätzliche Materialien auf Dari. Das Kurzlehrbuch behandelt zentrale Fragen der Strafrechtsdogmatik in Afghanistan. Die eingehende Analyse bezieht sich nicht nur auf die Quellen der Scharia und der
Strafgesetze, sondern berücksichtigt auch die verfassungsrechtlichen Vorgaben.

Im Rahmen dieses Projekts bereitete der Mitarbeiter auch die Dozenten des Projekts „Strafrechtliche Ausbildung von Richteranwärterinnen und Richteranwärtern in Afghanistan" in einem Workshop am Freiburger Institut auf ihre Lehrtätigkeit in Kabul vor und betreute ihren Unterricht vor Ort an der Universität Kabul wissenschaftlich. Als Dozent einer Fortbildung für Richter und Staatsanwälte in den vom Heidelberger Max-Planck-Institut veranstalteten Workshops hielt er in Kabul den Unterricht eines weiteren Moduls über „Prinzipien eines fairen Strafverfahrens in Afghanistan“.

Das von Sadr Touhid-Khaneh erstellte Kurzlehrbuch wird im Jahr 2008 aktualisiert. Der Ausbildungskurs wird auch in diesem Jahr vor Ort fortgesetzt. 
c) Sommerkurs in Pécs, Ungarn

Seit dem Jahr 2006 führt das Max-Planck-Institut für ausländisches und internationales Strafrecht regelmäßig einmal im Jahr einen Sommerkurs in Zusammenarbeit mit der Universität Pécs in Ungarn durch. Die Kurse werden in Ungarn abgehalten und finden in deutscher Sprache statt. Sie richten sich an Studierende, Staatsanwälte, Richter und andere Interessierte, die sich über aktuelle Forschungsfragen des Instituts informieren möchten. Die Organisation wird abwechselnd von der kriminologischen und der strafrechtlichen Abteilung übernommen; als Vortragende kommen etablierte Forscher und wissenschaftlicher Nachwuchs gleichermaßen zum Einsatz.

Die erste Sommerschule, die 2006 unter Leitung der kriminologischen Abteilung durchgeführt wurde, stand unter dem Thema „Neuere Entwicklungen in der kriminologischen Theorie und kriminologischen Forschung“. Es wurden sowohl theoretische als auch empirische Fragestellungen, mit denen sich die kriminologische Abteilung gegenwärtig befasst, präsentiert und diskutiert.
Dabei liegt der Schwerpunkt auf den Ergebnissen der Institutsprojekte, wie z.B. der Freiburger Kohortenstudie. Jeder Tag stand unter einer anderen Thematik, die zunächst jeweils im Rahmen von Präsentationen vorgestellt und anschließend in Arbeitsgruppen intensiv bearbeitet wurde. Am Ende jeden Tages wurden die Ergebnisse in einer Diskussionsrunde im Plenum abschließend bewertet.

Die nächste Sommerschule im Jahr 2007 wurde von der strafrechtlichen Abteilung organisiert. Ihr Thema war „Aktuelle Kriminalitätsentwicklung, Internationales Strafrecht und Strafrechtsvergleichung - Forschungsfragen an den Grenzen des Strafrechts“. Im Mittelpunkt standen die durch das Forschungsprogramm der strafrechtlichen Abteilung aufgeworfenen neuen Herausforderungen des Strafrechts, insbesondere transnationale und komplexe Kriminalität, wie z.B. Internet-Kriminalität, organisierte Kriminalität und Terrorismus. Die einschlägigen rechtlichen Fragen wurden rechtsvergleichend behandelt und mit Einführungen in
Prof. Dr. Ulrich Sieber,

Prof. Dr. Hans-Jörg Albrecht, Mitarbeiter der strafrechtlichen und der kriminologischen Abteilung
Trainer und Schüler der Strafrechtlichen Richterausbildung in der Universität Kabul

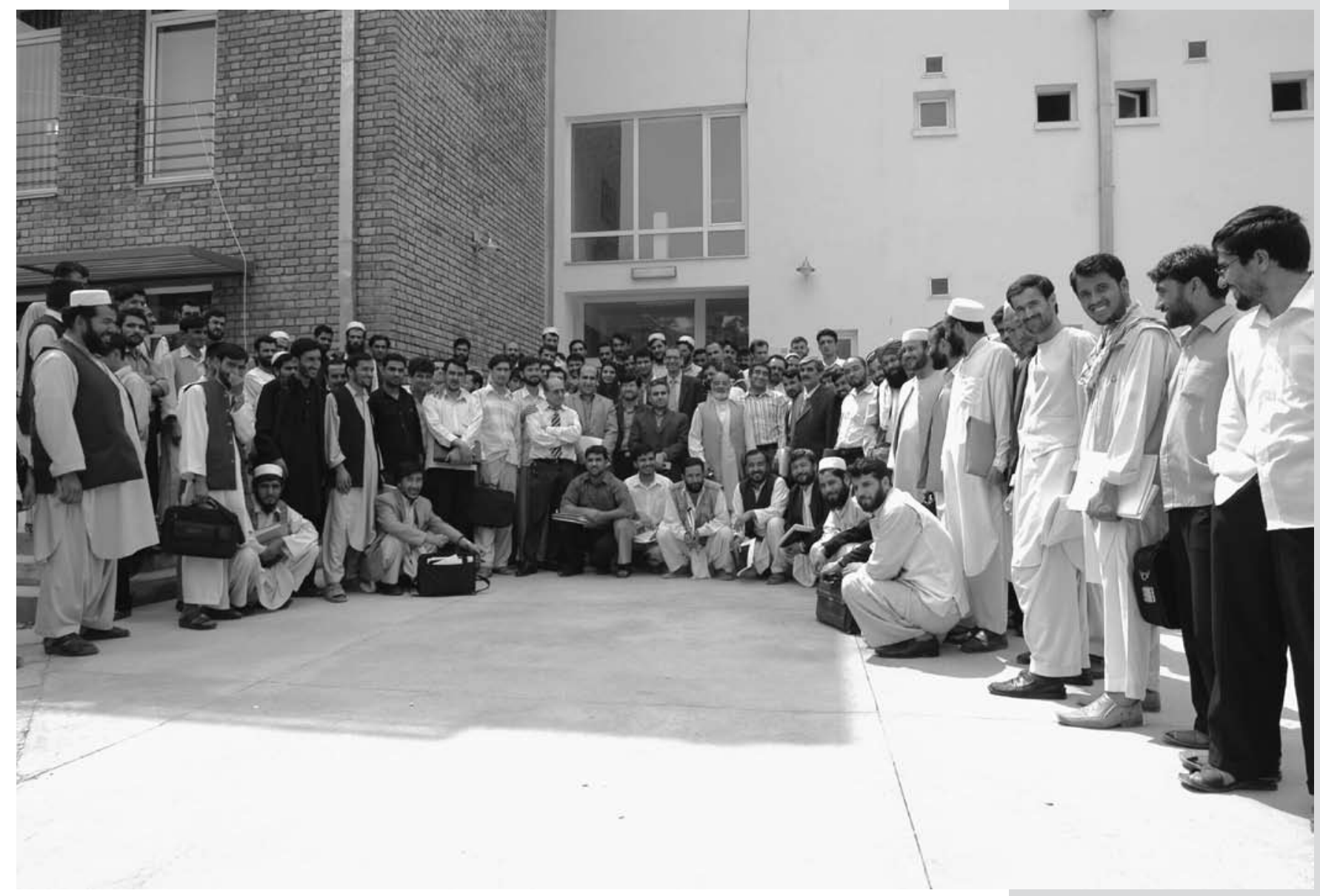




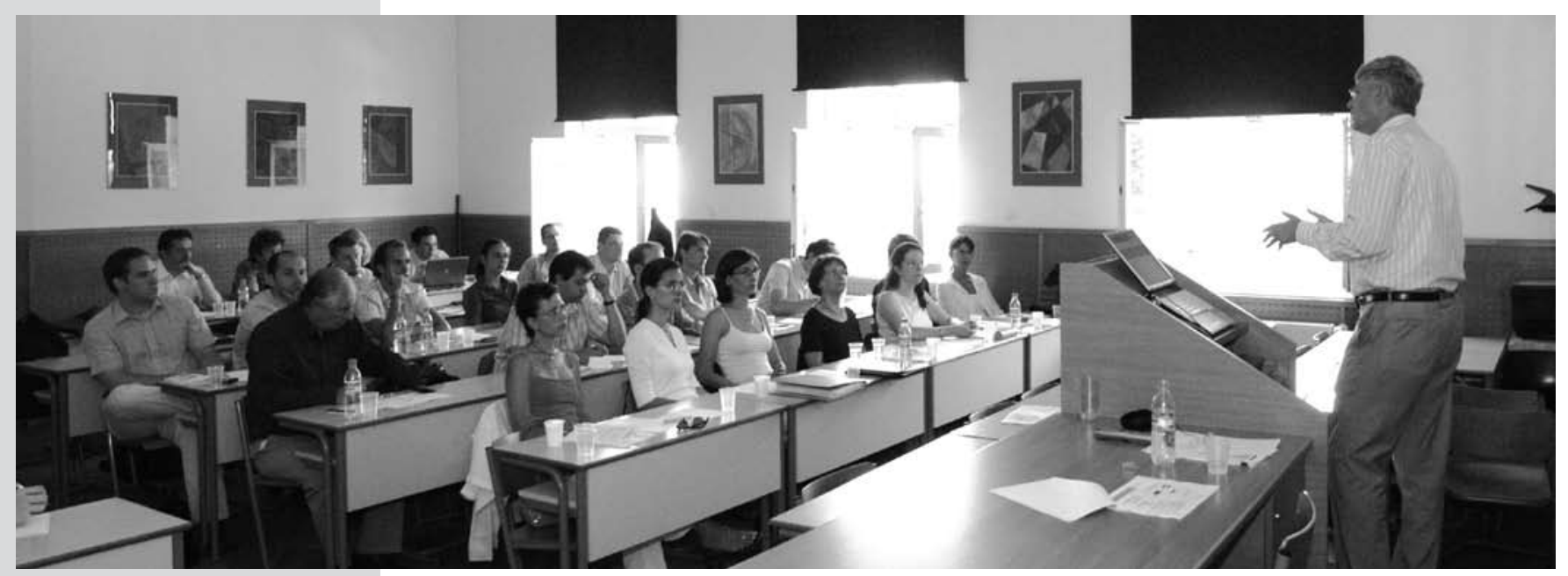

Einführungsveranstaltung der Sommerschule 2007 in Pécs ausländische Rechtsordnungen verbunden. Die zunehmende Internationalisierung des Strafrechts wurde zudem auch am Europäischen und am internationalen Strafrecht deutlich gemacht.

Die Veranstaltungen werden sehr gut angenommen und fördern eine langjährige europäische Zusammenarbeit zwischen Strafrechtlern und Kriminologen verschiedener Nationen. Dies zeigt sich nicht zuletzt an der Tatsache, dass die letzte Veranstaltung im Jahr 2008, die wieder von der kriminologischen Abteilung durchgeführt wurde und unter dem Motto „Technik, Überwachung, Strafverfahren" stand, von Teilnehmern aus insgesamt 10 Nationen besucht wurde.

Für das Jahr 2009 ist wiederum eine Veranstaltung der strafrechtlichen Abteilung geplant. 
V. Organisation 


\section{ORGANISATION}

A. Forschungsabteilungen

B. Bibliothek

C. Publikationsabteilung

D. Öffentlichkeitsarbeit

E. EDV-Dienstleistungen

F. Forschungsförderung

G. Fachbeirat und Kuratorium 


\section{A. Forschungsabteilungen}

\section{STRAFRECHTLICHE ABTEILUNG}

Die Zusammensetzung und die Organisation der strafrechtlichen Abteilung werden durch ihr Forschungsprogramm bestimmt: Die Arbeit am Institut soll nicht aus der Addition isolierter Einzelprojekte bestehen, sondern durch die Bündelung von Kapazitäten Synergieeffekte für die wissenschaftliche Erkenntnis und Ausbildung erzielen. Vor allem bei größeren Projekten sind oft mehrere wissenschaftliche Mitarbeiter der Abteilung mit ihrem Fachwissen in unterschiedlichen Projektphasen eingebunden. Dieses Fachwissen bildet das wissenschaftliche Rückgrat der strafrechtsvergleichenden Forschung. Daneben liegen Fragen der Ausbildung des wissenschaftlichen Nachwuchses sowie Gutachten-, Übersetzungs- und Publikationsfragen in der Hand von Fachleuten, die für die Forschungsunterstützung zuständig sind.

\section{Forschung}

Die Forschung in der strafrechtlichen Abteilung erfordert zunächst ein nach Ländern und Sachgebieten ausdifferenziertes Fachwissen. Ein Forschungsprogramm mit der gegenständlichen und der methodischen Ausrichtung auf die Strafrechtsvergleichung verlangt insbesondere ein detailliertes Wissen über das ausländische sowie das europäische und internationale Strafrecht, über die verschiedenen Rechtskulturen und die sie tragenden Gesellschaften sowie die dafür erforderlichen Fremdsprachenkenntnisse auf wissenschaftlichem Niveau. Diese besonderen Qualifikationen sind schwer zu finden. Denn das Strafrecht ist wie kaum eine andere Rechtsmaterie Ausdruck der staatlichen Souveränität, noch wenig internationalisiert und daher eine Materie von Spezialisten für im Einzelnen sehr unterschiedliche Rechtsordnungen. Ähnliches gilt für Sachgebiete, die durch ihre besondere Komplexität in tatsächlicher und in rechtlicher Hinsicht gekennzeichnet sind und häufig über strafrechtliche Aspekte hinausreichen. Auch hier sind die Anforderungsprofile so speziell, dass Experten selten sind. Die Einbeziehung von Fachwissen kann auch nur begrenzt über die - in jedem Fall notwendige - Zusammenarbeit mit externen Wissenschaftlern gelöst werden. Die erforderlichen Kernkompetenzen müssen deswegen in bestimmten Sachbereichen von der Forschungsabteilung selbst bereitgehalten werden, wenn diese im internationalen Wettbewerb bestehen will.

\section{Länderspezifisches Fachwissen}

Die Zusammensetzung des Kreises der wissenschaftlichen Mitarbeiter trägt der Internationalität Rechnung. Die Anzahl der ausländischen Mitarbeiter nimmt deswegen auch zu. Das dadurch einbezogene Fachwissen umfasst einzelne Länder (z.B. Italien oder Spanien), verwandte Rechtsordnungen (z.B. Nordische Länder), Regionen (z.B. Afrika südlich der Sahara) und Sub-Kontinente (z.B. Lateinamerika). Darüber hinaus sind Rechtsordnungen des Common Law (USA und Kanada sowie das Vereinigte Königreich) und von muslimisch geprägten Gesellschaften (Türkei, Iran, arabische Länder) einbezogen. Zu den betreffenden Rechtsordnungen werden laufend die aktuelle Entwicklung der Gesetzgebung, Rechtsprechung und Lehre sowie die Tendenzen der Kriminalität und der Kriminalpolitik verfolgt.

Für die wissenschaftlichen Mitarbeiter ist die Mitwirkung an rechtsvergleichenden Gemeinschaftsprojekten der Forschungsabteilung von besonderer Bedeutung. Hier kommen ihre speziellen Kenntnisse über die fremden Rechtsordnungen bereits bei der Forschungskonzeption zum Tragen. Der gegenseitige Meinungs-, Gedanken- und Erfahrungsaustausch über das Rechtsverständnis in den einzelnen Ländern ermöglicht einen übergreifenden Blick auf den Untersuchungsgegenstand und die von länderspezifischen Begriffen 
losgelöste Formulierung von Forschungsfragen. Bei größeren rechtsvergleichenden Arbeiten wird vom Institutsdirektor und den Projektleitern eine Gliederung entwickelt, die den Landesberichten als Basis für die Erstellung des anschließenden Rechtsvergleichs zugrunde liegt. Dieses Vorgehen ist Bedingung für eine fundierte rechtsvergleichende Grundlagenforschung.

Neben den Gemeinschaftsprojekten sind die wissenschaftlichen Mitarbeiter auch in Einzelprojekten tätig. Sie informieren die Fachöffentlichkeit in Aufsätzen und Vorträgen - auch rechtsvergleichend - über Entwicklungen und Besonderheiten des Strafrechts und der Kriminalpolitik in den einzelnen Ländern. Auf diese Weise tragen die Forscher unter auslandsrechtlichen und rechtsvergleichenden Aspekten zur wissenschaftlichen Diskussion bei. Ferner erhält das Institut häufig Anfragen zu bestimmten Rechtsproblemen oder Verfahrensregeln in einzelnen Ländern. Die meisten Anfragen kommen von deutschen Gerichten und Staatsanwaltschaften aus Anlass konkreter Strafsachen, in denen das ausländische Strafrecht oder die Prozesspraxis eine Rolle spielen. Im Berichtszeitraum wurden mehrere Anfragen des Bundesverfassungsgerichts zu wichtigen Grundlagenfragen bearbeitet. Auch andere deutsche und ausländische Institutionen, insbesondere Justizministerien, aber auch einzelne Personen aus Wissenschaft und Praxis, sind auf entsprechende Informationen angewiesen. Solche Anfragen werden nach Möglichkeit durch Gutachten beantwortet. Darüber hinaus ist die Expertise der Wissenschaftler in Reformprozessen weltweit gefragt.

Die Forschung stützt sich hauptsächlich auf die Institutsbibliothek. Zu den Aufgaben der wissenschaftlichen Mitarbeiter der Abteilung gehört es daher, den Bibliotheksbestand auf dem aktuellen Stand zu halten. Dies setzt eine sorgfältige Beobachtung des Fachbuch- und Zeitschriftenmarktes sowie der elektronischen Medien voraus. Von groBer Bedeutung sind weiter persönliche Kontakte mit Personen aus Wissenschaft und Praxis der jeweiligen Länder. Hierdurch erschließen sich nicht nur zusätzliche Informationsquellen, sondern der Aufbau und die Pflege dieser Beziehungen bilden auch die Basis für die wissenschaftliche Zusammenarbeit des Instituts mit dem Ausland. Die wissenschaftlichen Mitarbeiter der Abteilung stehen in Verbindung mit Universitäten, Strafverfolgungs- und Justizbehörden sowie anderen juristischen Einrichtungen in ihren Ländern. Sie nehmen an Seminaren und Kongressen teil, halten Vorträge, organisieren mit den Partnern der Ab- teilung vor Ort gemeinsame Veranstaltungen und wirken in Einzelfällen an akademischen Prüfungen ausländischer Universitäten mit. Zur Kontaktpflege gehört vor allem auch die Betreuung ausländischer Wissenschaftler, die sich als Forschungsgäste am Institut aufhalten. Hier ist insbesondere die fachliche und persönliche Unterstützung des wissenschaftlichen Nachwuchses hervorzuheben. Aus ihr entstehen die Auslandsbeziehungen zur nachfolgenden Generation von Juristen.

\section{Sachgebietsspezifische wissenschaftliche Expertise}

Neben dem länderspezifischen Sachverstand konzentriert sich die Zusammensetzung der wissenschaftlichen Mitarbeiter in der Abteilung gegenwärtig auf die Expertise zu vier Querschnittsmaterien der Forschung, die unterschiedliche Sachgebiete betreffen: Beim internationalen Strafrecht (insbesondere Völkerstrafrecht) sowie beim Europäischen Strafrecht geht es vor allem um die Untersuchung eigenständiger supranationaler Strafrechtsordnungen, während es im Informationsrecht und im Medizinrecht um besondere Formen der Delinquenz sowie die Möglichkeiten und Grenzen ihrer Kontrolle durch das Strafrecht geht. Wegen der für sie typischen ineinandergreifenden Rechtsgebiete und der Komplexität der technischen und medizinischen Materien ist hier ein besonderes Fachwissen erforderlich. Darüber hinaus sind alle vier Sachgebiete durch spezifische politische bzw. technische Entwicklungsbedingungen gekennzeichnet, die - vergleichbar den charakteristischen Bedingungen einer nationalen Strafrechtsordnung - der besonderen Beobachtung und Erforschung bedürfen. Aufgaben und Arbeitsweisen der Mitarbeiter mit besonderem Fachwissen ähneln dabei denen der Mitarbeiter mit länderspezifischem Wissen.

International Max Planck Research School for Comparative Criminal Law (IMPRS-CC)

Neben den Forschungsarbeiten der Mitarbeiter aus der strafrechtlichen Abteilung ist auch das Ausbildungsprogramm der „International Max Planck Research School for Comparative Criminal Law" (IMPRS-CC) in die Forschungsfelder des Forschungsprogramms eingebettet (vgl. Kapitel I.B. 1 „Forschungsprogramm“ und III.C „IMPRSCC). Auf diese Weise trägt auch der wissenschaftliche Nachwuchs der IMPRS-CC seinen Teil zum Forschungsbetrieb der Abteilung bei. 
Die Forschungsarbeit wird durch Fachleute unterstützt und ergänzt, in deren Hand die Publikation und Übersetzung, die Koordination der gutachterlichen Tätigkeiten und der International Max Planck Research School for Comparative Criminal Law liegen.

Die Unterstützung bei der Publikation betrifft vor allem die Forschungsergebnisse. Hierbei spielt insbesondere bei Untersuchungen, an denen eine größere Anzahl von externen Autoren beteiligt ist - die sprachliche und redaktionelle Arbeit eine besondere Rolle. Diese Arbeit wird von Publikationsfachleuten übernommen.

Deutsch ist im Strafrecht eine immer noch bedeutende Wissenschaftssprache. Aus diesem Grund und auch wegen der historisch gewachsenen Struktur der Forschungsabteilung wurden die Forschungsergebnisse bislang vor allem in deutscher Sprache veröffentlicht. Zur Unterstützung der beabsichtigten stärkeren Publikation in englischer Sprache bedarf es entsprechender Fachmitarbeiter, insbesondere für das „Proofreading" und für Übersetzungen. Fremdsprachliche Publikationen nehmen in der Abteilung ebenso kontinuierlich zu wie die Zahl ihrer ausländischen wissenschaftlichen Mitarbeiter.

Angesichts der zahlreichen Rechtsfragen, die von außen an die Forschungsabteilung gestellt werden, besteht auch ein spezieller Bedarf zur Unterstützung der gutachterlichen Tätigkeiten, insbesondere im Hinblick auf die Koordination des Gesamtablaufs der Gutachtenbearbeitung, die Beratung der Fragesteller, die Prüfung der Anfragen auf ihre Bezüge zum Forschungsprogramm, die Weiterleitung der relevanten Fragen an die Wissenschaftler und die Zusammenfassung von Einzelergebnissen.

Schließlich bedarf der Forschungs- und Ausbildungsbetrieb der International Max Planck Research School for Comparative Criminal Law

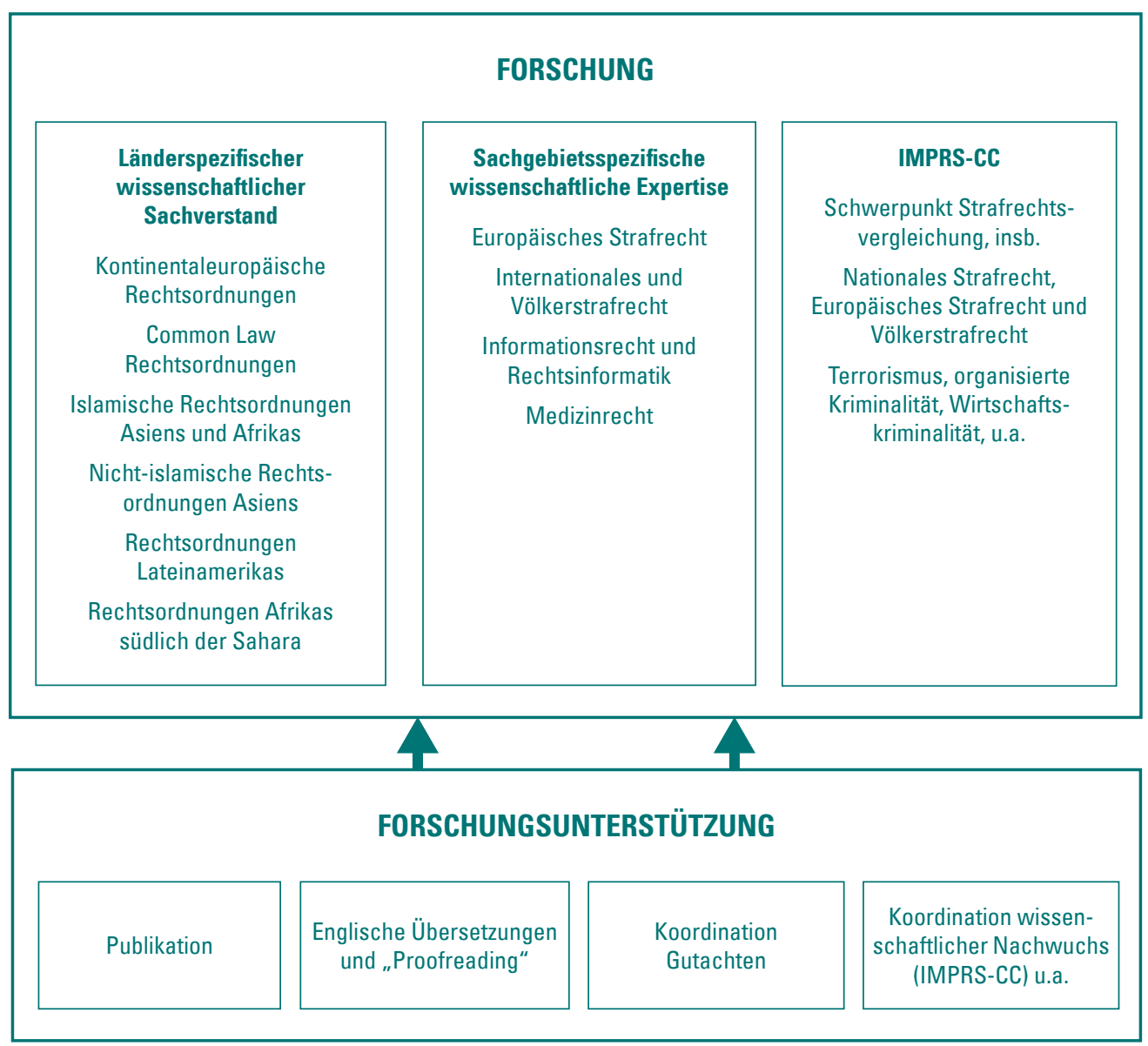

Schema:

Spezialisiertes Fachwissen in der strafrechtlichen Forschungsabteilung 
der Koordination. Dabei geht es vor allem um die Gesamtkoordination der Ausbildungsmaßnahmen nach den Vorgaben des Lenkungsausschusses und um die Beratung der Doktoranden in Angelegenheiten ihres Ausbildungsplans. Diese Aufgabe wird von einem besonderen Koordinator wahrgenommen. Die wissenschaftliche Betreuung der Doktorarbeiten erfolgt ausschließlich durch den
Erst- und den Zweitbetreuer der Arbeiten; Letzterer wird bereits bei Ausbildungsbeginn festgelegt.

Besondere Zuständigkeiten bestehen darüber hinaus für die Betreuung der ausländischen Gastwissenschaftler, die Öffentlichkeitsarbeit und den Datenschutz. Die aktuellen Ansprechpartner finden sich auf den Internetseiten des Instituts.

\section{Arbeitstreffen und Vorträge}

Die Mitarbeiter der strafrechtlichen Abteilung treffen sich regelmäßig einmal in der Woche zu einem festen Termin. Dabei finden Projekt- und Arbeitsbesprechungen, aber auch Vorträge und Diskussionen - vor allem von ausländischen Gästen - statt.

Die Vorträge sind institutsöffentlich und werden auch von den Gastwissenschaftlern im Hause gern besucht. Diese „Mittwochsvorträge“ werden durch weitere Vortragsveranstaltung an Abendterminen, zu denen auch auswärtige Gäste eingeladen sind, ergänzt.

\section{KRIMINOLOGISCHE ABTEILUNG}

Die Kriminologische Abteilung des Max-PlanckInstituts für ausländisches und internationales Strafrecht besteht seit 1970. Sie wurde mit der Berufung von Professor Günther Kaiser zum Direktor des Instituts und zum Leiter der Kriminologischen Forschungsgruppe als zweite Abteilung neben der Strafrechtlichen Forschungsgruppe gegründet. Mit Inkrafttreten der neuen Institutssatzung vom 29. Juni 2007 wurde die Kriminologische ebenso wie die Strafrechtliche Forschungsgruppe in ,Abteilung' umbenannt.

Die kriminologische Forschung ist projektbezogen organisiert. Im monatlichen Turnus findet unter der Leitung von Professor Hans-Jörg Albrecht eine Gruppensitzung mit allen wissenschaftlichen Mitarbeiterinnen und Mitarbeitern statt, die in die Forschungsarbeit eingebunden sind. Die Sitzung dient der Information über Entwicklung und Stand der einzelnen Untersuchungen, der Diskussion projektbezogener Fra- gen sowie der allgemeinen Planung der wissenschaftlichen Forschung.

Die Betreuung der Gastforscher liegt in der Hand von Volker Grundies. Die Öffentlichkeitsarbeit wird von Gunda Wößner betreut. Für Angelegenheiten der internationalen Beziehungen sowie der Forschungsförderung mit dem besonderen Schwerpunkt EU-Programme ist Dietrich Oberwittler zuständig. Michael Kilchling kümmert sich um Fragen der Nachwuchsförderung und die Beziehungen zur Albert-Ludwigs-Universität Freiburg, insbesondere im Hinblick auf die Lehrverpflichtungen an der Juristischen Fakultät. Die Organisation der International Max Planck Research School on Retaliation, Mediation and Punishment (IMPRS-REMEP) obliegt Carolin F. Hillemanns. Verantwortlich für Datensicherheit und Archivierung sind Volker Grundies und Jochen Jähnke. Der Sachbereich Veröffentlichungen fällt in die Zuständigkeit von Michael Knecht und Ulrike Auerbach. 


\section{B. Bibliothek}

Mit nunmehr rund 400.000 Medieneinheiten dürfte die Bibliothek des Max-Planck-Instituts für ausländisches und internationales Strafrecht in Europa die größte juristische Spezialbibliothek für strafrechtliche und kriminologische Literatur sein. Im Berichtszeitraum 2006-2007 konnte der Bibliotheksbestand, nicht zuletzt dank Sondermitteln der MPG, weiter ausgebaut werden. Gleichzeitig wurde der Aufbau der digitalen Bibliothek fortgeführt. Der internationale Stellenwert der Bibliothek spiegelt sich in der erneut gestiegenen Anzahl von Nutzern. Aber schon der Zuwachs des Bibliotheksbestandes spricht für sich.

So belief sich der Gesamtbestand der Bibliothek zum 31.12.2007 auf rund 397.000 Bände (31.12.2005: ca. 380.000 Bände). Rund 100.400 Bände davon sind Zeitschriftenbände. Der Zuwachs der Bibliothek betrug im Jahr 2007 (2006) 9.141 (9.316) Bände (gegenüber 8.170 bzw. 8.621 Bänden in den beiden Vorjahren). Hiervon sind 6.401 (5.884) Bände Monographien oder Fortsetzungen und 2.740 (3.432) Bände Zeitschriftenbände. Für Neuerwerbungen und Einband wurden im Jahr 2007 (2006) 629.832,14 EUR (641.712,00 EUR) aufgewendet.

Erneut konnte das Angebot an digitalen juristischen und kriminologischen Informationen und Volltexten, die den Nutzern im Intranet zugänglich gemacht werden, stark erweitert werden. Zu nennen sind hier insbesondere die Angebote, die dank der sogenannten „Nationallizenzen“ - einer von der DFG geförderten Initiative zur Versorgung der Wissenschaft mit elektronischen Fachinformationen - zur Verfügung gestellt werden. Daneben hält die Bibliothek 132 Zeitschriften mit lokalen Lizenzen digital bereit. Die in der Bibliothek vorhandenen digitalen wie herkömmlichen Publikationen werden im elektronischen Katalog (Web-OPAC) nachgewiesen. Die Funktionalität dieser Nachweise reicht aber weit über die traditionellen Besitz- und Standortnachweise hinaus: Mit Hilfe der im Katalog des Südwestdeutschen Bibliotheksverbundes eingespielten Inhaltsverzeichnisse, die für einen Großteil der neueren Bücher vorliegen, gibt der Web-OPAC unmittelbar Auskunft über die Inhalte dieser Publikationen; zudem kann bei digitalen Publikationen vom Katalog aus direkt auf die Volltexte zugegriffen werden.

Während die Bibliothek im Jahr 2006 von 867 externen Benutzern - Tagesgäste und Institutsgäste - in Anspruch genommen wurde, erhöhte sich diese Zahl im Jahr 2007 auf 1.348 externe Benutzer. Dass trotzdem die Qualität der Betreuung beibehalten werden konnte, wurde durch Optimierung der Arbeitsabläufe erreicht (Gruppeneinführungen, Info-Blätter zur Bibliotheksbenutzung, Einsatz einer elektronischen Lesesaalverwaltung). Allerdings sind mit diesen Benutzerzahlen die Kapazitätsgrenzen der Bibliothek insoweit erreicht, als nur ein beschränktes Platzangebot in den Lesesälen vorhanden ist und auch die derzeitige personelle Situation keinen weiteren Zuwachs zulässt.

Im Berichtszeitraum wurden neben den Tätigkeiten im laufenden Bibliotheksbetrieb wieder mehrere (Sonder-)Projekte durchgeführt. So wurde mit dem Aufbau der Abteilung „Europarecht“ der wachsenden Bedeutung des am Institut vertretenen Forschungsschwerpunktes „Europäisches Strafrecht" Rechnung getragen. Das im Herbst 2004 mit Sondermitteln der MPG begonnene Projekt „Retrospektive Konversion des Kartenkatalogs" wurde weiter vorangetrieben, indem im Berichtszeitraum die Katalogisierung von 21.381 Buchtiteln aus den Altbeständen im elektronischen Bibliothekssystem erfolgte. Darüber hinaus erfolgte die Erfassung von 31.175 Zeitschriftenbänden. In einem - von der MPG wiederum mit Sondermitteln geförderten - weiteren Projekt konnten die bisher noch nicht in elektronischer Form vorliegenden Institutspublikationen digitalisiert werden. Insgesamt wurden von August bis Dezember 2007 rund 89.000 Buchseiten der in den Schriftenreihen „Beiträge und Materialien aus dem Max-Planck-Institut für ausländisches und internationales Strafrecht“ und „Kriminologische Forschungsberichte aus dem Max-Planck-Institut für ausländisches und internationales Strafrecht" erschienenen Büchern eingescannt, nachbearbeitet und als pdf-Dokumente generiert. 


\title{
C. Herausgabe von Buchreihen und Zeitschriften
}

\author{
1. Fortführung, Änderungen und Neugründungen von Buchreihen
}

Das Institut veröffentlichte bis zum Jahr 2004 die Forschungsergebnisse der strafrechtlichen und der kriminologischen Forschungsgruppe vornehmlich in mehreren Buchreihen im Eigenverlag. Mit der Amtsübernahme durch Prof. Dr. Ulrich Sieber und dem Erscheinen des 100. Bandes der strafrechtlichen Forschungsreihe wurde eine Reihe von Veränderungen vorgenommen, die zwei Zielen dienten: Erstens sollten die bisher in einer Vielzahl von unterschiedlich gestalteten Reihen sowohl hausintern als auch bei anderen Verlagen herausgegebenen Publikationen so weit wie möglich in einer institutseigenen Schriftenreihe mit parallelen Unterreihen konzentriert werden, um die Institutsarbeiten auf der Grundlage einer einheitlichen Publikationsgestaltung besser erkennbar und das Institut dadurch in der wissenschaftlichen Diskussion stärker sichtbar zu machen. Zweitens sollten die Forschungsergebnisse nicht mehr nur im Eigenverlag, sondern in Zusammenarbeit mit einem renommierten Wissenschaftsverlag publiziert und dadurch auch für neue Leserkreise erschlossen werden.

Seit dem Jahr 2005 sind die Publikationen beider Forschungsabteilungen nunmehr in Kooperation mit dem Verlag Duncker \& Humblot in der „Schriftenreihe des Max-Planck-Instituts für ausländisches und internationales Strafrecht" zusammengeführt.
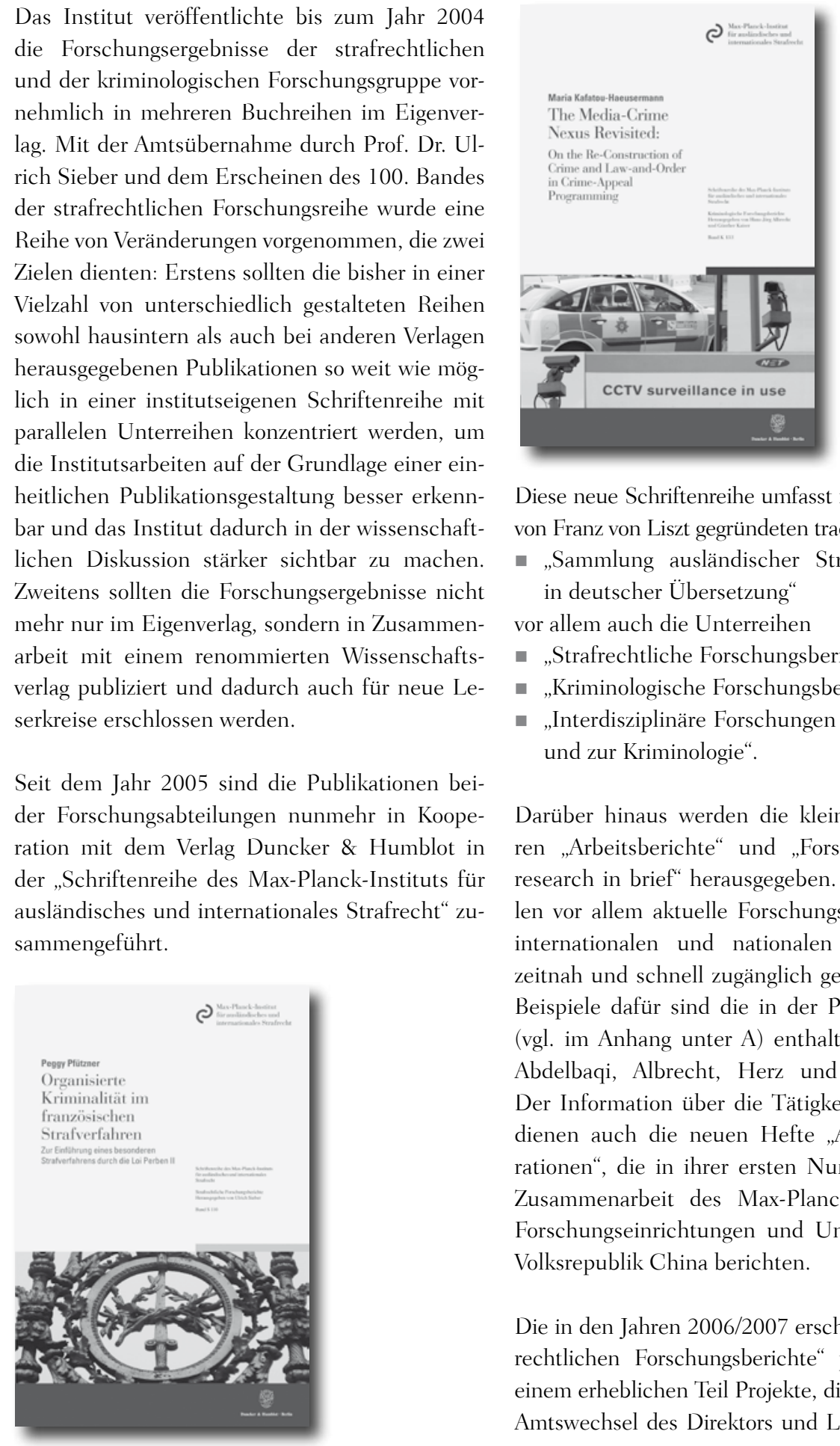

Diese neue Schriftenreihe umfasst neben der 1884 von Franz von Liszt gegründeten traditionsreichen

n „Sammlung ausländischer Strafgesetzbücher in deutscher Übersetzung"

vor allem auch die Unterreihen

v "Strafrechtliche Forschungsberichte“,

- „Kriminologische Forschungsberichte“ sowie

- „Interdisziplinäre Forschungen zum Strafrecht und zur Kriminologie“.

Darüber hinaus werden die kleineren Broschüren „Arbeitsberichte“ und „Forschung aktuell/ research in brief" herausgegeben. Mit ihnen sollen vor allem aktuelle Forschungsergebnisse der internationalen und nationalen Öffentlichkeit zeitnah und schnell zugänglich gemacht werden. Beispiele dafür sind die in der Publikationsliste (vgl. im Anhang unter A) enthaltenen Titel von Abdelbaqi, Albrecht, Herz und Schäfer/Paoli. Der Information über die Tätigkeit des Instituts dienen auch die neuen Hefte „Auslandskooperationen", die in ihrer ersten Nummer über die Zusammenarbeit des Max-Planck-Instituts mit Forschungseinrichtungen und Universitäten der Volksrepublik China berichten.

Die in den Jahren 2006/2007 erschienenen „Strafrechtlichen Forschungsberichte“ publizierten zu einem erheblichen Teil Projekte, die noch vor dem Amtswechsel des Direktors und Leiters der straf- 
rechtlichen Abteilung begonnen, jedoch unter dem neuen Direktor weiterbearbeitet und fertig gestellt wurden. Die veröffentlichten „Kriminologischen Forschungsberichte" repräsentieren wichtige Schwerpunkte des kriminologischen Forschungsprogramms. Zwei Bücher der Reihe „Interdisziplinäre Forschungen aus Strafrecht und Kriminologie“ publizieren Beiträge zu Grundlagenfragen des Strafrechts und der Kriminologie; der eine
Band dokumentiert die Feierlichkeiten des Amtswechsels am Max-Planck-Institut im Jahr 2004, der andere Band das Kolloquium zum 90. Geburtstag des Institutsgründers Prof. Dr. Hans-Heinrich Jescheck im Jahr 2005. In der „Sammlung ausländischer Strafgesetzbücher in deutscher Übersetzung erschienen deutsche Übersetzungen des StGB der Russischen Föderation, des finnischen Strafgesetzes sowie der bulgarischen StPO.

\section{Strafrechtliche Forschungsberichte}

\section{Hrsg. Ulrich Sieber}

Band S 82.9 Eser/Sieber/Arnold (Hrsg.), Strafrecht in Reaktion auf Systemunrecht. Vergleichende Einblicke in Transitionsprozesse. Teilband 9: China (Thomas Richter). Berlin 2006, 267 S.

Band S 82.10 Eser/Sieber/Arnold (Hrsg.), Strafrecht in Reaktion auf Systemunrecht. Vergleichende Einblicke in Transitionsprozesse. Teilband 10: Südkorea (Byung-Sun Cho, Carolin Holzapfl, Thomas Richter). Berlin 2006, 126 S.

Band S 82.11 Eser/Sieber/Arnold (Hrsg.), Strafrecht in Reaktion auf Systemunrecht. Vergleichende Einblicke in Transitionsprozesse. Teilband 11: Chile/Uruguay (Salvador A. Millaleo Hernández/Gonzalo D. Fernández). Berlin 2007, 643 S.

Band S 95.7 Eser/Sieber/Kreicker (Hrsg.), Nationale Strafverfolgung völkerrechtlicher Verbrechen. National Prosecution of International Crimes. Teilband 7: Völkerstrafrecht im Ländervergleich (Helmut Kreicker). Berlin 2006, 424 S.

Band S 106 Clivia von Dewitz, NS-Gedankengut und Strafrecht. Die §§ 86, 86a StGB und § 130 StGB zwischen der Abwehr neonazistischer Gefahren und symbolischem Strafrecht. Berlin 2006, 304 S.

Band S $107 \quad H e l m u t$ Kreicker, Völkerrechtliche Exemtionen. Grundlagen und Grenzen völkerrechtlicher Immunitäten und ihre Wirkungen im Strafrecht. Berlin 2007, 1538 S. (S 107, 2 Teilbände)

Band S $108 \quad$ Konstanze Jarvers, Massen- und Kleinkriminalität vor dem italienischen Friedensrichter. Neue Wege durch alternative Erledigungsmöglichkeiten und Sanktionsformen. Berlin 2007, 308 S.

Band S 109 Johanna Rinceanu, Völkerstrafrecht in Rumänien. Berlin 2008, 316 S.

Band S $110 \quad$ Peggy Pfützner, Organisierte Kriminalität im französischen Strafverfahren. Zur Einführung eines besonderen Strafverfahrens durch die Loi Perben II. Berlin 2008, 302 S.

Band S 111

Silvia Tellenbach (Hrsg.), Die Rolle der Ehre im Strafrecht. Berlin 2007, 813 S.

\section{Kriminologische Forschungsberichte \\ Hrsg. Hans-Jörg Albrecht und Günther Kaiser †}

Band K 125 Didem Aydin, Die strafrechtliche Bekämpfung von Hassdelikten in Deutschland und in den Vereinigten Staaten von Amerika. edition iuscrim, Freiburg/Br. 2006, 519 S.

Band K 130 Carsten Schäfer/Letizia Paoli, Drogenkonsum und Strafverfolgungspraxis. Berlin 2006, 455 S.

Band K $131 \quad$ Stefan Schulz, Beyond Self-Control. Analysis and Critique of Gottfredson \& Hirschi's General Theory of Crime (1990). Berlin 2006, 306 S. 
Band K 132 Gunda Wößner, Typisierung von Sexualstraftätern. Berlin 2006, 270 S.

Band K 133 Maria Kafatou-Haeusermann, The Media-Crime Nexus. Berlin 2007, 539 S.

Band K $134 \quad$ Gundula Schäfer-Vogel, Gewalttätige Jugendkulturen - Symptom der Erosion kommunikativer Strukturen. Berlin 2007, 590 S.

Band K $135 \quad$ Stefanie Tränkle, Im Schatten des Strafrechts. Berlin 2007, 392 S.

Band K 136 Tim Lukas (ed.), Crime Prevention in High-Rise Housing. Berlin 2007, 132 S.

Band K $137 \quad$ Evelyn Shea, Why work? Berlin 2007, 195 S.

Interdisziplinäre Forschungen aus Strafrecht und Kriminologie

2006/2007

Band I 12 Albrecht/Sieber (Hrsg.), Perspektiven der strafrechtlichen Forschung. Berlin 2006, 87 S.

Band I 13 Hans-Jörg Albrecht/Jan-Michael Simon/Hassan Rezai/Holger-C. Rohne, Ernesto Kiza (Hrsg.), Conflicts and Conflict Resolution in Middle Eastern Societies. Berlin 2006, 668 S.

Band I 14 Sieber/A/brecht(Hrsg.), Strafrecht und Kriminologie unter einem Dach. Kolloquium zum 90. Geburtstag von Professor Dr. Dr. h.c. mult. Hans-Heinrich Jescheck. Berlin 2006, 202 S.

Sammlung ausländischer Strafgesetzbücher in deutscher Übersetzung

2006/2007

Band G 108 Strafgesetzbuch der Russischen Föderation. Deutsche Übersetzung und Einführung von FriedrichChristian Schroeder. 2., aktual. Auflage. Berlin 2007, 260 S.

Band G 116 Das finnische Strafgesetz - Rikoslaki - Strafflag. Dreisprachige Ausgabe. Übersetzung und Einführung von Karin Cornils, Dan Frände und Jussi Matikkala. Berlin 2006, 563 S.

Band G 117 Die bulgarische Strafprozessordnung. Deutsche Übersetzung von Irina Gencheva. Einführung von Lazar Gruev. Berlin 2007, 196 S.
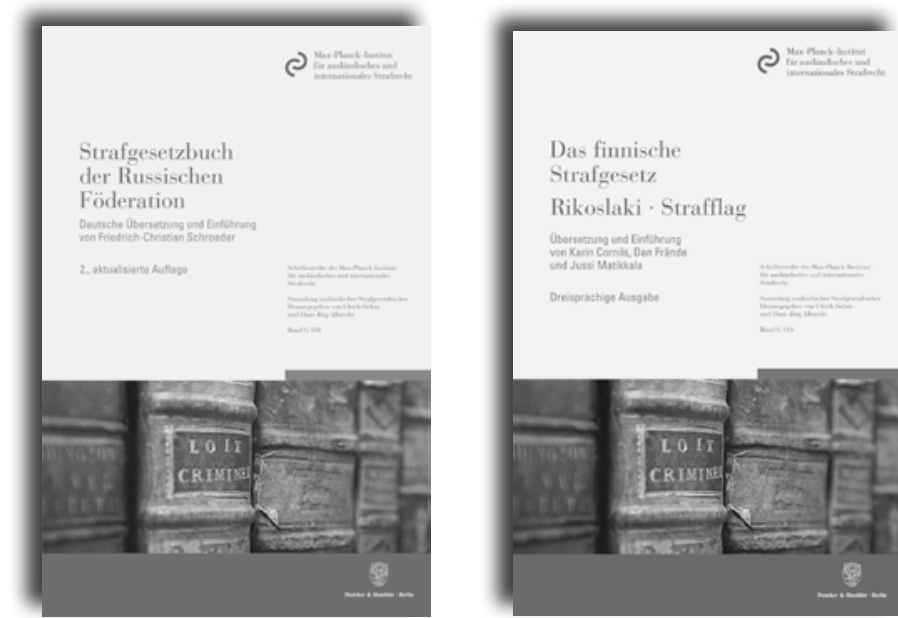


\section{Zeitschriften}

Die Direktoren des Instituts wirken an der Herausgabe verschiedener Zeitschriften mit, die unter IV.B. jeweils genannt sind. Besondere Bedeutung haben dabei die folgenden Zeitschriften, deren Redaktion im Institut erfolgt.

\section{Auslandsrundschau der Zeitschrift für die gesamte Strafrechtswissenschaft (ZStW) Verlag Walter de Gruyter, Berlin}

Seit dem Jahr 2006 wird die Zeitschrift von Ulrich Sieber und Hans-Jörg Albrecht herausgegeben. Die Schriftleitung liegt bei Ulrich Sieber. Barbara Huber ist für die Redaktion verantwortlich. Die 1881 von Franz von Liszt und Adolf Dochow gegründete Zeitschrift ist in Deutschland das zentrale wissenschaftliche Periodikum für ausländisches Strafrecht. Sie unterrichtet die strafrechtliche Fachwelt über neueste Rechtsentwicklungen auf dem Gebiet des nationalen, internationalen und supranationalen Strafrechts, analysiert Gesetzgebung, Rechtsanwendung und Reformen und informiert über wichtige Konferenzen und deren Ergebnisse. Es kommen deutsche und ausländische Strafrechtswissenschaftler und Praktiker zu Wort; die Veröffentlichungssprache ist deutsch.

\section{Monatsschrift für Kriminologie und Strafrechtsreform (MschrKrim), Heymanns Verlag Köln}

Die Zeitschrift wird mitherausgegeben von Hans-Jörg Albrecht; die Schriftleitung liegt bei ihm. Die Redaktion führt Ulrike Auerbach. Die Monatsschrift ist die zentrale wissenschaftliche Publikation der deutschsprachigen Kriminologie und ihrer Nachbardisziplinen. Sie erschien im Jahr 2007 im 90. Jahrgang. Neben der systematischen Berichterstattung über Forschungsergebnisse und kriminalpolitische Entwicklungen zielt die Monatsschrift auf eine kritische Begleitung von Strafrecht, Strafrechtspraxis sowie Kriminalpolitik und bildet zudem ein Forum, das den Austausch über die Grenzen der wissenschaftlichen Disziplinen hinaus und über sprachliche Grenzen hinweg ermöglicht.

\section{European Journal of Crime, Criminal Law and Criminal Justice, \\ Brill Academic Publishers, Leiden, Niederlande}

Die Zeitschrift wird auf der Basis einer Kooperation zwischen dem Max-Planck-Institut für ausländisches und internationales Strafrecht und der Universität Tilburg (Niederlande) von Hans-Jörg Albrecht, Cyrill Fijnaut (Tilburg), Günther Kaiser † und Ulrich Sieber herausgegeben. Die Redaktion wird von Michael Knecht betreut. Das englischsprachige Journal ist 2007 im 15. Jahr erschienen. Es konzentriert sich auf Forschungen zur Kriminalität, zum Strafrecht und zur Strafjustiz in Europa aus einer vergleichenden wie auch europäischen Perspektive und hat sich zu einem der wichtigsten europäischen Foren für den wissenschaftlichen Austausch in den erfassten Bereichen entwickelt. Neben europäischen Themen im engeren Sinne werden auch solche Beiträge aus den Nachbarregionen Europas erfasst, die Bezüge zu Europa oder zur Europäischen Union herstellen.

\section{eucrim - The European Criminal Law Associations' Forum}

Die Zeitschrift wird vom Max-Planck-Institut durch Ulrich Sieber in Zusammenarbeit mit der Vereinigung für europäisches Strafrecht e.V. seit 2006 regelmäßig herausgegeben. Die Redaktion obliegt Thomas Wahl. eucrim wirkt dem von der europäischen Strafrechtswissenschaft beklagten Informationsdefizit im Bereich des Europäischen Strafrechts entgegen, insbesondere durch die Analyse der aktuellen strafrechtsrelevanten Entwicklungen in der Europäischen Union und im Europarat sowie durch die Veröffentlichung von Beiträgen zu Schwerpunktthemen. Die Zeitschrift ist ein neues multimediales Informationssystem für Wissenschaftler, Praktiker und Politiker, das zwar auch als Printversion gedruckt, überwiegend jedoch online zur Verfügung gestellt wird. Die Online-Version hat zwei Jahre nach ihrem Start bereits ca. 1200 Abonnenten. Die Printversion erscheint in 2000 Exemplaren. 


\section{D. Öffentlichkeitsarbeit}

Die Öffentlichkeitsarbeit des Instituts verfolgt das Ziel einer möglichst umfassenden Vermittlung der Forschungstätigkeit an ein breites öffentliches ebenso wie an ein fachspezifisch interessiertes Publikum. Hierfür werden verschiedene Strategien bedarfsgerecht ein- und umgesetzt: An erster Stelle stehen die Publikationen der Schriftenreihe des Max-Planck-Instituts für ausländisches und internationales Strafrecht. Diese werden ergänzt durch die im Haus herausgegebenen Zeitschriften sowie Veröffentlichungen externer Verlage und elektronische Publikationen. Ein zentrales Instrument der Öffentlichkeitsarbeit ist für das Institut auch das Internet geworden. Dort wird auf Neuerscheinungen hingewiesen. Für alle Buchpublikationen stehen detaillierte Informationen, Bestellmöglichkeiten und zum Teil auch Aktualisierungen zum kostenlosen Abruf zur Verfügung. Die Veröffentlichungen aus der Reihe „Research in Brief / Forschung Aktuell“ werden über die Homepage auch im Volltext kostenlos abgegeben. Weiterhin werden für alle Projekte umfangreiche Darstellungen gepflegt, die interessierten Nutzern Informationen zu Inhalt, Forschungsstand und relevanten Publikationen bieten. Die derzeitige Version der Homepage wird ständig aktualisiert und gepflegt und spiegelt hierdurch die gegenwärtigen, am Institut laufenden Entwicklungen zeitnah wider.

Daneben wurden verschiedene Broschüren und Prospekte erstellt, die entweder projektspezifisch über die Forschungstätigkeit berichten oder projektübergreifend wichtige Entwicklungen des Instituts, wie z.B. die beiden neuen Research Schools, oder Forschungskooperationen aufgreifen. Somit können bei Veranstaltungen und auf Nachfrage entsprechende Materialien, die über das Institut und die hier stattfindende Forschung informieren, öffentlichkeitswirksam in Umlauf gebracht werden.

Die Öffentlichkeitsarbeit beinhaltet auch aktive Veröffentlichungen außerhalb des Instituts. So entstand z.B. ein ganzseitiger Namensbeitrag in der FAZ zu dem aktuellen Thema der Liechtensteiner Steuerermittlungen (vgl. Kapitel II.D.). Auch im Wissenschaftsmagazin Max-Planck-Forschung, das viermal im Jahr erscheint und in großer

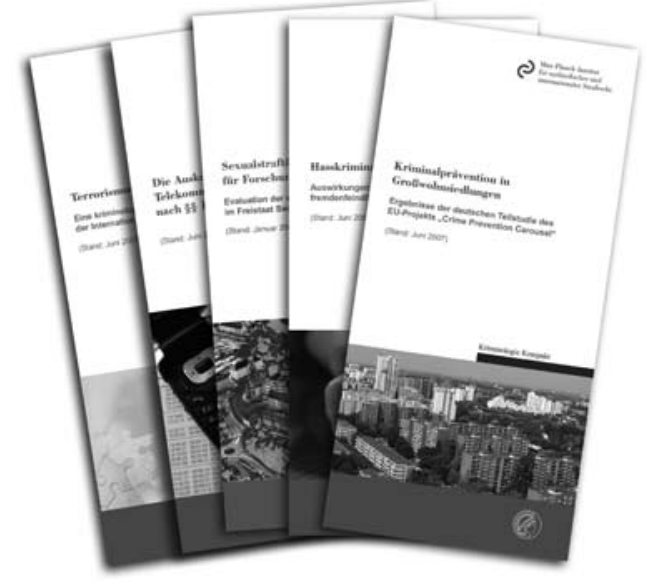

Auflage gedruckt wird, wurde über mehrere am Institut laufende Projekte berichtet. Im Jahr 2007 erschienen beispielsweise eine Reportage über Verbrecherjagd im Datennetz und ein Bericht über das Institutsprojekt zur Kriminalprävention in Großwohnsiedlungen.

Zu den allgemeinen PR-Strategien gehören auch die Organisation von internen (also am Institut selbst stattfindenden) und externen Vortragsveranstaltungen, Kolloquien und Tagungen, welche vom Institut mit ausgetragen werden. Große Bedeutung hierfür haben die hausinternen Vorträge erhalten, die sich in erster Linie an die Wissenschaftlerinnen und Wissenschaftler im Hause sowie die Institutsgäste richten, gleichwohl zum Teil auch öffentlich zugänglich waren. Zu den Referenten, die hierfür gewonnen werden konnten zählten u.a. Prof. Dr. Dr. h.c. mult. Günther Jakobs und Prof. David Wall, University of Leeds. Daneben wurden zahlreiche Tagungen und Symposien am Max-Planck-Institut durchgeführt, so z.B. der Workshop „Reforming Criminal Justice: Key Issues of Contemporary Criminal Procedure“ im Oktober 2007, das interdisziplinäre Seminar „Phishing, Filesharing, War-Driving - aktuelle Cybercrime-Fragestellungen“ im Januar 2007, eine Sondersitzung des Fachausschusses Strafrecht der Deutschen Gesellschaft für Recht und Informatik, oder das Kolloquium zum 40-jährigen Bestehen des Max-Planck-Instituts für ausländisches und internationales Strafrecht am 21. Juli 
2006 zu Strafrechtsvergleichung und vergleichender Kriminologie. Bei zahlreichen Tagungen und Kolloquien, die außerhalb des Max-PlanckInstituts stattfinden, ist das Institut ebenfalls an der Austragung diverser Veranstaltungen beteiligt, wie dies beispielsweise beim Expertenkolloquium „Evaluation der Sozialtherapie im Justizvollzug“ im November 2007 der Fall war, das zusammen mit der Kriminologischen Zentralstelle (KrimZ) in Wiesbaden veranstaltet wurde.

Außerdem konnten Besuchergruppen aus aller Welt am Institut willkommen geheißen werden. Im September 2007 besuchte eine Gruppe von Studierenden der Universität Kumamoto, Japan, und der dort angesiedelten Graduate School für Juristen das Institut. Im Januar und Februar 2007 informierte sich eine Delegation türkischer Polizeirechtsexperten über aktuelle Entwicklungen in der kriminologisch orientierten Polizeiforschung. Am 16. Oktober 2006 besuchte eine Delegation höherer Beamter aus Justizministerien und obersten Gerichten der EU-Mitglieds- und -Beitrittsländer zusammen mit Bediensteten der EU-Kommission und des Rates der EU, des Europäischen Gerichtshofs und von Eurojust das Max-Planck-Institut für ausländisches und internationales Strafrecht in Freiburg. Daneben wurde vielen weiteren größeren und kleineren Besuchergruppen vermittelt, welche Forschungsarbeiten gegenwärtig die Tätigkeiten des Instituts bestimmen.
Da eine erfolgreiche Öffentlichkeitsarbeit für das Institut zunehmend Bedeutung gewinnt, wurde im Berichtszeitraum ein verstärkter und kontinuierlicher Ausbau der PR-Tätigkeit vorangetrieben. Höhepunkt dieser Aktivitäten war die Teilnahme des Instituts an der im Jahr 2007 veranstalteten Wissenschaftsmeile im Rahmen der 550-Jahr-Feier der Albert-Ludwigs-Universität Freiburg rund um die Freiburger Universität. Vom 11. bis 14. Juli 2007 präsentierte sich das Max-Planck-Institut für ausländisches und internationales Strafrecht zusammen mit dem Freiburger Max-Planck-Institut für Immunbiologie und der Arbeitsgruppe Feuerökologie des Mainzer Max-Planck-Instituts für Chemie in einem eigens für die Max-Planck-Institute entworfenen und angefertigten Pavillon.

Hier präsentierte das Institut Wissenschaft „zum Anfassen“ in Form von Postern, Plakaten, Broschüren sowie interaktiven computergestützten Präsentationen. Auf dem Wissenschaftsmarkt stellten neben den Max-Planck-Instituten ca. 50 weitere Freiburger Institute und Abteilungen der Universität ihre Forschungstätigkeiten vor und zogen rund 30.000 Besucher an. Vor dem Hintergrund dieser Großveranstaltung organisierte das Max-Planck-Institut für ausländisches und internationales Strafrecht zusammen mit der Max-Planck-Gesellschaft in München ein MaxPlanck-Forum, welches am 11. Juli 2007 unter dem Titel „Trojaner gegen den Terror?” in den Räumlichkeiten der Deutschen Bank Freiburg

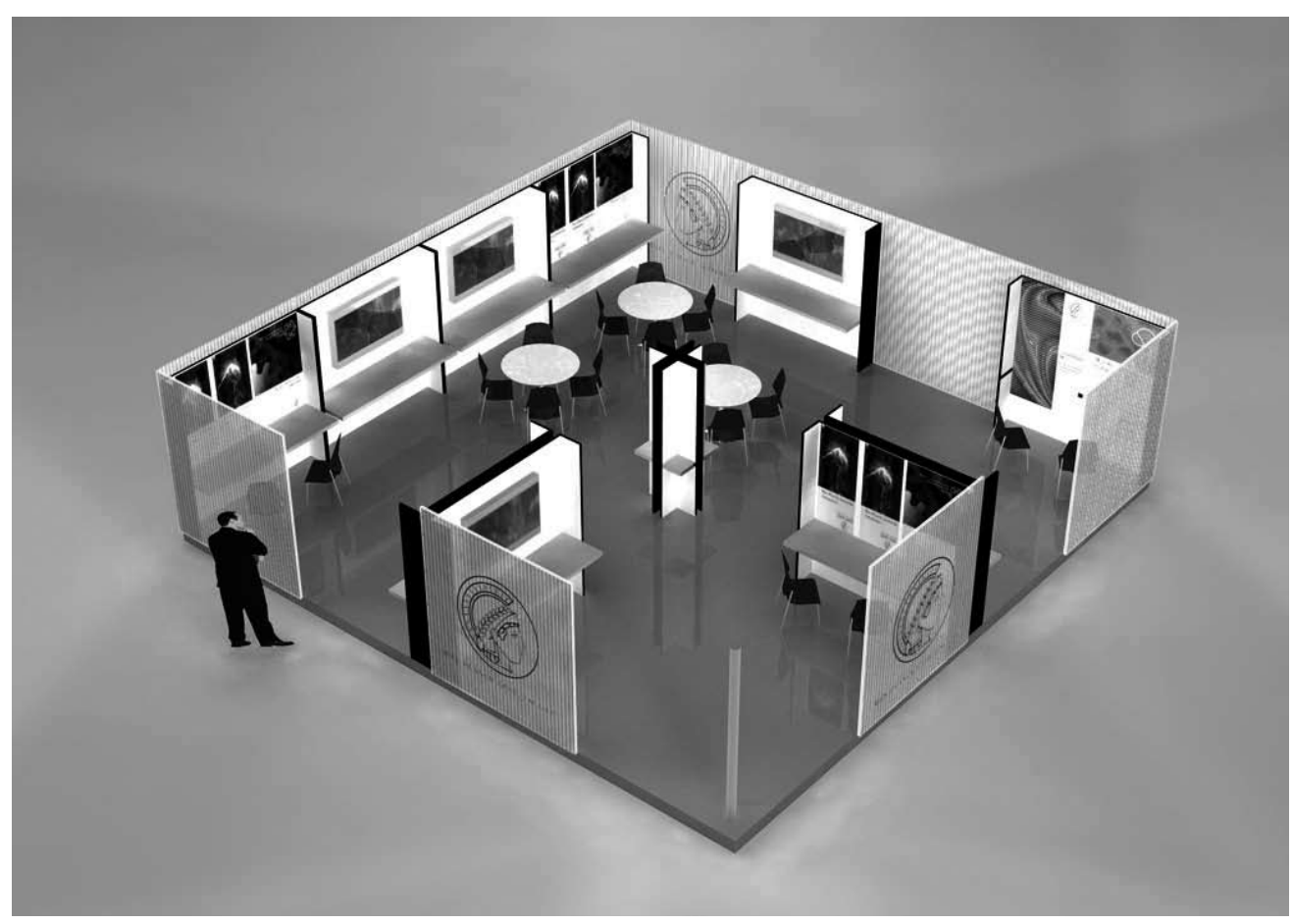

Pavillon der Max-Planck-Institute bei der Meile der Wissenschaft 


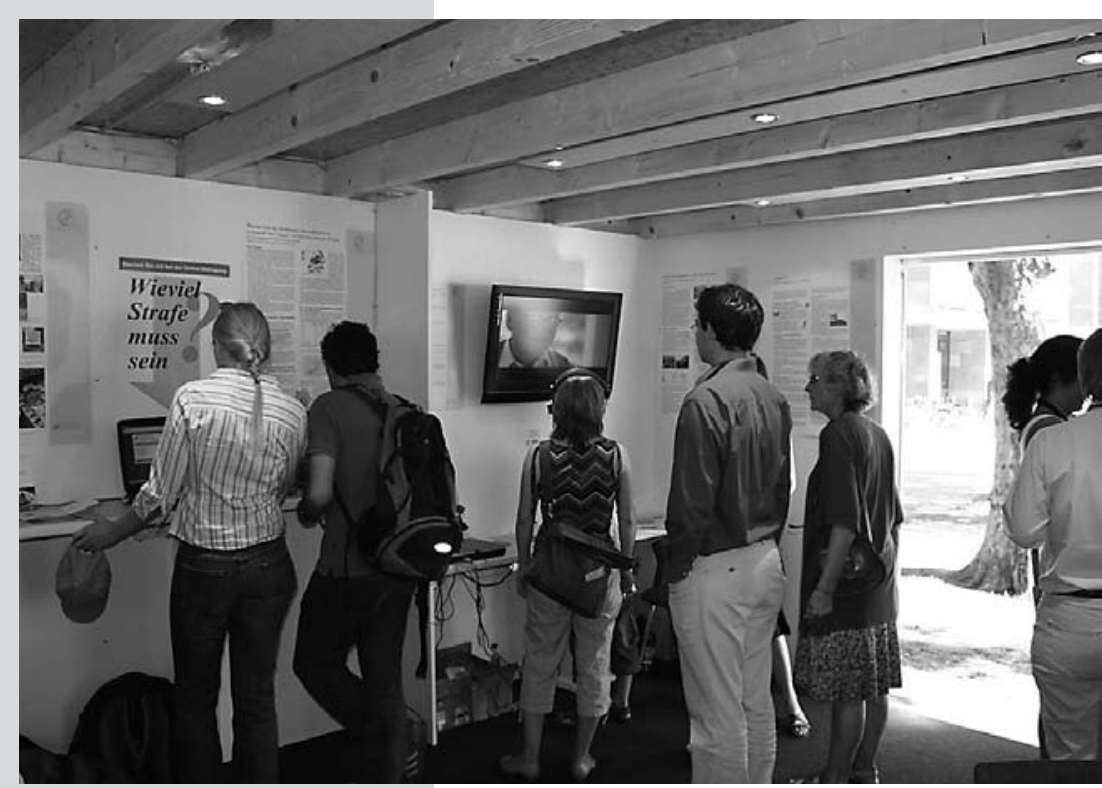

Besucher im Pavillon der MaxPlanck-Institute bei der Meile der Wissenschaft
In den letzten beiden Jahren hat das Institut insgesamt eine offensivere PR-Tätigkeit entwickelt. Dies zeigt sich vor allem im Umgang mit den Medien. Mittlerweile gibt es einen eigenen Presseverteiler, an den Pressemitteilungen versandt werden. Pressemitteilungen werden zunehmend eigeninitiativ für aktuelle politische Anlässe und gesellschaftspolitisch relevante Themen erstellt (so z.B. in der Vergangenheit zu den Themen Online-Überwachung, Rasterfahndung, Kindstötung). Diese Pressemitteilungen finden sich nunmehr auch online auf der Homepage. Die Zusammenarbeit mit der Max-Planck-Gesellschaft in München geht hier derweil Hand in Hand. Daneben bekommen die beiden Abteilungen regelmäßig Anfragen von Printmedien, Funk und Fernsehen. Diese Anfragen beziehen sich zum einen auf Interviews mit den Direktoren und den wissenschaftlichen Mitarbeitern, zum anderen auf die Vermittlung von Hintergrundinformationen durch die Experten des Instituts zu speziellen Themen. Hieraus resultierten im Berichtszeitraum zahlreiche Berichterstattungen, in denen auf Mitarbeiter des Instituts verwiesen wird, sowie Interviews in Tageszeitungen, wissenschaftlichen Zeitschriften, Rundfunk und Fernsehen. Exemplarisch seien hier die Kommentierung von Prof. Sieber zur Life-Übertragung der Urteilsverkündung des Bundesverfassungsgerichts in dem Verfahren über die Online-Duchsuchung für den Fernsehsender Phoenix genannt sowie die Expertentätigkeit von Prof. Albrecht für die Tagesthemen und dessen Teilnahme an der Phoenix-Runde. rigen Jubiläums der Universität Freiburg wurden an mehreren Freiburger Schulen Vorträge gehalten, die traditionsgemäß ansonsten nur in den Städten, in denen die Jahreshauptversammlung der Max-Planck-Gesellschaft abgehalten wird, angeboten werden. Die wissenschaftlichen Mitarbeiter des Instituts hielten zu insgesamt zehn verschiedenen Themen Schulvorträge an Freiburger Gymnasien. Auf größtes Interesse stießen mit jeweils drei Präsentationen der Vortrag „Hacker, Cracker, Bauernfänger - Strafrecht im Zeitalter des Internet“ und der Vortrag „Lucky Luke und der Psycho-Doc - Oder: Soll man gefährliche Straftäter behandeln oder wegsperren?". Auch auf den Jahreshauptversammlungen in Kiel (2007) und Frankfurt (2006) war das Institut mit zahlreichen Schulvorträgen vertreten.
Mit einem zunehmenden Fokus auf einer erfolgreichen Presse- und Öffentlichkeitsarbeit in einer Informationsgesellschaft folgt das Institut einer auch innerhalb der Max-Planck-Gesellschaft fortschreitenden Entwicklung. Diese schrieb im Jahr 2007 die Finanzierung für 14 PR-Stellen aus, um die sich alle 79 Max-Planck-Institute bewerben konnten. Auch das Max-Planck-Institut für ausländisches und internationales Strafrecht bewarb sich um die Finanzierung und war mit einer halben Stelle erfolgreich. Durch diese personelle Verstärkung soll die erfolgreiche Entwicklung der Presse- und Öffentlichkeitsarbeit weiter fortgeführt und ausgebaut werden. 


\section{E. EDV-Dienstleistungen}

Die Arbeit der EDV-Abteilung in den Jahren 2006 und 2007 war vor allem von drei Schwerpunkten geprägt. Diese betrafen erstens das Systemmanagement, zweitens den Ausbau der Kommunikationsinfrastruktur und drittens eine weitere Verbesserung der Computersicherheit.

\section{Systemmanagement}

Im Bereich Systemmanagement wurde die Konsolidierung der IT-Umgebung unter das „Active Directory“ der Firma Microsoft abgeschlossen. Hierdurch ist eine effektivere Verwaltung von Computern, Nutzern und Arbeitsgruppen möglich. Weiterhin erfolgte auch die Modernisierung

\section{Kommunikationsinfrastruktur}

Die Kommunikationsinfrastruktur wurde in weiten Teilen an den aktuellen Stand der Technik und die Bedürfnisse der Nutzer angepasst. Dies betrifft zunächst den E-Mail-Dienst, der von der EDV-Abteilung für Nutzer und Gäste angeboten wird. Im Jahr 2007 konnten alle bestehenden EMail-Konten auf einen neuen Mailserver migriert werden. Gegenüber dem alten Dienst, der seit 1999 auf wechselnder Hardware betrieben wurde, wird vor allem eine größere Systemstabilität erwartet. Zusätzlich bietet der neue Mailserver erstmals auch Groupware-Funktionalitäten, mit denen zum Beispiel Kalenderdienste zur Verfügung gestellt werden können. Schließlich konnte eine Software in Betrieb genommen werden, um den Betrieb von Mailinglisten am Institut zu gewährleisten. Dieses Angebot geht auf den steigenden Bedarf aus der Wissenschaft zurück, über E-Mail auch in größeren Gruppen Informationen austauschen zu können.

\section{Computersicherheit}

Der Bereich der Computersicherheit stellt seit vielen Jahren einen Schwerpunkt der Arbeit der EDV-Abteilung des Instituts dar. Eine Innovation war die Einführung eines Funk-Netzwerks des Bereichs der Hardware. Neben neuen Servern wurden Hochleistungsdrucker angeschafft, die nun im gesamten Institut zur Verfügung stehen. Zusätzlich wurden zwei neue Hochleistungsfarbdrucksysteme installiert und in Betrieb genommen

Im Frühjahr 2006 wurde eine neue Institutshomepage online geschaltet. Das Web-Angebot wird mit Hilfe eines Content-Management-Systems verwaltet, das gemeinsam mit anderen juristischen MaxPlanck-Instituten betrieben und konzipiert wurde. Im Lauf des Jahres 2007 konnte das Angebot zudem vollständig barrierefrei gestaltet werden.

Neben der IT-Infrastruktur im engeren Sinne war die EDV-Abteilung auch für andere elektronische Dienste des Instituts zuständig. So wurden etwa eine neue Telefonanlage sowie Komfort-Telefone in allen Büros in Betrieb genommen. Dies ermöglicht eine Integration von Telefondiensten auch in die IT-Systeme. Hierdurch wird nicht nur der Zugriff auf Adressbücher und Ablagefunktionen ermöglicht und die tägliche Büroarbeit erleichtert, sondern es werden auch Telefonumfragen unterstützt, die im Rahmen von einzelnen Forschungsprojekten in den Abteilungen durchzuführen sind.

am Institut im Berichtszeitraum. Damit besteht für Mitarbeiter und Gäste die Möglichkeit, auch drahtlos einen Internetzugang über das Institut zu nutzen. Anders als bei vielen anderen kabellosen 


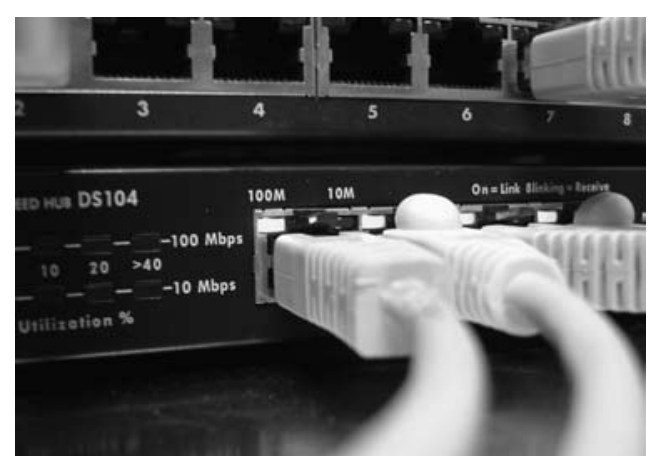

Netzwerken wurde großer Wert auf die Netzwerksicherheit gelegt. Die gewählte Konfiguration entspricht den neuesten Standards und bietet Sicherheit auf höchstem Niveau.

Neben dem drahtlosen Zugang im Institut wurde von vielen Wissenschaftlern gefordert, eine Möglichkeit auch für den mobilen Zugriff z.B. auf Reisen zu schaffen. Eine Herausforderung besteht hierbei insbesondere in der Gewährleistung sowohl der Systemsicherheit als auch des Zugriffsschutzes. 2006 konnte eine komfortable Zugangsmöglichkeit in Betrieb genommen werden, mit der eine Arbeit auch außerhalb des Instituts möglich ist. Der Zugang ist vollständig verschlüsselt und verwendet auf der höchsten Sicherheitsstufe sogenannte RSA-Token als Einmal-Passwörter.

Weitere Arbeiten der EDV-Abteilung mit Bezug zur Computersicherheit, die im Berichtszeitraum vorgenommen wurden, betreffen den Betrieb eines eigenen Update-Servers, mit dem sichergestellt werden kann, dass Rechner im Institut regelmäßig mit sicherheitsrelevanten Aktualisierungen versorgt werden können sowie den Aufbau von Zertifizierungs- und Registrierungsstellen für Sicherheitszertifikate. Derartige Zertifikate spielen eine zentrale Rolle in der modernen Kommunikation. 


\section{F. Forschungsförderung}

Ein wichtiges Element der Forschungsplanung des Instituts ist die Beteiligung an externen Förderprogrammen der allgemeinen öffentlich finanzierten Forschungsförderung sowie an den internen Sonderprogrammen der MPG zur Förderung der Spitzenforschung an den Max-Planck-Instituten, insbesondere dem Innovationsfonds des Präsidenten der MPG. Auch ist die punktuelle Erschließung von Drittmitteln seitens privater Institutionen insbesondere durch Stiftungen von Bedeutung. Das Institut verfolgt bei der Forschungsförderung drei programmatische und forschungsstrategische Ziele.

- Zum Ersten sind Schwerpunktsetzungen im Rahmen aktueller Förderprogramme von Einrichtungen zur Forschungsförderung Instrumente zur Forschungsinnovation. Dies gilt für die Formulierung neuer Probleme und wissenschaftlicher Fragestellungen sowie für den damit verbundenen Bedarf an methodischer Weiterentwicklung. Speziell die Forschungsrahmenprogramme der EU sind darüber hinaus auf das Entstehen neuer Forschungskooperationen und Netzwerke ausgerichtet und damit für das Forschungskonzept des Instituts von besonderem Nutzen.

- Zum Zweiten stellt die Evaluation des Antrags, die bei Förderprogrammen der allgemeinen Forschungsförderung regelmäßig stattfindet, ein bedeutendes Instrument zur Feststellung der Qualität des Forschungsvorhabens und somit von Exzellenz dar. Dies gilt insbesondere für die Bewilligung von Anträgen durch - zumeist international besetzte - Gutachtergremien in den Forschungsrahmenprogrammen der EU und den maßgebenden Institutionen in Deutschland, wie etwa der DFG, der Volkswagen- oder der FritzThyssen-Stiftung, sowie in besonderem Maße für den Innovationsfonds des Präsidenten der MPG.

- Zum Dritten ist die Beteiligung an Förderprogrammen und die Durchführung von Forschungsaufträgen aus der Politik und Praxis auf nationaler, europäischer und internationaler Ebene ein Weg, um unabhängige Ergebnisse aus der Grundlagenforschung in die Praxis einfließen zu lassen.

Das Institut profitiert von unterschiedlichen Formen der Forschungsförderung. Die größte Bedeutung kommt - inhaltlich wie auch im Hinblick auf den Förderungsumfang - unmittelbar projektbezogenen Programmen zu. Bei den externen Förderprogrammen ist in diesem Bereich die Förderung auf europäischer Ebene von besonderem Gewicht und zwar speziell durch die Kommission. Bei der internen Förderung durch die Sonderprogramme der MPG für die Spitzenforschung an ihren Instituten fällt in dem Berichtszeitraum insbesondere die Bewilligung des strafrechtsvergleichenden Projekts „Max Planck Systematik zum Strafrechtsvergleich“ aus dem Innovationsfonds des Präsidenten der MPG mit einer Laufzeit von 2008 bis 2012 ins Gewicht.

\begin{tabular}{|c|c|c|c|}
\hline Zuwendungsgeber & 2006 & 2007 & Gesamt \\
\hline Bund & $65.421 €$ & $52.244 €$ & $117.664 €$ \\
\hline Länder & $70.000 €$ & $76.160 €$ & $146.160 €$ \\
\hline EU & $55.664 €$ & $132.940 €$ & $188.604 €$ \\
\hline DFG & $53.828 €$ & $43.568 €$ & $97.396 €$ \\
\hline Stiftungen & - & $26.543 €$ & $26.543 €$ \\
\hline sonstige & $152.826 €$ & $108.551 €$ & $261.377 €$ \\
\hline Gesamteinnahmen Drittmittel & $397.738 €$ & $440.006 €$ & $837.744 €$ \\
\hline
\end{tabular}

Tabelle:

Herkunft der Drittmittel im Berichtszeitraum 2006/07 
Die personenbezogene Förderung in Form von Doktoranden- und Postdoc-Stipenden ermöglicht die Planung und Realisierung zahlreicher Einzelprojekte. Das gilt auch für größere Projekte aus Fördermitteln der DFG, die auf Weiterqualifizierung ausgerichtet sind. Für das Institut ist diese Form der Förderung die einzige Möglichkeit, von Fördermitteln der DFG zu profitieren, da projektbezogene DFG-Programme nach den entsprechenden Richtlinien an erster Stelle den Universitäten zugute kommen sollen.

Von Bedeutung sind darüber hinaus Forschungsaufträge staatlicher und überstaatlicher Institutionen. Das betrifft auf nationaler Ebene zahlreiche
Projekte im Auftrag von verschiedenen Bundesbzw. Landesministerien sowie der Landesmedienanstalten. Auf europäischer Ebene fällt in dem Berichtszeitraum insbesondere die Studie über die Reform des Europäischen Strafrechts ins Gewicht. Darüber hinaus wurden vor allem Studien im Auftrag des Europarats verfasst.

Ferner eröffneten Drittmittel - insbesondere aus Mitteln des DAAD und der Alexander-vonHumboldt-Stiftung - dem Institut einen größeren Spielraum für die Forschung, vor allem zur Durchführung internationaler Tagungen und Konferenzen im In- und Ausland, die das Institut über seinen Haushalt nicht bestreiten kann. 


\section{G. Fachbeirat und Kuratorium}

\section{DER FACHBEIRAT}

Unabhängige und regelmäßige Evaluation ist ein zentrales Element in der Forschungspolitik der Max-Planck-Gesellschaft. Die Forschungstätigkeit von Max-Planck-Instituten wird deshalb begleitet durch die in einem zweijährigen Abstand stattfindende Evaluation und Beratung durch externe Expertenkommissionen (Fachbeiräte).
Deren Mitglieder werden vom Präsidenten der Max-Planck-Gesellschaft auf der Grundlage von Vorschlägen aus den Instituten ernannt. Die Mitglieder des Fachbeirats des Max-Planck-Instituts für ausländisches und internationales Strafrecht repräsentieren die Strafrechtswissenschaften und die Kriminologie.

\section{Der Fachbeirat setzt sich derzeit wie folgt zusammen:}

- Prof. Dr. José Luis de la Cuesta, Universität San Sebastián/Spanien

- Prof. Dr. Tatjana Hörnle, Ruhr-Universität Bochum

- Prof. Dr. Karl-Ludwig Kunz, Universität Bern/Schweiz

- Prof. Dr. Lorenzo Picotti, Universität Verona/Italien

- Prof. Dr. Heinz Schöch, Universität München

- Prof. Dr. Steve Thaman, St. Louis University/USA

- Prof. Dr. Klaus Volk, Universität München

- Prof. Dr. Dr. h.c. mult. Luis Arroyo Zapatero, Universität Castilla-La Mancha, Ciudad Real/Spanien

- Prof. Dr. Dirk van Zyl Smit, University of Nottingham/Großbritannien

Bis Anfang 2008 gehörten dem Fachbeirat die folgenden Personen an:

- Prof. Dr. Heike Jung, Universität Saarbrücken (Vorsitzender)

- Prof. Dr. Günter Albrecht, Universität Bielefeld

- Prof. Dr. Knut Amelung, Universität Dresden

- Prof. Dr. Cyrille Fijnaut, Universität Tilburg/Niederlande

- Prof. Dr. Wolfgang Frisch, Universität Freiburg i. Br.

- Prof. Dr. Frank Höpfel, Universität Wien/Österreich und ad litem Richter beim ICTY, Den Haag/Niederlande

- Prof. Dr. Ursula Nelles, Universität Münster

- Prof. Dr. Mark Pieth, Universität Basel/Schweiz

- Prof. Dr. Klaus Volk, Universität München 


\section{DAS KURATORIUM}

Das Kuratorium des Instituts hat eine beratende und fördernde Funktion und ist mit hochrangigen Vertretern der Wissenschaft, Politik, Justiz und Justizverwaltung, Anwaltschaft und Medien besetzt. Seine Mitglieder werden vom Präsidenten der MPG auf Vorschlag des Instituts berufen. Das Kuratorium fördert die Beziehungen zwischen der Grundlagenforschung und verschiedenen gesellschaftlichen Bereichen sowie das Interesse der Öffentlichkeit an Forschungsergebnissen.

\section{Das Kuratorium setzt sich derzeit wie folgt zusammen:}

- Prof. Dr. Dr. h.c. mult. Winfried Hassemer, Ehemaliger Vizepräsident des Bundesverfassungsgerichts, Karlsruhe und Universität Frankfurt a.M. (Vorsitzender)

- Ministerialdirigent Achim Brauneisen, Justizministerium Stuttgart

- Franz Hermann Brüner, Generaldirektor des Europäischen Amtes für Betrugsbekämpfung (OLAF)

- Ministerialdirigent Thomas Dittmann, Bundesministerium der Justiz, Berlin

- Gernot Erler MdB, Staatsminister im Auswärtigen Amt, Berlin

- Peter Häberle, Leitender Oberstaatsanwalt, Freiburg i. Br.

- Monika Harms, Generalbundesanwältin, Karlsruhe

- Prof. Dr. Dr. Kristian Kühl, Universität Tübingen

- Dr. Heribert Prantl, Süddeutsche Zeitung, München

- Rechtsanwalt Prof. Dr. Franz Salditt, Neuwied

- Oberbürgermeister Dr. Dieter Salomon, Freiburg i. Br.

- Wolfgang Schomburg, Richter am ICTY (Berufungskammer), Den Haag/Niederlande

- Peter Wilkitzki, Ministerialdirektor a. D., Bonn

\section{Bis Anfang 2008 gehörten dem Kuratorium die folgenden Personen an:}

- Prof. Dr. Dr. h.c. mult. Winfried Hassemer, Ehemaliger Vizepräsident des Bundesverfassungsgerichts, Karlsruhe und Universität Frankfurt a.M. (Vorsitzender)

- Ministerialdirigent Herbert Bölter, Justizministerium Stuttgart

- Thomas Keller, Deutsche Bank, Freiburg i. Br.

- Prof. Dr. Dr. Kristian Kühl, Universität Tübingen

- Staatsministerin Kerstin Müller, Auswärtiges Amt, Berlin

- Generalbundesanwalt Kay Nehm, Karlsruhe

- Dr. Heribert Prantl, Süddeutsche Zeitung, München

- Rechtsanwalt Prof. Dr. Franz Salditt, Neuwied

- Oberbürgermeister Dr. Dieter Salomon, Freiburg i. Br.

- Dr. Wolfgang Schomburg, Richter am ICTY (Berufungskammer), Den Haag/Niederlande

- Peter Wilkitzki, Ministerialdirektor a.D., Bundesministerium der Justiz, Berlin 


\section{Personalien}


VI. PERSONALIEN

A. Ehrungen

B. Tätigkeit in wissenschaftlichen Organisationen

C. Selbstverwaltungsaktivitäten 


\section{A. Ehrungen}

Albrecht, Hans-Jörg

- Ernennung zum Gastprofessor an der Beijing Normal University, Peking (Mai 2006)

Eser, Albin

- Verleihung der Ehrennadel der Max-PlanckGesellschaft (Juni 2007)

\section{Lafrenz, Bianca}

- Preis des Vereins der Ehemaligen der Universität Konstanz (2006)

\section{Sieber, Ulrich}

- Ernennung zum Gastprofessor an der Beijing Normal University, Peking (Oktober 2007)

- Wahl zum Vizepräsidenten der „Association Internationale pour la Défence Sociale“ (September 2007)

\section{B. Tätigkeiten für wissenschaftliche Organisationen und Herausgeber- schaften}

\section{Albrecht, Hans-Jörg}

- Honorarprofessor und Fakultätsmitglied an der Albert-Ludwigs-Universität Freiburg

- Gastprofessor an der Juristischen Fakultät der Renmin-Universität/VR China

- UT- Professorship and Permanent Faculty Membership der Rechtswissenschaftlichen Fakultät des Qom High Education Center der Universität Teheran/Iran

- Gastprofessor an der Juristischen Fakultät der Universität Wuhan/VR China

- Mitherausgeber der Reihe „Kriminologische Forschungsberichte aus dem Max-Planck-Institut für ausländisches und internationales Strafrecht"

- Mitherausgeber der „Monatsschrift für Kriminologie und Strafrechtsreform“
- Mitherausgeber der Zeitschriften „Déviance et Société“, „European Journal of Crime, Criminal Law and Criminal Justice“ sowie „Recht der Jugend und des Bildungswesens"

- Mitherausgeber der Auslandsrundschau der Zeitschrift für die Gesamte Strafrechtswissenschaft (ZStW)

- Mitglied im Editorial Board der Zeitschrift „Journal on Terrorism and Organised Crime“ und dem Editorial Board und International Advisory Board der Zeitschrift „European Journal of Criminology"

- Mitglied im Advisory Board der Zeitschriften „Suchttherapie“, „International Journal of Policy and Practice“, „Police Practice and Research An International Journal“, „Studies on Crime and Crime Prevention“, „Revista de Derecho Penal y Criminología“ sowie der Zeitschrift „Crime \& Justice International“ 
- Mitglied des Beirats der Kriminologischen Zentralstelle e.V, des Instituts für interdisziplinäre Konflikt- und Gewaltforschung, Universität Bielefeld

- Kuratoriumsmitglied der Studienstiftung des Deutschen Volkes

- Mitglied im Scientific Advisory Board des bundesdeutschen Modellprojekts zur heroingestützten Behandlung Opiatabhängiger

- Mitglied des External Advisory Board of the Centre for Criminology, Universität of Oxford

- Mitglied des International Advisory Board des Schordijk Institute, Universität Tilburg, Niederlande

\section{Arnold, Jörg}

- Honorarprofessor an der Rechtswissenschaftlichen Fakultät der Westfälischen WilhelmsUniversität Münster

\section{Brunst, Phillip}

- Mitglied des Beirats der „Defence Aganist Terrorism Review"

\section{Eser, Albin}

• Tätigkeit als Richter am Internationalen Straftribunal für das frühere Jugoslawien (ICTY) in Den Haag bis Juni 2006

- Vorsitzender des Ehrengerichts der Deutschen Gesellschaft für Psychologie

- Mitglied des Stiftungsrates der ScheringStiftung Berlin

- Mitglied des Board of Directors der Association International de Droit Penal (Paris/Frankreich)

- Mitglied des Managing Committee der International Society for the Reform of Criminal Law (Vancouver/Kanada)

n Mitglied des Board of Advisers to the Institute of Criminology and Criminal Justice, Queen's University Belfast (Northern Ireland)

- Mitglied der Collaboradores Permanentes „Lex Medicinae Revista Portuguesa de Direito da Saúde" des Centro de Direito Biomédico

- Mitglied des Consejo Acesor der „Revista de Derecho Penal y Criminología“

- Mitglied des Advisory Board des „Maastricht Journal of European and Comparative Law“

- Mitglied des Advisory Board des Magazins „Derecho y Genoma Humano/Law and the Human
Genome Review" der Universidad de Deusto in Bilbao

- Mitglied des Wissenschaftlichen Beirats des „Jahrbuchs für Wissenschaft und Ethik“

- Mitglied des Consejo Consultivo Internacional der „Revista Peruana de Ciencias Penales“ (Buenos Aires/Argentinien)

- Mitglied des Academic Advisory Board der in Chicago/USA herausgegebenen Schriftenreihe „International and Comparative Criminal Law"

- Mitglied des Advisory Boards der von Oxford University Press herausgegebenen Publikation „The Rome Statute of the International Criminal Court: A Commentary“

n Mitherausgeber der Zeitschrift für die Gesamte Strafrechtswissenschaft (ZStW)

\section{Jescheck, Hans-Heinrich}

- Ehrenmitglied der Gesellschaft für Rechtsvergleichung

- Ehrenpräsident der Association Internationale de Droit Pénal

- Ausländisches Mitglied der Wissenschaftlichen Akademien der Niederlande, Norwegen, Finnland, Italien und des Großherzoglichen Instituts in Luxemburg

- Mitherausgeber der Zeitschrift für die Gesamte Strafrechtswissenschaft (ZStW)

\section{Kaiser, Günther $\dagger$}

- Mitglied des Wissenschaftlichen Beirats Kriminologisches Forschungsinstitut, Niedersachsen

- Mitglied des Beirats der Monatsschrift für Kriminologie und Strafrechtsreform

- Mitherausgeber des European Journal of Crime, Criminal Law and Criminal Justice

- Mitherausgeber der Reihe „Kriminologische Forschungsberichte aus dem Max-Planck-Institut für ausländisches und internationales Strafrecht" in der edition iuscrim

- Mitherausgeber der Zeitschrift für die Gesamte Strafrechtswissenschaft (ZStW)

\section{Kilchling, Michael}

- Deutscher Delegierter im Management Committee und Vorsitzender der Working Group 2 der COST Action A2 1 „Restorative Justice Developments in Europe“ der European Science Foundation

- Mitglied der Crime Proofing Steering Group 
der EU-Kommission, DG Justice and Home Affairs

- Mitglied im Vorstand des European Forum for Restorative Justice

- Mitglied der Group of Specialists on Assistance to Victims and Prevention of Victimisation (PC$\mathrm{S}-\mathrm{AV})$ des Europarats

- Gutachter für das European Journal on Criminal Policy and Research

- Mitglied der Informal Group of Experts on Confiscation and Asset Recovery bei der EU-Kommission, DG Justice, Freedom and Security

- Mitglied des Sellin-Glueck Award Committee 2007 der American Society of Criminology

- Mitglied der Steering Group zu dem AGIS-Projekt „Method and Assessment of Vulnerability of Sectors (MAVUS 1 und MAVUS 2)" des Institute for International Research on Criminal Policy (IRCP) der Universität Gent

- Mitglied des Wissenschaftlichen Beirats des Arbeitskreises der Opferhilfen in der Bundesrepublik Deutschland (ado)

\section{Koch, Hans-Georg}

- Vorsitzender der Lebendspende-Kommission ( 8 TPG) der Landesärztekammer BadenWürttemberg für den Bereich der Bezirksärztekammer Südbaden

- Berufung in den Arbeitskreis „Offene Fragen der Reproduktionsmedizin“ der Bundesärztekammer

- Mitglied der Ethik-Kommission der Universität Freiburg

- Mitglied des wissenschaftlichen Beirats der Zeitschrift „Ethik in der Medizin“

\section{Lukas, Tim}

- Mitglied des Koordinationsrates Kommunale Kriminalprävention der Stadt Freiburg

\section{Oberwittler, Dietrich}

- Affiliated Lecturer an der Law Faculty der University of Cambridge/Großbritannien für das akademische Jahr 2005/2006

\section{Quenzer, Carolin}

- Mitglied des forensischen Fachteams der Deutschen Psychologischen Akademie

\section{Sieber, Ulrich}

n Honorarprofessor und Fakultätsmitglied an der Ludwig Maximilians Universität München

- Honorarprofessor und Fakultätsmitglied an der Albert-Ludwigs-Universität Freiburg

- Gastprofessor an der Renmin Universität Peking

- Gastprofessor an der Beijing Normal University Peking

- Gastprofessor an der Universität Wuhan/China

- Vizepräsident der Association Internationale pour la Défence Sociale

n Mitglied im Conseil de Direction der Association Internationale de Droit Pénal (AIDP)

- Mitglied der „International Academy of Comparative Law"

- Ehrenmitglied der Japanischen Strafrechtslehrervereinigung

- German Contact Point for the European Criminal Law Academic Network (ECLAN)

- Präsident der Deutschen Vereinigung für Europäisches Strafrecht e.V.

- Vorstandsmitglied im Europäischen Rechtszentrum der Universität Würzburg

- Mitglied im Stiftungsrat der Deutschen Stiftung für Recht und Informatik (DSRI)

- Mitglied des Editorial Board der International Criminal Law Review

- Mitglied im Comité scientifique international der Zeitschrift „Revue de science criminelle et de droit pénal comparé" (RSC)

- Mitglied im „Correspondents Panel“ der Zeitschrift „The Computer Law and Security Report"

- Mitglied im Advisory Board der Zeitschrift „Money Laundering Control“

n Mitglied im Consultative International Council of the „Revista Penal“

- Mitglied im Beirat der Online-Zeitschrift „European Review for ICT-Law"

- Mitherausgeber der Auslandsrundschau der Zeitschrift für die gesamte Strafrechtswissenschaft (ZStW)

- Mitherausgeber der Zeitschrift „Multimedia und Recht"

- Mitglied im Herausgeberbeirat der Zeitschrift „Computer und Recht“

- Mitherausgeber des „European Journal of Crime, Criminal Law and Criminal Justice“

- Herausgeber der online-Zeitschrift „eucrim“ 
- Herausgeber der Reihe „Strafrechtliche Forschungsberichte" aus dem Max-Planck-Institut für ausländisches und internationales Strafrecht

- Mitherausgeber der Reihe „Sammlung ausländischer Strafgesetzbücher" aus dem Max-PlanckInstitut für ausländisches und internationales Strafrecht

- Herausgeber der europäischen Schriftenreihen „ius informationis“ und „ius criminale“

- Mitherausgeber der Buchreihe „ius europeum“

- Mitherausgeber „Handbuch Multimedia Recht“

\section{Simon, Jan-Michael}

- Mitglied des Research Council des Eurowarrant Projekts, T.M.C. Asser Institute, Den Haag

- Mitglied der Working Group 4, COST Action 21

- Restorative Justice Developments in Europe

\section{Tellenbach, Silvia}

- Mitglied des Vorstands der Gesellschaft für arabisches und islamisches Recht e.V.

\section{Wade, Marianne}

- Fellow der Gottlieb-Daimler- und Karl-BenzStiftung

- Mitglied der „International Advisory Group Member“ für das „Programme on Strengthening the Rule of Law in the Arab States - Modernization of Public Prosecution Offices“, innerhalb des „Programme on Governance in the Arab Region“ („POGAR) des United Nations Development Programme (UNDP)

च Mitglied des „Legal Experts Advisory Panel“ für Fair Trials International („FTI“)

- Gastherausgeberin des European Journal on Criminal Policy and Research „Fear v. Freedom Post 9/11 - A European Perspective“ 


\section{Selbstverwaltungsaktivitäten}

\section{MAX-PLANCK-GESELLSCHAFT}

\section{Albrecht, Hans-Jörg}

- Mitglied der Geistes-, Sozial- und Humanwissenschaftlichen Sektion der Max-Planck-Gesellschaft

- Schlichtungsberater der Geistes-, Sozial- und Humanwissenschaftlichen Sektion

- Mitglied der Arbeitsgruppe „Zusammenarbeit der juristischen Institute in der MPG mit den Wirtschaftswissenschaften und anderen sozialwissenschaftlichen Disziplinen“

\section{Arnold, Jörg}

- Mitglied der MPG-Berufungskommission für das Max-Planck-Institut für ausländisches und internationales Sozialrecht in München

- Mitglied der MPG-Berufungskommission für das Max-Planck-Institut für ausländisches und öffentliches Recht und Völkerrecht in Heidelberg

\section{Brunst, Phillip}

- Leitung der Projektgruppe „Content-Management" von fünf juristischen Max-Planck-Instituten

\section{Cornils, Karin}

- Mitglied im Arbeitskreis der Max-Planck-Gesellschaft zur „Förderung der Wissenschaftlerinnen"
Eser, Albin

n Mitglied der Ständigen Präsidentenkommission „Ethikrat“ der Max-Planck-Gesellschaft

\section{Koch, Hans-Georg}

- Gast der Arbeitsgruppe „Sicherheits- und Verteidigungsforschung" der Max-Planck-Gesellschaft (Dezember 2006, Berlin)

\section{Sieber, Ulrich}

- Sektionsberater der Geistes-, Sozial- und Humanwissenschaftlichen Sektion der MaxPlanck-Gesellschaft zur Verleihung der OttoHahn-Medaillen

- Mitglied der Perspektivenkommission der Geistes-, Sozial- und Humanwissenschaftlichen Sektion der Max-Planck-Gesellschaft

- Vorsitzender der Arbeitsgruppe des Wissenschaftlichen Rats über „Sicherheits- und Verteidigungsforschung"

n Mitglied der Ständigen Präsidentenkommission „Ethikrat“ der Max-Planck-Gesellschaft

- Vorsitzender der MPG-Berufungskommission für das Max-Planck-Institut für ethnologische Forschung in Halle

- Mitglied der Berufungskommission für das Max-Planck-Institut für ausländisches und internationales Sozialrecht in München 


\section{MAX-PLANGK-INSTITUT FÜR AUSLÄNDISCHES UND INTERNATIONALES STRAFRECHT}

\section{Arnold, Jörg}

- Ombudsperson des Max-Planck-Instituts für ausländisches und internationales Strafrecht zur Sicherung guter wissenschaftlicher Praxis

- Vertreter der wissenschaftlichen Mitarbeiter und Mitarbeiterinnen des Max-Planck-Instituts für ausländisches und internationales Strafrecht in der Geistes-, Human- und Sozialwissenschaftlichen Sektion der Max-PlanckGesellschaft

\section{Cornils, Karin}

- Frauenbeauftragte

- Gleichstellungsbeauftragte

Engelhart, Marc

- Doktorandensprecher

\section{Grundies, Volker}

n Datenschutzverantwortlicher

Holzmann, Bernhard

[ Vorsitzender des Betriebsrats

\section{Holzmann, Elfriede}

- Schwerbehindertenvertreterin

Koch, Hans-Georg

- Stellvertretender Datenschutzverantwortlicher

Macke, Julia

- Doktorandensprecherin

Quenzer, Carolin

- Doktorandensprecherin

\section{Reinhard, Simone}

- Jugend- und Auszubildendenvertreterin

\section{Rinceanu, Johanna}

- Stellvertretende Frauenbeauftragte

- Stellvertretende Gleichstellungsbeauftragte

Spoenle, Jan

- Doktorandensprecher 
Anhang 
ANHANG

143

159

172

174

176

A. Publikationen

B. Vorträge

C. Veranstaltungen

D. Lehre

E. Doktoranden 


\section{A. Publikationen}

\section{Abdelbaqi, Mustafa H. 2006}

Abdelbaqi, M.H.: Introduction to the Palestinian Criminal Justice System. forschung aktuell $\mid$ research in brief 35. edition iuscrim. Freiburg i. Br. 2006. (56 S).

\section{Albrecht, Hans-Jörg 2006}

Albrecht, H.-J.: Counterterrorism Policies in Germany. forschung aktuell $\mid$ research in brief 38. edition iuscrim. Freiburg i. Br. 2006. (69 S.).

Albrecht, H.-J.: Stalking - Nationale und Internationale Rechtspolitik und Gesetzesentwicklung. Familie Partnerschaft Recht 12, 204-208 (2006).

Albrecht, H.-J.: Terrorismus und Strafrecht. In: Griesbaum, R., Hannich, R., Schnarr, K.H. (Hrsg.), Strafrecht und Justizgewährung. Festschrift für Kay Nehm zum 65. Geburtstag. Berliner Wissenschafts-Verlag. Berlin 2006, S. 17-26.

Albrecht, H.-J.: Illegalität, Kriminalität und Sicherheit. In: Alt, J., Bommes, M. (Hrsg.), Illegalität. Grenzen und Möglichkeiten der Migrationspolitik. VS Verlag für Sozialwissenschaften. Wiesbaden 2006, S. 60-80.

Albrecht, H.-J.: Antworten auf Gefährlichkeit - Sicherungsverwahrung und unbestimmter Freiheitsentzug. In: Feltes, Th., Pfeiffer, Ch., Steinhilper, G. (Hrsg.), Kriminalpolitik und ihre wissenschaftlichen Grundlagen: Festschrift für Prof. Dr. Hans-Dieter Schwind zum 70. Geburtstag. C. F. Müller Verlag. Heidelberg 2006, S. 191-210.

Albrecht, H.-J.: Forensik - DNA-Analyse - Recht, Praxis und Entwicklungen. In: Labor \& More 3, 28-29 (2006).

Albrecht, H.-J.: Fußball und Gewalt. Entwicklungen, Erklärungsansätze und Prävention. In: Monatsschrift für Kriminologie und Strafrechtsreform 89, 3, 158-174 (2006).

Albrecht, H.-J.: Elektronische Überwachung in Europa. In: Saimeh, N. (Hrsg.), Gesellschaft mit beschränkter Haftung. Maßregelvollzug als soziale Verpflichtung. Forensik 2006. 21. Eickelborner Fachtagung zu Fragen der Forensischen Psychiatrie, 1. bis 3. März 2006. Psychiatrie-Verlag. Bonn 2006, S. 13-33.

Albrecht, H.-J.: Regaining Trust and Confidence in Post-Conflict Societies as a Way to Prevent Terrorism. In: Ewald, U., Turković, K. (Hrsg.), Large-Scale Victimisation as a Potential Source of Terrorist Activities. Importance of Regaining Security in Post-Conflict Societies. NATO Security through Science Series. IOS Press. Amsterdam 2006, S. 30-53.

Albrecht, H.-J.: Conflict Perspectives: Dealing with Wrongs in the Middle East. In: Albrecht, H.-J., Simon, J.-M., Rezaei, H., Rohne, H.-C., Kiza, E. (Hrsg.), Conflicts and Conflict Resolution in Middle Eastern Societies - Between Tradition and Modernity. Schriftenreihe des MaxPlanck-Instituts für ausländisches und internationales Strafrecht. Interdisziplinäre Forschungsberichte aus Strafrecht und Kriminologie I 13. Duncker \& Humblot. Berlin 2006, S. 1-12.
Albrecht, H.-J.: Trafficking in Humans. The Phenomenon, Theory and Criminal Law Based Responses. In: Fenyvesi, C., Herke, C., Mészáros, B. (Hrsg.), Bizonyítékok. Tiszteletkötet Tremmel Flórián Egyetemi Tanár 65. Születésnapjára. Tudományegyetem Állam- és Jogtudományi Kara. Pécs 2006, S. 9-29.

Albrecht, H.-J.: Le système de sanctions et de condamnations pénales de la République fédérale allemande. In: Poncela, P., Roth, R. (Hrsg.), La fabrique du droit des sanctions pénales au Conseil de l'Europe. Mission de Recherche, Droit et Justice, La Documentation Française. Paris 2006, S. 81-117.

Albrecht, H.-J.: Corruption and Corruption Control. In: Chen, Z. (Hrsg.), New Reports in Criminal Law. Chinese People's Public Security University Press. Beijing 2006, S. 331-352.

Albrecht, H.-J.: Analysis Report on Status of Defence Counsel. In: Chen, Z. (Hrsg.), Strengthening the Defence in Death Penalty Cases. Forum of Criminal Law Department of CASS - Chinese Academy of Social Sciences. Beijing 2006, S. 69-132.

Albrecht, H.-J.: Extranjería, migración, inmigración y evolución de la justicia penal en Europa. In: Editores del Puerto (Hrsg.), NDP. Nueva Doctrina Penal. 2006/A. Edición argentina. Buenos Aires 2006, S. 3-28.

Albrecht, H.-J.: Sistemas de sanciones penales: presente y futuro. In: Instituto Nacional de Ciencias Penales (INACIPE), Max-Planck-Institut für ausländisches und internationales Strafrecht (Hrsg.), Hacia la unificación del derecho penal. Logros y desafíos de la armonización y homologación legislativa en México y en el mundo. INACIPE. México 2006, S. 803-851.

Albrecht, H.-J.: A biztonságkoncepció átalakulása és ennek következményei az európai bel- és jogpolitkára. In: Korinek, L. (Hrsg.), Belügyi Szemle - A Belügyminisztérium Szakmai, Tudományos Folyóirata. Innenministerium Ungarn. Budapest 2006, S. 3-26.

Albrecht, H.-J.: Imprisonment and Alternatives to Prisons: Changes and Prospects in a Comparative Perspective. In: Revista Académica - Facultad de Derecho de la Universidad la Salle III, 6, 27-50 (2006).

Albrecht, H.-J.: Kriminologische Forschung am MaxPlanck-Institut für ausländisches und internationales Strafrecht Freiburg. In: Höfer, S., Spieß, G. (Hrsg.), Neuere Kriminologische Forschung im Südwesten. Eine Darstellung der Forschungsarbeit aus Anlass des 40. Kolloquiums der Südwestdeutschen und benachbarten Kriminologischen Institute. edition iuscrim. Freiburg i. Br. 2006, S. 67-98.

Albrecht, H.-J.: Respuestas legislativas al 11 de septiembre. Un análisis comparado de la legislación antiterrorista. In: Guzmán Dálbora, J. L., Serrano Maíllo, A. (Hrsg.), Derecho penal y criminología como fundamento de la política criminal - Estudios en homenaje al Profesor Alfonso Serrano Gómez. Dykinson. Madrid 2006, S. 1139-1164.

Albrecht, H.-J., Kania, H., Walter, M.: Einführung zu: Alltagsvorstellungen von Kriminalität. In: Walter, M., Neubacher, F. (Hrsg.), Neue Wege und Perspektiven der Kriminologie. Forschung am Institut für Kriminologie der Universität zu Köln. Kölner Schriften zur Kriminologie und Kriminalpolitik 12. LIT-Verlag. Berlin 2006, S. 291-316. 
Albrecht, H.-J., Kilchling, M.: Victims of Terrorism Policies and Legislation in Europe: an Overview on VictimRelated Assistance and Support (Expert Report). In: Council of Europe (Hrsg.), Victims - Support and assistance. Council of Europe Publishing. Strasbourg 2006, S. 199-250.

Albrecht, H.-J., Kilchling M.: Institutions and organisations responsible for victims (Contacts). In: Council of Europe (Hrsg.), Victims - Support and assistance. Council of Europe Publishing. Strasbourg 2006, S. 253-262.

Albrecht, H.-J., Kilchling, M.: Victimes du terrorisme - Politiques et législations européennes: Tour d'horizon des systèmes d'assistance aux victimes (Rapport d'expertise). In: Conseil de l'Europe (Hrsg.), Soutien et aide aux victimes. Les Éditions du Conseil de l'Europe. Strasbourg 2006 S. 209-266.

Albrecht, H.-J., Kilchling, M.: Institutions et organisations responsables pour les victimes [Contacts]. In: Consei de l'Europe (Hrsg.), Soutien et aide aux victimes. Les Éditions du Conseil de l'Europe. Strasbourg 2006, S. 269-278.

Albrecht, H.-J. and Research Unit of the Death Penalty Cases Survey Institute of Law: Strengthening the Defence in Death Penalty Cases in the People's Republic of China Empirical Research into the Role of Defence Councils in Criminal Cases Eligible for the Death Penalty. forschung aktuell | research in brief 37. edition iuscrim. Freiburg i. Br. 2006 (152 S.)

Albrecht, H.-J., Sieber, U. (Hrsg.): Strafrecht und Kriminologie unter einem Dach. Kolloquium zum 90. Geburtstag von Professor Dr. Hans-Heinrich Jescheck am 10. Januar 2005. Schriftenreihe des Max-Planck-Instituts für ausländisches und internationales Strafrecht. Interdisziplinäre Forschungsberichte aus Strafrecht und Kriminologie I 14. Duncker \& Humblot. Berlin 2006 (202 S.)

Albrecht, H.-J., Sieber, U. (Hrsg.): Perspektiven de strafrechtlichen Forschung. Amtswechsel am Freiburge Max-Planck-Institut für ausländisches und internationales Strafrecht 2004. Schriftenreihe des Max-Planck-Institut für ausländisches und internationales Strafrecht. Interdisziplinäre Forschungsberichte aus Strafrecht und Kriminologie I 12. Duncker \& Humblot. Berlin 2006 (79 S.).

Albrecht, H.-J., Simon, J.-M., Rezaei, H., Rohne, H.-C., Kiza, E. (Hrsg.): Conflicts and Conflict Resolution in Middle Eastern Societies - Between Tradition and Modernity. Schriftenreihe des Max-Planck-Instituts für ausländisches und internationales Strafrecht. Interdisziplinäre Forschungsberichte aus Strafrecht und Kriminologie I 13 Duncker \& Humblot. Berlin 2006 (660 S.).

Albrecht, H.-J., Walter, M., Kania, H.: Schlussbetrachtungen zu: Alltagsvorstellungen von Kriminalität. In: Walter M., Neubacher, F. (Hrsg.), Neue Wege und Perspektiven de Kriminologie. Forschung am Institut für Kriminologie de Universität zu Köln. Kölner Schriften zur Kriminologie und Kriminalpolitik 12. LIT-Verlag. Berlin 2006, S. 317-322.

\section{7}

Albrecht, H.-J.: Rechtliche Grundlagen der Forensischen Psychiatrie - eine international vergleichende Perspektive. In: Kröber, H.-L., Dölling, D., Leygraf, N., Sass, H (Hrsg.), Handbuch der Forensischen Psychiatrie. Steinkopff Verlag. Heidelberg 2007, S. 511-573.

Albrecht, H.-J.: Jugendfreiheitsstrafe und Jugendstrafvollzug im europäischen Ausland. In: Recht der Jugend und des Bildungswesens 55, 2, 201-211 (2007).

Albrecht, H.-J.: Straffälligenhilfe, Kosten-Nutzen-Analyse und strafrechtliche Kriminalitätsprävention. In: Schöch, H., Helgerth, R., Dölling, D., König, P. (Hrsg.), Festschrift für Reinhard Böttcher zum 70. Geburtstag am 29. Juli 2007. De Gruyter Recht. Berlin 2007, S. 235-261

Albrecht, H.-J.: Internationale Kriminalität, Gewaltökonomie und Menschenrechtsverbrechen: 10 Antworten des Strafrechts. In: Internationale Politik und Gesellschaft - International Politics and Society, 2, 153-169 (2007).

Albrecht, H.-J.: Forensische Sachverständige im Strafverfahren - Entwicklungen aus einer komparativen Perspektive. In: Kaiafa-Gbandi, M. et al. (Hrsg.), Festschrift für Ioannis Manoledakis. Sakkoulas. Athen et al. 2007, S. $711-727$.

Albrecht, H.-J.: Perspektiven kriminologischer Forschung. Der Wandel im Konzept der Sicherheit und neue Aufgabenfelder der Kriminologie. In: Liebl, K. (Hrsg.), Kriminologie im 21. Jahrhundert. VS Verlag für Sozialwissenschaften. Wiesbaden 2007, S. 177-201.

Albrecht, H.-J.: Vergleichende Kriminologie. In Schneider, H. J. (Hrsg.), Internationales Handbuch der Kri minologie. De Gruyter Recht. Berlin 2007, S. 255-288.

Albrecht, H.-J.: Legitimacy and Criminal Justice: Inequality and Discrimination in the German CriminalJustice System. In: Tyler, T.R. (Hrsg.), Legitimacy and Criminal Justice. Russell Sage Foundation. New York 2007 S. 302-332.

Albrecht, H.-J.: Trafficking in Humans and Human Rights. In: Parmentier, S., Weitekamp, E. G., Elsevier, M (Hrsg.), Crime and Human Rights. JAI Press, Amsterdam et al. 2007, S. 39-71.

Albrecht, H.-J.: The Rule of Law and Protection of the Personal Right. In: Wang, J. (Hrsg.), Personal Right and Rule of Law. China Forum on the Rule of Law Series. Social Sciences Academic Press (China). Beijing 2007, S. 28-38.

Albrecht, H.-J.: International Organized Crime: Reaction in Germany. In: Ueda, K. (Hrsg.), Current International Organized Crime: Global and in Japan. Human Security and Transnational Organized Crime. Nippon-Hyoron-Sha. Tokyo 2007, S. 194-224.

Albrecht, H.-J.: Kriminologische Forschung am MaxPlanck-Institut für ausländisches und internationales Straf recht Freiburg. In: Höfer, S. Spieß, G. (Hrsg.), Neuere Kriminologische Forschung im Südwesten. Eine Darstellung der Forschungsarbeit aus Anlass des 40. Kolloquiums der Südwestdeutschen und benachbarten Kriminologischen Institute: 2. akt. Aufl. edition iuscrim, Freiburg i. Br. 2007 S. 67-98.

Albrecht, H.-J., Grundies, V.: Sexuelle Gewaltkriminalität im Lebenslängsschnitt. Die Entwicklung von Sexualkriminalität an Hand von Daten der Freiburger Kohortenstudie. In Lösel, F., Bender, D., Jehle, J.-M. (Hrsg.), Kriminologie und wissensbasierte Kriminalpolitik. Entwicklungs- und Evaluationsforschung. Neue Kriminologische Schriftenreihe. Forum Verlag Godesberg. Mönchengladbach 2007, S. 447-475.

Albrecht, H.-J., Kilchling, M.: Victims of Terrorism Policies - Should Victims of Terrorism be Treated Differently? In: European Journal on Criminal Policy and Research 13, $1-2,13-31(2007)$

Albrecht, H.-J., Klemencic, G. (Contributors), O'Connor, V., Rausch C. (Hrsg.): Model Codes for Post-Conflict Criminal Justice. United States Institute of Peace Press. Washington 2007. (440 S.).

Albrecht, H.-J., Sieber, U.: Stellungnahme zu dem Fragenkatalog des Bundesverfassungsgerichts in dem Verfah ren 2 BvR 392/07 zu \$173 Abs. 2 S. 2 StGB - Beischlaf zwischen Geschwistern. 2007 (132 S.). http://www.mpicc. de/de/data/pdf/05-08-inzest_gutachten.pdf

\section{Arnold, Jörg}

2006

Arnold, J.: Entwicklungslinien des Feindstrafrechts in 5 Thesen. In: Höchstrichterliche Rechtsprechung im Strafrecht, 8-9, 303-315 (2006). 
Arnold, J.: Zum Geleit: Ende der Gespensterjagd und Beginn der wissenschaftlichen Debatte. In: Organisationsbüro. Schriftenreihe der Strafverteidigervereinigungen (Hrsg.), Bitte bewahren Sie Ruhe. Leben im Feindrechtsstaat. Eigenverlag der Strafverteidigervereinigungen. Berlin 2006, S. 13-25.

Arnold, J.: Criminal Law as a Reaction to System Crime: Policy for Dealing with the Past in European Transitions. In: Borejsza, J.W., Ziemer, K. (Hrsg.), Totalitarian and Authoritarian Regimes in Europe. Legacies and Lesson from the Twentieth Century. Berghan Books. New York 2006, S. 399-430.

Arnold, J., Eser, A.: Einführung in das Gesamtprojekt. In: Eser, A., Sieber, U., Arnold, J. (Hrsg.), Strafrecht in Reaktion auf Systemunrecht. Vergleichende Einblicke in Transitionsprozesse. Teilband 9: China (Richter). Schriftenreihe des Max-Planck-Instituts für ausländisches und internationales Strafrecht. Strafrechtliche Forschungsberichte S 82.9. Duncker \& Humblot. Berlin 2006, S. 1-22.

Arnold, J., Eser, A.: Einführung in das Gesamtprojekt. In: Eser, A., Sieber, U., Arnold, J. (Hrsg.), Strafrecht in Reaktion auf Systemunrecht. Vergleichende Einblicke in Transitionsprozesse. Teilband 10: Südkorea (Cho/Holzapfl/ Richter). Schriftenreihe des Max-Planck-Instituts für ausländisches und internationales Strafrecht. Strafrechtliche Forschungsberichte S 82.10. Duncker \& Humblot. Berlin 2006, S. 1-22.

Arnold, J., Eser, A.: Prólogo. In: Aponte, A. (Hrsg.), Guerra y derecho penal de enemigo. Reflexión crítica sobre el eficientismo penal de enemigo. Grupo Editorial IBAÑEZ. Bogotá 2006, S. 15-20.

Arnold, J., Eser, A., Sieber, U. (Hrsg.): Strafrecht in Reaktion auf Systemunrecht. Vergleichende Einblicke in Transitionsprozesse. Teilband 9: China (Richter). Schriftenreihe des Max-Planck-Instituts für ausländisches und internationales Strafrecht. Strafrechtliche Forschungsberichte S 82.9. Duncker \& Humblot. Berlin 2006 (267 S.).

Arnold, J., Eser, A., Sieber, U. (Hrsg.): Strafrecht in Reaktion auf Systemunrecht. Vergleichende Einblicke in Transitionsprozesse. Teilband 10: Südkorea (Cho/Holzapfl/ Richter). Schriftenreihe des Max-Planck-Instituts für ausländisches und internationales Strafrecht. Strafrechtliche Forschungsberichte S 82.10. Duncker \& Humblot. Berlin 2006 (126 S.).

Arnold, J., Simon, J.-M., Woischnik, J. (Hrsg.): Estado de Derecho y Delincuencia de Estado en América Latina. Una visión comparativa. Universidad Nacional Autónoma de México. México 2006 (420 S.).

\section{7}

Arnold, J.: Protection of Human Rights by Means of Criminal Law: On the Relationship between Criminal Law and Politics. In: Kaleck, W., Ratner, M., Singelnstein, T., Weiss, P. (Hrsg.), International Prosecution of Human Rights Crimes. Springer. Heidelberg 2007, S. 3-12.

Arnold, J., Eser, A.: Einführung in das Gesamtprojekt. In: Eser, A., Sieber, U., Arnold, J. (Hrsg.), Strafrecht in Reaktion auf Systemunrecht. Vergleichende Einblicke in Transitionsprozesse. Teilband 11: Chile (Millaleo Hernández), Uruguay (Fernández). Schriftenreihe des Max-PlanckInstituts für ausländisches und internationales Strafrecht. Strafrechtliche Forschungsberichte S 82.11. Duncker \& Humblot. Berlin 2007, S. 1-16.

Arnold, J., Eser, A., Sieber, U. (Hrsg.): Strafrecht in Reaktion auf Systemunrecht. Vergleichende Einblicke in Transitionsprozesse. Teilband 11: Chile (Salvador A. Millaleo Hernández), Uruguay (Gonzalo D. Fernández). Schriftenreihe des Max-Planck-Instituts für ausländisches und internationales Strafrecht. Strafrechtliche Forschungsberichte S 82.11. Duncker \& Humblot. Berlin 2007 (643 S.)

\section{Aydin, Öykü Didem 2006}

Aydin, Ö.D.: Die strafrechtliche Bekämpfung von Hassdelikten in Deutschland und in den Vereinigten Staaten von Amerika. Ein Vergleich des kriminologischen, strafrechtstheoretischen und verfassungsrechtlichen Umgangs beider Systeme mit der Fremdenfeindlichkeit unter besonderer Berücksichtigung der Vorschrift der Volksverhetzung - $\$ 130$ des deutschen StGB - und der U.S.-Amerikanischen Gesamtproblematik von Hate Speech/Crimes. Schriftenreihe des Max-Planck-Instituts für ausländisches und internationales Strafrecht. Kriminologische Forschungsberichte K 125. Freiburg i.Br. 2006 (520 S.).

\section{Bellini, Marcello 2006}

Bellini, M.: Portknocking - Zugang erst nach Anklopfen. In: Gartmann, C., Jähnke, J. (Hrsg.), 22. DV-Treffen der Max-Planck-Institute. 16.-18. November 2005 in Göttingen. GWDG-Berichte 70. Gesellschaft für wissenschaftliche Datenverarbeitung mbH Göttingen. Göttingen 2007, S. 69-76.

\section{Brandenstein, Martin} 2006

Brandenstein, M.: Strafzweckerfüllungen als abhängige Variable der Zeit. In: Obergfell-Fuchs, J., Brandenstein, M (Hrsg.), Nationale und internationale Entwicklungen in der Kriminologie - Festschrift für Helmut Kury zum 65. Geburtstag. Verlag für Polizeiwissenschaft. Frankfurt a.M. 2006, S. 357-394.

Brandenstein, M., Kury, H.: Wahrnehmung und (Rechts-)Wirklichkeit der Verkehrsdelinquenz. In: Zeitschrift für Verkehrssicherheit 52, 1, 7-12 (2006).

Brandenstein, M., Obergfell-Fuchs, J.: Helmut Kury zum 65. Geburtstag. In: Obergfell-Fuchs, J., Brandenstein, M. (Hrsg.), Nationale und internationale Entwicklungen in der Kriminologie - Festschrift für Helmut Kury zum 65. Geburtstag. Verlag für Polizeiwissenschaft. Frankfurt a.M. 2006, S. 11-16.

Brandenstein, M., Obergfell-Fuchs, J. (Hrsg.): Nationale und internationale Entwicklungen in der Kriminologie - Festschrift für Helmut Kury zum 65. Geburtstag. Verlag für Polizeiwissenschaft. Frankfurt a.M. 2006 (687 S.)

\section{7}

Brandenstein, M., Özsöz, F.: Hasskriminalität: Auswirkungen von Hafterfahrungen auf fremdenfeindliche jugendliche Gewalttäter. In: Max-Planck-Gesellschaft zur Förderung der Wissenschaften e. V. (Hrsg.), Jahrbuch 2007 der Max-Planck-Gesellschaft - CD-ROM-Anlage. MaxPlanck-Gesellschaft zur Förderung der Wissenschaften e. V. München 2007 (5 S.).

\section{Brunst, Phillip W.}

\section{7}

Brunst, P.W., Sieber, U.: Cyberterrorism and other use of the Internet for terrorist purposes. Threat analysis and evaluation of international conventions. In: Council of Europe (Hrsg.), Cyberterrorism and other use of the Internet for terrorist purposes. Straßburg, 2007, S. 10-105. 


\section{Cornils, Karin}

2006

Cornils, K., Frände D., Matikkala J.: Das finnische Strafgesetz - Rikoslaki - Strafflag, nach dem Stand vom 1.10.2005. Übersetzung und Einführung von Karin Cornils, Dan Frände und Jussi Matikkala. Dreisprachige Ausgabe. Schriftenreihe des Max-Planck-Instituts für ausländisches und internationales Strafrecht. Sammlung ausländischer Strafgesetzbücher in deutscher Übersetzung G 116. Duncker \& Humblot. Berlin 2006 (563 S.).

Cornils, K., Greve V.: Landesbericht Dänemark. In Gropp, W., Sinn, A. (Hrsg.), Organisierte Kriminalität und kriminelle Organisationen. Präventive und repressive Maßnahmen vor dem Hintergrund des 11 . September 2001. Nomos. Baden-Baden 2006, S. 32-71.

\section{7}

Cornils, K.: National Criminalization of International Crimes - Comparative Aspects. In: Nuotio, K. (Hrsg.), Fest schrift in Honour of Raimo Lahti, Forum Iuris. University of Helsinki. Helsinki 2007, S. 25-42.

\section{Diniz Pucci, Rafael} 2006

Diniz Pucci, R.: Brazil on Trial: Mafia, Organized Crime, Gang, Terrorist Group - or, Simply, a Problem Created by a State Policy? forschung aktuell | research in brief 36. edition iuscrim. Freiburg i. Br. 2006 (24 S.)

\section{Ehrmann, Jens}

\section{7}

Ehrmann, J.: Die belebte Innenstadt als Rechtsproblem. Zum rechtlichen Instrumentarium zur Erhaltung funktionsfähiger städtischer Zentren. Schriften zum deutschen und europäischen Kommunalrecht 28. Boorberg. Stuttgart 2007 (216 S.).

\section{Engelhart, Marc \\ 2006}

Engelhart, M., Zhou, Y.: Case Materials on Judicial Methodology in Criminal Law. In: GTZ (Hrsg.), Course Materials on Judicial Methodology. Peking 2006.

\section{Eser, Albin}

2006

Eser, A.: Perspektiven des Medizin(straf)rechts. In Frisch, Wolfgang (Hrsg.), Gegenwartsfragen des Medizinstrafrechts. Portugiesisch-deutsches Symposium zu Ehren von Albin Eser in Coimbra. Ethik und Recht in der Medizin 41. Nomos. Baden-Baden 2006, S. 9-31.

Eser, A.: Einführung, 1-10, Vorbemerkung zu § 1, $11-23$, Nullum Crimen sine lege - Wahlfeststellung (\$\$ 1, 2), 23-69, Geltungsbereich - Internationales Strafrecht (\$\$3-12) 69-147, Versuch und Rücktritt ( $\$ \$ 22-24), 420-482$, Verfal und Einziehung ( $\$ \$ 73-76 a), 1022-1070$, Straftaten gegen ausländische Staaten ( $\$ \$ 102-104 a), 1180-1183$, Straftaten gegen Verfassungsorgane ( $\$ \$ 105-108 \mathrm{e}), 1183-1194$, Straftaten gegen die Landesverteidigung ( $\$ \$ 109-109 \mathrm{k}), 1194-1209$ Widerstand gegen die Staatsgewalt (\$\$ 110-121), 1209-1238, Straftaten gegen das Leben (\$211-222), 1768-1901, Körper- verletzung - Ärztliche Heilbehandlung (\$223), 1901-1933, Straftaten gegen die persönliche Freiheit (\$\$ 234-24la), 1980-2036, Diebstahl und Unterschlagung (\$\$242-249), 2037-2099, Raub und Erpressung (\$\$249-256), 2099-2122, In: Schönke, A., Schröder, H., Eser, A., et al., Strafgesetzbuch, Kommentar. 27. Aufl. Verlag C.H. Beck. München 2006.

Eser, A.: Justizielle Rechte. In: Meyer, J. (Hrsg.), Kommentar zur Charta der Grundrechte der Europäischen Union. Nomos. Baden-Baden 2006, S. 477-526.

Eser, A.: Zur Schlüsselrolle des Anklägers für die internationale Strafjustiz. In: Griesbaum, R., Hannich, R., Schnarr, K.H. (Hrsg.), Strafrecht und Justizgewährung. Festschrift für Kay Nehm zum 65. Geburtstag. Berliner Wissenschafts-Verlag. Berlin 2006, S. 111-124.

Eser, A.: Gedanken im Übergang. In: Albrecht, H.-J., Sieber, U. (Hrsg.), Perspektiven der strafrechtlichen Forschung. Amtswechsel am Freiburger Max-Planck-Institut für ausländisches und internationales Strafrecht 2004, Schriftenreihe des Max-Planck-Instituts für ausländisches und internationales Strafrecht. Interdisziplinäre Forschungsberichte aus Strafrecht und Kriminologie I 12. Duncker \& Humblot. Berlin 2006, S. 21-34.

Eser, A.: Das Internationale Militärtribunal von Nürnberg aus deutscher Perspektive. In: Reginbogin, H. R., Safferling, C. J. M. (Hrsg.), Die Nürnberger Prozesse. Völkerstrafrecht seit 1945. K. G. Saur. München 2006, S. 53-59.

Eser, A.: Festvortrag 2002: Wahrheit und Wahrhaftigkeit in der Wissenschaft. In: Meister, W. (Hrsg.), GlaxoSmithKline Stiftung. Die Jahre seit 1978. GlaxoSmithKline Stiftung. München 2006, S. 66-73.

Eser, A.: Nachwort des Projektinitiators. In: Eser, A., Sieber, U., Kreicker, H. (Hrsg.), Nationale Strafverfolgung völkerrechtlicher Verbrechen. National Prosecution of International Crimes. Teilband 7: Völkerstrafrecht im Ländervergleich (Kreicker). Schriftenreihe des Max-PlanckInstituts für ausländisches und internationales Strafrecht. Strafrechtliche Forschungsberichte S 95.7. Duncker \& Humblot. Berlin 2006, S. 419-422.

Eser, A., Arnold, J.: Einführung in das Gesamtprojekt. In: Eser, A., Sieber, U., Arnold, J. (Hrsg.), Strafrecht in Reaktion auf Systemunrecht. Vergleichende Einblicke in Transitionsprozesse. Teilband 9: China (Richter). Schriftenreihe des Max-Planck-Instituts für ausländisches und internationales Strafrecht. Strafrechtliche Forschungsberichte S 82.9. Duncker \& Humblot. Berlin 2006, S. 1-22.

Eser, A., Arnold, J.: Einführung in das Gesamtprojekt. In: Eser, A., Sieber, U., Arnold, J. (Hrsg.), Strafrecht in Reaktion auf Systemunrecht. Vergleichende Einblicke in Transitionsprozesse. Teilband 10: Südkorea (Cho/Holzapfl/ Richter). Schriftenreihe des Max-Planck-Instituts für ausländisches und internationales Strafrecht. Strafrechtliche Forschungsberichte S 82.10. Duncker \& Humblot. Berlin 2006, S. 1-22.

Eser, A., Arnold, J.: Prólogo. In: Aponte, A. (Hrsg.), Guerra y derecho penal de enemigo. Reflexión crítica sobre el eficientismo penal de enemigo. Grupo Editorial IBAÑEZ. Bogotá 2006, S. 15-20.

Eser, A., Burchard C.: Interlokales „ne bis in idem“ in Europa? Von „westfälischem“ Souveränitätspathos zu europäischem Gemeinschaftsdenken. In: Derra, H.-J. (Hrsg.), Freiheit, Sicherheit und Recht. Festschrift für Jürgen Meyer zum 70. Geburtstag. Nomos. Baden-Baden 2006, S. $499-524$

Eser, A. and G. Heine: Strafbarer Eigennutz ( $\$ \$$ 284-290), 2444-2463, Wilderei ( $\$ \$ 292-297), 2474-2483$, Gefährdung schutzbedürftiger Gebiete ( $\$ 329), 2210-2219$. In: Schönke, A., Schröder, H., Eser, A., et al., Strafgesetzbuch, Kommentar. 27. Aufl. Verlag C.H. Beck. München 2006. 
Eser, A., Schönke, A., Schröder, et al. (Hrsg.), Strafgesetzbuch, Kommentar. 27. Aufl. Verlag C.H. Beck, München 2006 (2882 S.)

Eser, A., Sieber, U., Arnold, J. (Hrsg.): Strafrecht in Reaktion auf Systemunrecht. Vergleichende Einblicke in Transitionsprozesse. Teilband 9: China (Richter). Schriftenreihe des Max-Planck-Instituts für ausländisches und internationales Strafrecht. Strafrechtliche Forschungsberichte S 82.9. Duncker \& Humblot. Berlin 2006 (267 S.).

Eser, A., Sieber, U., Arnold, J. (Hrsg.): Strafrecht in Reaktion auf Systemunrecht. Vergleichende Einblicke in Transitionsprozesse. Teilband 10: Südkorea (Cho/Holzapfl/ Richter). Schriftenreihe des Max-Planck-Instituts für ausländisches und internationales Strafrecht. Strafrechtliche Forschungsberichte S 82.10. Duncker \& Humblot. Berlin 2006 (126 S.).

Eser, A., Sieber, U., Kreicker, H. (Hrsg.): Nationale Strafverfolgung völkerrechtlicher Verbrechen. National Prosecution of International Crimes. Teilband 7: Völkerstrafrecht im Ländervergleich (Kreicker). Schriftenreihe des Max-Planck-Instituts für ausländisches und internationales Strafrecht. Strafrechtliche Forschungsberichte S 95.7. Duncker \& Humblot. Berlin 2006 (424 S.).

\section{7}

Eser, A.: Gedanken im Übergang. Gekürzte Fassung der Rede zum Amtswechsel am Freiburger Max-Planck-Institut für ausländisches und internationales Strafrecht, 2004. In: Freilaw, Freiburger Law Students Journal, 17-24 (2007).

Eser, A.: Vorzugswürdigkeit des adversatorischen Prozesssystems in der internationalen Strafjustiz? Reflektionen eines Richters. In: Müller-Dietz, H. et al. (Hrsg.), Festschrift für Heike Jung. Nomos. Baden-Baden 2007, S. 167-187.

Eser, A.: Schönke/Schröder, Strafgesetzbuch. In: Willoweit, D. (Hrsg.), Rechtswissenschaft und Rechtsliteratur im 20. Jahrhundert. Mit Beiträgen zur Entwicklung des Verlages C.H. Beck. Verlag C.H. Beck. München 2007, S. 851-865.

Eser, A.: The Nature and Rationale of Punishment. In: Cardozo Law Review 28, 2427-2436 (2007).

Eser, A.: Uluslararasi Cezea Mahkemesinin Kurulmasi: Roma Statüsünün Ortaya Csikişi Ve Temel Özellikleri. In: Yenisey, F. (Hrsg.), Uluslararası Ceza Divanı. Arıkan Verlag. Istanbul 2007, S. 3-69.

Eser, A.: Uluslararasi Ceza Adalet Sisteminde Iddia Makaminin Oynadigi Temel Rol. In: Yenisey, F. (Hrsg.), Uluslararası Ceza Divanı. Arıkan Verlag Istanbul 2007, S. 281-295.

Eser, A., Arnold, J.: Einführung in das Gesamtprojekt. In: Eser, A., Sieber, U., Arnold, J. (Hrsg.), Strafrecht in Reaktion auf Systemunrecht. Vergleichende Einblicke in Transitionsprozesse. Teilband 11: Chile (Millaleo Hernández), Uruguay (Fernández). Schriftenreihe des Max-PlanckInstituts für ausländisches und internationales Strafrecht. Strafrechtliche Forschungsberichte S 82.11. Duncker \& Humblot. Berlin 2007, S. 1-16.

Eser, A., Koch H.-G., Seith C. (Hrsg.): Internationale Perspektiven zu Status und Schutz des extrakorporalen Embryos - International Perspectives on the Status and Protection of the Extracorporeal Embryo. Rechtliche Regelungen und Stand der Debatte im Ausland. Ethik und Recht in der Medizin 42/1. Nomos. Baden-Baden 2007 (500 S.).

Eser, A., Sieber, U., Arnold, J. (Hrsg.): Strafrecht in Reaktion auf Systemunrecht. Vergleichende Einblicke in Transitionsprozesse. Teilband 11: Chile (Millaleo Hernández), Uruguay (Fernández). Schriftenreihe des Max-PlanckInstituts für ausländisches und internationales Strafrecht. Strafrechtliche Forschungsberichte S 82.11. Duncker \& Humblot. Berlin 2007 (643 S.).

\section{Fan, Wen}

\section{6}

Fan, W.: Todesstrafe in strafrechtsdogmatischer und kriminologischer Perspektive. In: Criminal Law Review 18 1-12 (2006)

Fan, W.: Verfall und Einziehung in Deutschland Grundlage, Strukturen und ihre Anwendung. In: Chen, Z (Hrsg.), New Reports in Criminal Law. Chinese People's Public Security University Press. Beijing 2006, S. 425-456.

\section{Galain Palermo, Pablo} 2006

Galain Palermo, P.: Informe Uruguay. In: Ambos, Malarino, Woischnick (Hrsg.), Dificultades jurídicas y políticas para la ratificación o implementación del estatuto de roma de la corte penal internacional. Contribuciones de América latina y Alemania. Konrad-Adenauer-Stiftung. Montevideo 2006, S. 411-447.

Galain Palermo, P.: Responsabilidad penal de las personas jurídicas. In: Revista Penal 17, 245-248 (2006).

Galain Palermo, P.: Reformas legales en Uruguay entre 2003 y 2006. In: Revista Penal 18, S. 294-299 (2006).

Galain Palermo, P.: Formas de consenso que permiten la suspensión del proceso penal en Alemania y Portugal. Algunos lineamientos que podrían ser considerados por el legislador nacional, considerando la necesidad de una urgente reforma del proceso penal uruguayo. In: Revista do Ministerio Público, 106, 43-81, (2006).

\section{7}

Galain Palermo, P.: La teoría del error en el sistema penal uruguayo. In: Revista Penal 20, 227-230 (2007).

Galain Palermo, P.: Suspensión del proceso y tercera vía: avances y retrocesos del sistema penal. In: Revista Penal 20, 58-73 (2007).

Galain Palermo, P.: Cooperación y asistencia judicial con la corte penal internacional: Uruguay. In: Ambos, K., Malarino, E., Elsner, G. (Hrsg.), Cooperación y asistencia judicial con la corte penal internacional. Contribuciones de América latina, Alemania, España e Italia. Fundación Konrad Adenauer. Montevideo 2007, S. 493-556.

Galain Palermo, P.: Insolvencias Punibles. In: Revista Penal 19, 222-225 (2007).

Galain Palermo, P.: Reparation as a functional equivalent of punishment: a proposal set between two models of justice. In: China University of Political Science and Law (Hrsg.), New theoretical system of criminal law in the age of globalization. Beijing 2007, S. 21-26.

Galain Palermo, P., Romero, A: Criminalidad organizada y reparación. Hacia una propuesta politico-criminal que disminuya la incompatibilidad entre ambos conceptos. In Pérez Álvarez, F., et al. (Hrsg.), Universitas Vitae. Homenaje a Ruperto Núñez Barbero. Ediciones Universidad de Salamanca. Salamanca 2007, S. 245-278.

\section{Gencheva, Irina}

\section{7}

Gencheva, I., Gruev, L.: Die bulgarische Strafprozessordnung, in Kraft getreten am 29. April 2006. Übersetzung von Irina Gencheva, Einführung von Lazar Gruev. Schriftenreihe des Max-Planck-Instituts für ausländisches und internationales Strafrecht. Sammlung ausländischer Strafgesetzbücher in deutscher Übersetzung G 117. Duncker \& Humblot. Berlin 2007 (196 S.). 


\section{Gröseling, Nadine} 2007

Gröseling, N., Höfinger, F. M.: Hacking und Computerspionage. Auswirkungen des 41. StrÄndG zur Bekämpfung der Computerkriminalität. In: Multimedia und Recht 10, 9 , 549-553 (2007).

Gröseling, N., Höfinger, F. M.: Computersabotage und Vorfeldkriminalisierung. Auswirkungen des 41. StrÄndG zur Bekämpfung der Computerkriminalität. In: Multimedia und Recht 10, 10, 626-630 (2007).

Gröseling, N., Höfinger, F.M.: Der strafbare Umgang mit „Hacker-Tools“ auf dem Prüfstand. In: Multimedia und Recht 10, 12, XXVII-XXX (2007).

\section{Grundies, Volker}

2007

Grundies, V., Albrecht, H.-J.: Sexuelle Gewaltkriminalität im Lebenslängsschnitt. Die Entwicklung von Sexualkriminalität an Hand von Daten der Freiburger Kohorten studie. In: Lösel, F., Bender, D., Jehle, J.-M. (Hrsg.) Kriminologie und wissensbasierte Kriminalpolitik. Entwicklungs- und Evaluationsforschung. Neue Kriminologische Schriftenreihe. Forum Verlag Godesberg. Mönchengladbach 2007, S. 447-475.

Grundies, V., Titterington, V. B.: An Exploratory Analysis of German and U.S. Youthful Homicide Offending. In Homicide Studies 11, 189-212 (2007).

\section{Herz, Annette Louise} 2006

Herz, A. L.: Menschenhandel. Eine empirische Untersuchung zur Strafverfolgungspraxis. forschung aktuell | research in brief 32. edition iuscrim. Freiburg i. Br. 2006 (37 S.).

Herz, A. L.: Trafficking in Human Beings. An Empirical Study on Criminal Prosecution in Germany. forschung aktuell $\mid$ research in brief 33. edition iuscrim. Freiburg i. Br 2006 (28 S.).

\section{Höfinger, Frank Michael}

2007

Höfinger, F. M., Gröseling, N.: Hacking und Computerspionage. Auswirkungen des 41. StrÄndG zur Bekämpfung der Computerkriminalität. In: Multimedia und Recht $10,9,549-553$ (2007)

Höfinger, F. M., Gröseling, N.: Computersabotage und Vorfeldkriminalisierung. Auswirkungen des 41. StrÄndG zur Bekämpfung der Computerkriminalität. In: Multimedia und Recht 10, 10, 626-630 (2007).

Höfinger, F.M., Gröseling, N.: Der strafbare Umgang mit „Hacker-Tools“ auf dem Prüfstand. In: Multimedia und Recht 10, 12, XXVII-XXX (2007).

Höfinger, F. M., Sieber, U.: Allgemeine Grundsätze der Haftung, Kapitel 18.1. In: Hoeren, T., Sieber, U. (Hrsg.) Handbuch Multimedia-Recht, 18. Aufl. 2007. Verlag C.H Beck. München 2007, S. 1-72.

\section{Holzapfl, Carolin}

2006

Holzapfl, C., Cho, B., Richter, T.: Südkorea. In: Eser, A., Sieber, U., Arnold, J. (Hrsg.), Strafrecht in Reaktion auf Systemunrecht. Vergleichende Einblicke in Transitionspro- zesse. Teilband 10: Südkorea (Cho/Holzapfl/Richter). Schriftenreihe des Max-Planck-Instituts für ausländisches und internationales Strafrecht. Strafrechtliche Forschungsberichte S 82.10. Duncker \& Humblot. Berlin 2006, 126 S.

\section{Huber, Barbara} 2006

Huber, B.: England \& Wales. In: Gropp, W., Sinn, A (Hrsg.), Organisierte Kriminalität und kriminelle Organe. Präventive und repressive Maßnahmen vor dem Hintergrund des 11. September 2001. Nomos. Baden-Baden 2006, S. 149-172.

Huber, B.: The normative framework against child abuse in European supranational and German law - an overview. In: Digesta Turcica 2 (2006) 225-239.

Huber, B., Nakamura, H.: Die japanische ZPO in deutscher Sprache. Heymanns Verlag. Köln 2006 (266 S.).

\section{7}

Huber, B.: Die Vorbereitung von Wiederaufnahmeverfahren in England - ein Beispiel für ausgelagerte Justizaufgaben. In: Müller-Dietz, H. et al. (Hrsg.), Festschrift für Heike Jung. Nomos. Baden-Baden 2007, S. 331-344.

\section{Jähnke, Jochen} 2006

Jähnke, J., Gartmann, C. (Hrsg.): 22. DV-Treffen der Max-Planck-Institute. 16.-18. November 2005 in Göttingen. In: GWDG-Berichte 70. Gesellschaft für wissenschaftliche Datenverarbeitung mbH. Göttingen 2006 (140 S.).

Jähnke, J., Köllisch, T.: Cybercrime gegen Organisationen. Ergebnisse ausländischer Viktimisierungsuntersuchungen und Überlegungen für einen Survey in Deutschland. In: Monatsschrift für Kriminologie und Strafrechtsreform 89, 5, 366-388 (2006).

\section{Jarvers, Konstanze 2007}

Jarvers, K.: Massen- und Kleinkriminalität vor dem italienischen Friedensrichter. Neue Wege durch alternative Erledigungsmöglichkeiten und Sanktionsformen. Schriftenreihe des Max-Planck-Instituts für ausländisches und internationales Strafrecht. Strafrechtliche Forschungsberichte S 108. Duncker \& Humblot. Berlin 2007 (308 S.).

\section{Kafatou-Haeusermann, Maria} 2007

Kafatou-Haeusermann, M.: The Media-Crime Nexus Revisited: On the Re-Construction of Crime and Law-andOrder in Crime-Appeal Programming. Schriftenreihe des Max-Planck-Instituts für ausländisches und internationales Strafrecht. Kriminologische Forschungsberichte K 133. Duncker \& Humblot. Berlin 2007 (521 S.).

\section{Kaiser, Günther †} 2006

Kaiser, G.: „Strafrecht und Kriminologie unter einem Dach“ aus der Perspektive des Kriminologen. In: Sieber, U., Albrecht, H.-J. (Hrsg.), Strafrecht und Kriminologie unter einem Dach. Kolloquium zum 90. Geburtstag von Prof. Dr. Hans-Heinrich Jescheck. Schriftenreihe des Max-PlanckInstituts für ausländisches und internationales Strafrecht. 
Interdisziplinäre Forschungsberichte aus Strafrecht und Kriminologie I 14. Duncker \& Humblot. Berlin 2006, S. 66-77.

Kaiser, G.: Menschenrechte im europäischen Strafvollzug - Gewährleistung oder Gefährdung durch Staat, Gesellschaft und Staatengemeinschaft? In: Derra, H.-J. (Hrsg.), Freiheit, Sicherheit und Recht. Festschrift für Jürgen Meyer zum 70. Geburtstag. Nomos. Baden-Baden 2006, S. 133-157.

Kaiser, G.: Wo steht die Kriminologie, und wohin geht sie? In: Obergfell-Fuchs, J., Brandenstein, M. (Hrsg.), Nationale und internationale Entwicklungen in der Kriminologie - Festschrift für Helmut Kury zum 65. Geburtstag. Verlag für Polizeiwissenschaft. Frankfurt a.M. 2006, S. 19-34.

Kaiser, G.: Wirtschaftskriminalität im Blickpunkt. In: Saito, S., Hidaka, Y., Kai, K. (Hrsg.), Festschrift für Prof. Toshio Kamiyama. Seibundo. Tokio 2006, S. 393-409.

Kaiser, G.: Rezension zu: James Q. Whitman, Harsh Justice. Criminal Punishment and the Widening Divide Between America and Europe. Oxford, Oxford University Press, 2003. In: Monatsschrift für Kriminologie und Strafrechtsreform 89, 2, 152-155 (2006).

Kaiser, G.: Kontinuität und Diskontinuität in den Diskursen über Kriminalität und strafrechtliche Sozialkontrolle im Lichte wissenschaftshistorischer Betrachtung - zugleich Besprechung des Buches von Imanuel Baumann. Dem Verbrechen auf der Spur. Eine Geschichte der Kriminologie und Kriminalpolitik in Deutschland 1880-1980, Göttingen 2006. In: Monatsschrift für Kriminologie und Strafrechtsreform 89, 4, 314-327 (2006).

Kaiser, G.: Rezension zu: Peter Becker, Richard F. Wetzell, Criminals and their Scientists. The History of Criminology in International Perspective, Cambridge, Cambridge University Press, 2006. In: Monatsschrift für Kriminologie und Strafrechtsreform 89, 5, 406-412 (2006).

Kaiser, G.: Rezension zu: Thomas Feltes, Christian Pfeiffer, Gernot Steinhilper (Hrsg.), Kriminalpolitik und ihre wissenschaftlichen Grundlagen. Festschrift für HansDieter Schwind zum 70. Geburtstag, Heidelberg 2006, XVI, 1204 S. In: Monatsschrift für Kriminologie und Strafrechtsreform 89, 6, 477-478 (2006).

Kaiser, G.: 40 Jahre gemeinsame Kolloquien Südwestdeutscher und Schweizer kriminologischer Institutionen - Randnotizen zur Funktion kriminologischer Workshops, deren Anfänge, Inhalte und Veränderungen. In: Höfer, S., Spieß, G. (Hrsg.), Neuere Kriminologische Forschung im Südwesten. Eine Darstellung der Forschungsarbeit aus Anlass des 40. Kolloquiums der Südwestdeutschen und benachbarten Kriminologischen Institute. edition iuscrim. Freiburg i. Br. 2006, S. 1-16.

Kaiser, G., Schöch, H.: Juristischer Studienkurs „Kriminologie, Jugendstrafrecht, Strafvollzug“. 6. Aufl. Verlag C.H. Beck. München 2006 (278 S.).

\section{7}

Kaiser, G.: Kriminologie: Begriff und Aufgaben. In: Schneider, H. J. (Hrsg.), Internationales Handbuch der Kriminologie. De Gruyter Recht. Berlin 2007, S. 25-52.

Kaiser, G.: Strafrechtliche Sozialkontrolle unter den Bedingungen der Globalisierung. In: Müller-Dietz, H. et al. (Hrsg.), Festschrift für Heike Jung. Nomos. Baden-Baden 2007, S. 379-395.

Kaiser, G.: Einführung. In: Deutscher Taschenbuch Verlag (Hrsg.), Strafvollzugsgesetz mit Verwaltungsvorschriften. 18. Aufl. Deutscher Taschenbuch Verlag. München 2007, S. IX-XXVII.

Kaiser, G.: Erziehung und Strafe in der Postmoderne. In: Schöch, H., Helgerth, R., Dölling, D., König, P. (Hrsg.), Festschrift für Reinhard Böttcher zum 70. Geburtstag am 29. Juli 2007. De Gruyter Recht. Berlin 2007, S. 283-301.
Kaiser, G.: Postmoderne Kriminologie oder Kriminologie in der Postmoderne? In: Krajewski, K. (Hrsg.), Nauki penalne wobec problemów współczesnej przestępczości. Księga jubileuszowa z okazij 70. rocznicy urodzin profesora Andrzeja Gaberle. Festschrift für Professor Andrzej Gaberle zum 70. Geburtstag. Wolters Kluwer Polska Warschau 2007, S. 429-446.

Kaiser, G.: Moderne und postmoderne Kriminalpolitik als Probleme des Strukturvergleichs. In: Criminology: Yesterday, Today, Tomorrow 12, 11-32 (2007).

Kaiser, G.: Rezension zu: Christian Bachhiesl, Zur Konstruktion der kriminellen Persönlichkeit, Die Kriminalbiologie an der Karl-Franzens-Universität Graz, Hamburg, Verlag Dr. Kovac, 2005, 354 S., David von Mayenburg, Kriminologie und Strafrecht zwischen Kaiserreich und Nationalsozialismus, Hans von Hentig (1887-1974), Baden-Baden, Nomos Verlagsgesellschaft, 2006, 492 S., Kai Naumann, Gefängnis und Gesellschaft. Freiheitsentzug in Deutschland in Wissenschaft und Praxis 1920-1960 Berlin, LIT Verlag, 2006, 335 S. In: Monatsschrift für Kriminologie und Strafrechtsreform 90, 62-73 (2007).

Kaiser, G.: 40 Jahre gemeinsame Kolloquien Südwestdeutscher und Schweizer kriminologischer Institutionen - Randnotizen zur Funktion kriminologischer Workshops, deren Anfänge, Inhalte und Veränderungen. In: Höfer, S., Spieß, G. (Hrsg.), Neuere Kriminologische Forschung im Südwesten. Eine Darstellung der Forschungsarbeit aus Anlass des 40. Kolloquiums der Südwestdeutschen und benachbarten Kriminologischen Institute. 2. akt. Aufl. edition iuscrim. Freiburg i. Br. 2007, S. 1-16.

\section{Kilchling, Michael}

\section{6}

Kilchling, M.: Die Rechtslage zur Opferbegleitung in Deutschland. In: Jesionek, U., Hilf, M. (Hrsg.), Die Begleitung des Verbrechensopfers durch den Strafprozess. Schriftenreihe der Weisser Ring Forschungsgesellschaft - VOR 2. Studienverlag. Innsbruck et al. 2006, S. 139-164.

Kilchling, M.: Rechtliche Instrumente zur Bekämpfung der Terrorismusfinanzierung im internationalen Vergleich. In: Gehl, G. (Hrsg.), Terrorismus - Krieg des 21. Jahrhunderts? Bertuch-Verlag. Weimar 2006, S. 87-113.

Kilchling, M., Albrecht, H.-J.: Victims of Terrorism - Policies and Legislation in Europe: Ann Overview on Victim-Related Assistance and Support (Expert Report). In: Council of Europe (Hrsg.), Victims - Support and assistance. Council of Europe Publishing. Strasbourg 2006, S. 199-250.

Kilchling, M., Albrecht, H.-J.: Institutions and organisations responsible for victims (Contacts). In: Council of Europe (Hrsg.), Victims - Support and assistance. Council of Europe Publishing. Strasbourg 2006, S. 253-262.

Kilchling, M., Albrecht, H.-J.: Victimes du terrorisme - Politiques et législations européennes: Tour d'horizon des systèmes d'assistance aux victimes (Rapport d'expertise). In: Conseil de l'Europe (Hrsg.), Soutien et aide aux victimes. Les Éditions du Conseil de l'Europe. Strasbourg 2006, S. 209-266.

Kilchling, M., Albrecht, H.-J.: Institutions et organisations responsables pour les victimes [Contacts]. In: Conseil de l'Europe (Hrsg.), Soutien et aide aux victimes. Les Éditions du Conseil de l'Europe. Strasbourg 2006, S. 269-278.

\section{7}

Kilchling, M., Albrecht, H.-J.: Victims of Terrorism Policies - Should Victims of Terrorism be Treated Differently? In: European Journal on Criminal Policy and Research 13, 1-2, 13-31 (2007). 


\section{Kinzig, Jörg}

2006

Kinzig, J.: Stalking - ein Fall für das Strafrecht? In Zeitschrift für Rechtspolitik 39, 255-258 (2006)

Kinzig, J.: „Die Sicherungsverwahrung - von einer vergessenen zu einer boomenden Maßregel“. In: Barton, S. (Hrsg.), ,... weil er für die Allgemeinheit gefährlich ist!“ Nomos. Baden-Baden 2006, S. 143-173.

Kinzig, J.: „Kurzer Prozess in Verkehrsstrafsachen?“ ein internationaler Vergleich. In: Deutsche Akademie für Verkehrswissenschaft (Hrsg.), 44. Deutscher Verkehrsgerichtstag 2006. Deutsche Akademie für Verkehrswissenschaft. Hamburg 2006, S. 252-265.

Kinzig, J.: Organisierte Kriminalität in Deutschland Ergebnisse einer normativ-empirischen Untersuchung. In Gehl, G. (Hrsg.), Europa im Griff der organisierten Kriminalität? Bertuch Verlag GmbH. Weimar 2006, S. 19-37.

\section{7}

Kinzig, J.: Anmerkung zu BGH B vom 19.07.2006 - 1 StR 238/06. In: Strafverteidiger 27, 575-577 (2007).

Kinzig, J.: Entwicklung, Stand und Perspektiven einer Sicherungsverwahrung für Jugendliche und Heranwachsende. In: Recht der Jugend und des Bildungswesens 55, 2 155-166 (2007)

Kinzig, J.: Anmerkung zu BGH U v. 21.12.2006 - 3 StR 396/06. In: Juristenzeitung 62, 1006-1008 (2007).

Kinzig, J.: Lexikonartikel „Freiheitsstrafe“, 348-349, Lexikonartikel „Geldstrafe“, 375, Lexikonartikel „Maßregeln der Besserung und Sicherung“, 628-629, Lexikonartike „Schuldunfähigkeit“, 792-793, Lexikonartikel „Sicherungsverwahrung“, 824-825. In: Deutscher Verein für öffentliche und private Fürsorge e. V. (Hrsg.), Fachlexikon der sozialen Arbeit. Nomos. Baden-Baden 2007.

Kinzig, J.: Anmerkung zum Beschl. des BVerfG v. 8.11.2006 - 2 BvR 578/02 - 2 BvR 796/02. In: Juristische Rundschau, 165-169 (2007).

Kinzig, J.: Brauchen wir einen Straftatbestand gegen Stalking? In: Richter ohne Robe. Zeitschrift des Bundesverbandes Ehrenamtlicher Richterinnen und Richter e.V. - Deutsche Vereinigung der Schöffinnen und Schöffen (DVS) 19, 1, 3-9 (2007)

Kinzig, J.: Analyse de quelques dispositifs visant à lutter contre la criminalité organisée en Allemagne. In: Cesoni, M. L. (Hrsg.), Nouvelles méthodes de lutte contre la criminalité: La normalisation de l'exception. Bruylant. Bruxelles 2007, S. 303-345.

Kinzig, J., Grupp, M.: Der Griff ins Lenkrad - zugleich Anmerkung zu den Entscheidungen BGH, Beschluss vom 13.06.2006 (4 StR 123/06) und OLG Dresden, NJW 2006, 1013. In: Neue Zeitschrift für Strafrecht 27, 132-136 (2007).

Kinzig, J., Höfer, S.: Wer ist denn der Richter? Strafzumessung im Betäubungsmittelstrafrecht. In: Vereinigung Baden-Württembergischer Strafverteidiger e.V. und die Mitglieder des Strafrechtsausschusses des Deutschen An waltvereins (Hrsg.), 1. Symposium Betäubungsmittelstrafrecht. DesignConcepts. St. Märgen 2007, S. 19-48.

Kinzig, J., Weber, J.: Strafrechtlicher Fall zu den Themen Strafantrag und Sanktionen. In: ius.full - Forum für Juristische Bildung 5, 1, 8-20 (2007).

Kinzig, J., Zander, S.: Der neue Tatbestand der Nachstellung ( $\$ 238$ StGB). In: Juristische Arbeitsblätter, 481-488 (2007).

\section{Kiriakaki, Irini \\ 2006}

Kiriakaki, I.: Das Humanexperiment als völkerstrafrechtliches Verbrechen - Vom Nürnberger Kodex zum Rom-Statut für einen Internationalen Strafoerichtshof. Ein Kommentar zu den Tatbeständen von Art. 8(2)(b)(x) bzw. (e)(xii) IStGHStatut aus medizinrechtlicher Sicht. In: Zeitschrift für die Gesamte Strafrechtswissenschaft 118, 1, 229-261 (2006).

\section{7}

Kiriakaki, I.: Der Schutz des Menschen und des Embryos invitro in der medizinischen Forschung. Eine rechtsvergleichende Untersuchung des Menschenrechtsübereinkommens zur Biomedizin, des griechischen und des deutschen Rechts. In: Ethik und Recht in der Medizin 43. Nomos. Baden-Baden 2007 (757 S.)

\section{Knust, Nandor} 2007

Knust, N., Rabbat, P.J.: Beyond the Charter: Peace, Security and the Role of Justice: Bericht über die dritte Jahreskonferenz der Hague Academic Coalition. In: Zeitschrift für die Gesamte Strafrechtswissenschaft 119, 1, 186-193 (2007).

\section{Koch, Hans-Georg} 2006

Koch, H.-G.: Lebensbeginn und Lebensende als Probleme des Medizin(straf)rechts. In: Frisch, Wolfgang. (Hrsg.), Gegenwartsfragen des Medizinstrafrechts. Portugiesisch-deutsches Symposium zu Ehren von Albin Eser in Coimbra. Ethik und Recht in der Medizin 41. Nomos. Baden-Baden 2006, S. 127-141.

Koch, H.-G.: Aufgaben und Verpflichtung der EthikKommission - aus der Sicht des Rechts. In: Just, H., Kindt, H., Koch, H.-G. (Hrsg.), Selbstkontrolle der Wissenschaft in der medizinischen Forschung. Erfahrungen und Perspektiven aus 25 Jahren Ethik-Kommission. Schriftenreihe der EthikKommission der Albert-Ludwigs-Universität 3. Eigenverlag (Albert-Ludwigs-Universität). Freiburg i. Br. 2006, S. 43-54.

Koch, H.-G.: Die rechtlichen Aspekte der Transplantation in Deutschland. In: Krukemeyer, M., Lison, A.E. (Hrsg.), Transplantationsmedizin - Ein Leitfaden für den Praktiker. De Gruyter. Berlin et al. 2006, S. 25-40.

Koch, H.-G., Just, H., Kindt, H. (Hrsg.): Selbstkontrolle der Wissenschaft in der medizinischen Forschung. Erfahrungen und Perspektiven aus 25 Jahren Ethik-Kommission. Schriftenreihe der Ethik-Kommission der Albert-Ludwigs Universität 3. Eigenverlag (Albert-Ludwigs-Universität) Freiburg i. Br. 2006 (128 S.)

Koch, H.-G., Sieber, U., Simon, J.-M.: Strafbare Mitwirkung von Führungspersonen in Straftätergruppen und Netzwerken: Eine rechtsvergleichende Analyse. In: MaxPlanck-Gesellschaft zur Förderung der Wissenschaften e.V. (Hrsg.), Jahrbuch 2006 der Max-Planck-Gesellschaft - CDROM-Anlage. Max-Planck-Gesellschaft zur Förderung der Wissenschaften e.V. München 2006 (4 S.).

\section{7}

Koch, H.-G.: Disziplinspezifische Vorannahmen: Intrinsische und extrinsische Statusbestimmung des extrakorporalen Embryos - Vermittlung und Kombinatorik der verschiedenen Kriterien. In: Maio, G. (Hrsg.), Der Status des extrakorporalen Embryos - Perspektiven eines interdisziplinären Zugangs. frommann-holzboog. Stuttgart-Bad Cannstatt 2007, S. 47-64. 
Koch, H.-G.: Einfachgesetzliche Regelungsoptionen für den Gesetzgeber. In: Maio, G. (Hrsg.), Der Status des extrakorporalen Embryos - Perspektiven eines interdisziplinären Zugangs. frommann-holzboog. Stuttgart-Bad Cannstatt 2007, S. 657-671

Koch, H.-G.: Das deutsche Embryonenschutzgesetz im Rechtsvergleich. In: Nova Acta Leopoldina 96, 354 (2007) 229-235.

Koch, H.-G.: Rechtliche Grundlagen der Arzthaftung für Behandlungsfehler. In: Zeitschrift für Medizinische Ethik 53, 207-217 (2007).

Koch, H.-G.: A Comparative Legal Analysis of AntiDoping Activities - General Framework and Criminal Law Aspects. In: Vieweg, K., Siekmann, R. (Hrsg.), Legal Comparison and the Harmonisation of Doping Rules. Pilot Study for the European Commission. Duncker \& Humblot. Berlin 2007, S. 95-158.

Koch, H.-G., Eser, A., Seith, C. (Hrsg.): Internationale Perspektiven zu Status und Schutz des extrakorporalen Embryos - International Perspectives on the Status and Protection of the Extracorporeal Embryo. Rechtliche Regelungen und Stand der Debatte im Ausland. Ethik und Recht in der Medizin 42/1. Nomos. Baden-Baden 2007 (500 S.).

\section{Köllisch, Tilmann} 2006

Köllisch, T., Jähnke, J.: Cybercrime gegen Organisationen. Ergebnisse ausländischer Viktimisierungsuntersuchungen und Überlegungen für einen Survey in Deutschland. In: Monatsschrift für Kriminologie und Strafrechtsreform 89, 5, 366-388 (2006).

\section{7}

Köllisch, T.: Risikomanagement und punitive Segregation als „Kriminalpolitik von unten“: Zum Zeitverlauf des Hell-Dunkelfeldverhältnisses der Jugendgewalt in Deutschland zwischen 1986 und 2003. In: Kriminologisches Journal 39, 4, 243-259 (2007)

Köllisch, T.: Die Messbarkeit des Erfolgs kriminalpolitischer Interventionen am Beispiel der bundesdeutschen Heroinstudie. In: Lösel, F., Bender, D., Jehle, J.-M. (Hrsg.), Kriminologie und wissensbasierte Kriminalpolitik. Entwicklungs- und Evaluationsforschung. Neue Kriminologische Schriftenreihe. Forum Verlag Godesberg. Mönchengladbach 2007, S. 133-145.

Köllisch, T., Löbmann, R. Kreuzer, A.: Das bundesdeutsche Modellprojekt zur heroingestützten Behandlung Opiatabhängiger. Nomos. Baden-Baden 2007 (276 S.).

\section{Kouassi, Adome Blaise} 2006

Kouassi, A. B.: Kinder- und Jugendkriminalität - Die Strafbarkeit Minderjähriger und die Problematik der DrittZurechnung. Ein strafrechtswissenschaftlicher Vergleich zwischen Côte d'Ivoire (Elfenbeinküste), Deutschland, Frankreich und Italien. In: Kriminologija (Criminology: Yesterday, Today, Tomorrow, St. Petersburg) 11, 2, 91-110 (2006).

\section{Kreicker, Helmut}

2006

Kreicker, H., Eser, A., Sieber, U. (Hrsg.): Nationale Strafverfolgung völkerrechtlicher Verbrechen. National Prosecution of International Crimes. Teilband 7: Völker- strafrecht im Ländervergleich (Kreicker). Schriftenreihe des Max-Planck-Instituts für ausländisches und internationales Strafrecht. Strafrechtliche Forschungsberichte S 95.7. Duncker \& Humblot. Berlin 2006 (424 S.).

\section{7}

Kreicker, H.: Völkerrechtliche Exemtionen. Grundlagen und Grenzen völkerrechtlicher Immunitäten und ihre Wirkungen im Strafrecht. Schriftenreihe des Max-PlanckInstituts für ausländisches und internationales Strafrecht. Strafrechtliche Forschungsberichte S 107. Duncker \& Humblot. Berlin 2007 (1538 S.).

\section{Kury, Helmut \\ 2006}

Kury, H.: (Jugend-)Gewalt in unserer Gesellschaft. Prävalenz, Entwicklung und Prävention. In: Ministerium für Inneres, Familie, Frauen und Sport, Geschäftsstelle „Beiräte zur Kriminalitätsverhütung“ (Hrsg.), Sicher leben im Saarland. 5. Fachtagung Kommunale Kriminalprävention: Steigende Gewalt(bereitschaft). 1. Dezember 2005. Eigenverlag des Ministeriums für Inneres, Familie, Frauen und Sport des Saarlandes. Saarbrücken 2006, S. 23-48.

Kury, H.: Gewalt in der Familie - Ergebnisse aus Deutschland. In: Karimov, E., Kury, H. (Hrsg.), Kriminalität und Kriminalprävention in Ländern des Umbruchs. Beiträge einer Internationalen Konferenz in Baku/Aserbaidschan. Brockmeyer Verlag. Bochum 2006, S. 127-164.

Kury, H., Brandenstein, M.: Ausmass, Entwicklung, Schadensintensität und strafrechtliche Behandlung der Verkehrskriminalität. In: Schweizerische Zeitschrift für Kriminologie 5, 1, 25-40 (2006).

Kury, H., Brandenstein, M.: Sobre la cuestión de una „Nueva Punitividad“ - Actitudes sancionadoras y política sancionadora. In: Guzmán Dálbora, J. L., Serrano Maíllo, A. (Hrsg.), Derecho penal y criminología como fundamento de la política criminal - Estudios en homenaje al Profesor Alfonso Serrano Gómez. Dykinson. Madrid 2006, S. 369-402.

Kury, H., Brandenstein, M.: Wahrnehmung und (Rechts-)Wirklichkeit der Verkehrsdelinquenz. In: Zeitschrift für Verkehrssicherheit 52, 1, 7-12 (2006)

Kury, H., Brandenstein, M., Yoshida, T.: Internationales Ausmaß und Bedeutung der Verkehrsdelinquenz (2). In: The Hokkai-Gakuen Law Journal XLII (117), 3, 773-790 (2006).

Kury, H., Brandenstein, M., Yoshida, T.: Internationales Ausmaß und Bedeutung der Verkehrsdelinquenz (1). In The Hokkai-Gakuen Law Journal XLII, 2, 214-240 (2006).

Kury, H. and U. Goritzka: Über die Aussagekraft von Bevölkerungsbefragungen - eine vergleichende Untersuchung zwischen Bremen und Rottweil. In: Hoffmann, R. (Hrsg.), Evaluation und Polizei. Konzeptionelle, methodische und empirische Einblicke in ein Forschungsfeld. Verlag für Polizeiwissenschaft. Frankfurt a.M. 2006, S. 79-100.

Kury, H., Karimov, E. (Hrsg.): Kriminalität und Kriminalprävention in Ländern des Umbruchs. Beiträge einer Internationalen Konferenz in Baku/Aserbaidschan. Brockmeyer Verlag. Bochum 2006 (495 S.).

Kury, H., Keller, M., Mitter, U., Rahmati, N.: Verbrechensfurcht, Punitivität und Einstellung zur Polizei - Ergebnisse einer Umfrage in Aserbaidschan. In: Karimov, E., Kury, H. (Hrsg.), Kriminalität und Kriminalprävention in Ländern des Umbruchs. Beiträge einer Internationalen Konferenz in Baku/Aserbaidschan. Brockmeyer Verlag. Bochum 2006, S. 415-486.

Kury, H., Obergfell-Fuchs, J.: Punitivität in Deutschland. Zur Diskussion um eine neue „Straflust“. In: Feltes, T., Pfeiffer, C. Steinhilper, G. (Hrsg.), Kriminalpolitik und 
ihre wissenschaftlichen Grundlagen. Festschrift für Prof Dr. Hans-Dieter Schwind zum 70. Geburtstag. C. F. Müller Verlag. Heidelberg 2006, S. 1021-1043.

\section{7}

Kury, H., Brandenstein, M., Yoshida, T.: Internationales Ausmaß und Bedeutung der Verkehrsdelinquenz (3). In: The Hokkai-Gakuen Law Journal XLII (118), 4, 115-138 (2007).

\section{Lukas, Tim}

2006

Lukas, T.: „Crime Prevention Carousel“ - Kriminalprävention in Großwohnsiedlungen. In: Forum Kriminalprävention, 1, 17-21 (2006)

\section{7}

Lukas, T. (Hrsg.): Crime Prevention in High-Rise Housing. Lessons from the Crime Prevention Carousel. Schriftenreihe des Max-Planck-Instituts für ausländisches und internationales Strafrecht. Kriminologische For schungsberichte K 136. Duncker \& Humblot. Berlin 2007 (132 S.).

\section{Macke, Julia}

2006

Macke, J.: European Union Agency for Fundamental Rights, 53-55, Exchange of Information on Criminal Records 76-78, Council of Europe, 81-86. In: eucrim - the European Criminal Law Associations' Forum, 03-04 (2006).

\section{7}

Macke, J.: European Union Agency for Fundamenta Rights. In: eucrim - the European Criminal Law Associations' Forum, 01-02, 18-19 (2007).

Macke, J.: Council of Europe. In: eucrim - the European Criminal Law Associations' Forum, 01-02, 41-47 (2007).

Macke, J.,Meyer, F.: Rechtliche Auswirkungen der Terroristenlisten im deutschen Recht. In: Höchstrichterliche Rechtsprechung im Strafrecht 8, 12, 445-465 (2007).

\section{Maljević, Almir}

2006

Maljević, A.: Fighting Money Laundering in Bosnia and Herzegovina: Controlling Criminals' Sloppiness or the Private Sector's Diligence? In: van Duyne, P.C., Maljevic, A. van Dijck, M., von Lampe, K., Newell, J. L. (Hrsg.), The Organisation of Crime for Profit. Conduct, Law and Measurement. Wolf Legal Publishers. Nijmegen 2006, S. 111-132.

Maljević, A.: Legal and Actual Treatment of Juveniles within the Juvenile Justice System of Bosnia and Herzegovina. In: Junger-Tas, J., Decker, S. H. (Hrsg.), Internationa Handbook of Juvenile Justice. Springer. Dordrecht 2006 , S. 415-438.

Maljević, A., Datzer, D., Muratbegović, E., Budimlić, M. Overtly about Police and Corruption. Association of Criminalists in Bosnia and Herzegovina. Sarajevo 2006 (265 S.).

Maljević, A., Datzer, D., Muratbegović, E., Budimlić, M. Otvoreno o policiji i korupciji. Association of Criminalists in Bosnia and Herzegovina. Sarajevo 2006 (221 S.).

Maljević, A., van Duyne, P. C., van Dijck, M., von Lampe, K., Newell, J. L. (Hrsg.): The Organisation of Crime for Profit. Conduct, Law and Measurement. Wolf Legal Publishers. Nijmegen 2006 (205 S.).

\section{7}

Maljević, A., Datzer, D., Muratbegovic, E. and Budimlic, M.: Harmonisation of the Criminal Legislation of Bosnia and Herzegovina with the European Anti-corruption Standards: Special Investigative Techniques and their Applicability on Corruption Cases. In: Tomášek, M. (Hrsg.), European Law and National Criminal Legislation. Univerzita Karlova, Právnická fakulta. Edi ní st edisko. Praha 2007, S. 209-224.

Maljević, A., Datzer, D., Muratbegovic, E. and Budimlic, M.: Factors Influencing Police Officers' Perception of Corruption: A Bosnian Perspective. In: Varstvoslovje 8, 3/4, 293-302 (2007).

Maljević, A., van Duyne, P.C., van Dijck, M., von Lampe, K., Harvey, J. (Hrsg.): Crime Business and Crime Money in Europe. The Dirty Linen of Illicit Enterprise. Wolf Legal Publishers. Nijmegen 2007 (267 S.).

\section{Manso Porto, Teresa} 2007

Manso Porto, T., Bacigalupo, E.: Die Rolle der Ehre im Strafrecht in Spanien. In: Tellenbach, S. (Hrsg.), Die Rolle der Ehre im Strafrecht. Schriftenreihe des Max-PlanckInstituts für ausländisches und internationales Strafrecht. Strafrechtliche Forschungsberichte S 111. Duncker \& Humblot. Berlin 2007, S. 589-618.

\section{Meyer, Frank}

2006

Meyer, F.: Plea Bargaining im Krieg gegen den Terror. In: Höchstrichterliche Rechtsprechung im Strafrecht, 5, 178-185 (2006).

Meyer, F.: Complementing Complementarity. In: International Criminal Law Review 6, 549-583 (2006).

Meyer, F.: „Gesprengte Fesseln?“ - Zur Zukunft des Guidelines Sentencing im U.S.-amerikanischen Strafzumessungsrecht. In: Zeitschrift für die Gesamte Strafrechtswissenschaft 118, 512-546 (2006).

Meyer, F.: Rezension zu: Katalin Ligeti, Strafrecht und strafrechtliche Zusammenarbeit in der Europäischen Union, Berlin, Duncker \& Humblot, Strafrechtliche Abhandlungen N.F. 164, 2005, 409 S. In: Höchstrichterliche Rechtsprechung im Strafrecht, 8/9, 321-324 (2006).

Meyer, F.: Freezing of Assets. In: eucrim - the European Criminal Law Associations' Forum, 03-04, 66-68 (2006).

\section{7}

Meyer, F.: Aussagefreiheit und gegenseitige Anerkennung. In: Goltdammer's Archiv 154, 15-35 (2007).

Meyer, F.: „Lost in Complexity“ - Gedanken zum Rechtsschutz gegen Smart Sanctions in der EU. In: Zeitschrift für Europarechtliche Studien 10, 1-69 (2007).

Meyer, F: Die Verantwortlichkeiten von Vertragsstaaten nach der Völkermordkonvention. Besprechung des Urteils des Internationalen Gerichtshofs in der Sache „Application of the Convention on the Prevention and Punishment of the Crime of Genocide (Bosnia and Herzegovina v. Serbia and Montenegro)“. In: Höchstrichterliche Rechtsprechung im Strafrecht 8, 5, 218-230 (2007).

Meyer, F.: Zurück zur gesetzlichen Beweistheorie? Beobachtungen zu den Legitimationsanforderungen an ein künftiges Abspracheverfahren. In: Zeitschrift für die Gesamte Strafrechtswissenschaft 119, 3, 633-663 (2007).

Meyer, F., Macke, J.: Rechtliche Auswirkungen der Terroristenlisten im deutschen Recht. In: Höchstrichterliche Rechtsprechung im Strafrecht 8, 12, 445-465 (2007). 


\section{Nolde, Malaika}

2007

Nolde, M.: Heimlicher Zugriff auf Computerdaten bei strafrechtlichen Ermittlungen (Kurzbeitrag zur Sondersitzung des Fachausschusses Strafrecht der Deutschen Gesellschaft für Recht und Informatik e.V.). In: Computer und Recht 23 (2007) R135-R136.

Nolde, M., Schnabel, C.: Verfassungsrechtliche Grenzen der staatlichen Inhaltskontrolle im Internet. In: Taeger, J., Wiebe, A. (Hrsg.), Aktuelle Entwicklungen im Informationstechnologierecht. Tagungsband Herbstakademie 2007. OlWIR Verlag für Wirtschaft, Informatik und Recht. Edewecht 2007, S. 285-300.

\section{Obergfell-Fuchs, Joachim} 2006

Obergfell-Fuchs, J., Brandenstein, M. (Hrsg.): Nationale und internationale Entwicklungen in der Kriminologie - Festschrift für Helmut Kury zum 65. Geburtstag. Verlag für Polizeiwissenschaft. Frankfurt a.M. 2006 (687 S.).

Obergfell-Fuchs, J., Brandenstein, M.: Helmut Kury zum 65. Geburtstag. In: Obergfell-Fuchs, J., Brandenstein M. (Hrsg.), Nationale und internationale Entwicklungen in der Kriminologie - Festschrift für Helmut Kury zum 65. Geburtstag. Verlag für Polizeiwissenschaft. Frankfurt a.M. 2006, S. 11-16.

\section{Oberwittler, Dietrich} 2007

Oberwittler, D.: The effects of neighbourhood poverty on adolescent problem behaviour - a multi-level analysis differentiated by gender and ethnicity. In: Housing Studies 22, 5, 781-803 (2007).

Oberwittler, D.: The Effects of Ethnic and Social Segregation on Children and Adolescents: Recent Research and Results from a German Multilevel Study (Discussion Paper Nr SP IV 2007-603). Wissenschaftszentrum Berlin für Sozialforschung. Berlin 2007 (46 S.)

Oberwittler, D.: Social Exclusion and Youth Crime in Europe. The Spatial Dimension. In: Aromaa, K. (Hrsg.), Penal policy, justice reform, and social exclusion. Plenary presentations held at the Fifth Annual Conference of the European Society of Criminology, 31 August - 3 September 2005, Krakow, Poland. European Institute for Crime Prevention and Control. Helsinki 2007, S. 27-44.

Oberwittler, D., Friedrichs, J.: Soziales Kapital in Wohngebieten. In: Franzen, A., Freitag, M. (Hrsg.), Sozialkapital. Special Issue 47 of Kölner Zeitschrift für Soziologie und Sozialpsychologie. VS Verlag für Sozialwissenschaften. Wiesbaden 2007, S. 450-486.

\section{Özsöz, Figen}

2007

Özsöz, F.: Rechtsextreme Gefangene im Strafvollzug. Ein Überblick. In: Monatsschrift für Kriminologie und Strafrechtsreform 90, 1, 30-47 (2007)

Özsöz, F.: Rechtsextremistische Gewalttäter im Jugendstrafvollzug - Verfestigung oder Ablösung rechtsextremistischer Orientierungen im Haftverlauf. In: Friedrich-EbertStiftung (Hrsg.), Der Aufstand der Zuständigen. Was kann der Rechtsstaat gegen Rechtsextremismus tun? Ergebnisse einer Konferenz der Friedrich-Ebert-Stiftung am 20. März 2007. Friedrich-Ebert-Stiftung. Berlin 2007, S. 95-103.
Özsöz, F., Brandenstein, M.: Hasskriminalität: Hasskriminalität: Auswirkungen von Hafterfahrungen auf fremdenfeindliche jugendliche Gewalttäter. In: Max-Planck-Gesellschaft zur Förderung der Wissenschaften e.V. (Hrsg.), Jahrbuch 2007 der Max-Planck-Gesellschaft - CD-ROMAnlage. Max-Planck-Gesellschaft zur Förderung der Wissenschaften e. V. München 2007 (5 S.).

\section{Paoli, Letizia}

2006

Paoli, L.: Organised Crime and its Control Policies in Europe. In: European Journal of Crime, Criminal Law and Criminal Justice 14, 3, 307-327 (2006).

Paoli, L.: Organised Crime Control Policies in Europe. In: Criminology in Europe 5, 1, 12-13 (2006).

Paoli, L.: Organised Crime and Anti-Crime Policies. In: Heywood, P. M., et al.(Hrsg.), Developments in European Politics. Palgrave. London 2006, S. 299-317.

Paoli, L.: Organisierte Kriminalität - Erscheinungsfomen und Ursachen. In: Heitmeyer, W., Schroettle, M. (Hrsg.), Gewalt zerstört: Berichte, Analysen, Prävention. Bundeszentrale für politische Bildung. Bonn 2006, S. 389-411.

Paoli, L., Schäfer, C.: Drogen und Strafverfolgung. Die Anwendung des \$3la BtMG und anderer Opportunitätsvorschriften auf Drogenkonsumentendelikte. forschung aktuell $\mid$ research in brief 34. edition iuscrim. Freiburg i. Br. 2006 (26 S.).

Paoli, L., Schäfer, C.: Drogenkonsum und Strafverfolgungspraxis. Eine Untersuchung zur Rechtswirklichkeit der Anwendung des \$31 a BTMG und anderer Opportunitätsvorschriften auf Drogenkonsumentendelikte. Schriftenreihe des Max-Planck-Instituts für ausländisches und internationales Strafrecht. Kriminologische Forschungsberichte K 130. Duncker \& Humblot. Berlin 2006 (460 S.).

\section{Pfützner, Peggy}

2006

Pfützner, P.: Der Europäische Haftbefehl und seine Umsetzung in das französische Recht. In: eucrim - the European Criminal Law Associations' Forum, 01-02, 29-33 (2006).

Pfützner, P.: Is the European Arrest Warrant void? In: Gruszczak, A. (Hrsg.), European Arrest Warrant - Achievements and Dilemmas. Working Paper 3 (7). European Centre-Natolin. Warsaw 2006, S. 18-30.

\section{Qi, Shenghui \\ 2006}

Qi, S.: Sentencing for Capital Cases in China. In: Chen, Z. (Hrsg.), Strengthening the Defence in Death Penalty Cases. Forum of Criminal Law Department of CASS - Chinese Academy of Social Sciences. Beijing 2006, S. 204-210.

\section{Rabbat, Paul J.}

\section{6}

Rabbat, P. J.: Le président de la Cour Pénale Internationale de passage à Montreal. In: Revue Agir, Amnistie Internationale $27,3,18-19$ (2006). 
2007

Rabbat, P. J., Knust, N.: Beyond the Charter: Peace Security and the Role of Justice: Bericht über die dritte Jahres konferenz der Hague Academic Coalition. In: Zeitschrift für die Gesamte Strafrechtswissenschaft 119, 1, 186-193 (2007).

\section{Rezaei, Hassan}

2006

Rezaei, H.: The Place of Women Rights in Thought of Abolhassan Banisadr. In: Iran Emrooz (Iranian Political Online Magazine), Seq. No.: 9727 (2006).

Rezaei, H.: Searching for Origins of Gender Violence in the Sharia Discourse. In: Albrecht, H.-J., Simon, J.-M., Rezaei, H, Rohne, H.-C., Kiza, E. (Hrsg.), Conflicts and Conflict Resolution in Middle Eastern Societies - Between Tradition and Modernity. Schriftenreihe des Max-PlanckInstituts für ausländisches und internationales Strafrecht Interdisziplinäre Forschungsberichte aus Strafrecht und Kriminologie I 13. Duncker \& Humblot. Berlin 2006 S. 317-335.

Rezaei, H.: Violent Conflict and Sulh in Middle Eastern Societies: A Critical Islamic Discourse Perspec tive. In: Albrecht, H.-J., Simon, J.-M., Rezaei, H, Rohne, H.-C., Kiza, E. (Hrsg.), Conflicts and Conflict Resolution in Middle Eastern Societies - Between Tradition and Modernity. Schriftenreihe des Max-Planck-Instituts für ausländisches und internationales Strafrecht. Interdisziplinäre Forschungsberichte aus Strafrecht und Kriminologie I 13 Duncker \& Humblot. Berlin 2006, S. 645-658.

Rezaei, H., Albrecht, H.-J., Simon, J.-M., Rohne, H.-C., Kiza, E. (Hrsg.): Conflicts and Conflict Resolution in Middle Eastern Societies - Between Tradition and Modernity. Schriftenreihe des Max-Planck-Instituts für ausländisches und internationales Strafrecht. Interdisziplinäre Forschungsberichte aus Strafrecht und Kriminologie I 13 Duncker \& Humblot. Berlin 2006 (660 S.).

\section{7}

Rezaei, H.: On the New Draft of Iran's Islamic Punishment Act. In: radiozamaneh.com, Seq. No.: 145 (2007).

Rezaei, H.: Blasphemy in traditional Sharia Criminal Law. In: radiozamaneh.com, Seq. No.: 181 (2007).

Rezaei, H.: Criminal Justice and Power (Part I and Part II). In: www.ayande.ir, Seq. No.: 181 and 189 (comments) (2007).

Rezaei, H.: Death Penalty in Islam and Iran. In: Enghelab Eslami 26, 687, 2-16 (2007).

Rezaei, H.: The Criminal Justice System in Japan (Part I). In: Criminal Sciences Review 1, 1, 1-34 (2007).

Rezaei, H.: Reason and Subjectivism in Islamic Criminal Theory. In: The Center for Cultural and Social Studies (Hrsg.), Individual and Reason in Islamic Thought The Center for Cultural and Social Studies. Tehran 2007 , S. 150-184.

\section{Richter, Thomas}

\section{6}

Richter, T.: China. Strafverfolgung von Systemunrecht der Kulturrevolution. In: Eser, A., Sieber, U., Arnold, J. (Hrsg.), Strafrecht in Reaktion auf Systemunrecht. Vergleichende Einblicke in Transitionsprozesse. Teilband 9: China (Richter). Schriftenreihe des Max-Planck-Instituts für ausländisches und internationales Strafrecht. Strafrechtliche Forschungsberichte S 82.9. Duncker \& Humblot. Berlin 2006, 267 S.
Richter, T.: Über Zustand und Tendenzen des chinesischen Strafrechts nach der Reform von 1997. In: Kansai University Review of Law and Politics 27, 69-80 (2006).

Richter, T., Holzapfl, C., Cho, B.: Südkorea. In: Eser, A., Sieber, U., Arnold, J. (Hrsg.), Strafrecht in Reaktion auf Systemunrecht. Vergleichende Einblicke in Transitionsprozesse. Teilband 10: Südkorea (Cho/Holzapfl/Richter). Schriftenreihe des Max-Planck-Instituts für ausländisches und internationales Strafrecht. Strafrechtliche Forschungsberichte S 82.10. Duncker \& Humblot. Berlin 2006, 126 S.

\section{Rohne, Holger-Christoph} 2006

Rohne, H.-C., Albrecht, H.-J., Simon, J.-M., Rezaei, H., Kiza, E. (Hrsg.): Conflicts and Conflict Resolution in Middle Eastern Societies - Between Tradition and Modernity. Schriftenreihe des Max-Planck-Instituts für ausländisches und internationales Strafrecht. Interdisziplinäre Forschungsberichte aus Strafrecht und Kriminologie I 13. Duncker \& Humblot. Berlin 2006 (660 S.).

\section{Sadr Touhid-Khaneh, Mohammad}

2007

Sadr Touhid-Khaneh, M.: Kurzlehrbuch zum Allgemeinen Teil des afghanischen Strafrechts (auf Dari). MaxPlanck-Institut für ausländisches öffentliches Recht und Völkerrecht. Heidelberg et al. 2007 (215 S.).

\section{Saux, María Soledad}

2006

Saux, M. S.: Estado de confusión en la legislación argentina. In: Cuarto Intermedio 41, 4, 36-37 (2006).

2007

Saux, M. S.: Immigration and Terrorism: A Constructed Connection. The Spanish Case. In: European Journal on Criminal Policy and Research 13, 1-2, 57-72 (2007).

\section{Schäfer-Vogel, Gundula} 2007

Schäfer-Vogel, G.: Gewalttätige Jugendkulturen - Symptom der Erosion kommunikativer Strukturen. Schriftenreihe des Max-Planck-Instituts für ausländisches und internationales Strafrecht. Kriminologische Forschungsberichte K 134. Duncker \& Humblot. Berlin 2007 (590 S.).

\section{Shea, Evelyne}

2007

Shea, E.: Why work? A Study of Prison Labour in England, France and Germany. Schriftenreihe des Max-PlanckInstituts für ausländisches und internationales Strafrecht. Kriminologische Forschungsberichte K 137. Duncker \& Humblot. Berlin 2007 (195 S.).

\section{Schulz, Stefan}

2006

Schulz, S.: Beyond Self-Control. Analysis and Critique of Gottfredson \& Hirschi's General Theory of Crime 
(1990). Schriftenreihe des Max-Planck-Instituts für ausländisches und internationales Strafrecht. Kriminologische Forschungsberichte K 131. Duncker \& Humblot. Berlin 2006 (306 S.).

\section{Seith, Carola}

2006

Seith, C.: Rezension zu: Peter Dabrock, Jens Ried (Hrsg.), Therapeutisches Klonen als Herausforderung für die Statusbestimmung des menschlichen Embryos, Paderborn, Mentis, 2005, 396 S. In: Zeitschrift für Medizinische Ethik 4, 417-421 (2006).

\section{7}

Seith, C.: Status und Schutz des extrakorporalen Embryos. Eine rechtsvergleichende Studie. Ethik und Recht in der Medizin 42/2. Nomos. Baden-Baden 2007 (377 S.).

Seith, C.: Die Statusfrage und der extrakorporale Embryo - ein rechtsvergleichender Blick über Ländergrenzen. In: Maio, G. (Hrsg.), Der Status des extrakorporalen Embryos - Perspektiven eines interdisziplinären Zugangs. frommann-holzboog. Stuttgart-Bad Cannstatt 2007, S. 463-527.

Seith, C., Eser, A., Koch, H.-G. (Hrsg.): Internationale Perspektiven zu Status und Schutz des extrakorporalen Embryos - International Perspectives on the Status and Protection of the Extracorporeal Embryo. Rechtliche Regelungen und Stand der Debatte im Ausland. Ethik und Recht in der Medizin 42/1. Nomos. Baden-Baden 2007 (500 S.).

\section{Sieber, Ulrich}

2006

Sieber, U.: Grenzen des Strafrechts. Strafrechtliche, kriminologische und kriminalpolitische Grundlagenfragen im Programm der Strafrechtlichen Forschungsabteilung am Freiburger Max-Planck-Institut für ausländisches und internationales Strafrecht. In: Albrecht, H.-J., Sieber, U. (Hrsg.), Perspektiven der strafrechtlichen Forschung. Amtswechsel am Freiburger Max-Planck-Institut für ausländisches und internationales Strafrecht 2004. Schriftenreihe des MaxPlanck-Instituts für ausländisches und internationales Strafrecht. Interdisziplinäre Forschungsberichte aus Strafrecht und Kriminologie I 12. Duncker \& Humblot. Berlin 2006, S. 35-79.

Sieber, U.: Strafrechtsvergleichung im Wandel. Aufgaben, Methoden und Theorieansätze der vergleichenden Strafrechtswissenschaft. In: Sieber, U., Albrecht, H.-J. (Hrsg.), Strafrecht und Kriminologie unter einem Dach. Schriftenreihe des Max-Planck-Instituts für ausländisches und internationales Strafrecht. Interdisziplinäre Forschungsberichte aus Strafrecht und Kriminologie I 14. Duncker \& Humblot. Berlin 2006, S. 78-130.

Sieber, U.: Das neue Projekt eines „Max-Planck-Informationssystems für Strafrechtsvergleichung“. In: Sieber, U., Albrecht, H.-J. (Hrsg.), Strafrecht und Kriminologie unter einem Dach. Schriftenreihe des Max-Planck-Instituts für ausländisches und internationales Strafrecht. Interdisziplinäre Forschungsberichte aus Strafrecht und Kriminologie I 14. Duncker \& Humblot. Berlin 2006, S. 131-160.

Sieber, U.: Cybercrime and Jurisdiction in Germany. The Present Situation and the Need for New Solutions. In: Koops, B.-J., Brenner, S. W. (Hrsg.), Cybercrime and Jurisdiction. IT \& Law 11. T.M.C. Asser Press. Leiden 2006, S. 183-210.

Sieber, U.: Compliance Programme zur Bekämpfung der Wirtschaftskriminalität. In: The Quarterly Review of Corporation Law and Society 4, 73-146 (2006).
Sieber, U.: Unificación, armonización y cooperación: buscando soluciones para los sistemas federales y supranacionales de la justicia penal. In: Instituto Nacional de Ciencias Penales (INACIPE), Max-Planck-Institut für ausländisches und internationales Strafrecht (MPI) (Hrsg.), Hacia la unificación del derecho penal. Logros y desafíos de la armonización y homologación legislativa en México y en el mundo. INACIPE. México 2006, S. 5-26.

Sieber, U.: International Cooperation against Terrorist Use of the Internet. In: Revue Internationale de Droit Pénal 77, 395-449 (2006).

Sieber, U.: Liability for On-line Data Bank Services, in Germany. In: Sieber, U., Managing \& Planning Organization of the High Council of Informatics (Hrsg.), Liability for On-line Data Bank Services. Information Technology Law Series, I.R.I. 3. Teheran 2006, S. 111-210.

Sieber, U., Albrecht, H.-J. (Hrsg.): Strafrecht und Kriminologie unter einem Dach. Kolloquium zum 90. Geburtstag von Professor Dr. Hans-Heinrich Jescheck am 10. Januar 2005. Schriftenreihe des Max-Planck-Instituts für ausländisches und internationales Strafrecht. Interdisziplinäre Forschungsberichte aus Strafrecht und Kriminologie I 14. Duncker \& Humblot. Berlin 2006 (202 S.).

Sieber, U., Albrecht, H.-J. (Hrsg.): Perspektiven der strafrechtlichen Forschung. Amtswechsel am Freiburger Max-Planck-Institut für ausländisches und internationales Strafrecht 2004. Schriftenreihe des Max-Planck-Instituts für ausländisches und internationales Strafrecht. Interdisziplinäre Forschungsberichte aus Strafrecht und Kriminologie I 12. Duncker \& Humblot. Berlin 2006 (79 S.).

Sieber, U., Eser, A., Arnold, J. (Hrsg.): Strafrecht in Reaktion auf Systemunrecht. Vergleichende Einblicke in Transitionsprozesse. Teilband 9: China (Richter). Schriftenreihe des Max-Planck-Instituts für ausländisches und internationales Strafrecht. Strafrechtliche Forschungsberichte S 82.9. Duncker \& Humblot. Berlin 2006 (267 S.).

Sieber, U., Eser, A., Arnold, J. (Hrsg.): Strafrecht in Reaktion auf Systemunrecht. Vergleichende Einblicke in Transitionsprozesse. Teilband 10: Südkorea (Cho/Holzapfl/Richter). Schriftenreihe des Max-Planck-Instituts für ausländisches und internationales Strafrecht. Strafrechtliche Forschungsberichte S 82.10. Duncker \& Humblot. Berlin 2006 (126 S.).

Sieber, U., Eser, A., Kreicker, H. (Hrsg.): Nationale Strafverfolgung völkerrechtlicher Verbrechen. National Prosecution of International Crimes. Teilband 7: Völkerstrafrecht im Ländervergleich (Kreicker). Schriftenreihe des Max-Planck-Instituts für ausländisches und internationales Strafrecht. Strafrechtliche Forschungsberichte S 95.7. Duncker \& Humblot. Berlin 2006 (424 S.).

Sieber, U., Koch, H.-G., Simon, J.-M.: Strafbare Mitwirkung von Führungspersonen in Straftätergruppen und Netzwerken: Eine rechtsvergleichende Analyse. In: MaxPlanck-Gesellschaft zur Förderung der Wissenschaften e. V. (Hrsg.), Jahrbuch 2006 der Max-Planck-Gesellschaft - CDROM-Anhang. Max-Planck-Gesellschaft zur Förderung der Wissenschaften e.V. München 2006, (4 S.).

Sieber, U., Managing \& Planning Organization of the High Council of Informatics (Hrsg.): Liability for Online Data Bank Services. Information Technology Law Series, I.R.I. 3. Teheran (2006) 398 S.

\section{7}

Sieber, U.: Grenzen des Strafrechts. Grundlagen und Herausforderungen des neuen strafrechtlichen Forschungsprogramms am Max-Planck-Institut für ausländisches und internationales Strafrecht. In: Zeitschrift für die Gesamte Strafrechtswissenschaft 119, 1-68 (2007).

Sieber, U.: Ceza Hukukunun Sinirlari. In: Yenisey, F. (Hrsg.), Ceza Hukukuna Giriş. Beta. Istanbul 2007, S. 173-228. 
Sieber, U., Albrecht, H.-J.: Stellungnahme zu dem Fragenkatalog des Bundesverfassungsgerichts in dem Verfahren 2 BvR 392/07 zu $\$ 173$ Abs. 2 S. 2 StGB - Beischla zwischen Geschwistern. 2007 (132 S.). http://www.mpicc. de/de/data/pdf/05-08-inzest_gutachten.pdf

Sieber, U., Brunst, P.W.: Cyberterrorism and other use of the Internet for terrorist purposes. Threat analysis and evaluation of international conventions. In: (Hrsg.), Cyberterrorism and other use of the Internet for terrorist purposes. Council of Europe. Straßburg, 2007, S. 10-105.

Sieber, U., Höfinger, F. M.: Allgemeine Grundsätze der Haftung, Kapitel 18.1. In: Hoeren, T., Sieber, U. (Hrsg.) Handbuch Multimedia-Recht, 18. Aufl. 2007. Verlag C.H Beck. München 2007, S. 1-72.

Sieber, U., Liesching, M.: Die Verantwortlichkeit der Suchmaschinenbetreiber nach dem Telemediengesetz. In Multimedia und Recht 10, 8, 1-30 (2007).

Sieber, U., Eser, A., Arnold, J. (Hrsg.): Strafrecht in Reaktion auf Systemunrecht. Vergleichende Einblicke in Transitionsprozesse. Teilband 11: Chile (Millaleo Hernández), Uruguay (Fernández). Schriftenreihe des Max-PlanckInstituts für ausländisches und internationales Strafrecht. Strafrechtliche Forschungsberichte S 82.11. Duncker \& Humblot. Berlin 2007 (643 S.)

Sieber, U., Hoeren, T. (Hrsg.): Handbuch MultimediaRecht. Grundwerk und Ergänzungslieferungen bis 2007. Verlag C.H. Beck. München 2007 (3380 S.).

\section{Silverman, Emily}

\section{6}

Silverman, E.: The Constitution and the Sentencing Process: Does Modern Presumptive Sentencing Respect Defendants' Rights? In: Berg, M., Kapsch, S., Streng, F. (Hrsg.), Criminal Justice in the United States and Germany: History, Modernization, and Reform. Universitätsverlag Winter. Heidelberg 2006, S. 87-104

Silverman, E.: Meiguo de xingshi peichang zhidu (shang). In: (Chinese) Criminal Law Review 10, 223-259 (2006).

\section{7}

Silverman, E.: Meiguo de xingshi peichang zhidu. In (Chinese) Criminal Law Review 11, 321-393 (2007).

\section{Simon, Jan-Michael}

2006

Simon, J.-M.: Guatemala. In: Arnold, J., Simon, J.-M., Woischnik, J. (Hrsg.), Estado de Derecho y Delincuencia de Estado en América Latina. Una visión comparativa. Universidad Nacional Autónoma de México. México 2006 S. 353-406.

Simon, J.-M.: Criminal Accountability and Reconciliation. In: Albrecht, H.-J., Simon, J.-M., Rezaei, H., Rohne, H.-C., Kiza, E. (Hrsg.) Conflicts and Conflict Resolution in Middle Eastern Societies - Between Tradition and Modernity. Schriftenreihe des Max-Planck-Instituts für ausländisches und internationales Strafrecht. Interdisziplinäre Forschungsberichte aus Strafrecht und Kriminologie I 13 Duncker \& Humblot. Berlin 2006, S. 99-116.

Simon, J.-M., Albrecht, H.-J., H. Rezaei, H.-C. Rohne, E. Kiza (Hrsg.): Conflicts and Conflict Resolution in Middle Eastern Societies - Between Tradition and Modernity. Schriftenreihe des Max-Planck-Instituts für ausländisches und internationales Strafrecht. Interdisziplinäre Forschungsberichte aus Strafrecht und Kriminologie I 13 Duncker \& Humblot. Berlin 2006 (660 S.).
Simon, J.-M., Arnold, J., Woischnik, J. (Hrsg.): Estado de Derecho y Delincuencia de Estado en América Latina. Una visión comparativa. Universidad Nacional Autónoma de México. México 2006 (420 S.).

\section{7}

La Función del Derecho Penal en Casos de Violencia Colectiva: Algunas Consideraciones sobre los Objetivos y Fines del Derecho Penal en Situaciones de Posconflicto. In: García Ramírez, Islas de González Mariscal: Panorama Internacional Sobre Justicia Penal. Séptimas Jornadas sobre Justicia Penal Instituto de Investigaciones Jurídicas de la UNAM. México D.F. 2007, S. 555-566.

\section{Son, Misuk}

2007

Son, M.: Die mittelbare Täterschaft und das System der Tatbeteiligung im koreanischen StGB. In: Zeitschrift für die Gesamte Strafrechtswissenschaft 119, 750-767 (2007).

\section{Spoenle, Jan}

2007

Spoenle, J.: Anmerkung zu OLG Karlsruhe, Beschluss vom 27.11.2006 - 3Ss 219/05. In: Juris Praxisreport itRecht, 1, Seq. No.: Anmerkung 7 (2007).

Spoenle, J.: Anmerkung zu AG Offenburg, Beschluss vom 20.07.2007 - 4 Gs 442/07. In: Juris Praxisreport itRecht, 8, Seq. No.: Anmerkung 6 (2007).

Spoenle, J.: Anmerkung zu LG Konstanz, Beschluss vom 27.10.2006 - 4 Qs 92/06. In: Juris Praxisreport itRecht, 12, Seq. No.: Anmerkung 2 (2007).

Spoenle, J., Ernst, S.: Weinversandhandel und Jugendschutz. In: Zeitschrift für das Gesamte Lebensmittelrecht 34, 1, 114-124 (2007).

\section{Tellenbach, Silvia}

2006

Tellenbach, S.: Bloodfeuds and How to Bring Them to an End - Experiences from Turkey. In: Albrecht, H.-J., Simon, J.-M., Rezaei, H., Rohne, H.-C., Kiza, E. (Hrsg.), Conflicts and Conflict Resolution in Middle Eastern Societies - Between Tradition and Modernity. Schriftenreihe des Max-Planck-Instituts für ausländisches und internationales Strafrecht. Interdisziplinäre Forschungsberichte aus Strafrecht und Kriminologie I 13. Duncker \& Humblot. Berlin 2006, 637-643.

Tellenbach, S: O novom Ugolovnom kodekce Turzii (Zakon No 5237 ot 26.09.2004). In: Kriminologija - Bčera - Segodnja-Zavtra 10, 1, 117-126 (2006).

Tellenbach, S.: O novom Ugolovnom kodekce Turzii. In: Ugolovnoe Pravo 6, 2, 7477 (2006).

Tellenbach, S.: Tagungsbericht: 30. Tagung der Gesellschaft für Rechtsvergleichung vom 22.-24. September 2005 in Würzburg. VI. Fachgruppe für vergleichende Rechtsgeschichte, orientalische Rechte und ethnologische Rechtsforschung. In: Juristenzeitung 61 (2006) 453-454.

\section{7}

Tellenbach, S.: Les Tribunaux militaires et Juridictions d'exception en Turquie. In: Lambert Abdelgawad, E. (Hrsg.), Juridictions militaires et Tribunaux d'exception en mutation. UMR de droit comparé. Université Paris 1 Panthéon-Sorbonne, CNRS. Paris 2007, S. 437-450. 
Tellenbach, S.: Zur Verfassung der Islamischen Republik Iran. Vorbilder - Wandlungen - Ergebnisse. In: Conermann, S., Schaffar, W. (Hrsg.), Die schwere Geburt von Staaten - Verfassungen und Rechtskulturen in modernen asiatischen Gesellschaften. Bonner Asienstudien. EB-Verlag. Schenefeld/Hamburg 2007, S. 369-386.

Tellenbach, S.: Roma Statüsü kabul edilmeli midir? Sözleşmeye taraf Olmayan Devletlerin Tereddütleri Uluslararası Ceza Divanı. In: Uluslararası Ceza Divanı. (Hrsg.) Yenisey, Feridun. Arıkan Verlag, Istanbul (2007) 71 (80)-79 (88).

Tellenbach, S.: Der Weg zum Islam - Weg ohne Umkehr? Zur Perspektive der Schari'a. In: Kadelbach, S. (Hrsg.), Die Freiheit der Religion im europäischen Verfassungsrecht. Parhisi, Parinas. Schriften zur Europäischen Integration und Internationalen Wirtschaftsordnung 9. Nomos. Baden-Baden 2007, S. 29-38.

Tellenbach, S.: Die Rolle der Ehre im Strafrecht in der Türkei, 619-662, Übersichtsbericht ausgewählte arabische Länder, 701-723, Rechtsvergleichende Zusammenfassung, 723-808. In: Tellenbach, S. (Hrsg.), Die Rolle der Ehre im Strafrecht. Schriftenreihe des Max-Planck-Instituts für ausländisches und internationales Strafrecht. Strafrechtliche Forschungsberichte S 111. Duncker \& Humblot. Berlin 2007.

Tellenbach, S. (Hrsg.): Die Rolle der Ehre im Strafrecht. Schriftenreihe des Max-Planck-Instituts für ausländisches und internationales Strafrecht. Strafrechtliche Forschungsberichte S 111 . Duncker \& Humblot. Berlin 2007 (813 S.).

Tellenbach, S., Scholler, H. (Hrsg.): Position und Aufgaben des Richters nach westlichem und nach islamischem Recht. Mohr Siebeck. Tübingen 2007 (127 S.).

\section{Tränkle, Stephanie} 2007

Tränkle, S.: Im Schatten des Strafrechts. Eine Untersuchung der Mediation in Strafsachen am Beispiel des deutschen Täter-Opfer-Ausgleichs und der französischen médiation pénale auf der Grundlage von Interaktions- und Kontextanalysen. Schriftenreihe des Max-Planck-Instituts für ausländisches und internationales Strafrecht. Kriminologische Forschungsberichte K 135. Duncker \& Humblot. Berlin 2007 (392 S.).

\section{von Dewitz, Clivia}

\section{6}

von Dewitz, C.: NS-Gedankengut und Strafrecht. Die $\$ \$ 86,86 a$ StGB und $\$ 130$ StGB zwischen der Abwehr neonazistischer Gefahren und symbolischem Strafrecht. Schriftenreihe des Max-Planck-Instituts für ausländisches und internationales Strafrecht. Strafrechtliche Forschungsberichte S 106. Duncker \& Humblot. Berlin 2006 (304 S.)

\section{Wade, Marianne} 2006

Wade, M.: The Power to Decide - Prosecutorial Control, Diversion and Punishment in European Criminal Justice Systems Today. In: Jehle, J.-M., Wade, M. (Hrsg.), Coping with Overloaded Criminal Justice Systems - The Rise of Prosecutorial Power. Springer. New York 2006, S. 27-126.

Wade, M., Jehle, J.-M. (Hrsg.): Coping with Overloaded Criminal Justice Systems - The Rise of Prosecutorial Power. Springer. New York 2006 (333 S.).
2007

Wade, M. (Hrsg.): Fear v. Freedom Post 9/11 - the European Perspective. In: European Journal on Criminal Policy and Research 13, 1-2 (2007)

Wade, M.: Fear vs Freedom Post 9/11 - A European Debate: Introduction. In: European Journal on Criminal Policy and Research 13, 3-12 (2007).

\section{Wahl, Thomas}

\section{6}

Wahl, T.: Datenschutz im Rahmen der polizeilichen Zusammenarbeit unter besonderer Berücksichtigung des SIS. In: Epiney, A., Theuerkauf, S. (Hrsg.), Datenschutz in Europa und die Schweiz - La protection des données en Europe et la Suisse. Schulthess. Zürich et al. 2006, S. 79-134.

\section{7}

Wahl, T.: European Union - Foundations - Community Powers in Criminal Matters, 7-11, European Union - Foundations - The Hague Programme Review, 11-12, European Union - Institutions - OLAF, 13-15, European Union - Institutions - Europol, 15-17, European Union Institutions - Eurojust, 17-18, European Union - Specific Areas of Crime/Substantive Criminal Law - Protection of Financial Interests, 19-21, European Union - Specific Areas of Crime/Substantive Criminal Law - Tax Fraud/ VAT, 22, European Union - Specific Areas of Crime/Substantive Criminal Law - Corruption, 22-23, European Union - Specific Areas of Crime/Substantive Criminal Law - Money Laundering, 23-24, European Union - Specific Areas of Crime/Substantive Criminal Law - Money Counterfeiting, 24-25, European Union - Specific Areas of Crime/Substantive Criminal Law - Counterfeiting and Piracy, 25-28, European Union - Specific Areas of Crime/ Substantive Criminal Law - Organised Crimef, 28-29, European Union - Specific Areas of Crime/Substantive Criminal Law - Cybercrime, 29, European Union - Specific Areas of Crime/Substantive Criminal Law - Other (Cybercrime, Illegal Employment, Racism and Xenophobia), 29-30, European Union - Procedural Criminal Law - Data Protection, 31-33, European Union - Procedural Criminal Law - Ne bis in idem, 33-34, European Union - Cooperation - Mutual Administrative Assistance, 35-36, European Union - Cooperation - Customs Cooperation and Police Cooperation, 36-38, European Union - Cooperation - European Arrest Warrant, 38-39, European Union - Cooperation - European Supervision Order/Transfer of Sentenced Person, 39-40, European Union - Cooperation - E-Justice and Exchange of Information on Criminal Records, 40-41, In: eucrim - the European Criminal Law Associations' Forum, 01-02, (2007).

Wahl, T.: European Union - Foundations - Community Powers in Criminal Matters, 76-78, European Union - Foundations - The Hague Programme Review, 78-79, European Union - Foundations - Legislation, 79-81, European Union - Institutions - OLAF, 82-83, European Union - Institutions - Europol, 83-84, European Union - Institutions - Eurojust/European Judicial Network, 84-86, European Union - Specific Areas of Crime/Substantive Criminal Law - Protection of Financial Interests, 87-91, European Union - Specific Areas of Crime/Substantive Criminal Law - Tax Fraud/VAT, 91-95, European Union - Specific Areas of Crime/Substantive Criminal Law - Cigarette Smuggling, 95-97, European Union - Specific Areas of Crime/Substantive Criminal Law - Counterfeiting and Piracy, 99, European Union - Procedural Criminal Law - Procedural Safeguards and Data Protection, 100-104, European Union - Procedural Criminal Law - Ne bis in idem and Victim Protection, 104-105, European Union - Cooperation - Law 
Enforcement, Customs and Police Cooperation, 108-110 In: eucrim - the European Criminal Law Associations' Forum, 03-04, 108-110 (2007).

Wahl, T., Löhr, C., Kiesel, S.: Reform of the European Union. In: eucrim - the European Criminal Law Associations' Forum, 01-02, 2-7 (2007).

\section{Weigend, Ewa}

2006

Weigend, E.: Evropeizacja ugolovnogo prava v svetle evropejskoj konstitucii. In: Politika i Obshchestvo (Politik und Gesellschaft), 2, 41-55 (2006).

\section{7}

Weigend, E.: Der Europäische Haftbefehl und die polnische Verfassung - jüngste Entwicklungen. In: Zeitschrift für die Gesamte Strafrechtswissenschaft 119, 459-469 (2007).

Weigend, E.: Der Europäische Haftbefehl und die polnische Verfassung. In: Tomášek, M. (Hrsg.), European Law and National Criminal Legislation. Univerzita Karlova, Práv nická fakulta. Ediční středisko. Praha 2007, S. 342-347.

Weigend, E.: Instytucja Rasterfahndung w niemieckim prawie karnym oraz w świetle orzeczenia niemieckiego $\mathrm{Fe}$ deralnego Trybunału Konstytucyjnego. In: Krajewski, K (Hrsg.), Nauki penalne wobec problemów współczesnej przestępczości. Księga jubileuszowa z okazij 70. rocznicy urodzin profesora Andrzeja Gaberle. Festschrift für Professor Andrzej Gaberle zum 70. Geburtstag. Wolters Kluwer Polska. Warszawa 2007, S. 295-307.
Weigend, E., Zielińska, E.: Die Rolle der Ehre im Strafrecht in Polen. In: Tellenbach, S. (Hrsg.), Die Rolle der Ehre im Strafrecht. Schriftenreihe des Max-Planck-Instituts für ausländisches und internationales Strafrecht. Strafrechtliche Forschungsberichte S 111. Duncker \& Humblot. Berlin 2007, S. 529-588.

\section{Wößner, Gunda \\ 2006}

Wößner, G.: Typisierung von Sexualstraftätern. Ein empirisches Modell zur Generierung typenspezifischer Behandlungsansätze. Schriftenreihe des Max-Planck-Instituts für ausländisches und internationales Strafrecht. Kriminologische Forschungsberichte K 132. Duncker \& Humblot. Berlin 2006 (271 S.).

Wößner, G.: Differentialdiagnostik bei Sexualstraftätern. In: Obergfell-Fuchs, J., Brandenstein, M. (Hrsg.), Nationale und internationale Entwicklungen in der Kriminologie - Festschrift für Helmut Kury zum 65. Geburtstag. Verlag für Polizeiwissenschaft. Frankfurt a.M. 2006, S. 581-597.

\section{Wollenschläger, Bernward} 2006

Wollenschläger, B.: Effektive staatliche Rückholoptionen bei gesellschaftlicher Schlechterfüllung. Studien zu Staat, Recht und Verwaltung 7. Nomos. Baden-Baden 2006 (246 S.) 


\section{B. Vorträge}

\section{Albrecht, Hans-Jörg \\ 2006}

Albrecht, H.-J.: „Key Lessons from Research on The Role of Defence Councils in Chinese Death Penalty Proceedings“. International Seminar "Strengthening the Defence in Death Penalty Cases", Chinese Academy of Social Sciences (CASS) in Zusammenarbeit mit dem Great Britain China Centre (GBCC). Minzu Hotel. Peking/VR China, 14.01.2006.

Albrecht, H.-J.: „Legal Culture, Terrorist Threats and Incapacitation“. Conference on Global Responses to Terrorism: The Context of State, Culture and Religion. National Defense College. Tel Aviv/Israel, 25.01.2006.

Albrecht, H.-J.: „Schuld und Strafe - Europäische Perspektiven“ und „Schuld und Strafe im Islamischen Rechtskreis“. Deutsche Richterakademie, "Schuld und Strafe“. Trier, 03.02.2006.

Albrecht, H.-J.: „Developments in the Fields of International Criminal Law and International Criminal Justice". Beijing Normal University, College of Criminal Sciences. Peking/VR China, 19.02.2006.

Albrecht, H.-J.: „German Criminal Procedural Law An Introduction“. University for Political Sciences and Law. Peking/VR China, 22.02.2006.

Albrecht, H.-J.: „Elektronische Fußfessel - Der Stand der Entwicklungen in Europa“. 21. Eickelborner Fachtagung, Westfälisches Zentrum für Forensische Psychiatrie. Lippstadt, 02.03.2006.

Albrecht, H.-J.: „Civilian Protection in Armed Conflicts: The Application of International Humanitarian Law in the XXI Century“. Universidad Alfonso X el Sabio, Madrid, 22.03.2006.

Albrecht, H.-J.: „Junge Immigranten und Gewalt“. Schlagwort Integration. Junge Zuwanderer, Lebenslagen, Gewalt: Probleme und Lösungsansätze im Vergleich. Friedrich Ebert Stiftung. Berlin, 23.03.2006.

Albrecht, H.-J.: „Litigation and Alcohol Policy“. Conference on The Alcohol Industry and Alcohol Policy. Schæffergården. Gentofte/Dänemark, 06.04.2006.

Albrecht, H.-J.: „Juvenile Justice Systems: a comparative approach“. University of Tehran, Faculty of Law. Teheran/Iran, 01.05.2006

Albrecht, H.-J.: „Crime Prevention: International and comparative perspectives“. Institute for Strategic Studies and Crime Prevention. Teheran/Iran, 01.05.2006.

Albrecht, H.-J.: „International Criminal Justice, Criminal Sanctions and Sentencing“ und „Legislation for Security: Post 9/11 Developments“. University of Qom, Law School. Ghom/Iran, 02.05.2006

Albrecht, H.-J.: „Victims of Terrorism Legislation“ und „Economic Criminal Law: Recent Developments“. University of Qom, Law School. Ghom/Iran, 03.05.2006.

Albrecht, H.-J.: „Changes in Concepts of Security“. University of Qom, Law School. Ghom/Iran, 04.05.2006.

Albrecht, H.-J.: „Terrorism, Security and Developments in Criminal Law“. University of Mazandaran. Saary/Iran, 06.05.2006.
Albrecht, H.-J.: „Death and Dying: Legal Implications“. Maxnet Aging: Conference III. University of Virgina. Charlottesville/USA, 10.05.2006.

Albrecht, H.-J.: „Corruption and Corruption Control“. Beijing Normal University, Law Faculty. Peking/VR China, 22.05.2006.

Albrecht, H.-J.: „Einführung in das deutsche Jugendstrafrecht“. University for Political Sciences and Law, Law Faculty. Peking/VR China, 24.05.2006.

Albrecht, H.-J.: „Das Spannungsfeld zwischen Pressefreiheit und Strafverfolgung". Juristischer Salon, Justizministerium Niedersachsen. Hannover, 30.05.2006.

Albrecht, H.-J.: „Proaktive Ermittlungsmethoden Theoretische und empirische Analysen zu Anwendung und Wirkungen“. Kriminalpolitisches Forum. Universität Bern. Bern/Schweiz, 12.06.2006.

Albrecht, H.-J.: „Discriminations Ethniques et la Police en Allemagne“. LEA Workshop: Discriminations, Police, Minorités Ethniques. Maison des Sciences de l'Homme. Paris/Frankreich, 27.06.2006

Albrecht, H.-J.: „Immigration, Law Enforcement and Criminal Justice“, Kriminalpolitik in High Crime Societies. Spenerhaus. Frankfurt a.M., 15.07.2006.

Albrecht, H.-J.: „Historical Developments of Torture: Social and cultural factors that have hindered the prevention of torture". Great Britain China Centre, Workshop „Cutting Torture in the People's Republic of China: from impunity to accountability". Renmin University. Peking/VR China, 12.08.2006.

Albrecht, H.-J.: „Public and Institutional Reactions to Terrorism and Their Impact on Society“. MPG Präsentation Forschungsperspektiven 7. Rahmenprogramm. Brüssel/Belgien, 11.09.2006.

Albrecht, H.-J.: „Die Rechtswirklichkeit der Hausdurchsuchung". Sitzung des Beirats Pro Justitia. St. LeonRot, 13.09.2006

Albrecht, H.-J.: „Risikoforschung und präventive Interventionen - Eine Zusammenfassung“. Landespräventionsrat NRW: „Prävention von Gewalt gegen alte Menschen“. Maternushaus. Köln, 16.09.2006

Albrecht, H.-J.: „Privatisierung des Maßregelvollzugs?“ Konferenz zum Maßregelvollzug, Verdi Bundesverwaltung. Berlin, 27.09.2006

Albrecht, H.-J.: „Korruption - Eine kriminologische Perspektive“. Tagung „Korruption und Korruptionsbekämpfung". Katholische Akademie. Trier, 12.10.2006.

Albrecht, H.-J.: „Drogenkonsum und Strafverfolgung“. Tagung „Suchtprävention“. Landeskriminalamt. Düsseldorf, 19.10.2006.

Albrecht, H.-J.: „Internationale Kriminalität, Gewaltökonomie und Menschenrechtsverbrechen“. Gewaltmärkte. Friedrich-Ebert-Stiftung. Bonn, 06.11.2006.

Albrecht, H.-J.: „Entwicklungen des Internationalen Strafrechts und der internationalen Strafjustiz“. Internationale Juristenkommission e. V.: Nürnberg: 60 Jahre danach. Beginn und Entwicklung der Internationalen Strafgerichtsbarkeit. Historischer Rathaussaal. Nürnberg, 11.11.2006. 
Albrecht, H.-J.: „Gewaltzyklen“. Freiburger Interventionsprojekt gegen häusliche Gewalt. Altes Kaufhaus. Freiburg i. Br., 16.11.2006.

Albrecht, H.-J.: „Gewaltzyklen“. Gewalt im sozialen Nahraum. Polizeiführungsakademie. Münster, 01.12.2006.

Albrecht, H.-J.: „Opening Address“. ARCA-Net International Conference: Conflicts, Conflict Resolution and Natural Resources. University of Tehran. Teheran/Iran 03.12 .2006

Albrecht, H.-J.: „Criminological Research at the Max Planck Institute for Foreign and International Crimina Law“. University of Tehran, Iranian Sociological Association. Teheran/Iran, 03.12.2006.

Albrecht, H.-J.: „Comparative Perspectives on Criminal Sanctions“. ARCA-Net International Conference: Conflicts, Conflict Resolution and Natural Resources. University of Tehran, Pardis Campus Qom. Ghom/Iran, 05.12.2006.

Albrecht, H.-J.: „Legislative Responses to International Terrorism“. University of Public Security Beijing. Peking/VR China, 11.12.2006.

Albrecht, H.-J.: „Police Education and Training -- Challenges in the Face of Current Social Problems“ und „Evaluation of Anti-Terrorist Policies in Germany“. University of Public Security Beijing: Prevention of Crime. Peking/VR China, 11./13.12.2006.

Albrecht, H.-J.: „Anti-Terrorist Legislation Past 9/11“. Beijing Normal University, Law Faculty. Peking/VR China, 12.12.2006

\section{7}

Albrecht, H.-J.: „International Trends in Anti-Money Laundering Policies“. University of Science and Technology Macao. Macao/VR China, 25.01.2007.

Albrecht, H.-J.: „Polizeiforschung in Deutschland“. Delegation türkischer Polizeirechtsexperten. Max-PlanckInstitut für ausländisches und internationales Strafrecht Freiburg i. Br., 13.02.2007.

Albrecht, H.-J.: „Introduction to Max Planck Institute for Foreign and International Criminal Law“ und „Public Opinion and the Death Penalty“. Irish Centre of Human Rights, Great Britain China Centre. Launch Seminar: „Moving the Debate Forward. The Death Penalty in China." University of Ireland. Galway/Irland, 07.03.2007.

Albrecht, H.-J.: „Die Überwachung der Telekommuni kation - Implementations- und Evaluationsforschungen". Portugiesisch-deutsches Kolloquium: Welche Zukunft hat das Strafprozessrecht? Universidade do Minho, Escola de Direito. Braga/Portugal, 24.03.2007.

Albrecht, H.-J.: „Einführung - Islamforschung am MPI Freiburg“. Islam Workshop. Max-Planck-Institut für Ethnologische Forschung. Halle, 27.03.2007.

Albrecht, H.-J.: „Rechtliche Grundlagen des Straf- und Maßregelvollzugs“. Deutsche Psychologen Akademie: „Forensische Psychologie“. Kolpinghaus. Köln, 29.03.2007.

Albrecht, H.-J.: ,An Introduction to the Fair Trial Problems in the Chinese Criminal Justice System“. EU-China Dialogue Seminar Human Rights: „The Rights to a Fair Trial and Labour Rights“. Deutsche Telekom AG. Berlin, 09.05.2007.

Albrecht, H.-J.: „Die Sanktionierung schwerer Straßenverkehrsdelikte im internationalen Vergleich“. Deutsche Universität der Polizei. Münster, 14.05.2007.

Albrecht, H.-J.: „Victims of Terrorism“. Hebrew Uni versity: Victimology and Human Rights - Between the Local and the Global. Jerusalem/Israel, 21.05.2007.

Albrecht, H.-J.: „Combatting Terrorism and Human Rights”; „New Developments in Economic Criminal Law“ und „Criminal Policy and Minority Protection in Europe“;
"Sentencing and Criminal Sanctions in Germany“. Shahid Beheshti University Tehran: Iran-German Criminal Law and Criminology Conference. Teheran/Iran, 28./29.05.2007.

Albrecht, H.-J.: „Corruption Control and The UNConvention Against Corruption“. University of Tehran, Pardis Campus Qom. Ghom/Iran, 30.05.2007.

Albrecht, H.-J.: „Anti-Terrorism Policies, Intelligence and Human Rights". Moffit University. Ghom/Iran, 30.05.2007

Albrecht, H.-J.: „Criminal Sanctions, Sentencing and Criminological Research“. University of Tarbiat Modarres. Teheran/Iran, 31.05.2007.

Albrecht, H.-J.: „Scientific Co-Operation between the Max Planck Institute Freiburg and Chinese Universities". Co-Reach Conference: Collaboration China Europe. Royal Society. London/England, 04.06.2007.

Albrecht, H.-J.: „Victimology in Europe“. Consejo Gen eral Del Poder Judicial; Panorama Actual Y Perspectivas de la Victimologia - La Victimologia y El Systema Penal. Consejo General Del Poder Judicial. Madrid/Spanien, 06.06.2007.

Albrecht, H.-J.: „Überwachung der Telekommunikation auf dem Prüfstand“. Yeditepe Universität. Istanbul/ Türkei, 13.06.2007

Albrecht, H.-J.: „Vorfeldermittlungen - Strafprozessuale und polizeirechtliche Entwicklungen“. Bahçesehir Universität. Istanbul/Türkei, 13.06.2007.

Albrecht, H.-J.: „Risk Assessment and Terrorism: An Introduction“. Turkish National Police: 2nd Conference on Democracy and Global Security. Conrad Hotel. Istanbul/ Türkei, 15.06.2007.

Albrecht, H.-J.: „Understanding Informal and Formal Systems of Justice and Conflict Resolution“. ARCA Net Workshop 2007: Northeast Asian Countries in Transition A Look into the Nature of Conflicts and the Importance of Social Values in China and Mongolia. Ulaanbataar/Mongolei, 02.07.2007.

Albrecht, H.-J.: „The Relationship Between Terrorist and Criminal Organizations and Society". Summer Academy on Human Security. Universität Graz, European Training and Research Center. Graz/Österreich, 19.07.2007.

Albrecht, H.-J.: „Criminal Policy and Criminal Law Reform - Political and Social Contexts". Brasilianisch-deutscher Workshop „Research on Crime, Criminal Law and Criminology“. Getulio Vargas Foundation und Max-PlanckInstitut für ausländisches und internationales Strafrecht in Zusammenarbeit mit dem Brazilian Center of Analysis and Planning. Harnack-Haus. Berlin, 24.07.2007.

Albrecht, H.-J.: „Ländergesetzgebung zum Jugendstrafvollzug: Analyse und Kritik, Thesen des Arbeitskreises 1" 27. Deutscher Jugendgerichtstag, Pädagogische Hochschule. Freiburg i. Br., 17.09.2007.

Albrecht, H.-J., Kania, H., Oberwittler, D.: „Attitudes towards Punishment in the European Union - Preliminary results from the EU-ICS“. Annual Conference of the European Society of Criminology. Bologna/Italien, 27.09.2007.

Albrecht, H.-J.: „DNA Based Criminal Investigation: Problems and Prospects“. 8th Annual Conference of the Asian Association on Police Studies. National Police University, Yong-in City. Seoul/Korea, 02.10.2007.

Albrecht, H.-J.: „Money Laundering Control Regimes“. Beijing Normal University, Law School. Peking/VR China, 05.10.2007

Albrecht, H.-J.: "The Role of Defence Councils in Criminal Proceedings". 2nd International Forum on Criminal Justice. Beijing Friendship Hotel. Peking/VR China, 09.10.2007.

Albrecht, H.-J.: „Electronically Monitored House Arrest - The Place of Electronic Monitoring in Systems of Sanc- 
tions and Coercive Measures, Its (Cost) Effectiveness and Impacts on Recidivism Rates" und „Impact of Forthcoming EU Initiatives - Mutual Recognition of Non-Custodial PreTrial Supervision Measures“. Expert Workshop: The Reform of the Romanian Code of Criminal Procedure. Justizministerium. Bukarest/Rumänien, 12.10.2007.

Albrecht, H.-J.: „Schuld und Schuldstrafrecht im europäischen Ausland“ und „Schuld im islamischen Rechtskreis“. Deutsche Richterakademie: Schuld und Strafe. Trier, 17.10.2007.

Albrecht, H.-J.: „Fragestellungen und Methoden grenzüberschreitender vergleichender Forschung“. Max-PlanckInstitut für ausländisches und internationales Strafrecht, Internationale Max Planck Research School für Strafrechtsvergleichung. Freiburg i. Br., 10.11.2007.

Albrecht, H.-J.: „Trafficking in Humans and Human Rights“ und zusammen mit Raymond Teske: „Asylum Seekers and Hate Crimes“. American Society of Criminology, Annual Meeting 2007. Marriot Hotel. Atlanta/USA, 15.11.2007.

Albrecht, H.-J.: „Neue Ermittlungsmethoden und neue Formen sozialer Kontrolle“. Westfälische WilhelmsUniversität Münster: Kriminologie im Übergang. Münster, 01.12.2007.

Albrecht, H.-J.: „Strafrecht und Strafe“; „Internationale Kriminalität, Menschenrechtsverbrechen und Gewaltökonomie“ und „Zusammenfassung und Ausblick“. Max-Planck-Institut für ausländisches und internationales Strafrecht, Botschaft der Bundesrepublik Deutschland und Rechtswissenschaftliche Fakulät der Universität Buenos Aires: Deutsch-Argentinisches Strafrechtssymposium. Buenos Aires/Argentinien, 14./15.12.2007.

\section{Arnold, Jörg}

2006

Arnold, J.: „Entwicklungslinien des Feindstrafrechts in 5 Thesen“. Strafverteidigertag. Frankfurt a.M., 25.03.2006.

Arnold, J.: „Feindstrafrecht und Kriminologie“. Institut für Sozialwissenschaften und Kriminologie an der Universität Hamburg. Hamburg, 18.05.2006.

\section{7}

Arnold, J.: Laudatio zur Festschriftübergabe an Prof. Dr. Erich Buchholz anlässlich dessen 80. Geburtstag. Berlin, 13.02.2007.

Arnold, J.: „Vorschläge zur Verbesserung des Schutzes des Strafverteidigers: Auf dem Weg zu einem ,europäischen Strafverteidiger?" Tagung des Rates der Vorstände der Europäischen Rechtsanwaltskammern, Europaparlament. Straßburg/Frankreich, 12.10.2007.

\section{Brandenstein, Martin}

2006

Brandenstein, M.: „Hasskriminalität: Auswirkungen von Hafterfahrungen auf fremdenfeindliche jugendliche Gewalttäter“. Vortrag für Studierende der Katholischen Fachhochschule Freiburg. MPI für ausländisches und internationales Strafrecht. Freiburg i. Br., 18.10.2006

Brandenstein, M.: „The Effects of Imprisonment in the Context of Sentencing". ESC-Conference. Tübingen, 27.08.2006.

Brandenstein, M.: „Gewalt in unserer Gesellschaft Diskussion der Entwicklung und Folgen“. 11. Deutscher Präventionstag. Nürnberg, 09.05.2006

\section{7}

Brandenstein, M.: „Identity as Explanatory Concept for Hate Crime - First Results of an Empirical Study. "ESCConference. Bologna, 29.09.2007.

Brandenstein, M.: „Violent Xenophobic Juvenile Offenders in Prison - First Results of a Study and Implications for Rehabilitation Measures". Präsentation für Sozialpädagogen aus Kopenhagen/Dänemark. Max-Planck-Institut für ausländisches und internationales Strafrecht, Freiburg i. Br., 16.04.2007

\section{Brunst, Phillip W.}

2006

Brunst, Ph.: „Anonymität im Internet“. Vortrag im Rahmen des Doktorandenforums des Max-Planck-Instituts. Max-Planck-Institut für ausländisches und internationales Strafrecht. Freiburg i. Br., 30.05.2006.

Brunst, Ph.: „Hacker, Cracker, Bauernfänger. Strafrecht im Zeitalter des Internet“. Schulvortrag anlässlich der Jahreshauptversammlung der Max-Planck-Gesellschaft am Heinrich-von-Gagern-Gymnasium. Frankfurt a.M., 11.07.2006.

Brunst, Ph.: „Encryption Technology and Methods to Provide Anonymous Communication“. Expertenvortrag auf Einladung des Europarates im Rahmen des Programms PACO Serbien. Belgrad/Serbien, 28.08.2006.

Brunst, Ph.: „Investigating Illegal Access - Social Engineering Techniques and Hacking Tools“. Belgrad/Serbien, 29.08.2006.

\section{7}

Brunst, Ph.: „New Investigation Instruments that Require the Cooperation of ISP“. Vortrag auf Einladung des Europarates. Belgrad/Serbien, 23.02.2007.

Brunst, Ph.: „Anonymous Communication - Benefit for the Society vs. Tool for Criminal Activities". Vortrag auf Einladung des Europarates. Belgrad/Serbien, 23.02.2007.

Brunst, Ph.: ,Anonymität und Ermittlungen im Internet“. Vortrag im Rahmen der Summer School der Strafrechtlichen Abteilung des Max-Planck-Instituts für ausländisches und internationales Strafrecht zum Thema „Aktuelle Kriminalitätsentwicklung, Internationales Strafrecht und Strafrechtsvergleichung. Forschungsfragen an den Grenzen des Strafrechts". Pécs/Ungarn, 17.06.2007.

Brunst, Ph.: „Hacker, Cracker und Bauernfänger. Strafrecht und das Internet“. Schulvorträge anlässlich der Jahreshauptversammlung der Max-Planck-Gesellschaft an der Käthe-Kollwitz-, Heinrich-Heine- sowie Bismarck-Schule. Kiel, 26./27./28.06.2007.

Brunst, Ph.: „Straftaten im Internet“. Vortrag an der katholischen Studentenverbindung Ripuaria. Freiburg i. Br., 05.07.2007

Brunst, Ph.: „Hacker, Cracker und Bauernfänger. Strafrecht und das Internet". Schulvorträge im Rahmen der Ersten Freiburger Schulvorträge an der Max-Weber-, der Richard-Fehrenbach-Schule sowie am Rotteck-Gymnasium. Freiburg i. Br., 16./17./18.07.2007.

Brunst, Ph.: „Complex Crimes: On the Phenomena of Cyber Crime“. Vortrag im Rahmen des deutsch-brasilianischen Workshops „Research on Crime, Criminal Law and Criminology“. Berlin, 24.07.2007.

Brunst, Ph.: „Technische Umsetzung einer OnlineÜberwachung“. Vortrag im Rahmen der Sitzung des Fachausschusses Strafrecht der Deutschen Gesellschaft für Recht und Informatik (DGRI). Freiburg, 28.09.2007.

Brunst, Ph.: „Use of the Internet for Terrorist Purposes and Cyberterrorism“ und „Types of Cyberterrorist Attacks“. 
Vorträge auf Einladung der NATO im Advanced Research Workshop (ARW) „Responses to Cyber Terrorism“ im Centre of Excellence - Defense Against Terrorism (COE-DAT) Ankara/Türkei, 04.10.2007.

Brunst, Ph.: „Cyberterrorism - A Threat for Modern Society?" Präsentation für Mitarbeiter von Polizei und Steuerfahndung aus Tanzania im Rahmen der forensischen Ausbildung im 4n6-Programm. Freiburg i. Br., 09.10.2007.

Brunst, Ph.: „Cyberterrorismus - Eine neue Bedrohung aus dem Internet?” und „Die Cybercrime Konvention und der Rahmenbeschluss ,Angriffe auf Informationssysteme - aktuelle internationale Instrumente zur Bekämpfung de Computerkriminalität". Vorträge an der Deutschen Richterakademie in Wustrau im Rahmen der Tagung „Erscheinungsformen und Bekämpfungsstrategien der Internetkriminalität". Wustrau, 18.10.2007.

\section{Cornils, Karin}

2006

Cornils, K.: „National Criminalization of Internationa Crimes”. Helsinki/Finnland, 02.06.2006.

Cornils, K.: „Säkringsförvaring I Tyskland“ („Sicherungsverwahrung in Deutschland“). Dänische Strafrechtslehrertagung 2006. Hillrød/Dänemark, 24.08.2006.

\section{7}

Cornils, K.: „Comparative Criminal Law Research and International Harmonization“. Nordisk workshop för straffrätt. Rovaniemi/Finnland, 23.03.2007.

Cornils, K.: „Localizing Internet Crimes“. Gastvortrag Universität Rovaniemi. Rovaniemi/Finnland, 26.03.2007.

Cornils, K.: „Strafrecht der nordischen Länder: Einführung und aktuelle Forschungsfragen zur nordischen Zusammenarbeit in Strafsachen“. Vortrag im Rahmen de Summer School der Strafrechtlichen Abteilung des MaxPlanck-Instituts für ausländisches und internationale Strafrecht zum Thema „Aktuelle Kriminalitätsentwicklung, Internationales Strafrecht und Strafrechtsvergleichung. Forschungsfragen an den Grenzen des Strafrechts“. Pécs Ungarn, 19.06.2007.

\section{Engelhart, Marc}

2006

Engelhart, M.: „60 Jahre und mehr: Was ist aus dem Vermächtnis von Nürnberg geworden? Das Völkerstrafrecht gestern und heute“. Schulvortrag anlässlich der Jahreshauptversammlung der Max-Planck-Gesellschaft am Heinrichvon Gagern-Gymnasium. Frankfurt a.M., 11.07.2006.

\section{7}

Engelhart, M.: „Compliance-Programme zur Bekämpfung der Wirtschaftskriminalität in den USA“. Vortrag im Rahmen der Summer School der Strafrechtlichen Abteilung des Max-Planck-Instituts für ausländisches und internationales Strafrecht zum Thema „Aktuelle Kriminalitätsentwicklung, Internationales Strafrecht und Strafrechtsvergleichung. Forschungsfragen an den Grenzen des Strafrechts“. Pécs/Ungarn, 19.06.2007.

Engelhart, M.: „Der internationale Strafgerichtshof Strafverfolgungen im Namen der Menschheit". Schulvorträge im Rahmen der Ersten Freiburger Schulvorträge an der Max-Weber-, der Richard-Fehrenbach-Schule sowie am Rotteck-Gymnasium. Freiburg i. Br., 18.07.2007.

Engelhart, M.: „Die Sanktionierung von Unternehmen und Compliance-Programme“. Vortrag im Rahmen der Internationalen Max Planck Research School für Strafrechtsvergleichung. Freiburg i. Br., 11.12.2007.

\section{Eser, Albin}

2006

Eser, A.: „Von Nürnberg bis Den Haag. Zur Entwicklung der internationalen Strafjustiz bei Völkermord und Kriegsverbrechen“. Universität Konstanz, Seminar zur Rechtsentwicklung. Konstanz, 30.01.2006.

Eser, A.: „Überblick über die Grundlagen des Embryonenschutz- und des Stammzellgesetzes“. Symposium des Science Office und der ScheringStiftung über „Das Stammzellgesetz anhand konkreter Beispiele“. Berlin, 27.03.2006

Eser, A.: „Legal Issues of Stem Cell Research in German and Transnational Perspective". Rolduc Workshop 2006 on „Perspectives and Potentials of Embryonic Stem Cell Research“. Rolduc/Niederlande, 01.04.2006.

Eser, A.: „Introduction into the Structure and Jurisprudence of the International Criminal Tribunal for the Former Yugoslavia“. Visit of ELSA (European Law Students Association) of Muenster at the ICTY. Den Haag/Niederlande, 24.05.2006

Eser, A.: „Die UN als Hüterin des Völkerrechts? Der Internationale Strafgerichtshof zwischen Prävention, Versöhnung und Bestrafung“. Vortragsreihe „A More Secure and Better World?" - 60 Jahre Vereinte Nationen im Lichte Globaler Herausforderungen. Carl-Schurz-Haus/DeutschAmerikanisches Institut. Freiburg i. Br., 29.05.2006.

Eser, A.: „Challenges of International Criminal Justice“. International Colloquium on „Criminal Theory in Transition“. Helsinki/Finnland, 02.06.2006.

Eser, A.: „Criminality of Organisations - Lessons from Domestic Law: A Comparative Perspective“. Conference on "System Criminality in International Law". Center for International Law. Amsterdam/Niederlande, 20.10.2006.

Eser, A.: „The Nature and Rationale of Punishment“. International Conference on „The Grammar of Criminal Law. American, European, International." Benjamin Cardozo School of Law. New York City/USA, 05.11.2006.

Eser, A.: „Developments in International Criminal Justice. Reflections of a Judge at the ICTY“. Harvard Law School Faculty Club. Cambridge/Massachusetts/USA, 07.11.2006.

Eser, A.: „Humanitätsverbrechen und die Internationale Strafjustiz: Erfahrungen eines Richters am Internationalen Strafgerichtshof für das ehemalige Jugoslawien." Vortrag vor der K.D.St.V. Ripuaria. Freiburg i. Br., 12.12.2006.

2007

Eser, A.: „Protection of Human Rights by International Criminal Law“. „Dialogue on Human Rights - Dialog zwischen Juristen aus Freiburg und Isfahan“. Kommission für Menschenrechte des Vereins der Richter und Staatsanwälte und des Anwaltvereins Freiburg. Gerichtslaube. Freiburg i. Br., 26.03.2007.

Eser, A.: „Conflicts of Common Law and Civil Law in International Criminal Justice“. ICTY-Konferenz Graz über „The ICTY and Human Rights: Towards a Fair Trial in International Criminal Procedure?" Graz/Österreich, 29.03.2007.

Eser, A.: „Schwangerschaftsabbruch zwischen Lebensschutz und Selbstbestimmung. Rechtspolitische Erkenntnisse aus einem internationalen Rechtsvergleich". International Congress on „Justice and Human Values in Europe“. Karlsruhe, 10.05.2007.

Eser, A.: „Rechtskulturen im Wettstreit - Erfahrungen aus der internationalen Strafjustiz“. Festvortrag auf dem 95. Stiftungsfest der K.D.St.V. Falkenstein. Freiburg i. Br., 26.05.2007.

Eser, A.: „Challenges of International Criminal Justice: Lessons from The Hague“. Max-Planck-Institut für ausländisches und internationales Strafrecht. Freiburg i. Br. 13.11 .2007 
Eser, A.: „Reflexionen zur Prozessstruktur der internationalen Strafgerichtsbarkeit“. Dienstag-Seminar der Frankfurter Strafrechtslehrer. Frankfurt a.M., 18.12.2007.

\section{Forster, Susanne} 2007

Forster, S.: „Eingriffe in die Bewegungsfreiheit durch die Terrorismusgesetzgebung des Vereinigten Königreichs“. Kolloquium der Max Planck International Research School for Comparative Criminal Law (IMPRS). Max-PlanckInstitut für ausländisches und internationales Strafrecht. Freiburg i. Br., 17.04.2007.

\section{Galain Palermo, Pablo} 2006

Galain Palermo, P.: „El Estatuto de Roma y la Corte Penal Internacional“, Unterricht für Postgraduierte an der Universität von Trujillo. Trujillo/Peru, 22.03.2006.

Galain Palermo, P.: „Problemas de extradición y de entrega de sospechosos ante la Corte Penal Internacional". Katholische Universität. Lima/Peru, 24.03.2006.

Galain Palermo, P.: „Retaliation, mediation and punishment as methods for prevention and solution of violent crimes in Latin America“. Humboldt-Universität. Berlin, 06.09.2006.

Galain Palermo, P.: „Suspensão provisional do processo penal e terceira vía“. Colóquio Luso-Alemão, Simpósio de Direito Processual Penal. Homenagem ao Professor Doutor Figueiredo Dias, Universidad do Minho. Braga/Portugal, 21.03.2007.

Galain Palermo, P.: „Reparação como equivalente funcionãl da pena“. „Restaurative Justice: an analysis of brasilian experiences“. Fundación Getulio Vargas. San Pablo/ Brasilien, 17.08.2007.

Galain Palermo, P.: „Suspensão provisional del processo penal e alternatividade“. Universidad de Curitiba. Paranál Brasilien, 20.08.2007.

Galain Palermo, P.: „Suspensión provisional del proceso penal y alternatividad penal“. Reforma del derecho procesal penal uruguayo, Universidad de Montevideo. Montevideo/ Uruguay, 24.08.2007.

Galain Palermo, P.: „Reparación penal y fines de la pena“. Institut für Kriminologie und Wirtschaftsstrafrecht. Freiburg i. Br., 06.10.2007.

Galain Palermo, P.: „Reforming the system of criminal law in Uruguay: Present state of basic problems in the reform projects". Max-Planck-Institut für ausländisches und internationales Strafrecht. Freiburg i. Br., 15.10.2007.

Galain Palermo, P.: „Criminal reparation as a functional equivalent of punishment: A proposal set between two models of justice“. „New theoretical system of criminal law in the age of globalization". China University of Political Science and Law. Beijing/ VR China, 28.10.2007.

\section{Gröseling, Nadine}

Gröseling, N.: „Extraterritoriale Rechtsanwendung bei der Verfolgung von Straftaten im Internet“. Vortrag im Rahmen der Summer School der Strafrechtlichen Abteilung des Max-Planck-Instituts für ausländisches und internationales
Strafrecht zum Thema „Aktuelle Kriminalitätsentwicklung, Internationales Strafrecht und Strafrechtsvergleichung. Forschungsfragen an den Grenzen des Strafrechts“. Pécs/ Ungarn, 17.06.2007.

Gröseling, N.: „Das juristische Verfahren um den heimlichen Zugriff auf Computerdaten“. Vortrag im Rahmen der Sondersitzung des Fachausschusses Strafrecht der Deutschen Gesellschaft für Recht und Informatik (DGRI). Freiburg i. Br., 28.09.2007.

Gröseling, N.: „Extraterritoriale Rechtsanwendung bei der Verfolgung von Straftaten im Internet“. Vortrag vor einer Besuchergruppe der Universität Turku/Finnland. Freiburg i. Br., 18.10.2007.

\section{Höfinger, Frank Michael} 2006

Höfinger, F. M.: „Computerkriminalität und ihre strafrechtliche Bewältigung“. Vortrag im Rahmen von „FRITSI verfolgt: Aktuelle Fragen und Gesetzesvorhaben in der Computerkriminalität" der Freiburger IT Sicherheits-Initiative (FRITSI). Freiburg i. Br., 11.10.2006.

\section{7}

Höfinger, F. M., Nolde, M.: „Freedom of Speech and Extraterritorial Jurisdiction on the Internet“. „Dialogue on Human Rights - Dialog zwischen Juristen aus Freiburg und Isfahan“. Kommission für Menschenrechte des Vereins der Richter und Staatsanwälte und des Anwaltvereins Freiburg. Max-Planck-Institut für ausländisches und internationales Strafrecht. Freiburg i. Br., 29.03.2007.

Höfinger, F. M.: „Die europäische Harmonisierung des Computerstrafrechts und ihre Umsetzung in Deutschland“. Vortrag im Rahmen der Summer School der Strafrechtlichen Abteilung des Max-Planck-Instituts für ausländisches und internationales Strafrecht zum Thema „Aktuelle Kriminalitätsentwicklung, Internationales Strafrecht und Strafrechtsvergleichung. Forschungsfragen an den Grenzen des Strafrechts“. Pécs/Ungarn, 18.06.2007.

\section{Huber, Barbara} 2007

Huber, B.: „Por fin entre nosotros! Negociaciones en el proceso penal alemán“. Deutsch-Argentinisches Symposium. Buenos Aires/Argentinien, 14.12.2007.

\section{Jähnke, Jochen}

\section{7}

Jähnke, J.: „Zusammenarbeit in der MPG: Ein Erfahrungsbericht aus dem CMS-Projekt von fünf juristischen Instituten“. Treffen der DV-Mitarbeiter der MPG. Jena, 07.11 .2007

\section{Jarvers, Konstanze}

\section{7}

Jarvers, K.: „Strafrecht in Italien: Einführung und aktuelle Forschungsfragen zur Beschleunigung des Strafverfahrens". Vortrag im Rahmen der Summer School der Strafrechtlichen Abteilung des Max-Planck-Instituts für ausländisches und internationales Strafrecht zum Thema „Aktuelle Kriminalitätsentwicklung, Internationales Strafrecht und Strafrechtsvergleichung. Forschungsfragen an den Grenzen des Strafrechts“. Pécs/Ungarn, 18.06.2007. 


\section{Kania, Harald}

2007

Kania, H., Albrecht, H.-J., Oberwittler, D.: „Attitudes towards Punishment in the European Union - Preliminary results from the EU-ICS“. Annual Conference of the European Society of Criminology. Bologna/Italien, 27.09.2007.

\section{Kieselmann, René}

2007

Kieselmann, R.: „Standortermittlung - Möglichkeiten, Notwendigkeiten, Technik und Recht“. Stiftungstagung de Alcatel-Lucent Stiftung für Kommunikationsforschung zum Thema „Mobilität und Kontext - Zukunftsentwicklungen der mobilen Kommunikation in Recht und Technik“. Stuttgart, 11.05.2007.

\section{Kilchling, Michael 2006}

Kilchling, M.: „Gegenwärtige Probleme und Weiterentwicklung europäischer Strafgesetzgebung am Beispie von OK und Terrorismus". Schule für Verfassungsschutz. Swisttal-Heimerzheim, 02.03./10.05.2006.

Kilchling, M.: „Geldwäsche versus Terrorismusfinanzierung“; „Neuere Entwickungen in der Opferforschung und „Gesetzliche Regelungen zugunsten von Opfern von Terrorismus in international vergleichender Perspektive“ Vorträge im Rahmen der Summer School der Kriminologischen Abteilung des Max-Planck-Instituts für ausländisches und internationales Strafrecht zum Thema „Neuere Entwicklungen in der kriminologischen Theorie und kriminologischen Forschung". Pécs/Ungarn, 19./20./22.06.2006.

Kilchling, M.: „Criminal Justice Systems in Europe“. Max-Planck-Institut für ausländisches und internationales Strafrecht. Freiburg i. Br., 14.08.2006.

Kilchling, M.: „Support for Victims of Terrorism in the Context of National and International Counter-Terrorism Policies“. 6th Annual Conference of the European Society of Criminology. Tübingen, 28.08.2006.

Kilchling, M.: „Die Praxis der Vermögensabschöpfung im internationalen Vergleich“. Arbeitstagung des Hessischen Ministeriums der Justiz „Vermögensabschöpfung und Rechtsstaatlichkeit“. Bad Salzhausen, 30.08.2006.

Kilchling, M.: „Introduction to victimology“ und „Victims of terrorism“. HUMSEC - International Summer Academy on Human Security, special focus on the connection between transnational terrorist and criminal organizations in the Western Balkan region. Graz/Österreich, 04.09.2006.

Kilchling, M.: „Organisierte Umweltkriminalität als wachsender Markt? Tendenzen, Entwicklung und juristische Strategien der Bekämpfung in Europa“. Studientagung der Katholischen Akademie Trier für Polizeibeamte, Richter, Staatsanwälte und Interessierte über „Globales Wirtschaften: Wirtschaft ohne Moral?“. Trier, 11.10.2006.

Kilchling, M.: „Gefährdete Berufsgruppen - Anwälte, Notare, Steuerberater und Wirtschaftsprüfer“. 4. Jahreskongress zur Geldwäschebekämpfung 2006. BankersCampus. Potsdam, 21.10.2006.

\section{7}

Kilchling, M.: „The Legal Framework for Victim Participation in Criminal Proceedings in Germany, Canada, and the US: Victim Impact Statement versus Nebenklage“. International workshop: Victim Participation in Justice and Therapeutic Jurisprudence: A Comparative Analysis. International Institute for the Sociology of Law (IISL). Oñati/ Spanien, 08.03.2007.
Kilchling, M.: „Entwicklung des rechtlichen Instrumentariums zum Zugriff auf die Gewinne der OK in Europa“. Katholische Akademie. Trier, 04.05.2007.

Kilchling, M.: „Gegenwärtige Probleme und Weiterentwicklung europäischer Strafgesetzgebung am Beispiel von OK und Terrorismus“. Schule für Verfassungsschutz. Swisttal-Heimerzheim, 06.07./16.08.2007.

Kilchling, M.: „European Experiences with Restorative Justice“. und „Money-Laundering Control and Forfeiture in Europe“. Brasilianisch-deutscher Workshop „Research on Crime, Criminal Law and Criminology“. Getulio Vargas Foundation und Max-Planck-Institut für ausländisches und internationales Strafrecht in Zusammenarbeit mit dem Brazilian Center of Analysis and Planning. Harnack-Haus. Berlin, 24.07.2007.

Kilchling, M.: „Die Praxis der Vermögensabschöpfung im internationalen Vergleich“. Arbeitstagung des Hessischen Ministeriums der Justiz. Bad Salzhausen, 19.09.2007.

Kilchling, M.: „Die Durchsuchung von Wohn- und Geschäftsräumen“. Stiftung ProJustitia. St. Leon-Rot, 15.10.2007.

Kilchling, M.: „Opferschutz und der Strafanspruch des Staates - ein Widerspruch?" Deutsche Richterakademie Wustrau. Wustrau, 29.10.2007.

Kilchling, M.: „Das Opfer in Strafrechtstheorie und -praxis“. Johannes Kepler Universität. Linz/Österreich, 03.12.2007.

\section{Knust, Nandor} 2006

Knust, N.: „Transitional Justice in the Middle East: Lessons learned from Sierra Leone, East Timor and Cambodia“. Conference on Conflicts, Conflict Resolution and Natural Resources in the Middle East. Universität von Tehran/Campus Qom. Qom/Iran, 06.12.2006.

\section{7}

Knust, N.: „Strafrecht und Gacaca: Die Aufarbeitung des ruandischen Völkermords“. International Max Planck Research School for Comparative Criminal Law. MaxPlanck-Institut für ausländisches und internationales Strafrecht. Freiburg i. Br., 15.05.2007.

Knust, N.: „Völkerstrafrecht: Einführung und aktuelle Forschungsfragen zur Ahndung des Völkermords in Ruanda“. Vortrag im Rahmen der Summer School der Strafrechtlichen Abteilung des Max-Planck-Instituts für ausländisches und internationales Strafrecht zum Thema ,Aktuelle Kriminalitätsentwicklung, Internationales Strafrecht und Strafrechtsvergleichung. Forschungsfragen an den Grenzen des Strafrechts“. Pécs/Ungarn, 20.06.2007.

Knust, N.: „Aspects of Transitional Justice: Justice in Afghanistan“. Independent Afghan Human Rights Commission. Kabul/Afghanistan, 18.08.2007.

Knust, N.: „Reforming criminal procedure in Rwanda: Neo-traditional procedures to cope with human rights violations and procedural human rights guarantees“. Vortrag im Rahmen des Workshops „Reforming Criminal Justice 12: Key Issues of Contemporary Criminal Procedure" und des Besuches einer Expertendelegation des Rule of Law Program der Konrad-Adenauer-Stiftung. Freiburg i. Br., 15.10.2007.

Knust, N.: „Internationale, nationale und (neo-)traditionelle strafrechtliche Aufarbeitung von Massengewalt am Fallbeispiel von Ruanda“. Vortrag anlässlich der Delegation von Wissenschaftlern aus Finnland. Max-Planck-Institut für ausländisches und internationales Strafrecht. Freiburg i. Br., 18.10.2007. 
Knust, N.: „The Max Planck Institute for Foreign and International Criminal Law: An African Perspective in the Institutionalization of International Criminal Law". Workshop: „The Role of Africa in the Institutionalization of International Criminal Law“. Addis Abeba/Äthiopien, 17.12.2007.

\section{Koch, Hans-Georg} 2006

Koch, H.-G.: „Strafbarer und straffreier Schwangerschaftsabbruch nach dem SFHG“. Seminar „Grundlagen der Sozial- und Konfliktberatung bei Schwangerschaft". Pro Familia Bundesverband/Bundeszentrale für gesundheitliche Aufklärung. Frankfurt a.M./Eppenhain (Taunus), 17.03.2006/22.09.2006

Koch, H.-G.: „Auslandsbezüge des Stammzellgesetzes“. Schering Science Office. Berlin, 27.03.2006.

Koch, H.-G.: „Strafbare Mitwirkung von Führungspersonen in Straftätergruppen und Netzwerken - Eine rechtsvergleichende Analyse“. Fachbeirats- und Kuratoriumssitzung am Max-Planck-Institut für ausländisches und internationales Strafrecht. Freiburg i. Br., 27.04.2006.

Koch, H.-G.: „Aufgaben und Verpflichtung der EthikKommission aus der Sicht des Rechts“. Jubiläumskolloquium „25 Jahre Ethik-Kommission im Klinikum - 10 Jahre öffentlich-rechtliche Ethik-Kommission der Albert-Ludwigs-Universität Freiburg“. Freiburg i. Br., 11.10.2006.

Koch, H.-G.: „Combating Counterfeit Medicines: Preliminary Thoughts on a Legal Comparative Project“. WHO/ IMPACT 1st General Meeting. Bonn, 14.11.2006.

Koch, H.-G.: „Reproduktionsmedizin: Der Status des Embryos". Symposium der Deutschen Akademie der Naturforscher Leopoldina. Lübeck, 17.11.2006.

\section{7}

Koch, H.-G.: „Strafbarer und straffreier Schwangerschaftsabbruch nach dem SFHG“. Seminar „Grundlagen der Sozial- und Konfliktberatung bei Schwangerschaft". Pro Familia Bundesverband/Bundeszentrale für gesundheitliche Aufklärung. Frankfurt a.M./Eppenhain (Taunus), $09.03 .2007 / 28.09 .2007$

Koch, H.-G.: „Expertenanhörung im Bundesministerium für Gesundheit zu Fragen der Arzneimittelfälschung“. Berlin, 20.04.2007.

Koch, H.-G.: „Rechtsfragen aus der Praxis der Ethikkommission“. Nachwuchsgruppen-Expertenhearing am MaxPlanck-Institut für ausländisches öffentliches Recht und Völkerrecht. Heidelberg, 31.05.2007.

Koch, H.-G.: „Haftungsrechtliche Aspekte der Beratungstätigkeit und der Patientenautonomie." 25. Freiburger Workshop „Ethische und psychologische Grundlagen genetischer Beratung“. Freiburg i. Br., 02.06.2007.

Koch, H.-G.: „Lernen am toten Körper aus rechtlicher Sicht." Klinikum der Johann Wolfgang Goethe-Universität. Frankfurt a.M., 20.06.2007.

Koch, H.-G.: „Fortpflanzungstourismus als Argument?“ 2. Göttinger Workshop im Medizinrecht „Umwege zum eigenen Kind: Verfügbarkeit donogener reproduktionsmedizinischer Techniken“. Georg-August-Universität. Göttingen, 22.06.2007.

Koch, H.-G.: „Begutachtung von potentiellen Lebendspendern durch Ethik-Kommissionen." Klausurwoche „Körperkommerzialisierung des Interfakultativen Zentrums für Ethik in den Wissenschaften Tübingen. Heidelberg, 25.07.2007.
Koch, H.-G.: „Konsequenzen für den Arzt aus dem Urteil des Bundesverfassungsgerichts zum SchwangerenhilfeErgänzungsgesetz“. Bayerische Landesärztekammer. München, 06.10.2007.

Koch, H.-G.: „Developing Effective Legislation to Combat Counterfeit Medical Products: Comparative study on concepts of criminal legislation“. WHO/IMPACT 2nd General Meeting. Lissabon/Portugal, 10.12.2007.

\section{Köllisch, Tilman 2006}

Köllisch, T.: „An external validation of male adolescents' self-reported delinquency." 6th Annual Conference of the European Society of Criminology. Tübingen, 29.08.06.

\section{7}

Köllisch, T., Oberwittler D.: „Wie dunkel ist das Dunkelfeld? Befragungen zur selbstberichteten Delinquenz von Jugendlichen." Vorlesung im Rahmen der Methodenausbildung im Grundstudium Soziologie, Universität Freiburg. Freiburg i. Br., 15.01.2007.

Köllisch, T.: „Konsequenzen des demographischen Wandels für die Kriminalisierung jugendlichen Problemverhaltens.“ Tagung „Soziodemografischer Wandel - Soziale und kulturelle Konsequenzen für Jugendliche“ der Sektion Jugendsoziologie der DGS. Potsdam, 01.03.07.

Köllisch, T.: „Abweichendes Verhalten - Ausgrenzung - soziale Kontrolle. Grundlegende Erkenntnisse für die Soziale Arbeit vor Ort." Hochschule Esslingen. Esslingen, 04.06.07.

Köllisch, T.: „Nimmt die Kriminalität von Jugendlichen in Deutschland immer weiter zu?" Schulvorträge anlässlich der Jahreshauptversammlung der Max-Planck-Gesellschaft am Gymnasium Kaltenkirchen und der BismarckschuleElmshorn, 26./27.06.2007.

Köllisch, T.: „Die heroingestützte Behandlung Opiatabhängiger - auch ein Erfolgsmodell zur Reduktion von Gewaltdelikten?" 10. Wissenschaftliche Fachtagung der Neuen Kriminologischen Gesellschaft (NKG) „Drogen Sucht - Kriminalität“. Medizinische Universität. Innsbruck/ Österreich, 21.09.07.

\section{Kouassi, Adome Blaise}

2006

Kouassi, A.B.: „Quo Vadis Afrika? - Konflikte und Friedenskonsolidierung auf dem Krisenkontinent“. AlbertLudwigs-Universität. Freiburg i.Br, 25.01.2006

Kouassi, A. B.: „Rechtssysteme in Afrika südlich der Sahara“. Max-Planck-Institut für ausländisches und internationales Strafrecht. Freiburg i. Br./Institut für Afrikanistik, Universität zu Köln. Freiburg i. Br./Köln, 01.02./13.05.2006.

Kouassi, A. B.: „Stereotypen und Vorurteile über Afrika: Mittel und Wege zu einem Wandel westlicher Vorurteile gegenüber Afrika und den Afrikanern“. Droste-HülshoffGymnasium. Freiburg i. Br., 31.07.2006.

Kouassi, A. B.: „Das soziale Sicherheitssystem in Afrika südlich der Sahara, am Beispiel der Frankophonen Länder“. Jugendherberge. Heidelberg, 10.12.2006.

\section{7}

Kouassi, A. B.: „Migration zwischen Chance und Verlust: Afrika braucht Stabilität - Rechtsstaatlichkeit und Entwicklung“. Oberhaugstett, 03.02.2007. 


\section{Lafrenz, Bianca}

\section{7}

Lafrenz, B.: „Psychological Analysis of Familial Homicide-Suicide“. First Meeting of the Working Group „Familia Homicide-Suicide in European Countries“. Max-PlanckInstitut für ausländisches und internationales Strafrecht Freiburg i. Br., 08.03.2007.

Lafrenz, B.: „Tötungsdelikte in der Familie - Erklärungsversuche und Risikofaktoren“. Vortrag im Doktorandenforum des Max-Planck-Instituts für ausländisches und internationales Strafrecht. Freiburg i. Br., 03.05.2007.

Lafrenz, B.: „Familialer Homizid-Suizid“. Vortrag im Rahmen der institutsinternen „Brown-Bag“-Veranstaltungs reihe des Max-Planck-Instituts für ausländisches und internationales Strafrecht. Freiburg i. Br., 22.05.2007.

Lafrenz, B.: „Wahrheit und Lüge bei Zeugenaussagen - Die Beurteilung der Glaubhaftigkeit“. Schulvortrag an der Freien Waldorfschule Freiburg-Rieselfeld. Freiburg i. Br. 04.07.2007.

Lafrenz, B., Oberwittler, D.: „Familial Homicide-Suicide in Germany". Annual Conference of the European Society of Criminology. Bologna/Italien, 27.09.2007.

\section{Macke, Julia}

\section{7}

Macke, J., Spaniol, Margret: „The Influence of the Jurisprudence of the German Federal Constitutional Court on Criminal Procedure“. „Dialogue on Human Rights - Dialog zwischen Juristen aus Freiburg und Isfahan“. Kommission für Menschenrechte des Vereins der Richter und Staatsanwälte und des Anwaltvereins Freiburg. Gerichtslaube. Frei burg i. Br., 26.03.2007.

Macke, J.: „Der Sicherheitsrat der Vereinten Nationen als Strafgesetzgeber“. International Max Planck Research School für Strafrechtsvergleichung. Max-Planck-Institut fü ausländisches und internationales Strafrecht. Freiburg i. Br. 11.12 .2007

\section{Manso Porto, Teresa}

\section{7}

Manso Porto, T.: „Courts and uniformity of the crimi nal/judicial process in the European Union“. Leitung des Workshops „Universalitätsprinzip, Territorialitätsprinzip und völkerrechtliche Gerichtsbarkeit". Spanische Richterschule. Barcelona/Spanien, 18.04.2007.

Manso Porto, T.: „Courts and uniformity of the criminal/judicial process in the European Union“. Leitung des Workshops „Einfluss der europäischen Gerichtsbarkeit au die Entwicklung des materiellen Strafrechts und des Strafverfahrensrechts der Mitgliedstaaten“. Spanische Richterschule. Barcelona/Spanien, 19.04.2007.

Manso Porto, T.: „La lucha contra el blanqueo de capitales en el marco internacional y europeo: resultados desde una perspectiva de derecho comparado“ („Die Bekämpfung der Geldwäsche im internationalen und europäischen Raum Ergebnisse aus rechtsvergleichender Sicht"). Las Nuevas Tendencias Delictivas en el Siglo XXI. Valle de Pedro González, Estado Nueva Esparta/Venezuela, 29.05.2007.

Manso Porto, T.: „Strafrecht in Spanien: Einführung und aktuelle Forschungsfragen zum Terrorismus“. Vortrag im Rahmen der Summer School der Strafrechtlichen Abteilung des Max-Planck-Instituts für ausländisches und internationales Strafrecht zum Thema „Aktuelle Kriminalitätsentwicklung, Internationales Strafrecht und Strafrechtsvergleichung. Forschungsfragen an den Grenzen des Strafrechts“. Pécs/Ungarn, 18.06.2007.

\section{Nolde, Malaika \\ 2006}

Nolde, M.: „Bekämpfung der Internet-Kriminalität in Deutschland und Rumänien“. Deutsch-Rumänische Strafrechtstagung. Bukarest/Rumänien, 25.03.2006.

\section{7}

Nolde, M., Höfinger, F. M.: „Freedom of Speech and Extraterritorial Jurisdiction on the Internet“. „Dialogue on Human Rights - Dialog zwischen Juristen aus Freiburg und Isfahan“. Kommission für Menschenrechte des Vereins der Richter und Staatsanwälte und des Anwaltvereins Freiburg. Max-Planck-Institut für ausländisches und internationales Strafrecht. Freiburg i. Br., 29.03.2007.

Nolde, M.: „Nationale Schutzstrategien im globalen Cyberspace“. Vortrag im Rahmen der Summer School der Strafrechtlichen Abteilung des Max-Planck-Instituts für ausländisches und internationales Strafrecht zum Thema „Aktuelle Kriminalitätsentwicklung, Internationales Strafrecht und Strafrechtsvergleichung. Forschungsfragen an den Grenzen des Strafrechts“. Pécs/Ungarn, 18.06.2007.

Nolde, M., Schnabel, C.: „Verfassungsrechtliche Grenzen der staatlichen Inhaltskontrolle im Internet". Herbstakademie der Deutschen Stiftung für Recht und Informatik. Potsdam, 15.09.2007.

\section{Oberwittler, Dietrich 2006}

Oberwittler, D.: „Why Neighbourhoods OR schools are not enough: Lessons from a multilevel analysis of contextual effects on juvenile delinquency". Presentation at the Netherlands Institute for the Study of Crime and Law Enforcement (NSCR). Leiden/Niederlande, 17.03.2006.

Oberwittler, D.: „The impact of ethnic and social segregation on children and adolescents. Results of a German multilevel study“. Workshop Ethnic Segregation in Germany and Europe, Social Science Research Centre (WZB) Berlin, 31.03.2006.

Oberwittler, D.: „Soziale Schichten oder Sozialmilieus? Zwischenstand einer Kontroverse“. Habilitationsvortrag. Universität Bielefeld. Bielefeld, 5.07.2006.

Oberwittler, D., Ceccato, V.: „Comparing spatial patterns of robbery evidence from a Western and an Eastern European city". 6th Annual Conference of the European Society of Criminology. Tübingen, 26.08.2006.

Oberwittler, D., Pauwels, L.: „Involvement in violen youth groups by gender and ethnicity. Findings from a cross national comparison between two urban samples in Belgium and Germany". 6th Annual Conference of the European Society of Criminology. Tübingen, 27.08.2006

Oberwittler, D., Köllisch, T.: „An external validation of male adolescents' self-reported delinquency“. 6th Annual Conference of the European Society of Criminology. Tübingen, 28.08.2006.

Oberwittler, D., Wikström, P.-O.: „How small can you go? A community study using very small spatial units". Workshop Units of Analysis in the Study of Crime at Place, Netherlands Institute for the Study of Crime and Law Enforcement (NSCR). Leiden/Niederlande, 06.09.2006.

Oberwittler, D., Wikström, P.-O.: „Behavioural Contexts and Victimization". Annual Meeting of the American Society of Criminology. Los Angeles/USA, 01.11.2006.

\section{7}

Oberwittler D., Köllisch, T.: „Wie dunkel ist das Dunkelfeld? Befragungen zur selbstberichteten Delinquenz von 
Jugendlichen." Vorlesung im Rahmen der Methodenausbildung im Grundstudium Soziologie, Universität Freiburg. Freiburg i. Br., 15.01.2007.

Oberwittler, D.: „Familial Homicide-Suicide in European Countries - The macro-sociological dimension“. First Workshop on Homicide-Suicide in Europe, Max-PlanckInstitut für ausländisches und internationales Strafrecht. Freiburg i. Br., 08.03.2007.

Oberwittler, D.: „Sind Stadtviertel und Schulen an der Gewaltproduktion beteiligt? Erkenntnisse über sozialräumliche Kontexteffekte“. DFG-Graduiertenkolleg „Gruppenbezogene Menschenfeindlichkeit". Universität Bielefeld. Bielefeld, 17.04.2007.

Oberwittler, D.: „Attitudes to the Death Penalty and the Sources of Punitiveness among Chinese and U.S. American Students - Preliminary Findings“. Workshop „Public Opinion and the Death Penalty in China“. Max-Planck-Institut für ausländisches und interntionales Strafrecht. Freiburg i. Br., 05.05.2007.

Oberwittler, D., Albrecht, H.-J., Kania, H.: „Attitudes towards Punishment in the European Union - Preliminary results from the EU-ICS“. Annual Conference of the European Society of Criminology. Bologna/Italien, 27.09.2007.

Oberwittler, D., Lafrenz, B.: „Familial Homicide-Suicide in Germany". Annual Conference of the European Society of Criminology. Bologna/Italien, 27.09.2007.

Oberwittler, D., Qi, S.: „Attitudes towards the Death Penalty among Chinese, U.S. American and German Students in Germany". Annual Conference of the European Society of Criminology. Bologna/Italien, 28.09.2007.

\section{Özsöz, Figen} 2006

Özsöz, F.: „Rechtsextreme Jugendgewalt: Erscheinungsformen und Erklärungsansätze“. Schulvorträge anlässlich der Jahreshauptversammlung der Max-Planck-Gesellschaft an der Weidigschule Butzbach sowie an der Feldbergschule Oberursel. Butzbach/Oberursel, 11./12.07.2006.

Özsöz, F.: „Hasskriminalität: Auswirkungen von Hafterfahrungen auf fremdenfeindliche jugendliche Gewalttäter". Vortrag für Studierende der Katholischen Fachhochschule Freiburg. Max-Planck-Institut für ausländisches und internationales Strafrecht. Freiburg i. Br., 18.10.2006.

\section{7}

Özsöz, F.: „Rechtsextremistische Gewalttäter im Jugendstrafvollzug: Verfestigung oder Ablösung von rechtsextremistischen Orientierungen im Haftverlauf". Konferenz „Der Aufstand der Zuständigen - Was kann der Rechtsstaat gegen Rechtsextremismus tun?" Friedrich-Ebert-Stiftung. Berlin, 20.03.2007.

Özsöz, F.: „The Impact of Imprisonment on Juvenile Hate-Crime Offenders in a Longitudinal Perspective“. First ENRIC-Meeting on Life Course Criminology. Leiden/Niederlande, 31.03.2007

Özsöz, F.: „Der Umgang mit rechtsextremistischen Gewalttätern im Strafvollzug". Vortrag im Rahmen der institutsinternen „Brown-Bag“-Veranstaltungsreihe des MaxPlanck-Instituts für ausländisches und internationales Strafrecht. Freiburg i. Br., 12.06.2007.

Özsöz, F.: „Rechtsextreme Jugendgewalt: Erscheinungsformen und Erklärungsansätze“. Schulvortrag an der Freien Waldorfschule Freiburg-Rieselfeld. Freiburg i. Br., 04.07.2007.

Özsöz, F.: „Rechtsextreme Jugendgewalt“. Powerpointvorführung auf der Wissenschaftsmeile der Albert-LudwigsUniversität. Freiburg i. Br., 1 1.07.2007.
Özsöz, F.: „Skinheads Behind Bars - How does prison affect the development of juvenile hate crime offenders?" 7th Annual Conference of the European Society of Criminology. Bologna/Italy, 29.09.2007.

\section{Quenzer, Carolin}

\section{6}

Quenzer, C.: „Sexualdelinquenz - Rückfälligkeit - Prognose“. Vortrag am Max-Planck-Institut für ausländisches und internationales Strafrecht. Freiburg i. Br., 23.11.2006.

\section{7}

Quenzer, C.: „Psychologie und Kriminologie“. Schulvortrag anlässlich der Jahreshauptversammlung der MaxPlanck-Gesellschaft am Gymnasium Wellingdorf. Kiel, 26.06.2007.

Quenzer, C.: ,Jugendliche und heranwachsende Sexualstraftäter - eine empirische Studie über Rückfälligkeit und Risikofaktoren“. Institut für Forensische Psychiatrie der Charité - Universitätsmedizin Berlin. Berlin, 16.07.2007.

Quenzer, C.: ,Juvenile Sex Offenders. A unique group?" Max-Planck-Institut für ausländisches und internationales Strafrecht. Freiburg i. Br., 18.09.2007.

Quenzer, C.: „Jugendliche und heranwachsende Gewalt- und Sexualstraftäter - erste Ergebnisse einer empirischen Studie“. Fachgruppentagung Rechtspsychologie der Deutschen Gesellschaft für Psychologie. Kiel, 20.09.2007.

Quenzer, C.: „Prognose bei (jungen) Sexualstraftätern Kann man Rückfälligkeit vorhersagen?" Ruprecht-Karls-Universität, Institut für Kriminologie. Heidelberg, 08.10.2007.

Quenzer, C.: „Kriminalität und insbesondere Sexualdelinquenz unter Berücksichtigung des Geschlechterproporz“. Zonta Club. Berlin, 30.10.2007.

Quenzer, C.: „Junge Sexualstraftäter - eine empirische Studie über Rückfälligkeit und Risikofaktoren“. AlbertLudwigs-Universität, Internationale Graduiertenakademie. Freiburg i. Br., 06.11.2007.

Quenzer, C.: „Sexualdelinquenz - Neue Ergebnisse aus der Forschung". Kriminalistisch-Kriminologische Forschungsstelle der Hessischen Polizei beim Landeskriminalamt. Wiesbaden, 12.11.2007.

Quenzer, C.: „Anforderungen an Prognosegutachten“. Max-Planck-Institut für ausländisches und internationales Strafrecht. Freiburg i. Br., 30.11.2007.

Quenzer, C.: „Sexualdelinquenz und Rückfälligkeit“. Rotary Club. Biedenkopf, 10.12.2007.

\section{Qi, Shenghui}

\section{7}

Qi, S., Oberwittler, D.: „Attitudes towards the Death Penalty among Chinese, U.S. American and German Students in Germany". Annual Conference of the European Society of Criminology. Bologna/Italien, 28.09.2007.

\section{Rabbat, Paul J.}

2006

Rabbat, P.J.: „Self-Defence under International Law and the Legality of the War in Iraq". Schulvortrag anlässlich der Jahreshauptversammlung der Max-Planck-Gesellschaft am Heinrich-von-Gagern-Gymnasium. Frankfurt a.M., 11.07.2006. 
Rabbat, P. J.: ,Judging Saddam Hussein: The Iraqi High Tribunal and the Dujail Case“. Max-Planck-Institut für ausländisches und internationales Strafrecht. Freiburg i. Br. 29.11.2006

\section{Rinceanu, Johanna}

\section{6}

Rinceanu, J.: „Deutsches Strafprozessrecht für Ausländer“. Max-Planck-Institut für ausländisches und internationales Strafrecht. Freiburg i. Br., 28.11.2006.

\section{7}

Rinceanu, J.: „Courts and uniformity of the criminal/ judicial process in the European Union“. Leitung des Workshops „Discussion of experiences on the principle of universal justice, the principle of territoriality in relation to the international courts". Spanische Richterschule. Barcelona/ Spanien, 18.04.2007.

Rinceanu, J.: „Courts and uniformity of the criminal/ judicial process in the European Union“. Leitung des Workshops „Discussion of experiences on the influence of European courts on the development of criminal and procedura law of Member States". Spanische Richterschule. Barcelona/Spanien, 19.04.2007.

Rinceanu, J.: „Strafrecht in Deutschland: Einführung und aktuelle Forschungsfragen zu Absprachen im Strafprozess“. Vortrag im Rahmen der Summer School der Strafrechtlichen Abteilung des Max-Planck-Instituts für ausländisches und internationales Strafrecht zum Thema „Aktuelle Kriminalitätsentwicklung, Internationales Strafrecht und Strafrechtsvergleichung. Forschungsfragen an den Grenzen des Strafrechts“. Pécs/Ungarn, 19.06.2007.

\section{Sadr Touhid-Khaneh, Mohammad 2007}

Sadr Touhid-Khaneh, M.: „Afghanistan: Das Verhältnis zwischen staatlichem Recht und Sharia im Aufbau de Strafjustiz“. Islamworkshop. Max-Planck-Institut für ethnologische Forschung. Halle/Saale, 27.03.2007.

\section{Sieber, Ulrich}

\section{6}

Sieber, U.: „Verantwortlichkeit von Suchmaschinenbetreibern für rechtswidrige Inhalte im Internet“. Berlin, 15.02.2006.

Sieber, U.: „Mutual Recognition of Judicial Decision and the Framework Decision on the European Evidence Warrant". OLAF-Kolloquium „Protection of the Financia Interests of the European Union of 25: New Challenges, Old Obstacles“. Danzig/Polen, 18.03.2006.

Sieber, U.: „New Ways of Information Exchange between the Associations for European Criminal Law“. Meeting of the Presidents of the Associations for European Criminal Law. Danzig/Polen, 19.03.2006.

Sieber, U.: „Die Forschungen am Max-Planck-Institut für ausländisches und internationales Strafrecht". Kolloquium mit einer Delegation von Richtern des Tribunal Supremo aus Madrid. Freiburg i. Br., 15.05.2006.

Sieber, U.: „Tasks, Methods and Theory of Comparative Criminal Law“. International Colloquium „Crimina Law Theory in Transition“, University of Helsinki, Department of Criminal Law and Judicial Procedure. Helsinki/ Finnland, 02.06.2006.
Sieber, U.: „The European Arrest Warrant“. Introduction to and Moderation of the Discussion on Basic Research Questions. Conference European Arrest Warrant: The Reality of a Vision. NH Leeuwenhorst - Noordwijkerhout, T. M.C. Asser Instituut. Den Haag/Niederlande, 16.06.2006.

Sieber, U.: „Identification of Lacunae in International Law and Action against Terrorism“. 10th meeting of the Committee of Experts on Terrorism (CODEXTER). Council of Europe. Straßburg/Frankreich, 21.06.2006.

Sieber, U.: „Haftung von Suchmaschinenanbietern Aktuelle Rechtlage und Reformfragen“. Konferenz „Selbstkontrolle Suchmaschinen: Suchmaschinen in der modernen Kommunikation“. Berlin, 21.09.2006.

Sieber, U.: „Rechtsrahmen betreffend die zwischenstaatliche Kooperation im Kampf gegen den Terrorismus (Strafrecht, Polizeirecht u.a.)“. Deutsch-amerikanisches Kolloquium „Rechtsfragen im Kampf gegen den Terrorismus“, Auswärtiges Amt und Max-Planck-Institut für ausländisches öffentliches Recht und Völkerrecht Heidelberg. Berlin, 12.10.2006.

Sieber, U.: „Use of the Internet for Terrorist Purposes and Cyberterrorism". 11th Meeting of the Committee of Experts on Terrorism (CODEXTER). Council of Europe. Straßburg/Frankreich, 06.12.2006.

\section{7}

Sieber, U.: „Das Max-Planck-Institut für ausländisches und internationales Strafrecht und das Institutsprojekt ,Rethinking European Criminal Justice“. Einführung zum internationalen Seminar „Rethinking European Criminal Justice?", Max-Planck-Institut für ausländisches und internationales Strafrecht. Freiburg i. Br., 20.01.2007.

Sieber, U.: „Verantwortlichkeit für Suchmaschinen nach dem TDG“. Gesprächskreis „Informationsrecht“ des Instituts für Öffentliches Recht der Universität Freiburg. Freiburg i. Br., 25.01.2007.

Sieber, U.: „Globalisierung, transnationale Kriminalität und Perspektiven des Europäischen Strafrechts“. Portugiesisch-deutsches Kolloquium „Welche Zukunft hat das Strafprozessrecht?" zu Ehren von Prof. Doutor Jorge de Figueiredo Dias zum 20-jährigem Jubiläum der Strafprozessordnung, Universidade do Minho, Escola de Direito. Braga/ Portugal, 21.03.2007.

Sieber, U.: „Aktuelle Forschungen des Max-PlanckInstituts für ausländisches und internationales Strafrecht". „Dialogue on Human Rights - Dialog zwischen Juristen aus "Freiburg und Isfahan“. Kommission für Menschenrechte des Vereins der Richter und Staatsanwälte und des Anwaltvereins Freiburg. Gerichtslaube. Freiburg i. Br., 29.03.2007.

Sieber, U.: „Les chemins de l'harmonisation-The Forces Driving Change“. Tagung „Les chemins de l'harmonisation“, Universität Neapel. Neapel/Italien, 12.04.2007.

Sieber, U.: „Cyberterrorism“. 12th meeting of the Committee of Experts on Terrorism (CODEXTER). Council of Europe. Straßburg/Frankreich, 24.04.2007.

Sieber, U.: „Conclusions for the Future of European Criminal Law“. Seminar "Re-launching the European project: needs for protecting EC interests and new strategies for penal integration pending the European Constitution", OLAF und Centro di Diritto Penale Europeo. Universität Catania. Catania/Italien, 26.05.2007.

Sieber, U.: „Aktuelle Veränderungen des Strafrechts in der Weltrisikogesellschaft“ und „Aufgaben, Methoden und Theorie der Strafrechtsvergleichung". Vorträge im Rahmen der Summer School der Strafrechtlichen Abteilung des Max-Planck-Instituts für ausländisches und internationales Strafrecht zum Thema „Aktuelle Kriminalitätsentwicklung, Internationales Strafrecht und Strafrechtsvergleichung. Forschungsfragen an den Grenzen des Strafrechts. Pécs/ Ungarn, 17.06.2007. 
Sieber, U.: „Private Law Making and Private Law Enforcement - The Challenges for Criminal Law". Präsentation zur Exzellenzinitiative der Rechtswissenschaftlichen Fakultät der Universität Freiburg zur Beantragung einer Graduiertenschule vor Vertretern des Gutachtergremiums der Deutschen Forschungsgemeinschaft. Bad Honnef, 20.07.2007.

Sieber, U.: „The Max Planck Institute for Foreign and International Criminal Law and its Research Agenda" und „International Taxonomy for Comparative Criminal Law. The Max Planck Information System for Comparative Criminal Law“. Brasilianisch-deutscher Workshop „Research on Crime, Criminal Law and Criminology“. Getulio Vargas Foundation und Max-Planck-Institut für ausländisches und internationales Strafrecht in Zusammenarbeit mit dem Brazilian Center of Analysis and Planning. Harnack-Haus. Berlin, 24.07.2007.

Sieber, U.: „The Max Planck Society, the Max Planck Institute for Foreign and International Criminal Law and its Research Programme“. Vortragsveranstaltung für Studenten des amerikanischen Stetson College of Law. Freiburg i. Br., 26.07.2007.

Sieber, U.: „The New Threats of the Global Risk Society and their Challenges to Traditional Criminal Law". XVth International Congress of Social Defense über „Criminal Law between War and Peace: Justice and Cooperation in Criminal Matters in International Military Interventions". Universität Toledo. Toledo/USA, 22.09.2007.

Sieber, U.: „Der heimliche Zugriff auf Computerdaten im Rahmen strafrechtlicher Ermittlungen“. Tagung der Deutschen Gesellschaft für Recht und Informatik (DGRI). Freiburg i. Br., 28.09.2007.

Sieber, U.: „Mastering Complexity in Global Cyberspace: The Harmonization of Computer-Related Criminal Law". Beijing Normal University. Peking/VR China, 05.10.2007.

Sieber, U.: „European Criminal Law“. International Symposium der China University of Political Science and Law über „European Union Law and European Integration“. Peking/VR China, 07.10.2007.

Sieber, U.: „Online-Durchsuchungen“. Gutachtliche Stellungnahme für das Bundesverfassungsgericht zur Online-Durchsuchung. Karlsruhe, 10.10.2007.

Sieber, U.: „Aufgaben und Forschungsprojekte des Max-Planck-Instituts für ausländisches und internationales Strafrecht". Einführungsvortrag anlässlich eines Besuchs von Juristen aus den weltweiten Rechtsstaatsprogrammen der Konrad-Adenauer-Stiftung in der Bundesrepublik Deutschland. Freiburg i. Br., 15.10.2007.

Sieber, U.: „Online-Searches in the Global Cyberspace: A New Threat for Criminals and for Civil Liberties". Internationales Seminar „Computer Crimes and Cybercrimes: Global Offences, Global Answers“. Universität Verona. Verona/Italien, 27.10.2007.

Sieber, U.: „Aufgaben, Methoden und Theorie der Strafrechtsvergleichung“. Kompaktseminar der Max Planck International Research School for Comparative Law. MaxPlanck-Institut für ausländisches und internationales Strafrecht. Freiburg i. Br., 09./10.11.2007.

Sieber, U.: „Models and Solutions for the Future European Criminal Law". OLAF International Scientific Conference „Law Enforcement Authorities and the Protection of the Financial Interests of the European Community“. European Law Research Association. Warschau/Polen, 15./16.1 1.2007.

Sieber, U.: „Kriminalitätsbekämpfung im Spannungsfeld zwischen Sicherheit und Freiheit". Herbsttagung des Bundeskriminalamts für Vertreter von Sicherheitsbehörden, Politik und Wissenschaft „Tatort Internet - eine globale Herausforderung für die Innere Sicherheit“. Wiesbaden, 22.11.2007.
Sieber, U.: „Die strafrechtliche Zusammenarbeit zwischen dem Max-Planck-Institut für ausländisches und internationales Strafrecht und der argentinischen Strafrechtswissenschaft“ (Eröffnungsrede) und „Transnationale Kriminalität und Modelle eines transnationalen Strafrechts“. Deutsch-Argentinisches Symposium „Die Gegenwart der Kriminalität, der Strafrechtsentwicklung und Strafrechtskritik“. Universität Buenos Aires, Departamento de Derecho Penal y Criminología. Buenos Aires, 13.12.2007.

Sieber, U.: „Strafrecht und technischer Fortschritt“. Deutsch-Argentinisches Symposium „Die Gegenwart der Kriminalität, der Strafrechtsentwicklung und Strafrechtskritik“, Universität Buenos Aires, Departamento de Derecho Penal y Criminología. Buenos Aires/Argentinien, 15.12.2007.

\section{Silverman, Emily}

2006

Silverman, E.: „September 11, 2001, and its Aftermath: Civil Liberties and the ,War on Terror". Workshop for high school English teachers in Baden-Württemberg „Responses to the Challenges to the USA After 9/11 and New Orleans”. Carl Schurz Haus. Freiburg i. Br., 25.02.2006.

\section{7}

Silverman, E.: „Strafrecht in den USA: Einführung und aktuelle Forschungsfragen zum plea bargaining". Vortrag im Rahmen der Summer School der Strafrechtlichen Abteilung des Max-Planck-Instituts für ausländisches und internationales Strafrecht zum Thema „Aktuelle Kriminalitätsentwicklung, Internationales Strafrecht und Strafrechtsvergleichung. Forschungsfragen an den Grenzen des Strafrechts“. Pécs/Ungarn, 19.06.2007.

\section{Son, Misuk}

\section{7}

Son, M.: „Substanz und Perspektive des koreanischen Strafrechts“. Seoul Sirip Universität. Seoul/Korea, 25.06.2007.

\section{Spoenle, Jan}

\section{7}

Spoenle, J.: „Technische und rechtliche Aspekte der Online-Durchsuchung“. Fachhochschule Offenburg. Offenburg, 29.11.2007.

Spoenle, J.: „Change Of The PhDnet Statute“. Max Planck PhDnet General Meeting 2007. Berlin, 09.11.2007.

\section{Tellenbach, Silvia}

\section{6}

Tellenbach, S.: „Islamic Law and the Inviolability of Bodily Integrity“. Workshop „Secular \& Religious Sources of Human Rights Law". Irmgard Coninx Foundation. Berlin, 19.05.2006.

Tellenbach, S.: „Islamisches Strafrecht heute“. Universität Jena. Jena, 12.06.2006.

Tellenbach, S.: „Freedom of Expression in Germany“ und „Study of Domestic Case-Law Germany“. Workshop „New Challenges and Restrictions on the Freedom of Expression". Bahçeşehir University. Istanbul/Türkei 16./17.11.2006.

Tellenbach, S.: „Zur Freiheit der Meinungsäußerung im Spiegel der neuen türkischen Strafgesetze“. Jahrestagung der Deutschen Arbeitsgemeinschaft Vorderer Orient (DAVO). Hamburg, 02.12.2006. 
2007

Tellenbach, S.: „Ehrenmorde an Frauen in der arabischen Welt und in der Türkei“. Bremer Gesellschaft. Freiburg i. Br., 23.01.2007.

Tellenbach, S.: „Zum Recht in der islamischen Republik Iran“. Tagung Iran und der „Kampf der Kulturen“. Katholische Akademie Trier. Trier, 31.01.2007 und 23.05.2007.

Tellenbach, S.: „Kurs zum türkischen Verfassungsrecht und Strafrecht". Bundesamt für Verfassungsschutz. Köln 01./02.03.2007.

Tellenbach, S.: „La loi allemande instituant un code de droit pénal international du 26 juin 2002“. Tagung Justice pénale: quelle évolution? Faculté des Sciences Juridiques, Économiques et de Gestion de Jendouba. Jendouba/Tabar$\mathrm{ka} /$ Tunesien, 10.03.2007.

Tellenbach, S.: „Free Speech and Freedom of Opin ion“. Tagung „Turkey in the Area of Freedom, Security and Justice - Legal and Political Aspects, The Research Center for International and European Law on Immigration and Asylum“. Universität Konstanz in Kooperation mit der Koç University. Istanbul/Türkei, 19.03.2007.

Tellenbach, S.: „Hakaret Suçları“ (Ehrverletzungsdelikte). Bahçeşehir Universität. Istanbul/Türkei, 20.03.2007.

Tellenbach, S.: „Zwischen religiösem und säkularem Recht in muslimischen Ländern“. Tagung Islam und Rechtsstaat - Zwischen Scharia und Säkularisierung. Kon rad-Adenauer-Stiftung e. V. und Zentrum Moderner Orient. Berlin, 06.09.2007.

Tellenbach, S.: „The Sharia in German Criminal Law?” The Sharia in Transnational Contexts: How does the Sharia Travel - Research on Islam in the Max Planck Society. Second Workshop. Freiburg i. Br., 26.09.2007.

Tellenbach, S.: „Il Codice Penale Turco“. Universität Verona/Universität Trento. Verona/Trento/Italien, 22./23.10.2007.

Tellenbach, S.: „Das neue türkische Strafgesetzbuch“. Universität Tiflis. Tiflis/Georgien, 26.10.2007.

Tellenbach, S.: „Der Ehrenmord - Streiflichter zum Strafrecht in Jordanien, der Türkei und Deutschland“. Universität Göttingen. Göttingen, 20.11.2007.

\section{Wade, Marianne}

2006

Wade, M.: „Development of the Public Prosecution institution: Case studies - Presentation and comparative study of the problems and obstacles". Vortrag auf der UNDP-POGARKonferenz für das „Programme on Strengthening the Rule of Law in the Arab States - Modernization of Public Prosecution Offices" unter der Schirmherrschaft des marokkanischen Justizministeriums. Rabat/Marokko, 24.02.2006.

Wade, M.: „The Rise of Prosecutorial Power in Europe“. Max-Planck-Institut für ausländisches und internationales Strafrecht. Freiburg i. Br., 14.08.2006

Wade, M.: „Fear v. Freedom post 9/11 - A European Perspective“. Vortrag im Rahmen der Panel-session „Fear versus Freedom post 9/11 - European Perspectives" bei der 6. Jahrestagung der European Society of Criminology. Tübingen, 28.08.2006.

Wade, M.: „Prosecutorial Case-ending - Faster, More Flexible, Fairer?" Vortrag im Rahmen der mit Prof. JörgMartin Jehle veranstalteten doppelten Panel-session „The Increasing Importance of Public Prosecution Services within Criminal Justice Systems" bei der 6. Jahrestagung der European Society of Criminology. Tübingen, 29.08.2006.

Wade, M.: „Trends in Prosecution“. Vortrag im Rahmen einer EUROJUST-Plenarsitzung. Den Haag/Niederlande, 19.09.2006
Wade, M.: „Videoüberwachung ist kein Allheilmittel“. Vortrag bei der Tagung „Videoüberwachung im öffentlichen Raum" des Berner Forums für Kriminalwissenschaften. Bern/Schweiz, 17.11.2006.

\section{7}

Wade, M.: „Deal or no deal - bestraft und trotzdem unschuldig?" Schulvortrag im Rahmen der Ersten Freiburger Schulvorträge an der Angell Akademie. Freiburg i.Br., 16.07.2007.

Wade, M.: „Criminal Justice in Europe“. Brasilianischdeutscher Workshop „Research on Crime, Criminal Law and Criminology“. Getulio Vargas Foundation und Max-PlanckInstitut für ausländisches und internationales Strafrecht in Zusammenarbeit mit dem Brazilian Center of Analysis and Planning. Harnack-Haus. Berlin, 24.07.2007.

Wade, M.: „Prosecutorial Control of Police Investigations“. Vortrag im Rahmen des Besuches der OSZE Spillover Mission to Skopje, Macedonia Delegation. Freiburg i. Br., 3.10.2007.

Wade, M.: „Prosecutorial Discretion and the Circumvention of Human Rights“. Vortrag im Rahmen des Workshops „Reforming Criminal Justice12: Key Issues of Contemporary Criminal Procedure“ und des Besuches einer Expertendelegation des Rule of Law Program der KonradAdenauer-Stiftung. Freiburg i. Br., 15.10.2007.

\section{Wahl, Thomas}

2006

Wahl, T.: „Data Protection under the Third Pillar". Summer Course on European Criminal Justice. ERA Congress Center. Trier, 06.07.2006.

Wahl, T.: „The Legal Framework for Data Protection in the EU and the New Data Retention Directive“. European Commission - TAIEX Seminar on Data protection under the Third Pillar: EU Information Systems and the Protection of Individual Rights. Tallinn/Estland, 26.10.2006.

Wahl, T.: „The Legal Framework for Data Protection in the EU“. European Commission - TAIEX Seminar on Data protection under the Third Pillar: EU Information Systems and the Protection of Individual Rights. Nicosia/Zypern; Valetta/Malta, 16./30.11.2006.

Wahl, T.: „The New Data Retention Directive 2006/24/ EC“. European Commission - TAIEX Seminar on Data protection under the Third Pillar: EU Information Systems and the Protection of Individual Rights. Valetta/Malta, 30.11.2006.

\section{7}

Wahl, T.: „The Legal Framework for Data Protection in the EU" und "The New Data Retention Directive 2006/24". European Commission - TAIEX Seminar on Data protection under the Third Pillar: EU Information Systems and the Protection of Individual Rights. Bukarest/Rumänien, 22.02.2007.

Wahl, T.: „Der Grundsatz der Verfügbarkeit - Revolution der internationalen Rechtshilfe in Strafsachen?" Doktorandenforum des Max-Planck-Instituts für ausländisches und internationales Strafrecht. Freiburg i. Br., 22.03.2007.

Wahl, T.: „Introduction to the European Court of Human Rights and the Case ,Ramanauskas v. Lithuania“. „Dialogue on Human Rights - Dialog zwischen Juristen aus Freiburg und Isfahan“. Kommission für Menschenrechte des Vereins der Richter und Staatsanwälte und des Anwaltvereins Freiburg. Freiburg i. Br., 27.03.2007.

Wahl, T.: „Constitutional limits to the binding effect of EC obligations in criminal matters - the situation in the 
German system“. Decennale del Centro di Diritto Penale Europeo. Convegno Internazionale, Per un rilancio del progetto europeo: esigenze di tutela degli interessi communitari e nuove strategie di integrazione penale in attesa della Costituzione per l'Europa. Facoltà di Giurisprudenza. Catania/ Italien, 25.05.2007.

Wahl, T.: „Europäisches Strafrecht: Einführung und aktuelle Forschungsfragen unter besonderer Berücksichtigung der Frage nach der Rechtssetzungskompetenz der EG im Strafrecht". Vortrag im Rahmen der Summer School der Strafrechtlichen Abteilung des Max-Planck-Instituts für ausländisches und internationales Strafrecht zum Thema „Aktuelle Kriminalitätsentwicklung, Internationales Strafrecht und Strafrechtsvergleichung. Forschungsfragen an den Grenzen des Strafrechts“. Pécs/Ungarn, 20.06.2007.

Wahl, T.: „Pillars‘ and ,Passerelle: The Legal Basis for the Third Pillar". Summer Course on European Criminal Justice. ERA Congress Center. Trier, 02.07.2007.

\section{Weigend, Ewa}

2007

Weigend, E.: „Der Europäische Haftbefehl und die polnische Verfassung". Universität Ljubljana. Ljubljana/Slowenien, 23.04.2007.

Weigend, E.: „Badania przesiewowe w niemieckim prawie karnym“ („Rasterfahndung im deutschen Strafrecht“). Jagiellonen Universität. Krakau/Polen, 29.05.2007.
Weigend, E.: „Orzeczenie niemieckiego Federalnego Trybunału Konstytucyjnego na temat badań przesiewowych“ („Rasterfahndung im Lichte der Entscheidung des deutschen Verfassungsgerichts“). Universität Warschau. Warschau/Polen, 31.05.2007.

\section{Wößner, Gunda}

2007

Wößner, G.: „Lucky Luke und der Psycho-Dok - oder: Soll man gefährliche Straftäter behandeln oder wegsperren?" Schulvorträge im Rahmen der Ersten Freiburger Schulvorträge an der Max-Weber- und der Richard-Fehrenbach-Schule sowie am Angell-Gymnasium. Freiburg i. Br. 04./16./17.07.2007.

Wößner, G.: „Werden wir den Insassen der Sozialtherapie gerecht, wenn wir Sexualstraftäter als ,besondere Klientel behandeln?" 11. Überregionale Fachtagung der Sozialtherapeutischen Einrichtungen im Justizvollzug. Halle/ Saale, 27.09.2007.

Wößner, G.: „Classifying Sexual Offenders - An Empirical Model for Generating Type-Specific Approaches to Intervention“. 7th Annual Conference of the European Society of Criminology. Bologna/Italien, 29.09.2007.

Wößner, G.: „Nutzen und Kosten der Sozialtherapie unter ökonomischen Gesichtspunkten: Was wissen wir, wofür evaluieren wir?" Expertenkolloquium Evaluation der Sozialtherapie im Justizvollzug. Wiesbaden, 14.11.2007. 


\section{Lehre}

\section{Albrecht, Hans-Jörg}

\section{Sommersemester 2006}

Vorlesung ,Jugendstrafrecht, Sanktionenrecht, Kriminologie II“, Albert-Ludwigs-Universität Freiburg i. Br.

Seminar „Neue und verdeckte Ermittlungsmethoden“, Albert-Ludwigs-Universität Freiburg i. Br.

\section{Wintersemester 2006/2007}

Vorlesung „Kriminologie I“, Albert-Ludwigs-Universität Freiburg i. Br.

Seminar „Immigration und Kriminalität“, Albert-Ludwigs-Universität Freiburg i. Br.

\section{Sommersemester 2007}

Vorlesung „Jugendstrafrecht“, Albert-Ludwigs-Universität Freiburg i. Br.

Seminar „Geldwäsche, Finanzierung des Terrorismus und Kontrolle", Albert-Ludwigs-Universität Freiburg i. Br.

\section{Wintersemester 2007/2008}

Vorlesung „Kriminologie I“, Albert-Ludwigs-Universität Freiburg i. Br.

Seminar „Kriminalitätsopfer, Opferschutz und Strafverfahren“, Albert-Ludwigs-Universität Freiburg i. Br.

\section{Arnold, Jörg}

Sommersemester 2006

Vorlesung und Übung „Deutsch-deutsche Strafrechtsgeschichte“ mit Examensklausuren, Westfälische WilhelmsUniversität Münster

\section{Sommersemester 2007}

(mit Theile, Hans) Examensseminar „Feindstrafrecht", Westfälische Wilhelms-Universität Münster.

\section{Brandenstein, Martin}

Wintersemester 2006/2007

(mit Özsöz, Figen) Seminar „Jugendkriminalität: Erscheinungsformen und Erklärungsansätze - unter besonderer Berücksichtigung fremdenfeindlich motivierter Straftaten“, Evangelische Fachhochschule Freiburg i. Br.

\section{Engelhart, Marc}

Wintersemester 2006/2007

Zusatzexamensklausurenkurs. Klausur Strafrecht (ab 6. Semester), Albert-Ludwigs-Universität Freiburg i. Br.

\section{Kilchling, Michael}

Wintersemester 2006/2007

Vorlesung „Strafvollzugsrecht“, Albert-Ludwigs-Universität Freiburg i. Br.

\section{Sommersemester 2007}

Vorlesung „Sanktionenrecht: Recht und Praxis strafrechtlicher Sanktionen und der Strafzumessung", AlbertLudwigs-Universität Freiburg i. Br.

\section{Wintersemester 2007/2008}

Vorlesung „Strafvollzugsrecht“, Albert-Ludwigs-Universität Freiburg i. Br.

\section{Köllisch, Tilman}

Wintersemester 2007/2008

Seminar „Soziologie abweichenden Verhaltens“, AlbertLudwigs-Universität Freiburg i. Br.

\section{Koch, Hans-Georg}

Sommersemester 2006

Vorlesung zu medizinrechtlichen Grundfragen im Rahmen des rechtsmedizinischen Teils des „Ökologischen Kurses" für MedizinstudentInnen, Albert-Ludwigs-Universität Freiburg i. Br.

Medizinrechtliches Seminar „Aktuelle Entwicklungen des Arztrechts“, Albert-Ludwigs-Universität Freiburg i. Br.

\section{Wintersemester 2006/2007}

Vorlesung „Einführung in das Medizinrecht“, AlbertLudwigs-Universität Freiburg i. Br.

\section{Sommersemester 2007}

Vorlesung zu medizinrechtlichen Grundfragen im Rahmen des rechtsmedizinischen Teils des „Ökologischen Kurses“ für MedizinstudentInnen, Albert-Ludwigs-Universität Freiburg i. Br.

\section{Wintersemester 2007/2008}

Seminar „Grundlagen des Medizinrechts“, Albert-Ludwigs-Universität Freiburg i. Br.

\section{Kouassi, Adome Blaise}

Wintersemester 2006/2007

Vorlesung „Zivilrecht (Handelsrecht)“, Universität Haute-Alsace, Colmar/Frankreich.

\section{Wintersemester 2007/2008}

Vorlesung „Zivilrecht (Handelsrecht)“, Universität Haute-Alsace, Colmar/Frankreich.

\section{Sommersemester 2007}

Vorlesung „Zivilrecht (Handelsrecht)“, Universität Haute-Alsace, Colmar/Frankreich. 


\section{Sieber, Ulrich}

Sommersemester 2006

Vorlesung „Informationsstrafrecht“, Albert-LudwigsUniversität Freiburg i. Br.

Vorlesung „Informationsrecht“, Ludwig-MaximiliansUniversität München.

\section{Wintersemester 2006/2007}

Interdisziplinäres Seminar „Phishing, Filesharing, WarDriving ... - aktuelle Fragen des Cybercrime“, Albert-Ludwigs-Universität Freiburg i. Br.

\section{Sommersemester 2007}

Vorlesung „Informationsstrafrecht“, Albert-LudwigsUniversität Freiburg i. Br.

Vorlesung „Informationsrecht“, Ludwig-MaximiliansUniversität München.

\section{Wintersemester 2007/2008}

Seminar Aktuelle Entwicklungen des Informationsstrafrechts“, Albert-Ludwigs-Universität Freiburg i. Br.

\section{Oberwittler, Dietrich}

Sommersemester 2007

Seminar „Sozialer Wandel und Kriminalität. Soziologische Erklärungen der Veränderungen von abweichendem Verhalten“, Universität Bielefeld.

\section{Oberwittler, Dietrich}

Wintersemester 2007/2008

(mit Blinkert, Baldo und Kaufmann, Stefan) Kolloquium „Sicherheit in modernen Gesellschaften“, Albert-Ludwigs-Universität Freiburg i. Br.

\section{Özsöz, Figen}

Wintersemester 2006/2007

(mit Brandenstein, Martin) Seminar ,Jugendkriminalität: Erscheinungsformen und Erklärungsansätze - unter besonderer Berücksichtigung fremdenfeindlich motivierter Straftaten“, Evangelische Fachhochschule Freiburg i. Br.

\section{Rinceanu, Johanna}

Wintersemester 2007/2008

Vorlesung „Einführung in das deutsche Strafprozessrecht für ausländische Studierende und Gastwissenschaftler“, Albert-Ludwigs-Universität Freiburg i. Br.

Vorlesung „Arbeits- und Bildungsrecht“, Evangelische Fachhochschule Freiburg i. Br.

\section{Wade, Marianne}

Wintersemester 2007/2008

(mit Pieth, Mark) Master-Programm „Straftaten gegen die Finanzinteressen der EU“, Universität Basel. 


\section{Veranstaltungen}

\section{VERANSTALTUNGEN AM INSTITUT}

\section{Tagungen und Kolloquien}

\section{6}

Kolloquium über European Criminal Justice/Criminology Study Tour, Mount Royal College, 03.03.2006.

Kolloquium mit einer Delegation von Richtern des Tribunal Supremo aus Madrid, 15.05.2006.

Kolloquium mit einer chinesischen Expertengruppe zum Strafvollzug, 06.06.2006.

Kolloquium zum 40-jährigen Bestehen des Max-PlanckInstituts für ausländisches und internationales Strafrecht mit einer interdisziplinären Diskussion über „Aufgaben, Methoden und Perspektiven der Rechtsvergleichung im Zivilrecht, Öffentlichen Recht, Strafrecht, Kriminologie, Arbeitsrecht und Prozessrecht", 21.07.2006.

Kolloquium mit einer US-amerikanische Besuchergruppe: Studenten der Central Missouri State University, 03./08.08.2006

Kolloquium mit einer Delegation des Goethe-Instituts, (EU-Sprachkurs 2006 für höhere Beamte aus den Justizministerien und von Obersten Gerichten der EU-Mitgliedsund -Beitrittsländer, höhere Bedienstete der EU-Kommission und des Rates der EU, des Europäischen Gerichtshofs und von EUROJUST), 16.10.2006.

\section{7}

Kolloquium mit einer Delegation von Polizeirechtsexperten aus der Türkei, 19.01.2007.

Internationaler Workshop „Überlegungen zu eine europäischen Strafrechtspflege“, 20./21.01.2007.

Interdisziplinäres Seminar zu aktuellen Fragen des Cybercrime, 26./27.01.2007.

Kolloquium mit einer weiteren Delegation von Polizeirechtsexperten aus der Türkei, 10.02.2007.

Internationaler Workshop „Familiale Tötungsdelikte mit anschließendem Suizid in Europa“, 08./09.03.2007.

Kolloquium mit Richtern und Mitarbeitern des ICC zu Legal Tools des ICC, 27.04.2007.

Kolloquium mit Prof. Benjamin B. Ferencz, USA: „Lessons of Nuremberg", 26.07.2007.

Kolloquium mit einer Gruppe von Studierenden der Universität Kumamoto, Japan, 17.09.2007.

Kolloquium mit einer Delegation der OSZE. Aus tausch mit Wissenschaftlerinnen und Wissenschaftlern zu Fragen der Reform des Justizsystems in Mazedonien, 02./03.10.2007.

Workshop in Zusammenarbeit mit dem Rechtsstaatsprogramm der Konrad-Adenauer-Stiftung über „Reforming Criminal Justice: Key Issues of Contemporary Criminal Procedure", 15.10.2007.

\section{Vorträge in der Strafrechtlichen Abteilung (insbesondere „Mittwochsvorträge“)}

\section{6}

Dr. Adome Blaise Kouassi, Max-Planck-Institut für ausländisches und internationales Strafrecht, Freiburg i.Br.: „Rechtssysteme in Afrika südlich der Sahara“, 01.02.2006.

Dr. Enara Garro Carrera, Spanien: ,Strafrechtliche Wiedergutmachung: Grundlagen ihrer Relevanz bei der Strafzumessung“, 08.03.2006.

Dr. Jan Nemitz/Steffen Wirth, ICTY,Den Haag, Niederlande: „Aktuelle Rechtsfragen und praktische Aspekte der Arbeit am Jugoslawien-Tribunal“, 12.05.2006.

Professor Dr. K.D. Gaur, Indien: „Human Rights Movement in India“, 12.06.2006.

Mustafa Abdel Baqi; Palästina: „Legal Reform in Period of Transition and State-Building: The Case of Palestine“, 28.06.2006.

Lesung Astrid v. Pufendorf: „Die Plancks - eine Familie zwischen Patriotismus und Widerstand“, 25.07.2006.

Dr. Valentina Caccamo, Italien: „Notwehr nach dem Römischen Recht“, 26.07.2006.

Prof. Roque de Brito Alves, Brasilien: „Constitution and Criminal Law“, 13.09.2006.

Paul Rabbat, Max-Planck-Institut für ausländisches und internationales Strafrecht, Freiburg i.Br.: „Judging Saddam Hussein: The Iraqi High Tribunal and the Dujail Case", 07.12.2006.

\section{7}

Taehoon Lee, Präsident des Korean Institute of Criminal Justice Policy Seoul/Korea: „The Arrest System in Korea“, 07.02.2007.

Dr. Catalina Benaventes, Spanien: „Einschränkungen von Rechten Terrorverdächtiger bei polizeilicher Festnahme in Spanien“, 14.03.2007.

Prof. Faraldo Cabana, Spanien: „Besonderheiten im Strafvollzug für Terroristen in Spanien seit 2003“, 14.03.2007.

Prof. Mark Findlay: „Global governance and criminal law", 16.05.2007

Dr. Patricia EsquinaValverde, Spanien: „Täter-OpferMediation im Bereich der häuslichen Gewalt an Frauen“, 23.05.2007.

Prof. Dr. Raymond Teske, USA: „Constitutional Issues Regarding Child Pornography in the United States", 06.06.2007.

Dr. Mia Swart, Südafrika: The Wouter Basson Prosecution of Apartheid Crimes in South Africa“, 11.07.2007.

Dr. Oren Gazahl, Israel: „Economic Analysis of Plea Bargaining", 18.07.2007. 
Jon Petter Rui Johansen, Norwegen: „Ne bis in idem“, 25.07.2007.

Prof. Mohsen Rahami, Iran: „Counterterrorism Rules in the Islamic Law and the Iranian Criminal Law", 08.08.2007.

Dr. Aleksandra Lysova, Russland: „Intimate Partner Violence in Russia“. (19.09.2007)

Prof. Dr. Dr. h.c.mult. Francisco Muñoz Conde, Spanien: „Der Kampf gegen den Terrorismus innerhalb des rechtsstaatlichen Strafrechts“, 16.10.2007.

Prof. Dr. Dr. h.c.mult. Günther Jakobs, Bonn: „An den Grenzen rechtlicher Orientierung: Feindstrafrecht", 24.10.2007.

Inger Marie Sunde, Norwegen: „Internet Crime and International Re-Use of Legal Decisions“, 31.10.2007.

Judge Carmel A. Agius, ICTY, Den Haag/Prof. Dr. Dr. h.c. mult. Albin Eser, Freiburg: „Challenges of International Criminal Justice: Lessons from The Hague“, 13.11.2007.

Mohammed Wattad, Israel: „The Rome Statute \& Captain Planet: What lies between ,Crimes against Humanity' and the ,Natural Environment", 21.11.2007.

Prof. Eric Luna, USA: „Law Enforcement and Discretion in the USA“, 28.11.2007.

Dr. Shawn Boyne, USA: „Principle of Legality - Practical Limitations“, 28.1 1.2007.

\section{Vorträge in der Kriminologischen Abteilung}

\section{6}

Prof. Per-Olof H. Wikström, Institute of Criminology, University of Cambridge; United Kingdom: „A Theory of Moral Action“, 04.04.2006.

Prof. Carlos Elbert, Buenos Aires, Argentinien: „Auf der Suche nach einer neuen Kriminalpolitik - aber welcher?" 22.05.2006.

Prof. Mohammad Frajiha in collaboration with Prof. Firouz Mahmoudi and Dr. Hassan Rezaei: „Main Aspects of Sanction and Sentencing System in Post Revolutionary Iran“, 20.09.2006

Dr. Eugene M. Hyman, Hon. Judge am Superior Court of California, County of Santa Clara: „Domestic and Family Violence Courts: the role of specialized courts in responding to social problems“, 20.11.2006
2007

Prof. Jost Reinecke, Universität Bielefeld: „Klassifikation von Delinquenzverläufen. Eine Anwendung von Mischverteilungsmodellen", 16.05.2007.

Prof. Gao Mingxuan, College for Criminal Law Sciences, Beijing University, VR China: „Development of Criminal Legislations in China“, 11.06.2007.

Prof. Mohsen Rahami, University of Tehran, Iran: „Counterterrorism Rules in the Islamic Law and the Iranian Criminal Law", 08.08.2007.

Prof. David S. Wall, University of Leeds, United Kingdom: „The challenge of cybercrime for criminal justice“, 11.12.2007.

\section{Vorträge während der Doktorandentreffen}

2006

Malaika Nolde: „Inpflichtnahme Privater im Rahmen der Strafverfolgungsvorsorge“, 23.02.2006

Holger Rohne: „Opferperspektiven im interkulturellen Vergleich - Eine viktimologische Studie im Kontext der Alqsa-Intifada“, 30.03.2006.

Nandor Knust: „Strafrecht und Gacaca“, 30.03.2006.

Frank M. Höfinger: „Hyperlinks und die Strafbarkeit für fremde Inhalte in Online-Medien“, 28.04.2006.

Phillip Brunst: „Anonymität im Internet“, 30.05.2006.

René M. Kieselmann: „Erstellung von Bewegungsprofilen im Strafverfahren“, 29.06.2006.

Marcello Bellini: „Hacken leicht gemacht!“. (26.10.2006)

Carolin Quenzer: „Sexualdelinquenz - Rückfälligkeit Prognose“, 23.11.2006.

\section{7}

Thomas Wahl: „Der Grundsatz der Verfügbarkeit - Revolution der Internationalen Rechtshilfe in Strafsachen?" 22.03.2007

Bianca Lafrenz: „Tötungsdelikte in der Familie - Erklärungsversuche und Risikofaktoren“, 03.05.2007.

Sonja Brauner: „Prognose bei Sexualstraftätern“, 12.07.2007.

Carolin Quenzer: „Anforderungen an Prognosegutachten“, 30.11.2007.

\section{EXTERNE VERANSTALTUNGEN DES INSTITUTS}

\section{6}

Tagungsreihe „Les Chemins de l'harmonisation penale“, Basel, Schweiz, 07.-10.06.2006.

\section{7}

Tagungsreihe „Les Chemins de l'harmonisation pénale“, Neapel, Italien, 11.-14.04.2007.

Brasilianisch-deutscher Workshop „Research on Crime, Criminal Law and Criminology“. Getulio Vargas Foundation und Max-Planck-Institut für ausländisches und internationales Strafrecht in Zusammenarbeit mit dem Brazilian Center of Analysis and Planning. Harnack-Haus. Berlin, 24.07.2007.

Deutsch-Argentinisches Symposium, „Die Gegenwart der Kriminalität, der Strafrechtsentwicklung und Strafrechtskritik“, anlässlich des Bestehens der 150-jährigen Beziehungen zwischen Deutschland und Argentinien, Buenos Aires, Argentinien, 13.-15.12.2007. 


\section{E. Doktoranden}

Doktoranden sind am Institut in unterschiedlicher Weise angebunden. Im Mittelpunkt der Doktorandenausbildung steht - vor allem in der Zukunft - die Ausbildung in den beiden Research Schools durch die Institutsdirektoren und die Professoren der Partneruniversitäten (vgl. oben Kapitel III). Die Direktoren betreuen am Institut im Rahmen ihrer Forschungsprogramme auch zahlreiche Doktoranden außerhalb der Research Schools. Hinzu kommen Dissertationen von Mitarbeitern und Gästen, die bei externen Professoren an anderen Universitäten promovieren. Auch betreuen wissenschaftliche Mitarbeiter des Max-Planck-Instituts Doktoranden außerhalb ihrer Institutstätigkeit an anderen Universitäten. Die nachfolgende Aufstellung nennt die Doktoranden, die in den beiden Forschungsabteilungen des Instituts im Rahmen der Max-Planck-Forschung in ihrem Dissertationsverfahren betreut wurden.

\section{STRAFRECHTLICHE ABTEILUNG ${ }^{1}$}

\begin{tabular}{|c|c|c|}
\hline Name & Thema & Betreuer \\
\hline Dingler, Andreas & Betrug bei Online-Auktionen & $\begin{array}{l}\text { Prof. Dr. Ulrich Sieber } \\
\text { (Rigorosum: 15.2.2008) }\end{array}$ \\
\hline Engelhart, Marc & $\begin{array}{l}\text { Verantwortlichkeit und Sanktionierung von Unternehmen } \\
\text { unter besonderer Berücksichtigung von Compliance- } \\
\text { Maßnahmen }\end{array}$ & Prof. Dr. Ulrich Sieber \\
\hline Forster, Susanne & $\begin{array}{l}\text { Eingriffe in die Bewegungsfreiheit durch die Terroris- } \\
\text { musgesetzgebung des Vereinigten Königreichs }\end{array}$ & Prof. Dr. Ulrich Sieber \\
\hline $\begin{array}{l}\text { Höfinger, } \\
\text { Frank-Michael }\end{array}$ & $\begin{array}{l}\text { Hyperlinks und die Strafbarkeit für fremde Inhalte der } \\
\text { Online-Medien }\end{array}$ & Prof. Dr. Ulrich Sieber \\
\hline Jarvers, Konstanze & $\begin{array}{l}\text { Neue Wege zur Behandlung der Massen- und Kleinkrimi- } \\
\text { nalität in Italien: Alternative Erledigungsmöglichkeiten } \\
\text { und (neue) Sanktionsformen im Verfahren vor dem } \\
\text { Friedensrichter }\end{array}$ & $\begin{array}{l}\text { Prof. Dr. Ulrich } \\
\text { (Rigorosum: 12.2.2007) }\end{array}$ \\
\hline Kieselmann, René & $\begin{array}{l}\text { Lokalisation von Verdächtigen durch Ortung von Mobil- } \\
\text { telefonen }\end{array}$ & Prof. Dr. Ulrich Sieber \\
\hline Knust, Nandor & $\begin{array}{l}\text { Strafrecht und Gacaca - Die Aufarbeitung des ruan- } \\
\text { dischen Völkermords mit einem pluralistischen Rechts- } \\
\text { modell }\end{array}$ & Prof. Dr. Ulrich Sieber \\
\hline Macke, Julia & $\begin{array}{l}\text { Internationalisierung von Strafrecht durch den Sicher- } \\
\text { heitsrat der Vereinten Nationen }\end{array}$ & Prof. Dr. Ulrich Sieber \\
\hline Maljević, Almir & $\begin{array}{l}\text { Mitgliedschaft in einer kriminellen „Vereinigung” und } \\
\text { "Conspiracy“ als Modelle zur Strafverfolgung von organi- } \\
\text { sierter Kriminalität }\end{array}$ & Prof. Dr. Ulrich Sieber \\
\hline
\end{tabular}

Alle Dissertationen sind in das Forschungsprogramm der strafrechtlichen Abteilung integriert und in der oben I.B.2 abgedruckten Projekttabelle erläutert oder den Projekten zugeordnet. Die dort nicht separat aufgeführten und im Folgenden genannten Dissertationen von Julie Trappe und Leonie von Braun sind Teilprojekte der Untersuchung "Strafrecht in Reaktion auf Systemunrecht", die Dissertation von Carola Seith ist Teil des Projekts "Der Status des extrakorporalen Embryos" 


\begin{tabular}{|c|c|c|}
\hline Müller, Tim & $\begin{array}{l}\text { Präventiver Freiheitsentzug als Instrument der } \\
\text { Terrorismusbekämpfung }\end{array}$ & Prof. Dr. Ulrich Sieber \\
\hline Nolde, Malaika & $\begin{array}{l}\text { Inpflichtnahme Privater im Rahmen der Strafverfolgungs- } \\
\text { vorsorge }\end{array}$ & Prof. Dr. Ulrich Sieber \\
\hline Pfützner, Peggy & $\begin{array}{l}\text { Das neue Strafverfahren zur Bekämpfung der } \\
\text { Organisierten Kriminalität in Frankreich }\end{array}$ & $\begin{array}{l}\text { Prof. Dr. Ulrich Sieber } \\
\text { (Rigorosum: 10.7.2007) }\end{array}$ \\
\hline Rabbat, Paul & $\begin{array}{l}\text { Towards an Offence of Terrorism at International } \\
\text { Criminal Law? }\end{array}$ & Prof. Dr. Ulrich Sieber \\
\hline Rinceanu, Johanna & Völkerstrafrecht in Rumänien & $\begin{array}{l}\text { Prof. Dr. Jörg Arnold } \\
\text { (Disputation: 16.2.2007) }\end{array}$ \\
\hline Seith, Carola & Status und Schutz des extrakorporalen Embryos & $\begin{array}{l}\text { Prof. Dr. Albin Eser } \\
\text { (Rigorosum: 29.11.2006) }\end{array}$ \\
\hline Sonderegger, Linus & Grenzen des Folterverbots & Prof. Dr. Ulrich Sieber \\
\hline Spoenle, Jan & Probleme der Strafverfolgung im Bereich des Cybercrime & Prof. Dr. Ulrich Sieber \\
\hline Trappe, Julie & $\begin{array}{l}\text { Strafrechtliche Aufarbeitung des Systemunrechts } \\
\text { in Rumänien nach } 1989\end{array}$ & $\begin{array}{l}\text { Prof. Dr. Jörg Arnold } \\
\text { (Rigorosum: 16.6.2008) }\end{array}$ \\
\hline von Braun, Leonie & $\begin{array}{l}\text { Internationalisierte Strafgerichte. Eine Analyse der } \\
\text { Strafverfolgung schwerer Menschenrechtsverletzungen } \\
\text { in Osttimor, }\end{array}$ & $\begin{array}{l}\text { Prof. Dr. Jörg Arnold } \\
\text { (Rigorosum: 5.7.2007) }\end{array}$ \\
\hline Zhou, Zunyou & $\begin{array}{l}\text { Terrorismusbekämpfung im Spannungsfeld von Sicherheit } \\
\text { und Freiheit in Deutschland und China }\end{array}$ & Prof. Dr. Ulrich Sieber \\
\hline
\end{tabular}

\section{KRIMINOLOGISCHE ABTEILUNG}

\begin{tabular}{|c|c|c|}
\hline Name & Thema & Betreuer \\
\hline $\begin{array}{l}\text { Abdelbaqi, Mustafa } \\
\text { Hussein }\end{array}$ & $\begin{array}{l}\text { The Administration of Criminal Justice in Palestine - } \\
\text { Development, Reform and Challenges }\end{array}$ & Prof. Dr. Hans-Jörg Albrecht \\
\hline Brandenstein, Martin & $\begin{array}{l}\text { Der Strafvollzug zwischen Übelzuführung und Resozia- } \\
\text { lisierung - eine empirische Studie }\end{array}$ & Prof. Dr. Helmut Kury \\
\hline $\begin{array}{l}\text { Briceño Peñlaver, } \\
\text { Gerardo José }\end{array}$ & $\begin{array}{l}\text { Prävention von Gewalt - rechtspolitische und kriminolo- } \\
\text { gische Ansätze im Umgang mit Gewalt }\end{array}$ & Prof. Dr. Hans-Jörg Albrecht \\
\hline Chao, Yen-Ching & $\begin{array}{l}\text { Einwirkungen der Grundrechte auf die Beweisverbote } \\
\text { im Strafprozessrecht - im Hinblick auf die Situation in } \\
\text { Taiwan und in der Volksrepublik China }\end{array}$ & $\begin{array}{l}\text { Prof. Dr. Hans-Jörg Albrecht } \\
\text { (Rigorosum: 12.2.2007) }\end{array}$ \\
\hline Ciftçi, Isa & $\begin{array}{l}\text { DNA im kriminalistischen und gesellschaftlichen } \\
\text { Kontext }\end{array}$ & Prof. Dr. Hans-Jörg Albrecht \\
\hline Fan, Wen & $\begin{array}{l}\text { Strafzumessung - Wirksamkeit des Strafvollzugs } \\
\text { und der Kriminalpolitik }\end{array}$ & Prof. Dr. Hans-Jörg Albrecht \\
\hline Gauthier, Jérémie & $\begin{array}{l}\text { La pertinance du critère ethnique dans l'étude du travail } \\
\text { policier. Une comparaison Franco-Allemande }\end{array}$ & Prof. Dr. Hans-Jörg Albrecht \\
\hline
\end{tabular}




\begin{tabular}{|c|c|c|}
\hline Getos, Anna Maria & Terrorismus und Hasskriminalität & Prof. Dr. Hans-Jörg Albrecht \\
\hline Ghassemi, Ghassem & $\begin{array}{l}\text { A Sociological Perspective of Iran's Penal Policy after } \\
\text { the Revolution of } 1979\end{array}$ & Prof. Dr. Hans-Jörg Albrecht \\
\hline Glet, Alke & Sozialkonstruktion und Verfolgung von Hasskriminalität & Prof. Dr. Hans-Jörg Albrecht \\
\hline Grafe, Adina & $\begin{array}{l}\text { Die Auskunftserteilung über Verkehrsdaten nach } \\
\S \S 100 \mathrm{~g}, 100 \mathrm{~h} \text { StPO - Staatliche Kontrolle unter Mitwir- } \\
\text { kung Privater }\end{array}$ & Prof. Dr. Hans-Jörg Albrecht \\
\hline Jessen, Daniela & $\begin{array}{l}\text { Die Implementation der Fußfessel in Hessen - } \\
\text { Eine Evaluation des Einführungsprozesses anhand } \\
\text { empirischer Analysen der Rechtswirklichkeit und } \\
\text { Effizienz des elektronisch überwachten Hausarrestes }\end{array}$ & Prof. Dr. Hans-Jörg Albrecht \\
\hline Kafatou, Maria & $\begin{array}{l}\text { The Media-Crime Nexus revisited. On the re-con- } \\
\text { struction of crime and law-and-order In Crime-appeal } \\
\text { programming }\end{array}$ & $\begin{array}{l}\text { Prof. Dr. Hans-Jörg Albrecht } \\
\text { (Rigorosum: 15.5.2006) }\end{array}$ \\
\hline $\begin{array}{l}\text { Khurelbaatar, } \\
\text { Erdem-Undrakh }\end{array}$ & $\begin{array}{l}\text { Das Sanktionensystem im mongolischen Strafrecht } \\
\text { im Vergleich mit dem deutschen Strafrecht }\end{array}$ & Prof. Dr. Hans-Jörg Albrecht \\
\hline Kunz, Franziska & $\begin{array}{l}\text { Infringement of Formal and Informal Norms by the } \\
\text { Elderly - Looking Behind the Curtain }\end{array}$ & Prof. Dr. Hans-Jörg Albrecht \\
\hline Kurzberg, Benjamin & $\begin{array}{l}\text { Jugendstrafe aufgrund schwerer Kriminalität - eine } \\
\text { Untersuchung zur Strafzumessung bei Jugendlichen, } \\
\text { Heranwachsenden und jungen Erwachsenen im Hinblick } \\
\text { auf den Erziehungsgedanken im Jugendstrafrecht }\end{array}$ & $\begin{array}{l}\text { Prof. Dr. Hans-Jörg Albrecht } \\
\text { (Rigorosum: 19.5.2008) }\end{array}$ \\
\hline Lafrenz, Bianca & $\begin{array}{l}\text { Tötungsdelikte in der Familie mit anschließendem Suizid } \\
\text { oder Suizidversuch - eine psychologische Betrachtung } \\
\text { dieses Phänomens in Deutschland }\end{array}$ & $\begin{array}{l}\text { Prof. Dr. Peter Streck } \\
\text { (Konstanz) }\end{array}$ \\
\hline Latsiou, Charikleia & $\begin{array}{l}\text { Präimplantationsdiagnostik - Rechtsvergleichung und } \\
\text { bioethische Fragestellungen. Eine Diskussion ange- } \\
\text { sichts der neuen Fortpflanzungsmedizin }\end{array}$ & $\begin{array}{l}\text { Prof. Dr. Hans-Jörg Albrecht } \\
\text { (Rigorosum: 11.7.2006) }\end{array}$ \\
\hline $\begin{array}{l}\text { Laule, } \\
\text { Juliane Karoline }\end{array}$ & $\begin{array}{l}\text { Berücksichtigung von Angehörigen bei der Auswahl und } \\
\text { Vollstreckung von Sanktionen }\end{array}$ & $\begin{array}{l}\text { Prof. Dr. Hans-Jörg Albrecht } \\
\text { (Rigorosum: 19.5.2008) }\end{array}$ \\
\hline Leibold, Tanja & $\begin{array}{l}\text { Der Deal im Steuerstrafrecht - Formen der Verständi- } \\
\text { gung im formellen und materiellen Recht }\end{array}$ & Prof. Dr. Hans-Jörg Albrecht \\
\hline Lien, Meng-Chi & $\begin{array}{l}\text { Entwicklungstendenzen des Opportunitätsprinzips im } \\
\text { Vergleich Taiwan, China, Deutschland }\end{array}$ & Prof. Dr. Hans-Jörg Albrecht \\
\hline Lukas, Tim & $\begin{array}{l}\text { Kriminalprävention in Großwohnsiedlungen - eine Eva- } \\
\text { luation baulicher und sozialer Maßnahmen in ost- und } \\
\text { westeuropäischen Hochhausquartieren }\end{array}$ & Prof. Dr. Hans-Jörg Albrecht \\
\hline Murphy, Chris & Geldwäsche und Glücksspiele & Prof. Dr. Hans-Jörg Albrecht \\
\hline $\begin{array}{l}\text { Nikolova Stamatova, } \\
\text { Nina }\end{array}$ & Organisierte Kriminalität in Bulgarien und Deutschland & Prof. Dr. Hans-Jörg Albrecht \\
\hline Olt, Gunther & $\begin{array}{l}\text { Pressefreiheit im Kontext strafprozessualer Ermittlungs- } \\
\text { maßnahmen }\end{array}$ & Prof. Dr. Hans-Jörg Albrecht \\
\hline Özsöz, Figen & $\begin{array}{l}\text { Rechtsextremismus und Jugendstrafvollzug - Aus- } \\
\text { wirkungen von Jugendhaft auf rechtsextremistische } \\
\text { Orientierungsmuster jugendlicher Gewalttäter }\end{array}$ & Prof. Dr. Helmut Kury \\
\hline
\end{tabular}




\begin{tabular}{|c|c|c|}
\hline Pathe, Imme & $\begin{array}{l}\text { Das System der verbotenen Internet-Angebote nach dem } \\
\text { JMStV }\end{array}$ & Prof. Dr. Hans-Jörg Albrecht \\
\hline Pehl, Dirk & $\begin{array}{l}\text { Die Implementation der Rasterfahndung - Eine empi- } \\
\text { rische Untersuchung zur Wirkmächtigkeit der gesetz- } \\
\text { lichen Regelungen zur operativen Informationserhebung } \\
\text { durch Rasterfahndung }\end{array}$ & $\begin{array}{l}\text { Prof. Dr. Hans-Jörg Albrecht } \\
\text { (Rigorosum: 19.5.2008) }\end{array}$ \\
\hline Qi, Shenghui & $\begin{array}{l}\text { Todesstrafe und öffentliche Meinung - unter besonderer } \\
\text { Berücksichtigung der Todesstrafe in China }\end{array}$ & Prof. Dr. Hans-Jörg Albrecht \\
\hline Quenzer, Carolin & $\begin{array}{l}\text { Jugendliche und Heranwachsende Sexualstraftäter } \\
\text { - eine empirische Studie über Rückfälligkeit und } \\
\text { Risikofaktoren }\end{array}$ & $\begin{array}{l}\text { PD Dr. Klaus-Peter Dahle } \\
\text { (Berlin) }\end{array}$ \\
\hline Rehaag, Konstantin & $\begin{array}{l}\text { Prinzipien von Täterschaft und Teilnahme im englischen } \\
\text { und deutschen Strafrecht }\end{array}$ & $\begin{array}{l}\text { Prof. Dr. Hans-Jörg Albrecht } \\
\text { (Rigorosum: 12.2.2007) }\end{array}$ \\
\hline $\begin{array}{l}\text { Rohne, } \\
\text { Holger-Christoph }\end{array}$ & Opferperspektiven im interkulturellen Vergleich & $\begin{array}{l}\text { Prof. Dr. Hans-Jörg Albrecht } \\
\text { (Rigorosum: 9.7.2007) }\end{array}$ \\
\hline Saux, María Soledad & $\begin{array}{l}\text { Terrorismusbekämpfung in der EU: Auswirkungen auf } \\
\text { das Asyl- und Einwanderungsrecht (am Beispiel Spani- } \\
\text { ens und Deutschlands) }\end{array}$ & Prof. Dr. Hans-Jörg Albrecht \\
\hline Sieler, Reinhard & $\begin{array}{l}\text { Die Effizienz der durch das „Gesetz zur Bekämpfung } \\
\text { des illegalen Rauschgifthandels und anderer Erschei- } \\
\text { nungsformen der Organisierten Kriminalität“" geregelten } \\
\text { polizeilichen Ermittlungsmethoden bei der Bekämpfung } \\
\text { der organisierten Kriminalität - eine Literaturanalyse }\end{array}$ & $\begin{array}{l}\text { Prof. Dr. Hans-Jörg Albrecht } \\
\text { (Rigorosum: 12.2.2007) }\end{array}$ \\
\hline Tavcer, Scharie & $\begin{array}{l}\text { Causal Factors in the Crime of Trafficking of Women for } \\
\text { the Purpose of Sexual Exploitation: An exploration into } \\
\text { push and pull factors relevant to women trafficked from } \\
\text { Moldova to Western Europe }\end{array}$ & $\begin{array}{l}\text { Prof. Dr. Hans-Jörg Albrecht } \\
\text { (Rigorosum: 6.8.2007) }\end{array}$ \\
\hline Tetal, Carina Renate & $\begin{array}{l}\text { Analyse von Deliktsähnlichkeiten auf der Basis von } \\
\text { Individualdaten der Freiburger Kohortenstudie }\end{array}$ & $\begin{array}{l}\text { Prof. Dr. Hans-Jörg Albrecht } \\
\text { (Rigorosum: 3.1.2008) }\end{array}$ \\
\hline Voß, Almuth & Sozialethische Einschränkungen des Notwehrrechts & Prof. Dr. Hans-Jörg Albrecht \\
\hline Walter, Britta & $\begin{array}{l}\text { Arbeitseinstellungen von Jugendrichtern und Jugend- } \\
\text { staatsanwälten in Deutschland }\end{array}$ & Prof. Dr. Hans-Jörg Albrecht \\
\hline Wang, Ying & $\begin{array}{l}\text { Strafrechtlicher Schutz des geistigen Eigentums: eine } \\
\text { vergleichende Untersuchung zum chinesischen und } \\
\text { deutschen Strafrecht }\end{array}$ & Prof. Dr. Hans-Jörg Albrecht \\
\hline Wildfang, Anne & $\begin{array}{l}\text { Terrorismus - eine kriminologische Studie vor dem } \\
\text { Hintergrund der Internationalisierung }\end{array}$ & Prof. Dr. Hans-Jörg Albrecht \\
\hline Winterer, Heide & $\begin{array}{l}\text { Strafverfolgung bei Gewalt im sozialen Nahbereich - } \\
\text { Entwicklungen und Tendenzen am Beispiel der Staats- } \\
\text { anwaltschaft Freiburg }\end{array}$ & Prof. Dr. Hans-Jörg Albrecht \\
\hline Wolf, Florian & $\begin{array}{l}\text { Die strafrechtliche Verantwortlichkeit des Arztes für die } \\
\text { Handlungen seiner untergebrachten psychiatrischen } \\
\text { Patienten }\end{array}$ & $\begin{array}{l}\text { Prof. Dr. Hans-Jörg Albrecht } \\
\text { (Rigorosum: 12.2.2007) }\end{array}$ \\
\hline
\end{tabular}




\section{IMPRESSUM}

\section{Herausgeber}

Max-Planck-Gesellschaft zur

Förderung der Wissenschaften e.V.

c/o Max-Planck-Institut für ausländisches

und internationales Strafrecht

Geschäftsführender Direktor 2007/2008

Prof. Dr. Dr. h.c. Ulrich Sieber

Günterstalstraße 73

D-79100 Freiburg

Tel.: +49 (0)761-7081-1

Fax: +49 (0)761-7081-294

E-Mail: info@mpicc.de

Internet: http://www.mpicc.de

\section{Druck}

Stückle Druck und Verlag

Stückle-Straße 1

77955 Ettenheim

\section{Satz}

Ines Hofmann

\section{Bildnachweise}

S. 5, 102: Martin Langhorst; andere Bilder soweit nicht anders angegeben: Max-Planck-Institut für ausländisches und internationales Strafrecht

\section{Gestaltung}

Layout und Titelbild:

Justmedia Design, Köln

Alle Rechte vorbehalten. (C) 2008 Max-Planck-Institut für ausländisches und internationales Strafrecht, Freiburg i. Br. 